Design: Thirty-six radical prostatectomy cases with a diagnosis of prostatic adenocarcinoma were chosen from our archival specimens, including 18 patients who developed recurrent cancer after curative surgery, and 18 patients whose cancers did not recur during matched follow up times. NED was evaluated by performing immunohistochemistry (IHC) for Chromogranin $\mathrm{A}(\mathrm{CgA}) .10$ cancer areas are randomly selected on each whole mount section, and the $\mathrm{CgA}$ IHC staining intensity in these areas was graded as $0-5$.

Results: Significant intra-tumoral heterogeneity of $\mathrm{CgA}$ staining intensity was observed, as illustrated in Figure 1A. The cumulative $\mathrm{CgA}$ scores from 10 areas were higher in specimens from patients whose cancers relapsed, as compared with specimens from patients whose cancers did not recur. Mean cumulative $\mathrm{CgA}$ score is $18.72 \pm 2.78$ in the relapsed group and $8.28 \pm 1.44$ in the remission group. (Figure 1B and C)
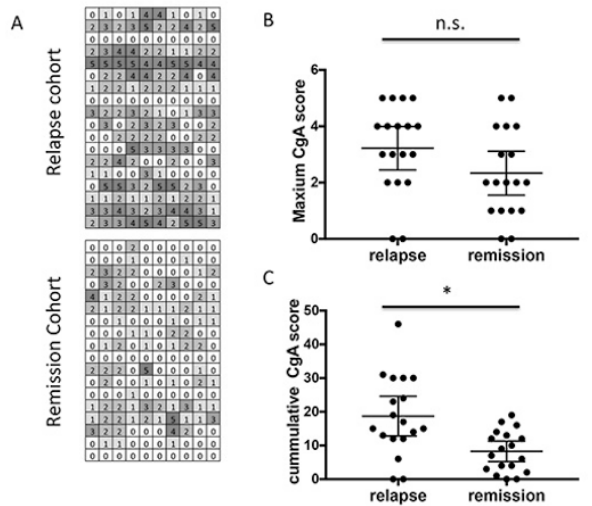

Figure 1: A) CPA scores of all specimens are illustrated as each row represents one specimen and each column represents one randomly selected area. B) Highest scores in each samples are plotted. C) Cumulative scores from 10 random areas are plotted. ${ }^{*} \mathrm{p}<0.05$

Conclusions: This study reveals that intra-tumor heterogeneity of NED exists in prostate adenocarcinoma and influences the precise evaluation of NED in clinical samples. Though the data is not conclusive, we do observe a lower level of NED in patients with remission compared to patients with relapsed cancer by thorough evaluation of NED in prostate whole mount sections. This study potentially provides guidance to clinical usage of NED in prostate adenocarcinoma.

\section{Gynecologic and Obstetric Pathology}

\section{Ovarian Granulosa Cell Tumors: A Surveillance, Epidemiology and End Results (SEER) Data Review of Prognostic Clinicopathological} Parameters in 1815 Patients

Eman Abdulfatah, Marcel T Ghanim, Oumaima Chaib, MHD Fayez Daaboul, Baraa Alosh, Khaleel I Al-Obaidy, Zaid Mahdi, Kinda Hayek, Sharif Sakr, Adnan R Munkarah, Sudeshna Bandyopadhyay, Rouba Ali-Fehmi. Wayne State University School of Medicine, Detroit, MI; Karmanos Cancer Center/Wayne State University, Detroit, MI; Henry Ford Health System, Detroit, MI.

Background: Ovarian granulosa cell tumors (GCTs) represent approximately 2-5\% of all ovarian cancers. The prognostic indicators of GCT are still controversial. The aim of this study is to identify the clinico-pathological variables affecting prognosis and survival outcome in patients with GCT.

Design: SEER database was searched for patients diagnosed with Adult (AGCT) and Juvenile (JGCT) Granulosa Cell Tumors. Demographic characteristics and prognostic factors including age, race, marital status, FIGO stage, surgery, tumor size and lymphadenectomy were analyzed using Log-Rank, Cox regression and Kaplan-Meier survival analyses.

Results: 1794 and 21 patients were identified with AGCT and JGCT, respectively. Of all the AGCT patients, $1081(60.2 \%)$ were surgically-unstaged while $395(22 \%), 80(4.5 \%)$, $158(8.8 \%)$ and $80(4.5 \%)$ were stages I, II, III and IV, respectively. Patients with AGCT $>50$ years of age had better 10 -year OS ( $85.3 \%$ vs. $63.8 \%, \mathrm{P}<0.05)$ (HR2.4 ,P:0.003), whereas patients with JGCT had a 3-year OS of $63.4 \%$. Surgically-staged I-II patients had better survival compared to non-staged and those with advanced stage III-IV (HR $10.6, \mathrm{P}=0.0005$ ) (10-year OS $93.8 \%, 69.9 \%, 49.4 \%$ respectively; $\mathrm{P}<0.05$ ). No survival difference was noted between stage IA,IB and stage IC nor between Caucasians and African Americans. Patients with tumors greater than $5 \mathrm{~cm}$ had significantly worse 10 year OS ( $98.1 \%$ vs $85.1 \%, \mathrm{P}<0.05)$. Lymphadenectomy in AGCT was associated with significantly better OS $(89.8 \%$ vs. $71.2 \%, \mathrm{P}<0.05)$. Cox regression analysis revealed that older age ( $>50$ years), early stage and lymphadenectomy were independently associated with better OS (HR 0.41, 0.01, and $0.49 ; \mathrm{P}<0.05$ respectively). Clinically stage I (surgically-unstaged) patients had worse 10-year OS compared to those with surgical stage I ( 70.3 vs $89.3 \%, \mathrm{P}<0.05)$.

Conclusions: Age, stage and lymphadenectomy were independent predictors of survival outcome in patients with ovarian granulosa cell tumor.
1093 Mucinous Differentiation Is Predictive of Improved Outcomes in Low Grade Endometrioid Carcinoma

Eman Abdulfatah, Sudeshna Bandyopadhyay, Oumaima Chaib, Andres A Roma, Denise Barbuto, Elizabeth Euscher, Bojana Djordjevic, Elizabeth E Frauenhoffer, Delia P Montiel, Anais Malpica, Elvio Silva, Rouba Ali-Fehmi. WSU, Detroit, MI; UC, San Diego, CA; CSMC, LA, CA; MDAnderson, Houston, TX; Ottawa, Ott, ON, Canada; Penn State, Hershey, PA; I N C, Mexico, Mexico.

Background: Endometrioid carcinoma(EC) is often associated with squamous or mucinous (MUC)differentiation(diff). The significance of MUC-diff in EC is yet to be determined. The aim of this study was to correlate the presence of MUC-diff with clinicopathologic features of a large multi-institutional cohort of low grade EC(LGEC) and its impact on clinical outcome.

Design: Retrospective review of LGEC(G1or2)(n=593) from 1991-2011 at 9 institution was conducted to evaluate clinicopathologic parameters:age, tumor size, stage,depth and pattern of myometrial invasion(MI),necrosis,papillary architecture,squamous and MUC-diff,lymphovascular invasion(LVI),lymph node involvement and outcomes. MUC-diff was defined as $\geq 10 \%$ cells with intracellular mucin. Data was analyzed. Results: Pts' median age was 61(22-91)yrs. MUC-diff was identified in 38\%. Although pts with MUC-diff were significantly older $(>60 \mathrm{yrs})$ and their tumors showed more papillary architecture and MELF pattern of MI with lack of necrosis $(\mathrm{P}=0.001, \mathrm{P}=0.00$ $1, \mathrm{P}=0.020, \mathrm{P}=0.005$, respectively), they had a significantly lower recurrence rate $(16 \%$ vs $23 \%, \mathrm{P}=0.044)$. While tumors with only papillary showed poor prognosticators including tumor size $>6 \mathrm{~cm}$,advanced stage,deep MI,necrosis,MELF pattern and LVI( $\mathrm{P}=0.003, \mathrm{P}=0.001, \mathrm{P}=0.05, \mathrm{P}=0.001, \mathrm{P}=0.001$ and $\mathrm{P}=0.001$ ), combining both $\mathrm{MUC}$ and papillary features showed significantly lower recurrence rate $(14 \%$ vs $23 \%, \mathrm{P}=0.009)$. Although not significant, the presence of MELF in a separate analysis of patients with absence of MUC-diff showed worse outcome(mean DFI:122 vs $138 \mathrm{mths}$ ).

\begin{tabular}{|l|c|l|c|c|}
\hline & Variable & $\begin{array}{l}\text { MUCabsent } \\
\text { N=366(\%)* }\end{array}$ & MUCpresentN=227(\%)* & P \\
\hline Stage & I-II III-IV & $239(65) 127(35)$ & $150(66) 77(34)$ & 0.859 \\
\hline MI & $<50 \% \geq 50 \%$ & $220(60) 145(40)$ & $129(57) 97(43)$ & 0.248 \\
\hline Necrosis & YesNo & $142(39) 223(61)$ & $113(50) 113(50)$ & 0.005 \\
\hline Papillary & YesNo & $201(55) 164(45)$ & $171(75) 56(25)$ & 0.001 \\
\hline MELF & YesNo & $159(46) 190(54)$ & $123(56) 98(44)$ & 0.020 \\
\hline Squamous diff & YesNo & $173(47) 193(53)$ & $118(52) 109(48)$ & 0.273 \\
\hline LVI & YesNo & $178(49) 187(51)$ & $120(53) 107(47)$ & 0.353 \\
\hline LN & YesNo & $88(24) 278(76)$ & $65(29) 162(71)$ & 0.247 \\
\hline Recurrence & YesNo & $82(23) 273(77)$ & $36(16) 188(84)$ & 0.044 \\
\hline * Some missing data & & \\
\hline
\end{tabular}

Conclusions: In this large series of LGEC, the presence of MUC-diff was associated with better outcomes despite the presence of poor prognostic factors including old age, papillary architecture and MELF pattern.

1094 Predictive Histologic Factors in Carcinosarcoma of the Uterus: A Multi-Institutional Study

Eman Abdulfatah, Leonardo Lordello, Muhammad Khurram, Kinda Havek, Koen Van de Vijver, Lamia Fathallah, Sudeshna Bandyopadhyay, Rouba Ali-Fehmi, Esther Oliva. WSU, Detroit, MI; MGH, Boston, MA; St.J, Detroit, MI.

Background: Uterine carcinosarcomas (CSs) are rare aggressive biphasic neoplasms. While studies support that carcinomatous components predict outcome, others do not. The aim of this study is to evaluate the clinical and histopathological features of a large multi-institutional cohort of CSs.

Design: A retrospective review of CSs ( $\mathrm{n}=196)$ from 1990 to 2012 at 3 institutions was conducted to analyze: histologic subtype, grade and \% of carcinomatous and sarcomatous components, presence of homologous/heterologous elements, necrosis, depth of myometrial invasion (MI), and histologic components upon recurrence. Data was analyzed using Cox-regression and Kaplan-Meier survival analyses.

Results: Patients' age ranged from 34-95 (median 68) years, being more common $>60$ years. Median tumor size was $7.0 \mathrm{~cm}$. Inner half MI was noted in $55 \%$, LVI in $64 \%$, adnexal involvement in $27.5 \%$ and lymph node (LN) metastasis in $27 \%$ of CSs. $40 \%$ of patients had stage I, $13 \%$ II, $30 \%$ III and $17 \%$ stage IV disease. Serous carcinoma was the predominant carcinomatous component $(51 \%)$, whereas heterologous elements $(64 \%)$, particularly rhabdomyoblastic differentiation (RMB)(71\%) was the most common sarcomatous component. $36 \% \& 66 \%$ of patients received adjuvant radiation or chemotherapy, respectively. Median disease free interval (DFI) and median overall survival(OS) were 11 and 16 months, respectively. Tumors $\geq 5 \mathrm{~cm}$, outer half MI, LVI, advanced stage and positive cytology were significantly associated with shorter DFI $(\mathrm{P}=0.039, \mathrm{P}=0.001, \mathrm{P}=0.015, \mathrm{P}=0.001$ and $\mathrm{P}=0.017$, respectively $)$ and worse 3-year $\mathrm{OS}(\mathrm{P}=0.003, \mathrm{P}=0.001, \mathrm{P}=0.005, \mathrm{P}=0.001$ and $\mathrm{P}=0.028$, respectively). Serous histology, and RMB had negative impact on 3 -year $\mathrm{OS}(\mathrm{P}=0.043$ and $\mathrm{P}=0.046$ respectively). In addition, sarcomatous histology upon recurrence and predominant sarcomatous component in primary tumor $(\geq 50 \%)$ showed shorter DFI ( $\mathrm{P}=0.009$ and $\mathrm{P}=0.027$, respectively). Cox regression analyses for DFI and 3-year OS are presented in Tables $1 \& 2$.

\begin{tabular}{|l|c|c|c|}
\hline Table 1 & & DFI HR $(95 \% \mathrm{CI})$ & P value \\
\hline Variable & $<50 \% \geq 50 \%$ & $12.00(1.01-4.01)$ & 0.05 \\
\hline Depth of MI & NegativePositive & $12.34(1.11-4.95)$ & 0.025 \\
\hline LN & $<50 \% \geq 50 \%$ & $12.45(1.21-4.94)$ & 0.012 \\
\hline$\%$ Of sarcomatous component &
\end{tabular}




\begin{tabular}{|c|c|c|c|}
\hline \multicolumn{4}{|l|}{ Table 2} \\
\hline Variable & & 3-year OS HR $(95 \% \mathrm{CI})$ & $P$ value \\
\hline Depth of MI & $<50 \% \geq 50 \%$ & $12.21(1.03-4.74)$ & 0.041 \\
\hline LN & NegativePositive & $12.81(1.40-5.67)$ & 0.004 \\
\hline RMB & $<50 \% \geq 50 \%$ & $12.73(1.28-5.81)$ & 0.009 \\
\hline
\end{tabular}

Conclusions: Our study supports that sarcomatous component particularly with RMB plays a prognostic role in CSs.

1095 Histomorphologic Study of Exogenous Testosterone Effects on the Ovaries and Uterus of Female to Male Transgender Patients

Ahmed A Abdulrahman, Scott D Richard, Robert T Ownbey, Suganthi Soundararajan. Drexel University College of Medicine, Philadelphia, PA; Hahnemann University Hospital, Philadelphia, PA.

Background: Administration of exogenous testosterone (T) to female-to-male transgender patients (FTMs) is key to their gender reassignment process. However, health issues may arise requiring regular monitoring. Androgen is thought to play a key role in follicular proliferation and growth. There are concerns that long-term T may induce polycystic ovarian syndrome (PCOS) which has been linked to increased risk of endometrial hyperplasia/carcinoma and ovarian carcinoma. Hence, hysterectomy and oophorectomy within 5 years of starting $\mathrm{T}$ is recommended. The purpose of this study is to describe the histologic effects of T on the ovaries and uterus of FTMs.

Design: Fourteen FTM patients who underwent hysterectomy and oophorectomy were retrospectively identified. All were placed on long-term $\mathrm{T}$ therapy and presented with pelvic pain and intermittent vaginal bleeding. Ages ranged from 22 to 41 (mean of 30). The duration of $\mathrm{T}$ therapy ranged from 2 months to 10 years (mean of 3 years and 9 months). Representative H\&E sections of the uterus and ovaries were reviewed by three of the authors. Gross findings were documented, including endometrial thickness and ovarian size and volume. Emphasis was placed on assessing the criteria for PCOS including thickening of the tunica albuginea, collagenization of the outer cortex, stromal hyperplasia, luteinization of stromal cells and cortical follicle cysts in various stages of development.

Results: All ovaries showed PCOS morphology in varying degrees, ranging from mild cortical thickening and stromal hyperplasia with at least one antral cystic follicle, to large ovaries with marked cortical thickening, stromal hyperplasia, luteinization of stromal cells as well as many relatively uniform cystic follicles arranged peripherally (up to 12/ovary, measuring 2-9 $\mathrm{mm}$ ) in all stages of growth except preovulatory stage indicating arrested growth (disordered follicle development). There were no corpora lutea identified indicating absent ovulation (except in 1 case where $\mathrm{T}$ was restarted 2 months prior, following 10 months cessation). The vast majority of cystic follicles were devoid of oocytes indicating increased loss. The endometria showed atrophic changes with no evidence of hyperplasia or carcinoma.

Conclusions: Administration of high-dose $\mathrm{T}$ to trans-men causes histomorphologic changes to the ovaries that closely resemble idiopathic PCOS, while the endometria showed atrophic changes with no evidence of hyperplasia or carcinoma.

1096 Androgen Receptor Expression, Androgen Receptor CAG Repeat Polymorphism and X-Chromosome Inactivation Patterns in Uterine Leiomyomas \& Leiomyosarcoma: Imunohistochemical and Molecular Study

Nisreen Abu Shahin, Amani Albustanji, Ali Alkhader, Mamoun Ahram. University of Jordan, Amman, Jordan.

Background: Uterine leiomyosarcoma (ULMS) and leiomyoma (ULM) are of smooth muscle origin, and may coexist in uterus. Rarity of ULMS caused limited genetic data of its carcinogenesis. Recently, androgen receptor (AR) is assumed to play role in many human diseases. This study aims to assess AR expression using immunohistochemistry, human androgen receptor gene assay (HUMARA) for X chromosome inactivation (XCI), and AR CAG repeat structure in ULM and ULMS.

Design: 46 ULM, 32 ULMS, and 24 samples of adjacent myometrial controls were tested. 5 patients had coexisting ULMS and ULM. IHC was reported as histologic score $((\mathrm{HS})=$ percentage of positive nuclei $\mathrm{X}$ stain intensity. XCI was investigated by HUMARA. CAG repeat lengths were evaluated in both alleles

Results: AR was expressed in $43.5 \%$ ULM and $31.3 \%$ ULMS ( $p=0.197$ ). 4 cases with coexisting tumors shared the same AR IHC profile (3-; 1+). Intra- tumor genetic heterogeneity (ITGH) was detected within AR CAG repeats in both tumors. Both tumors showed non-random XCI. 76.1\% ULM and 75\% ULMS ( $\mathrm{P}=0.26)$ showed CAG repeat variants.

\begin{tabular}{|l|l|l|l|}
\hline Parameter & ULMa & ULMSb & p-value \\
\hline AR expression & $43.5 \%$ & $31.3 \%$ & 0.197 \\
\hline low-expression $(\mathrm{HS}<6)$ & $76 \%$ & $90.6 \%$ & \\
& $(35 / 46)$ & $(29 / 32)$ & \\
\hline high-expression $(\mathrm{HS} \geq 6)$ & $24 \%$ & $9.4 \%$ & 0.087 \\
& $(11 / 46)$ & $(3 / 32)$ & \\
\hline Ratio of ITGH & $\begin{array}{l}76.1 \% \\
(35 / 46)\end{array}$ & $\begin{array}{l}75 \% \\
(24 / 32)\end{array}$ & 0.26 \\
\hline
\end{tabular}

In comparison with adjacent myometrium, 5/21 (23.8\%) of ULM showed new longer repeat variants, while $71.4 \%$ had no change; while $3 / 4$ ULMS displayed new shorter variants $(\mathrm{Chi} 2<0.001)$.

\begin{tabular}{|l|l|l|l|l|l|l|}
\hline Count & & \multicolumn{3}{|l|}{ CAG Repeat Variant Change } & \multirow{2}{*}{} & \multirow{2}{*}{$\begin{array}{l}\text { Pearson } \\
\text { Chi 2 }\end{array}$} \\
\hline \multirow{2}{*}{} & & $\begin{array}{l}\text { lelongated or } \\
\text { new longer } \\
\text { variants }\end{array}$ & shorter variants & Total & \\
\hline no chang & ULM & 15 & 5 & 1 & 21 & .001 \\
\cline { 2 - 6 } & ULMS & 0 & 1 & 3 & 4 & \\
\hline Total & & 15 & 6 & 4 & 25 & \\
\hline
\end{tabular}

Conclusions: Although no statistical association between AR expression and ULM or ULMS development was demonstrated, a third of studied cases expressed AR, potentially applicable in hormonal therapy. This is the first study to demonstrate ITGH of AR CAG repeats in ULM and ULMS; this needs further investigations for a role in carcinogenesis. No unique CAG repeats or common CAG repeats shared between ULM and ULMS, however, similar AR expression in coexisting ULM and ULMS may suggest malignant transformation in rare ULM into ULMS.

\section{Determining the Clinical Features and Histopathological Types} of Cervical Cancer in the Largest Tertiary Center in Ghana

Patrick K Akakpo, Leonard Derkyi-Kwarteng, Simon Naporo, Frederick Hobenu. University of Cape Coast, School of Medical Sciences, Cape Coast, Central Region, Ghana; KORLE-BU Teaching Hospital, Accra, Greater Accra, Ghana; 37 Military Hospital, Accra, Greater Accra, Ghana.

Background: Cervical Cancer is estimated to be the most frequent cancer among women in Ghana. With an estimated age standardized ratio (ASR) of 39.5 per 100,000 women. A 10 year (1991-2000) study of cancer mortality pattern revealed that cervical cancer was one of the four leading causes of cancer deaths in females in Ghana. There is currently no national cervical cancer screening program in Ghana.

Design: To determine the demographic, clinical features, geographical distribution, histopathological types and subtypes of cervical cancer in Ghana to inform a cervical cancer screening program. Biographical data, presenting signs, symptoms and histopathological type and subtypes of cervical cancer diagnosed were collected from request cards and histopathology reports and entered into EPI INFO. The data was analyzed to meet the objectives.

Results: A total of 1094 cases were reviewed. $81.6 \%$ of the cases were malignant. These were made up of squamous cell carcinomas (69.8\%), adenocarcinomas (5\%), adenosquamous carcinomas $(1.6 \%)$ and a group of carcinomas that did not fit any of the stated morphological classes $(5.2 \%) .50 .2 \%$ of patients were in the age range 41-60years followed by $27.6 \%$ in the range $61-80$ years and then $18.5 \%$ between the ages $21-40$ years. $3.4 \%$ were older than 80 years and one patient was younger than 21 years. $52.8 \%$ of patients presented with postmenopausal bleeding, this was followed by those with offensive vaginal discharge $12.1 \%$ and $6.7 \%$ with menstrual disorders. $81.7 \%$ of patients presented more than 1 month after onset of symptoms.

Conclusions: Squamous cell carcinoma is the commonest cervical cancer in this study. Cervical cancer occurs in a significant number of patients below 40 years though most patients are older than 40years. Patients commonly present with post-menopausal bleeding and offensive vaginal discharge more than a month after symptom onset, suggesting late presentation.

1098 Adenocarcinoma of Cervix Is Surging: Is High-Risk HPV Subtype(s) Infection Pattern Changing in a Cervical Carcinoma Prevalent Developing Country? A Retrospective PCR-Based Study

Shabnam Akhter, Mohammed Kamal, Ashim R Barua, C James Sung, W Dwayne Lawrence, $M$ Ruhul Quddus. BSM Medical University, Dhaka, Bangladesh; Women \& Infants Hospital/Alpert Medical School of Brown University, Providence, RI.

Background: Cervical carcinoma is the second most frequent cancer in Bangladeshi women and it has the $4^{\text {th }}$ highest rate of cervical cancer deaths in the world. The majority of cancers are still squamous cell type however, the glandular neoplasms are rising. HPV vaccination program is new and limited in the country. Identification of the prevalence of various high-risk HPV (hrHPV) subtypes in cervical adenocarcinoma will fill up the void in epidemiologic data and prepare for the preemptive strategies, should or when appropriate, to control the disease.

Design: After IRB approval, 71 cases of cervical invasive and in-situ adenocarcinoma (AdCa/AIS) were retrieved from the pathology department, BSM Medical University, Dhaka, Bangladesh. DNA was extracted from representative tissues using QIAmp DNA FFPE Kit. DNA was then tested for high-risk HPV using GenomeMe ${ }^{\mathrm{TM}}$ 's GeneNav ${ }^{\mathrm{TM}}$ HPV One qPCR Kit with known positive controls. This kit detected HPV 16 and HPV 18 and non-specifically other 12 hrHPVs (HPV 31, 33, 35, 39, 45, 51, 52, 56, $58,59,66$ and 68 ). Afterwards non-specific hrHPV positive samples were tested by GenomeMe $^{\mathrm{TM}}$ 's GeneNav ${ }^{\mathrm{TM}}$ HPV Genotyping qPCR Kit (GenomeMe, Richmond, BC, Canada) to identify all $14 \mathrm{hrHPV}$ individually. The tests were run on Bio-RAD CFX Touch qPCR instrument.

Results: DNA of all 71 cases was successfully amplified. The Table 1 shows distribution of hrHPV. 


\begin{tabular}{|c|c|}
\hline High-risk HPV Subtypes & Total cases (N=71)(\%) \\
\hline HPV 16 & $45(63.4)$ \\
\hline HPV 18 & $12(16.9)$ \\
\hline HPV 33 & $1(1.4)$ \\
\hline HPV 16/18 & $6(8.5)$ \\
\hline HPV 16/33 & $2(2.8)$ \\
\hline HPV 16/39 & $1(1.4)$ \\
\hline HPV 16/45 & $2(2.8)$ \\
\hline HPV 16/59 & $1(1.4)$ \\
\hline HPV 16/18/33 & $1(1.4)$ \\
\hline
\end{tabular}

Conclusions: A narrow spectrum of hrHPV subtypes are found in AdCa/AIS cases in this study with HPV 16 being the most dominant (63.4\%) subtype; a distant second is HPV $18(16.9 \%)$. This is in contrast to the western countries where HPV 18 was reported to be most frequent ( $52 \%$ to $56 \%$ ) subtype. HPV 33 was more common in Bangladesh compared to HPV 45 . HPV 45 has been reported to be more common in western world (3.4\% to $10 \%$ ). The significance of finding $88.7 \%$ being hrHPV $16 / 18$ in Bangladeshi women is enormous as the currently available bivalent HPV vaccine administered in the country covers both subtypes. Nevertheless it is important to keep in mind that rare subtypes exist and may become dominant due to the effects of vaccination.

1099 Comparison of the Frequency of Multi-Viral and RareSubtype(s) High-Risk HPV Infection in Glandular and Squamous Lesions of the Uterine Cervix in a Cervical Carcinoma Prevalent Country: A Retrospective PCR-Based Study

Shabnam Akhter, Mohammed Kamal, C James Sung, W Dwayne Lawrence, M Ruhul Quddus. BSM Medical University, Shahbag, Dhaka, Bangladesh; Women \& Infants Hospital/Alpert Medical School of Brown University, Providence, RI.

Background: The frequency of multi-viral and rare high-risk HPV (hrHPV) subtype infection is on the rise. Vaccination against most frequently encountered hrHPV may change infection patterns and rare subtypes may become prevalent in future. Knowledge of the prevalence of existing hrHPV in a region is important. Bangladesh, a densely populated country of 160 million and nearly half being women, has a high incidence of cervical cancer related deaths, where vaccination has recently started. We are comparing the frequency of multi-viral and rare subtype(s) hrHPV infection in glandular and squamous lesions of the uterine cervix.

Design: Seventy one cervical invasive and in-situ adenocarcinoma and 39 cases of high grade SIL were retrieved from BSM Medical University, Dhaka Bangladesh after IRB approval. DNA was extracted by using QIAam DNA FFPE Kit. Samples were tested using GenomeMe ${ }^{\mathrm{TM}}$ 's GeneNav ${ }^{\mathrm{TM}}$ HPV One qPCR Kit. This kit detected HPV 16 and HPV 18 and non-specificically other 12 hrHPVs. Then non-specific hrHPV positive samples were tested by GenomeMe ${ }^{\mathrm{TM}}$ 's GeneNav ${ }^{\mathrm{TM}} \mathrm{HPV}$ Genotyping qPCR Kit (GenomeMe, Richmond, BC, Canada) to identify all $14 \mathrm{hrHPV}$ individually. The tests were run on Bio-RAD CFX Touch qPCR instrument.

Results: Of the 110 cases, 72 samples were positive for HPV 16 and 13 for HPV 18 . The remaining 25 cases were positive for other subtypes and the genotyping results are shown in Table 1.

\begin{tabular}{|c|c|c|c|c|}
\hline $\begin{array}{l}\text { HPV } \\
\text { Subtypes }\end{array}$ & $\begin{array}{c}\text { Glndular } \\
\text { Lesions } \\
(\mathrm{N}=71)(\%)\end{array}$ & $\begin{array}{c}\text { Squamous } \\
\text { Lesions } \\
(\mathrm{N}=39)(\%)\end{array}$ & $\begin{array}{l}\% \text { of Rare Subtype } \\
\text { in Glandular } \\
\text { Lesions }(\mathrm{N}=71)\end{array}$ & $\begin{array}{l}\% \text { of Rare Subtype } \\
\text { Infection in Squamous } \\
\text { Lesions }(\mathrm{N}=39)\end{array}$ \\
\hline HPV 31 & 0 & 1 & \multirow{11}{*}{ 6/71 (8\%) } & \multirow{11}{*}{ 10/39 (27\%) } \\
\hline HPV 33 & 1 & 1 & & \\
\hline HPV 67 & 0 & 1 & & \\
\hline HPV 16/18 & $6(8.5 \%)$ & $2(5 \%)$ & & \\
\hline HPV 16/33 & $2(3 \%)$ & 1 & & \\
\hline HPV 16/35 & 0 & 1 & & \\
\hline HPV 16/39 & 1 & 1 & & \\
\hline HPV 16/45 & $2(3 \%)$ & 0 & & \\
\hline HPV 16/52 & 0 & 1 & & \\
\hline HPV 16/59 & 1 & 0 & & \\
\hline HPV 116/68 & 0 & 1 & & \\
\hline
\end{tabular}

One triple and one quadruple hrHPV infection are noted in glandular and squamous lesion each respectively.

Conclusions: Infection by rare subtype hrHPVs is three times more common in squamous compared to glandular lesions. The frequency of multi-viral infection is similar in both groups. Vaccination against a wide range of rare subtype(s) hrHPV is needed to controlling squamous lesions compared to glandular lesions.

\section{PD-L1 Frequently Expressed in Endometrial Carcinoma} Associated with Mismatch-Repair Deficiency

Omar Al-Nourhji, Gloria Zhang, Youran Zou, Charles V Biscotti, Peter Rose, Bin Yang. Cleveland Clinic, Cleveland, $\mathrm{OH}$.

Background: Immunoinhibitory molecule PD-L1 suppresses T cell activation, leading to tumor progression. Studies have shown that PD-L1 expression in certain cancers associates with a favorable response to immunotherapy. A recent clinical trial shows that patients with mismatch-repair (MMR) deficient colorectal cancer respond to immune checkpoint blockade pembrolizumab significantly better than those with MMR proficient cancers. We compared PD-L1 expression in a large cohort of both MMR deficient and MMR proficient endometrial carcinomas.

Design: A total of 212 endometrial cancer cases were identified in our pathology database. Among these cases, 170 had hysterectomy specimens, staging and tissue blocks sufficient for inclusion in the study. All cases had MMR immunohistochemistry with 4 antibodies (MLH1, PMS2, MSH2 and MSH6) and MLH1 methylation and germline mutation testing when indicated. Immunohistochemistry with PD-L1 antibody (Clone SP263 from Vantana) was performed on tumor recuts. PD-L1 expression in tumor infiltrating lymphocytes (TIL) was graded as negative, $1+(<5 \%), 2+(5-30 \%)$ and $3+(>30 \%$ of TIL $)$.

Results: Among 170 cases, 115 expressed all 4 MMR proteins, 49 had MLH1 promoter methylation and 6 had confirmed MMR germline mutations. Overall, approximately $51 \%$ of all endometrial carcinomas expressed PD-L1. Any expression of PD-L1 (1-3+) occurred in $35.7 \%$ of MMR-positive, $79.6 \%$ of MLH1 methylated and $100 \%$ of Lynchrelated cases. Higher expression level of PD-L1 (2-3+) occurred in 17.4\% of MMRpositive, $55.1 \%$ MLH1 methylated and $100 \%$ Lynch-related cases. PD-L1 expression significantly differed between MMR deficient $(81.8 \%)$ and MMR proficient $(35.7 \%)$ cases $(\mathrm{p}<0.001)$. Among cancer cell types, PD-L1 was more frequently expressed in clear cell carcinoma (100\%) than serous $(33.3 \%)$ and endometrioid (49\%).

Conclusions: PD-L1 was expressed in the vast majority of MMR deficient endometrial carcinomas, including $100 \%$ of Lynch syndrome and $80 \%$ of MLH1 methylated cases, and minority of MMR proficient carcinomas. Assessment of PD-L1 expression by immunohistochemistry might help guide potential immunotherapy in endometrial cancer.

1101 Small Cell Neuroendocrine Carcinoma of the Uterine Cervix: A Review of 226 Cases with Emphasis on Patterns of Growth and Immunohistochemical Features

Isabel Alvarado-Cabrero, Raquel Valencia-Cedillo, Emeli Dominguez, Delia PérezMontiel. Hospital de Oncología, CMN Siglo XXI IMSS, Mexico City, Mexico; Instituto Nacional de Cancerología, Mexico City, Mexico.

Background: Small cell neuroendocrine carcinoma (SNEC) is a rare neoplasia that comprises $1-6 \%$ of cervical tumors. The vast majority of cases can be diagnosed on $\mathrm{H} \& \mathrm{E}$ alone, however in problem cases,

Immunohistochemistry can be very helpful in making distinction from other tumors. On the other hand, most patients are diagnosed based on a small biopsy.

Because of the rarity of SNEC, the pathologic and immunohistochemical features have not yet been fully elucidated.

Design: We conducted a retropective review to explore the patterns of growth of SNEC and their immunohistochemical features.

All patients with SNEC of the cervix diagnosed between 1995-2014 were identified from tumor registry databases at two Cancer Centers. The pathological review was conducted by 2 pathologists specializing in ginecologic cancer. Immunohistochemical analysis was performed in all cases using several markers including chromogranin A, synaptophysin, TTF1 and CD56.

By re-evaluating 226 SNEC of the cervix, we documented 11 patterns of growth: solid (SOL), trabecular (TRB), Nesting (NES), Glandular(GL), Insular (INS), Linear (LIN), spindle cell (SC), Isolated small nests (ISN), hemangiopericytoma-like (HPL) , paraganglioma-like (PRGL) and adenoid basal-like (ABL).

Results: We found $65(28 \%)$ tumors to be SOL, 31(13\%) INS, 30(13\%) TRB, $24(11 \%)$ NES, 20(9\%) GL, 17(8\%) LIN, 15 (7\%) SC, 10(4\%) ISN, 6(3\%) ABL, 4(2\%) PRGL and $4(2 \%)$ to be HPL. There was coexisting adenocarcinoma in 14 cases, squamous cell carcinoma in 8 cases, adenocarcinoma in situ in 6 cases and squamous cell carcinoma in situ in 3 cases.

$85 \%$ of the tumors were synaptophysin,$+ 65 \%$ were chromogranin $\mathrm{A}+, 55 \%$ were TTF $1+$ and $46 \%$ were CD $56+$

Conclusions: SNEC of the cervix can manifest a variety of different growth patterns. A panel of immunostains that contains synaptophysin, chromogranin A, TTF1 and CD56 usually suffices to confirm or refute a diagnosis of this neoplasm.

1102 Trefoil Factor Family 2 Protein: A Promising Immunohistochemical Marker for Diagnosing Lobular Endocervical Glandular Hyperplasia and Gastric-Type Adenocarcinoma of Uterine Cervix Shiho Asaka, Hiroyoshi Ota. Shinshu University Hospital, Matsumoto, Nagano, Japan; Shinshu University School of Medicine, Matsumoto, Nagano, Japan.

Background: Gastric-type adenocarcinoma (GA) is a subtype of uterine cervical adenocarcinoma with worse prognosis than the usual type endocervical adenocarcinoma (UEA). Lobular endocervical glandular hyperplasia (LEGH) is a rare lesion of uterine cervix that simulates gastric pyloric mucosa and may represent a precursor lesion to GAs. Because of the morphological similarity of GAs to UEA and LEGH to normal endocervical gland, immunohistochemical markers for gastric gland mucins (MUC6 and $\alpha 1,4$-linked $\mathrm{N}$-acetylglucosamine residues ( $\alpha$ GlcNAc-R) recognized by HIK 1083 antibody) have been introduced. However, MUC6 is also expressed in normal endocervical glands and HIK1083 antibody has limited availability. Here we evaluated the utility of immunostaining for trefoil factor family 2 protein (TFF2) for detecting gastric immunophenotype in GAs and LEGH.

Design: We performed TFF2 and aGlcNAc-R immunostaining in 69 cases of endocervical glandular lesions: 15 LEGH, 24 adenocarcinoma in situ (AIS), and 30 invasive adenocarcinoma (IAC). Positive staining was scored semiquantitatively as 0 (negative), $1(5 \%-25 \%), 2(25 \%-50 \%), 3(50 \%-75 \%)$, and 4 (more than $75 \%$ ).

Results: TFF2 and $\alpha$ GlcNAc-R immunoreactivity were present in the cytoplasm and in the luminal secretions in LEGH, AIS and IAC but not in normal endocervical glands. The frequency of TFF2 expression was highly concordant with that $\alpha$ GlcNAc-R: $15(100 \%)$ vs. $15(100 \%)$ LEGH cases, $10(41.7 \%)$ vs. $9(37.5 \%)$ AIS cases, and 13 (43.3\%) vs. $13(43.3 \%)$ IAC cases. The staining scores for TFF2 showed a significant correlation with those given for $\alpha \mathrm{GlcNAc}$ [ $(r=0.91$ in LEGH, $r=0.93$ in AIS, $r=0.98$ 
in IAC $(\mathrm{P}<0.01)]$. AIS and IAC patients with higher TFF2 expression (score 2-4) had impaired overall survival and disease-free survival compared with patients with lower TFF2 expression (score $0-1)(\mathrm{P}<0.01)$.
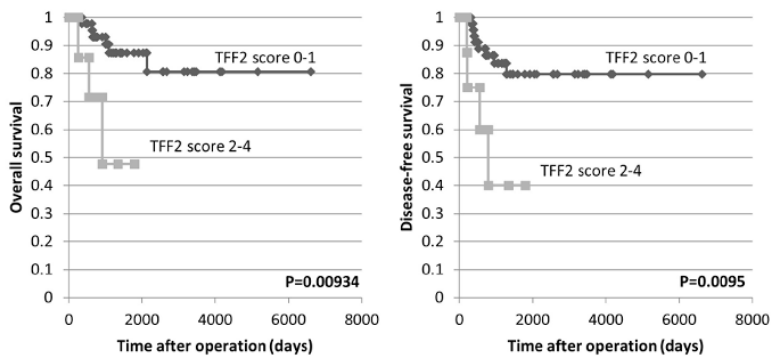

Conclusions: TFF2 is a promising immunohistochemical marker, and determination of TFF2 in uterine cervical secretion also may be useful for diagnosing LEGH and GAs.

1103 Prognostic Significance of PD-1 and PDL-1 Expression in Ovarian Carcinomas and Tumor Associated Immune Cells

Binara Assylbekova, Jose P Leone, Kate Serdy, Xin Li, Kavita R Varma, Esther Elishaev, Beth Z Clark, David J Dabbs, Rohit Bhargava. Magee Womens Hospital of UPMC, Pittsburgh, PA; University of Iowa, Iowa City, IA.

Background: Programmed cell death 1 (PD1), immuno-inhibitory receptors on T-cell and their ligands (PDL-1/2) on tumor cells, is an emerging cancer regulatory mechanism of prognostic and therapeutic potential. PD-1 and PDL-1 immunohistochemical expression in tumor cells and associated immune cells may provide useful theranostic information.

Design: In this study we report expression of PD-1 and PDL-1 in ovarian carcinoma epithelium (PD-1epi and PDL-1epi) and in associated immune cells if present (PD$1 \mathrm{imm}$ and PDL-1imm), and correlate those with survival. Due to only weak reactivity in most cases, an $\mathrm{H}$-score of 1 was considered a positive result.

Results: PDL-1 expression is membranous in tumor cells. PDL-1 and PD-1 expression in immune cells and PD-1 in tumor epithelium is cytoplasmic and membranous. Overall, the expression for all the markers was weak and focal/patchy. The expression pattern with respect to tumor type is shown in table 1 .

\begin{tabular}{|l|l|l|l|}
\hline & Non-serous & Serous & p-value \\
\hline PD-L1epi+ & $\begin{array}{l}14 / 34(41 \%) \\
\text { Mean H-score 15 }\end{array}$ & $\begin{array}{l}\text { 35/55 (64\%) } \\
\text { Mean H-score 11 }\end{array}$ & 0.0494 \\
\hline PD-L1imm+ & $\begin{array}{l}21 / 33(64 \%) \\
\text { Mean H-score 44 }\end{array}$ & $\begin{array}{l}48 / 55(87 \%) \\
\text { Mean H-score 45 }\end{array}$ & 0.0149 \\
\hline PDlepi+ & $\begin{array}{l}1 / 34(3 \%) \\
\text { H-score 35 }\end{array}$ & $\begin{array}{l}\text { 0/56 (0\%) } \\
\text { H-score NA }\end{array}$ & 0.3778 \\
\hline PDlimm+ & $\begin{array}{l}22 / 34(65 \%) \\
\text { Mean H-score 47 }\end{array}$ & $\begin{array}{l}50 / 56(89 \%) \\
\text { Mean H-score 41 }\end{array}$ & 0.0067 \\
\hline
\end{tabular}

PD-1 and PDL-1 expression in immune cells and PDL-1 expression in tumor cells is more frequent in serous tumors. However, only $2 \%$ of the cases showed $\geq 50 \%$ tumor cell positivity with PDL-1 (a requirement to receive pembrolizumab in lung cancer) and none of the cases showed diffuse strong reactivity. There was no significant difference in OS based on PD-1 and PDL-1 positivity (figure 1).

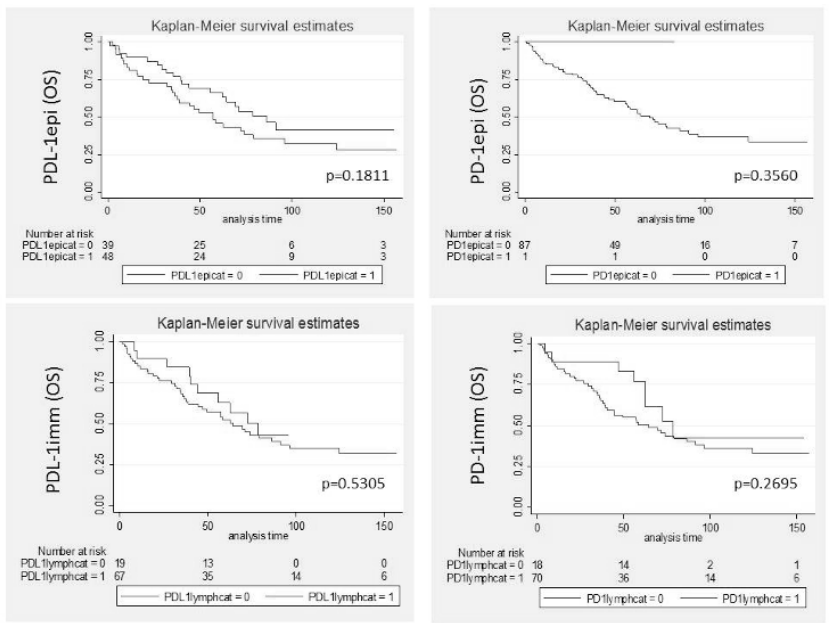

DFS was worse for tumors with PD-1 expression in immune cells $(\mathrm{p}=0.0461)$. Multivariate analysis of known prognostic factors showed worse survival for high FIGO stage, advanced age and for patients who did not receive chemotherapy.

Conclusions: PD-1 and PDL-1 expression is generally not prognostic in ovarian cancer except for PD-1 reactivity in immune cells. The often negative or weak IHC expression for PD-1 and PDL-1 suggests limited utility of immune check point inhibitors in ovarian cancer.
1104

PAX8, SOX10 and GATA3 Immunostains: Friend or Foe in the Diagnosis of Vulvar Paget Disease?

Marina K Baine, Pei Hui, Natalia Buza. Yale University, Yale New Haven Hospital, New Haven, CT.

Background: Evaluation of vulvar Paget disease (PD) includes the differential diagnosis of melanoma and pagetoid spread from urothelial or colorectal neoplasms. While the utility of some immunohistochemical markers has been well established, others remain to be assessed in larger patient cohorts. PAX8, a sensitive Mullerian marker, has not been examined in vulvar PD. SOX10, a melanocytic marker routinely used for melanocytic lesions, has recently also been shown to be expressed in a subset of breast carcinomas. Vulvar PD, which is known to stain with certain breast markers (i.e. GCDFP-15), has not yet been evaluated for SOX10 expression. GATA3, a sensitive marker of urothelial carcinoma, if also specific, could help distinguish between primary vulvar PD from secondary pagetoid spread of urothelial neoplasia. Our aim was to determine the diagnostic utility of PAX8, SOX10, and GATA3 in the diagnosis of primary vulvar PD and its distinction from melanoma and secondary involvement by urothelial carcinoma. Design: A tissue microarray was constructed, composed of duplicate cores of primary and recurrent vulvar PD from 40 patients, and was stained with PAX8, SOX10, GATA3, Cytokeratin-7 (CK7), Cytokeratin-20 (CK20) and p63 immunostains.

Results: After excluding histospots with insufficient tissue, complete immunoprofile was established for 23 of 40 women, with an age range between 42 and 99 years old. Five of these women had invasive disease and two had one or more recurrences. All cases of vulvar PD were negative for PAX8 and SOX10 immunostains, while GATA3 was strongly positive in all but one case $(96 \%)$. In addition, all cases were positive for CK7 and negative for CK20 and p63 immunostains.

\begin{tabular}{|c|c|c|c|c|c|c|}
\hline & PAX8 & SOX10 & GATA3 & CK7 & CK20 & P63 \\
\hline $\begin{array}{c}\text { Vulvar PD } \\
n=23\end{array}$ & $0 / 23(0 \%)$ & $0 / 23(0 \%)$ & $\begin{array}{c}22 / 23 \\
(96 \%)\end{array}$ & $\begin{array}{c}23 / 23 \\
(100 \%)\end{array}$ & $0 / 23(0 \%)$ & $0 / 23(0 \%)$ \\
\hline
\end{tabular}

Conclusions: Unlike most Mullerian epithelial tumors, vulvar PD is negative for PAX8 immunostain, presenting a potential diagnostic pitfall. GATA3 is positive in most cases of primary vulvar PD, and should not be misinterpreted as evidence of pagetoid spread from a urothelial primary. A CK 7 positive/ CK20 negative/ p63 negative immunoprofile is helpful in ruling out urothelial origin. SOX10 expression is absent in vulvar $\mathrm{PD}$, therefore it proves to be an additional useful marker for distinction of vulvar PD from melanoma.

1105 Extrapelvic Metastatic Endometrial Stromal Sarcoma: A BiInstitutional Review

Nick Baniak, Scott Adams, Rajni Chibbar, Rani Kanthan. University of Saskatchewan, Saskatoon, SK, Canada.

Background: Endometrial stromal sarcoma (ESS) is rare, comprising $0.2 \%$ of all uterine malignancies. In this case series we provide insight into the unique occurrence of extrapelvic metastatic ESS.

Design: A 19-year (1997-2015) retrospective review of ESS was conducted in the Laboratory Information system at two institutions [Regina \& Saskatoon], with particular attention to initial diagnosis and metastatic behaviour.

Results: Thirty-two patients with an established diagnosis of ESS were identified. The mean age at first diagnosis was 50.2 years (range 20-88). Extrapelvic metastases were noted in 10 patients. In these patients, the identified primary site was uterine $(n=9)$ and ovarian $(\mathrm{n}=1)$. There was no correlation between the grade of the primary tumour and metastatic behaviour. Most common sites of metastasis were lung $(n=9)$, small or large bowel $(n=4)$, liver $(n=3)$, and abdomen not otherwise specified $(n=1)$. Two cases of ESS presented initially with an extrapelvic metastasis with no prior history of uterine/ ovarian ESS. Of the remaining cases, the mean time from initial diagnosis of ESS to first presentation of extrapelvic metastases was 10.4 years (range $0.75-20$ years). Multiple recurrences of extrapelvic metastatic ESS were noted in four patients.

Conclusions: Extrapelvic metastases of ESS to the lung, liver, or bowel may occur as a delayed event; thus, clinical awareness of this unusual neoplasm is necessary for accurate diagnosis. In cases with no documented primary lesion, the possibility of $d e$ novo ESS arising within unusual endometriotic foci must be considered. The biological behaviour of ESS continues to pose both clinical and pathological diagnostic challenges.

1106 High-Grade Endometrial Carcinomas Show Frequent Aberrant Expression of Yolk Sac Markers

Keegan Barry-Holson, Chieh-Yu Lin, Ann Folkins. Stanford University School of Medicine, Stanford, CA.

Background: Small series and case reports describe extragonadal pelvic yolk sac tumors occurring in the endometrium. We have similarly noted expression of yolk sac immunohistochemical markers in four index cases of high-grade endometrial carcinoma. However, it is unclear whether these cases represent true yolk sac tumors or simply reflect aberrant expression of yolk sac markers. The aim of our study was to retrospectively determine how often yolk sac markers are expressed in endometrial carcinomas.

Design: The pathology database was searched from 1997 to 2016 for cases of high-grade endometrial carcinoma ( 20 cases: 6 clear cell, 3 endometrioid, 3 serous, 3 high-grade NOS, 2 dedifferentiated, 1 undifferentiated, and 2 mixed carcinomas) and low-grade endometrioid carcinoma ( 7 cases). Immunohistochemical stains for glypican-3 and SALL4 were performed on selected tissue blocks from the high-grade cases. Only SALL4 was performed on the low-grade carcinomas. Three pathologists scored the stains. Our four index cases of high-grade endometrial carcinoma expressing yolk sac markers were also included.

Results: None of the low-grade endometrioid tumors stained with SALL4 while almost a third of the retrospective high-grade carcinomas were at least focally positive (see 
table). The index cases were identified by glandular and solid architecture with unusual supra- and subnuclear vacuoles; these all showed patchy but strong SALL4 staining. In the retrospective cases, SALL4 expression was not confined to a specific high-grade tumor type and did not correlate with this yolk-like morphology. Focal SALL4 staining was seen in serous, clear cell, endometrioid, and undifferentiated carcinomas. The one case with patchy strong staining was the undifferentiated component of a dedifferentiated carcinoma. Glypican-3 expression was present in 4/15 retrospective high-grade cases, 2 of which also showed SALL4 expression (see table).

\begin{tabular}{|l|l|l|}
\hline Endometrial tumor type & SALL4 & Glypican-3 \\
\hline High-grade carcinomas (16 retrospective cases) & $5 / 16$ & $4 / 15$ \\
\hline High-grade carcinomas (4 index cases) & $4 / 4$ & $2 / 2$ \\
\hline Low-grade endometrioid carcinoma (7 retrospective cases) & $0 / 7$ & N/A \\
\hline
\end{tabular}

Conclusions: A significant proportion of high-grade endometrial carcinomas, regardless of subtype or morphologic evidence of yolk sac-like features, demonstrate expression of yolk sac immunohistochemical markers. This suggests that SALL4 and glypican-3 expression may represent aberrant expression of a primitive marker in high-grade carcinomas rather than true yolk sac differentiation.

1107 Implementation of Somatic BRCA Testing for Ovarian Carcinoma in Routine Practice

Beryl Bayol, Frederique Penault-Llorca, Yannick Bidet, Anne Cayre, Mathilde GayBellile, Christophe Pomel, Sandrine Viala, Marie-Ange Mouret-Reynier, Lucie Tixier Yves-Jean Bignon. Centre Jean Perrin, Clermont Ferrand, France.

Background: As patients with high grade serous ovarian, Fallopian tube and primary peritoneal cancers with either germinal or somatic $B R C A$ pathogenic variants are eligible to PARP inhibitors treatment, screening for somatic $B R C A$ alterations are becoming a routine test. However, extracted DNA from FFPE tissues tend to be highly fragmented and in limited quantity, making analysis of large genes such as $B R C A 1$ and $B R C A 2$ complex. We examined the feasibility of analyzing DNA extracted from formalin-fixed paraffin-embedded (FFPE) ovarian, Fallopian tube and primary peritoneal cancers tissues to identify significant somatic variants in $B R C A$ genes by Next Generation Sequencing (NGS)

Design: Pts: 57 cases (primitive tumor, peritoneal carcinomatosis or metastasis) including for this pilot study, 8 patients with known constitutional pathogenic variants and 4 variants of uncertain significance (VUS). 29/57 of the samples were collected after chemotherapy. Methods: PCR multiplex (Multiplicom) to generate short amplicons, Miseq ${ }^{\circledR}$ sequencing with an Illumina ${ }^{\circledR}$ kit. Analysis: Seq Next and Mutect softwares. Results: Only 17\% DNA extracted were of good quality or slightly fragmented. Among 57 cases, only 2 couldn't be sequenced and 2 were uninterpretable (bad amplification of highly fragmented DNA). Most patients had previously benefited from a constitutional mutation research on BRCA genes. Confirmation of all constitutional variants by somatic NGS analysis (pathogenic variants, VUS and polymorphisms). New findings: 8 somatic variants, $(14 \%) ; 5$ being pathogenic and confirmed by Sanger sequencing. Conclusions: This study confirmed that detection of BRCA $1 / 2$ somatic variants in FFPE tissues using commercially available technology is a reliable, rapid (15 days), robust and efficient technology, in spite of the important length of zones to be analyzed. Several changes in surgical and pathological pratices have been implemented to enhance specimen collection and DNA quality.

1108 DICER1 Mutations in Müllerian Adenosarcomas

Gregory Bean, Gregor Krings, Karuna Garg. UCSF, San Francisco, CA.

Background: There is currently limited information on the genomic landscape of Mullerian adenosarcoma (MA). The aim of our study was to assess the molecular abnormalities in MA, with particular emphasis on tumors with stromal overgrowth (SO) and/or rhabdomyosarcomatous (rhabdo) differentiation.

Design: DNA was extracted from 14 cases of MA (5 with rhabdo, 4 with SO) and matched normal tissue. Capture-based next generation sequencing was performed using an assay that targets the coding regions of 510 cancer genes and 40 introns. Duplicate reads were removed computationally for allele frequency determination and copy number calling. Single nucleotide variants, insertions/deletions and copy number alterations (CNA) were evaluated.

Results: DICER 1 mutations were identified in $5 \mathrm{MA}, 3$ with rhabdo and 2 without. Four had 2 DICER1 mutations and the other had 4; these were too far apart to determine if in cis or trans. Activating hotspot mutations were identified in PIK3CA (2/14), KRAS (1/14) and NRAS (1/14); PIK3CA and RAS mutations were mutually inclusive. Pathogenic variants of tumor suppressors FBXW7 (2/14) and TP53 (2/14) and deep deletions of BAP1 (2/14) were also identified. One tumor had CDK4/MDM2 amplification. CNA were variable and included chr8 gain in 6 cases.

\begin{tabular}{|l|l|l|l|l|l|l|}
\hline $\begin{array}{l}\text { Case } \\
\#\end{array}$ & Age & Stage & $\begin{array}{l}\text { Follow Up } \\
\text { (months) }\end{array}$ & Rhabdo & $\begin{array}{l}\text { Stromal } \\
\text { Overgrowth }\end{array}$ & $\begin{array}{l}\text { Pathogenic/Likely } \\
\text { Pathogenic Aberrations }\end{array}$ \\
\hline 1 & 40 & IA & NED (6) & yes (E) & no & $\begin{array}{l}\text { DICER1 p.G1809R, } \\
\text { p.R392* }\end{array}$ \\
\hline 2 & 38 & IB & NED (9) & yes (E) & no & $\begin{array}{l}\text { DICER1 p.E1813G, } \\
\text { p.H1767fs }\end{array}$ \\
\hline 3 & 35 & IA & NED (178) & yes (E) & yes & $\begin{array}{l}\text { DICER1 p.D1709N, } \\
\text { p.L1094*, FBXW7 } \\
\text { p.R505G }\end{array}$ \\
\hline 4 & 37 & IB & NED (103) & no & no & $\begin{array}{l}\text { DICER1 p.820_822del, } \\
\text { p.D1810H }\end{array}$ \\
\hline 5 & 92 & IB & - & no & yes & $\begin{array}{l}\text { DICER1 p.E1813K, } \\
\text { p.P1366L, p.R404I, } \\
\text { p.Y876S, CTCF p.L66fs, } \\
\text { CTCF p.R457*, DDX3X } \\
\text { p.W60*, NRAS p.G13R, } \\
\text { NSD1 p.R1914C, PIK3CA } \\
\text { p.H1047Y, SETD2 p.L959fs }\end{array}$ \\
\hline 6 & 47 & IA & NED (99) & yes (E) & no & $\begin{array}{l}\text { FBXW7 p.R505L, KMT2D } \\
\text { p.R2471*, TP53 p.R248W }\end{array}$ \\
\hline 7 & 61 & IB & NED (4) & yes (P) & yes & TP53 p.A138P \\
\hline 8 & 62 & III-IV & deceased (34) & no & yes & BAP1 homozygous deletion \\
\hline 9 & 46 & IB & NED (5) & no & no & BAP1 homozygous deletion \\
\hline 10 & 73 & IA & NED (57) & no & no & CDK4/MDM2 amplification \\
\hline 11 & 66 & IA & NED (22) & no & no & $\begin{array}{l}\text { ARHGAP35 p.F73fs, } \\
\text { ARID1A p.G92fs, COL2A1 } \\
\text { p.A791fs, KRAS p.G12V, } \\
\text { PIK3CA p.G118D }\end{array}$ \\
\hline 12 & 23 & - & NED (3) & no & no & - \\
\hline 13 & 44 & IA & $\begin{array}{l}\text { no residual } \\
\text { tumor }\end{array}$ & no & no & - \\
\hline 14 & 60 & IA & - & no & no & - \\
\hline
\end{tabular}

$\mathrm{E}=$ embryonal; $\mathrm{P}=$ pleomorphic

Conclusions: MA showed frequent DICER1 mutations, including but not restricted to MA with rhabdo. A subset of MA harbor mutations in PIK3CA and RAS pathway. Recurrently inactivated tumor suppressors include BAP1 and TP53. CNA are variable but include frequent chr8 gain.

1109 Inflammatory Myofibroblastic Tumor of the Uterus: A Clinicopathological, Immunohistochemical, and Molecular Analysis of 13 Cases

Jennifer Bennett, Valentina Nardi, Marjan Rouzbahman, Vicente Morales-Oyarvide, Kyu-Rae Kim, G P Nielsen, Esther Oliva. Lahey Hospital, Burlington, MA; Massachusetts General Hospital, Boston, MA; Toronto General Hospital, Toronto, ON, Canada; Dana Farber Cancer Institute, Boston, MA; University of Ulsan, Seoul, Korea. Background: Inflammatory myofibroblastic tumor (IMT) is rare in the uterus with only two small series reported.

Design: We evaluate clinical, morphologic, immunohistochemical, and molecular features of 13 uterine IMTs.

Results: Patients ranged from 8 to 78 (mean 45) years and tumors from 2.5 to 20 (mean 13) $\mathrm{cm}$. Extrauterine disease was noted in 4 . Compact growth ranged from 0-99\% (mean 35\%) with 10 having smooth muscle-like morphology, followed by storiform and fascicular (non-smooth muscle-like) (5 each), collagenous (4), nodular (3), and endometrial stromal-like and diffuse sheets (1 each). Mxyoid growth ranged from $1-100 \%$ (mean $65 \%$ ) with 12 having fascicular morphology, followed by nodular (10), storiform (8), pseudocysts (6), hypocellular (6), and smooth muscle-like (3). All had infiltrative borders and thin-walled ectatic vessels. Necrosis was seen in 8 and lymphovascular invasion in 2. All had a diffuse lymphoplasmacytic infiltrate that was lymphocyte-predominant in 5 . Nuclear atypia was mild in 5 , moderate in 5 , and severe in 3, with ganglion-like cells in 8 . Mitoses ranged from 0 to 41 (mean 8)/10 HPF. IHC and molecular results are shown below. Follow-up (11/13) ranged from 1 to 132 (mean 35) months with 5 alive and well, 2 alive with disease, and 4 dead of disease. On univariate analysis size $>7 \mathrm{~cm}$, moderate to severe atypia, and necrosis were associated with aggressive behavior.

\begin{tabular}{|l|l|l|l|l|l|l|}
\hline Case & CD10 & Desmin & Caldesmon & ALK IHC & ALK FISH & $\begin{array}{c}\text { Anchored } \\
\text { Multiplex Assay } \\
\text { (ALK, ROS1, } \\
\text { RET) }\end{array}$ \\
\hline 1 & + & + & + & + & + & Not Done (ND) \\
\hline 2 & + & + & + & - & + & - \\
\hline 3 & + & + & - & + & Failed (F) & TIMP3-ALK \\
\hline 4 & - & ND & - & - & F & ND \\
\hline 5 & + & - & - & - & + & - \\
\hline 6 & + & + & - & + & + & ND \\
\hline 7 & + & + & - & + & F & ND \\
\hline 8 & - & + & - & + & + & ND \\
\hline 9 & + & + & + & + & - & SEC31-ALK \\
\hline 10 & + & - & - & + & F & - \\
\hline 11 & + & - & ND & - & Pending (P) & ND \\
\hline 12 & - & + & - & + & P & ND \\
\hline 13 & ND & ND & ND & + & + & ND \\
\hline
\end{tabular}


Conclusions: $46 \%$ of IMTs were malignant with size $>7 \mathrm{~cm}$, moderate to severe atypia, and necrosis being predictive of aggressive behavior. These 3 features were seen in all malignant IMTs. IMTs often show histologic and immunohistochemical overlap with smooth muscle/stromal tumors; thus, ALK IHC and molecular studies are often indicated for correct diagnosis/management given the availability of targeted therapies for ALK rearranged tumors.

1110 Mismatch Repair Protein Expression in Endometrioid Carcinoma of the Ovary: Incidence and Clinicopathologic Associations in $\mathbf{7 7}$ Cases

Jennifer Bennett, Anna Pesci, Jason Badrinarain, Annacarolina Da Silva, Esther Oliva. Lahey Hospital, Burlington, MA; Ospedale Sacro Cuore-Don Calabria, Verona, Italy; Dana Farber Cancer Institute, Boston, MA; Massachusetts General Hospital, Boston, MA.

Background: The morphology of tumors deficient in mismatch repair (MMR) proteins has been well-described in endometrial and colorectal carcinomas, but few series have evaluated clinicopathologic associations in ovarian carcinomas.

Design: We evaluated a series of pure ovarian endometrioid carcinomas (OEC) for clinicopathologic features including age, size, stage, grade, precursor lesion (endometriosis, adenofibroma), peritumoral lymphocytes (PTL), intratumoral stromal inflammation (ISI), tumor infiltrating lymphocytes (TIL), mitoses, squamous metaplasia, clear cell change, mucinous differentiation, and sex cord-like elements. MMR status was determined by immunohistochemistry for MLH1, PMS2, MSH2, and MSH6.

Results: Loss of MMR expression was noted in 14\% of OECs and included MLH1/ PMS2 (5), MSH2/MSH6 (5), and PMS2 (1). Synchronous endometrial carcinomas from patients with MMR loss showed the same MMR immunoprofile (not performed in two). A comparison of clinicopathologic features in OECs with MMR loss versus all tumors is depicted below.

\begin{tabular}{|l|l|l|}
\hline \multicolumn{1}{|c|}{ Feature } & \multicolumn{1}{|c|}{ All OECs } & OECs with MMR Loss \\
\hline Age (years)* & $31-85(57)$ & $41-61(50)$ \\
\hline Size $(\mathrm{cm})$ & $0.3-30(13)$ & $0.7-18(9.5)$ \\
\hline Bilateral & $22 \%$ & $27 \%$ \\
\hline $\begin{array}{l}\text { Synchronous } \\
\text { Endometrial Tumor (all stage IA)* }\end{array}$ & $31 \%$ & $82 \%$ \\
\hline Stage & I:71\%, II: $14 \%$, III: $12 \%$, IV:3\% & I:82\%, II: $18 \%$ \\
\hline Grade & I:60\%, II:31\%, III:9\% & I:64\%, II:27\%, III:9\% \\
\hline Precursor & $68 \%$ & $64 \%$ \\
\hline PTLs & $3 \%$ & $9 \%$ \\
\hline ISI & $9 \%$ & $27 \%$ \\
\hline TILs* & $5-281(58)$ & $19-215(94)$ \\
\hline Mitoses & $1-77(16)$ & $6-46(18)$ \\
\hline Squamous Metaplasia & $64 \%$ & $73 \%$ \\
\hline Clear Cell Change & $34 \%$ & $55 \%$ \\
\hline Mucinous Differentiation & $23 \%$ & $36 \%$ \\
\hline Sex Cord-Like & $14 \%$ & $18 \%$ \\
\hline$*$ p $<0.05$ & & \\
\hline
\end{tabular}

Two patients with MSH2/MSH6 loss underwent molecular analysis; one had MSH2 germline mutation while the other had a MSH6 mutation. MLH1 promoter hypermethylation is pending for OECs with MLH1/PMS2 loss. Follow-up for all patients ranged from 1 to 307 (mean 101) months with $77 \%$ alive and well, 14\% dead of disease, $8 \%$ dead from other causes, and $1 \%$ alive with disease. All patients with MMR loss are alive and well (range 6-237 months, mean 104), but no statistically significant difference between survival and MMR status was noted.

Conclusions: We demonstrated loss of MMR expression in $14 \%$ of unselected OECs. A younger age at diagnosis, increased TILs $(>60)$, and a synchronous endometrial carcinoma were all predictive of MMR deficiency.

\section{KRAS or BRAF Mutation in Ovarian Low-Grade Serous} Carcinoma: An Analysis of 32 Chinese Patients

Rui Bi, Yan Xu, Ling Shan, Yaoxing Xiao, Xiaoyu Tu, Xiaoyan Zhou, Wentao Yang. Fudan University Shanghai Cancer Center, Shanghai, China.

Background: Several reports have demonstrated that KRAS and BRAF mutations occur at a frequency of $16 \%-54 \%$ and $2 \%-33 \%$, respectively, in ovarian low-grade serous carcinoma (LGSC). Evidences have shown that KRAS and BRAF are somatic mutations associated with LGSC. However, the mutation rate of KRAS and BRAF varied a lot in literature. Meanwhile, the prognostic significance of KRAS and BRAF mutations remains controversial.

Design: Codons 12 and 13 of exon 2 of KRAS and exon 15 of BRAF were analyzed using direct Sanger sequencing to identify mutations in 32 cases of LGSC. The relationships between mutations, overall survival (OS), disease-free survival (DFS) and clinicopathological characteristics were statistically analyzed.We further investigated the association between BRAF V600E mutations and immunohistochemistry using a monoclonal mouse antibody (VE1) that specifically detects mutated BRAF V600E protein in LGSC.

Results: KRAS and BRAF mutations were found in 9 cases $(9 / 32,28.13 \%)$ and 2 cases $(2 / 32,6.25 \%)$ respectively. The two mutations were mutually exclusive. No significant differences were identified in OS rates between patients with KRAS mutations (median OS, 30 months) and patients with wild-type KRAS genes (median OS, 22 months) $(\mathrm{P}=0.282)$. BRAF mutation status also had no significant differences in OS. BRAF
V600E protein was expressed in 3 cases. Compared with the mutation analysis, the sensitivity and specificity was $100 \%$ and $96.7 \%$ respectively. The concordance rate between protein expression and mutation was $96.88 \%$.

Conclusions: The present study indicated low frequency of KRAS and BRAF mutations in Chinese LGSC patients. More studies about other abnormalities in LGSCs should be carried out. Neither KRAS nor BRAF mutation status is a prognostic factor in LGSC. BRAF mutation specific protein expression and mutation had a high rate of concordance and could therefore be served as a screening method for mutation analysis.

1112 Sentinel Lymph Node Ultrastaging as a Supplement for Endometrial Cancer Intraoperative Frozen Section Deficiencies

Morgan Blakely, Yuxin Liu, Jamal Rahaman, Monica Prasad-Hayes, Navya Nair, Tamara Kalir. Mount Sinai Hospital Icahn School of Medicine, New York, NY.

Background: Due to inherent intraoperative frozen section (IFS) limitations, approx. $15 \%$ of endometrial cancer (EC) patients are under- or over-treated with lymph node dissection upon initial surgery. Sentinel lymph node (SLN) ultrastaging is an emerging strategy to detect occult nodal disease. We hypothesize that SLNs will provide useful prognostic information for cases misclassified by IFS.

Design: EC with hysterectomy, IFS, SLN were included. IFS/final diagnoses, total positive SLN and pelvic LN were recorded.

Results: 50 cases ( 37 low-grade, 13 high-grade) included. Patient \& tumor factors shown in Table 1. During IFS, 18 cases were deemed "high risk" for nodal metastases whereas 32 "low risk" based on tumor grade and/or depth of myometrial invasion. Compared to final diagnosis, IFS misclassified the risk in 10 cases and would have resulted in 6 inadequately staged and 4 over-treated with LND. SLNs, obtained in 47 cases, were positive in two (4.2\%): $0.1 \mathrm{~mm}$ metastases in a patient estimated as low-risk on IFS (Fig 1C,D); $1 \mathrm{~mm}$ metastases in a high risk patient (Fig 1A,B). The remaining 45 patients, including 9 cases misclassified on IFS, revealed negative SLN and pelvic LNs.

\begin{tabular}{|l|c|}
\hline \multicolumn{1}{|c|}{ Factors } & Case (\%) \\
\hline Age, median (range) & $65(45-84)$ \\
\hline Tumor size, median (range) & $3 \mathrm{~cm}(0-6)$ \\
\hline Tumor type & \\
\hline Endometrioid & $43(85 \%)$ \\
\hline Serous & $5(11 \%)$ \\
\hline Carcinosarcoma & $2(4 \%)$ \\
\hline FIGO Grade & \\
\hline 1 & $26(54 \%)$ \\
\hline 2 & $11(20 \%)$ \\
\hline 3 & $13(26 \%)$ \\
\hline FIGO Stage & \\
\hline I & $46(92 \%)$ \\
\hline II & $2(4 \%)$ \\
\hline III & $2(4 \%)$ \\
\hline Depth of Myometrial Invasion & \\
\hline less than 50\% & $43(83 \%)$ \\
\hline $50 \%$ or more & $7(17 \%)$ \\
\hline Lymphovascular invasion & \\
\hline Present & $10(20 \%)$ \\
\hline Absent & $40(80 \%)$ \\
\hline Lymph Node & \\
\hline SLN, mean (range) & $4.9(1-12)$ \\
\hline Non-SLN, mean (range) & $8.9(1-22)$ \\
\hline
\end{tabular}

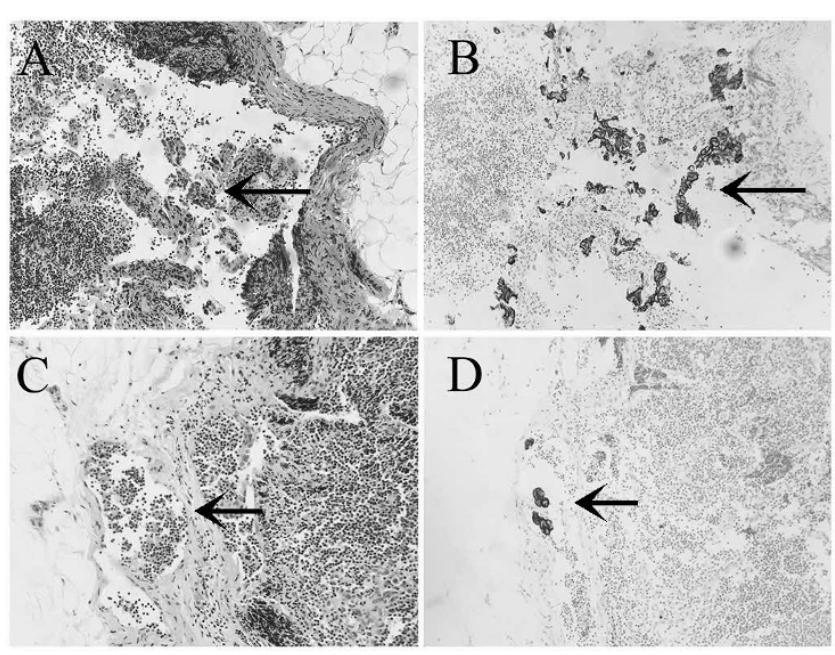

Conclusions: IFS over- or underestimated nodal disease risk in $20 \%$ of our cases. SLN ultrastaging detected micrometastasis otherwise would have been missed. Our results support SLN as an effective supplement to provide important prognostic information on nodal status, in particular, to avoid inadequate staging during initial surgery. 
1113 Molecular Classification of Grade 3 Endometrioid Endometrial Cancers Identifies Distinct Prognostic Subgroups

Tjalling Bosse, Remi A Nout, Jessica N McAlpine, Melissa McConechy, Heidi Britton, Raji Ganesan, Jane C Steele, Beth T Harrison, Esther Oliva, Xavier Matias-Guiu, Blake Gilks, Robert Soslow. Leiden University Medical Center, Leiden, Netherlands, University of British Columbia, Vancouver, Canada; University of British Columbia and BC Cancer Agency, Vancouver, Canada; Birmingham Women's HS Foundation Trust, Birmingham, United Kingdom; Massachussets General Hospital, Boston, MA; Hospital U Arnau de Vilanova and Hospital U de Bellvitge, Lleida, Spain; Memorial Sloan Kettering Cancer Center, New York, NY; McGill University, Montreal, Canada. Background: This study aimed to investigate whether molecular classification can be used to refine prognosis in grade 3 endometrioid endometrial carcinomas (EEC). Design: Grade 3 EEC's were subclassified into four subgroups: 5 53-abnormal based on mutant-like immunostaining (p53-abn), MMR-deficient based on loss of mismatch repair protein expression (MMRd), presence of POLE exonuclease domain hotspot mutation (POLE-mutant) and no specific molecular profile (NSMP) in which none of these aberrations were present. Tumors with p53 aberration lacking POLE mutation but with aberrant MMR were categorized as p53-abn. Overall (OS), and disease free survival (DFS) rates were compared using Kaplan Meier method (Log Rank test) and univariable and multivariable Cox proportional hazard models.

Results: In total 358 patients were included with a median age of 66 years (range 33-96) and FIGO stage (2009): IA 164 (45.8\%), IB $112(31.3 \%)$, II-IV 78 (21.8\%). There were 47 (13.1\%) POLE mutant, 71 (19.8\%) p53-abn, 102 (28.5\%) NSMP and $138(38.5 \%)$ MMRd tumors. Median follow-up of patients alive was 5.98 years (range 0.3-17). Compared to NSMP, patients with POLE mutant EC (OS: Hazard Ratio [HR] 0.28, [95\%CI: 0.14-0.57], $\mathrm{p}=0.0005$; DFS: HR 0.22 [0.09-0.51], $\mathrm{p}=0.0004)$ or MMRd (OS HR 0.66 [0.45-0.98], $\mathrm{p}=0.037$; DFS: HR 0.51 [0.33-0.80], $\mathrm{p}=0.003$ ) have a significantly better prognosis, while that of p53-abn EC did not differ significantly. Estimated 5-year OS rates were: POLE mutant $91 \%$, MMRd $79 \%$, NSMP $65 \%$, and p 53 -abn $55 \%$ (Log Rank $\mathrm{p}=0.0001)$; and 5 -year DFS rates $91 \%, 77 \%, 60 \%, 50 \%(\mathrm{p}=0.000003)$. In a multivariable Cox model that included age and FIGO stage, both POLE mutant and MMRd status remained independent prognostic factors for better OS and DFS.

Conclusions: Molecular classification of Grade 3 EECs reveals that these tumours are a mixture of molecular subtypes, rather than a homogeneous group.

The addition of molecular markers identifies prognostic subgroups with potential therapeutic implications.

1114 Does Universal Tissue Testing Provide Universal Answers? Clinical Challenges Associated with Tumor Screening for Lynch Syndrome Associated Endometrial Cancer

Amanda Bruegl, Molly Daniels, Russell R Broaddus. Oregon Health and Science University, Portland, OR; MDACC, Houston, TX.

Background: Tumor testing for Lynch Syndrome is a component of the diagnostic work-up of endometrial cancer patients. Such screening has important implications in the cancer prevention care for the index patient and for her relatives. The purpose of this study was to identify prospectively the barriers to universal screening based on a tissue testing approach (microsatellite instability (MSI) analysis, immunohistochemistry (IHC) for DNA mismatch repair (MMR) proteins, and MLHI methylation analysis). Such barriers may preclude definitive characterization of a patient's tumor as sporadic or hereditary.

Design: Endometrial carcinoma patients $(\mathrm{n}=213)$ prospectively underwent MSI and IHC testing for MMR proteins. Patients with low (MSI-L) or high (MSI-H) levels of tumor microsatellite instability or IHC loss of MLH1 (and absent MLH1 methylation), MSH2, MSH6, or PMS2 were referred to a genetic counselor for consideration of germline testing.

Results: $71.9 \%$ of tested tumors were MSS, $25.1 \%$ MSI-H, and 3\% MSI-L. $22.8 \%$ of patients had tumors with loss of MLH1/PMS2, 1.0\% loss of MSH2/MSH6, 1.5\% loss of MSH6, and $1.5 \%$ loss of PMS2. Six discordances (3.1\% of tested cases) between IHC and MSI were identified. Half of these exhibited heterogeneous IHC loss of MLH1/ PMS2 and were MSS. Of the remaining cases, one was MSS with IHC loss of MSH6, one was MSS with IHC loss of MLH1/PMS2 and absent MLH1 methylation, and one was MSI-H with intact MMR protein expression. Four patients had MSI-L tumors with intact MMR IHC; the clinical significance of MSI-L in endometrial cancer is unclear so each was offered genetic counseling. 15/213 (7\%) of patients had tissue testing results suggestive of Lynch Syndrome, but germline mutations were only detected in 7 patients. Conclusions: We encountered barriers to universal screening in $13.6 \%$ of screened patients (29/213) that precluded designation of a tumor as sporadic or hereditary. The number of instances in each of the pre-analytic (patient or insurance declining screening), analytic (insufficient tissue, ambiguous results, discordance between IHC and MSI), and post-analytic (patient or insurance declines genetic counseling/testing, informative tumor testing with negative germline testing, or germline testing showing a variant of unknown significance) categories is small, but it is clear that problems can arise at numerous steps along the path to universal testing.

\section{HPV E6/E7RNA In Situ Hybridization Signal Patterns Utility for} Cervical Lesion Grading

Britni RE Bryant, Mark Evans, Maureen Harmon, Scott Anderson, Alexandra Kalof. University of Vermont College of Medicine / Medical Center, Burlington, VT. Background: Cervical lesion grading is controversial yet critical because of the potential for patient under- or over-treatment. Previously we reported preliminary data that highrisk HPV E6/E7 RNA in situ hybridization (ISH) signal patterns (graded as 1, 2 or 3) show direct correlations with routine diagnoses of cervical intraepithelial neoplasia (CIN) grades 1, 2 and 3 respectively. In this study, ISH staining was compared against consensus H\&E CIN diagnoses and $\mathrm{p} 16^{\text {NK4a }} \mathrm{IHC}$ staining.
Design: Cervical specimens from 114 patients were independently CIN graded by three pathologists. p16 IHC was two-tier rated according to epithelial thickness staining. RNA ISH signals patterns were scored: 0 (negative/sporadic), 1 (upper layer diffuse), 2 (2/3 lower layer dot signals plus superficial diffuse), 3 (full thickness dot signals and absent or sporadic superficial diffuse).

Results: Consensus diagnoses relative to ISH and p16 staining patterns are shown in Table 1. Unanimous H\&E consensus was better for CIN2 than CIN3 but not significantly so $(\mathrm{p}=0.078)$.

\begin{tabular}{|r|c|c|c|c|c|}
\hline & $\begin{array}{c}<\mathrm{CIN} \\
(\mathrm{n}=5)\end{array}$ & $\begin{array}{c}\mathrm{CIN} 1 \\
(\mathrm{n}=27)\end{array}$ & $\begin{array}{c}\mathrm{CIN} 2 \\
(\mathrm{n}=44)\end{array}$ & $\begin{array}{c}\mathrm{CIN} 3 \\
(\mathrm{n}=37)\end{array}$ & $\begin{array}{c}\text { No Consensus } \\
(\mathrm{n}=1)\end{array}$ \\
\hline & $1(20 \%)$ & $17(63.0 \%)$ & $29(65.9 \%)$ & $17(45.9 \%)$ & $0(0 \%)$ \\
\hline ISH0 & $4(80 \%)$ & $15(55.6 \%)$ & $7(15.9 \%)$ & $2(5.4 \%)$ & $1(100 \%)$ \\
\hline ISH1 & $1(20 \%)$ & $6(22.2 \%)$ & $3(6.8 \%)$ & $0(0 \%)$ & $0(0 \%)$ \\
\hline ISH2 & $0(0 \%)$ & $4(14.8 \%)$ & $22(50.0 \%)$ & $0(0 \%)$ & $0(0 \%)$ \\
\hline ISH3 & $0(0 \%)$ & $2(7.4 \%)$ & $12(27.3 \%)$ & $35(94.6 \%)$ & $0(0 \%)$ \\
\hline $\mathrm{p} 16 \leq \mathrm{LGSIL}$ & $5(100 \%)$ & $21(77.8 \%)$ & $8(18.2 \%)$ & $0(0 \%)$ & $0(0 \%)$ \\
\hline $\mathrm{p} 16-$ HGSIL & $0(0 \%)$ & $6(22.2 \%)$ & $36(81.8 \%)$ & $37(100 \%)$ & $1(100 \%)$ \\
\hline
\end{tabular}

Table 2. Test sensitivities, specificities and predictive values for consensus CIN grade.

\begin{tabular}{|r|c|c|c|c|}
\hline \multicolumn{1}{|l|}{ Test } & Sensitivity & Specificity & $\begin{array}{l}\text { Positive } \\
\text { Predictive } \\
\text { Value }\end{array}$ & $\begin{array}{l}\text { Negative } \\
\text { Predictive } \\
\text { Value }\end{array}$ \\
\hline ISH0/1 for $\leq \mathrm{CIN} 1$ & $66.7 \%$ & $92.0 \%$ & $81.3 \%$ & $84.2 \%$ \\
\hline $\mathrm{ISH} 1$ for $\mathrm{CIN} 1$ & $60.0 \%$ & $79.8 \%$ & $22.2 \%$ & $95.4 \%$ \\
\hline $\mathrm{ISH} 2$ for $\mathrm{CIN} 2$ & $84.6 \%$ & $75.0 \%$ & $50.0 \%$ & $94.3 \%$ \\
\hline $\mathrm{ISH} 3$ for $\mathrm{CIN} 3$ & $71.4 \%$ & $96.9 \%$ & $94.6 \%$ & $81.8 \%$ \\
\hline $\mathrm{ISH} 2 / 3$ for $\mathrm{CIN} 2 / 3$ & $92.0 \%$ & $69.2 \%$ & $85.2 \%$ & $64.5 \%$ \\
\hline $\mathrm{p} 16$ for $\mathrm{CIN} 2 / 3$ & $91.3 \%$ & $76.5 \%$ & $90.1 \%$ & $78.8 \%$ \\
\hline
\end{tabular}

Conclusions: ISH signal patterns are not simple correlates of consensus H\&E diagnoses; however, the data are supportive of ISH as a judicious diagnostic test: ISH0/1 staining to confirm/favor CIN1/LGSIL, ISH2 to support CIN2/HGSIL, and ISH3 for CIN3/ HGSIL. Further studies are required to understand the significance of mismatched ISH staining and $\mathrm{H} \& \mathrm{E}$ diagnoses.

1116 Outcome

Tatiana N Buhtoiarova, Jennifer Zeng, Khush Mittal. UH Parma, Parma, OH; NYU Langone Medical Center, New York, NY.

Background: Placental macrocalcifications are often found in term pregnancy and considered as a physiological aging process. The possible association of excessive placental macrocalcifications and adverse pregnancy outcome is unclear.

Design: A total of 272 placentas were studied from the following clinical and histopathological diagnosis: pre-term birth, post-term birth, gestational diabetes mellitus (GDM), placental abruption (PA), pre-eclampsia (PE), intrauterine fetal demise (IUFD), intrauterine growth restriction (IUGR), acute chorioamnionitis (AC), fetal anomalies (FA), chronic villitis (CV), non-reassuring fetal heart rate (NRFHR), pregnancy-induced hypertension (PIH) and placental infarct (PI). The control group includes 17 placentas from normal spontaneous vaginal delivery (NSVD). The hematoxylin and eosin (H\&E) slides were retrieved from pathology department database at our institution between January 2014 and December 2015 and were evaluated by the authors for the presence of excessive placental macrocalcifications (EPMAC). The correspondent maternal clinical history and fetal outcome were obtained from an electronic medical record and reviewed. The results were correlated with clinicopathological features and fetal outcome.

Results: A total of 272 placentas were studied. EPMAC were observed in categories of placental infarct $(25 \%)$, non-reassuring fetal heart rate $(22.2 \%)$, fetal anomalies $(10 \%)$, and post-date pregnancies $(9.5 \%)$.
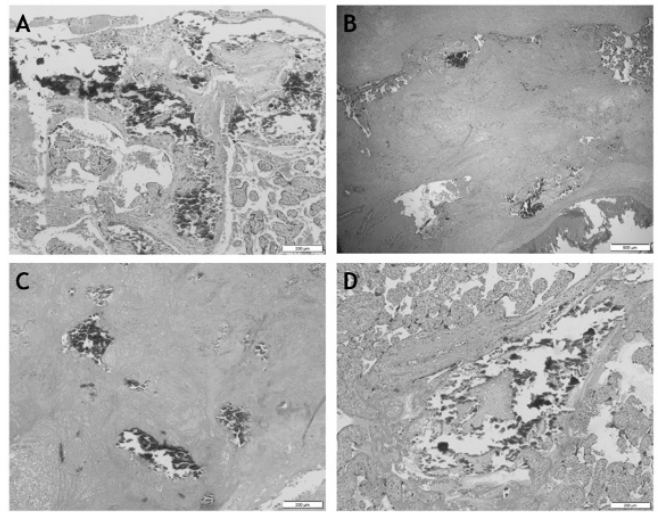

Figure 1

A. Calcifications in maternal plate ( $\mathrm{H} \& \mathrm{E}$, original magnification $\times 100)$

B. Calcifications in fetal plate associated with fibrinoid deposition (H\&E, original magnification $\times 40$ )

C. Calcitions

C. Calcifications in area or inter

D. Calcifications in stem villus(H\&E, original magnification $\times 100$ )

In the categories of preterm birth, gestational diabetes mellitus and intrauterine growth restriction, EPMAC were found in approximately $5 \%$ of cases. The remaining categories demonstrate an absence of EPMAC. 
Conclusions: Calcification of the placenta is a common finding and observed in all groups. EPMAC is most commonly observed in placentas with infarct $(25 \%)$ and nonreassuring fetal heart rate $(22.2 \%)$. EPMAC are indicative of a high-risk pregnancies. Additional studies are warranted for further investigation of the clinical significance of excessive placental macrocalcifications.

1117 IFITM1 Outperforms CD10 in Differentiating Low Grade Endometrial Stromal Sarcomas from Smooth Muscle Neoplasms

Aurelia Busca, Carlos Parra-Herran, Previn Gulavita, Shahid Islam. University of Ottawa and The Ottawa Hospital, Ottawa, ON, Canada; University of Toronto and Sunnybrook Health Sciences Centre, Toronto, ON, Canada; University of Ottawa and Montfort Hospital, Ottawa, ON, Canada.

Background: Distinguishing between uterine neoplasms of smooth muscle and endometrial stromal origin is a frequent diagnostic challenge. We investigated the staining pattern of interferon-induced transmembrane protein-1 (IFITM1), a novel endometrial stromal marker, in endometrial and smooth muscle uterine neoplasms and compared it to CD10 in its ability to differentiate between these two groups.

Design: Immunohistochemistry for IFITM1 and CD10 was performed in 10 cases of smooth muscle neoplasms ( 5 cases leiomyoma, 5 cases leiomyosarcoma), 7 cases of endometrial stromal sarcoma ( 5 cases low grade and 2 cases high grade) and 13 cases of carcinosarcoma. Staining was scored in terms of intensity and distribution ( $0=a b s e n t$, $1=$ weak $/$ less than $50 \%, 2=$ moderate $/ 50-75 \%, 3=$ strong $/$ more than $75 \%$ ). A total score was obtained by adding intensity and distribution scores and classified as positive (score 3-6) or negative (score 0-2).

Results: IFITM1 was positive in $5 / 5(100 \%)$ low grade endometrial stromal sarcomas, $0 / 10$ smooth muscle tumors (leiomyomas and leiomyosarcomas) and 11/13 carcinosarcomas $(84.6 \%)$. The two cases of high grade endometrial stromal sarcoma were IFITM1 negative. While both IFITM1 and CD10 had $100 \%$ sensitivity in distinguishing low grade endometrial stromal sarcomas from smooth muscle neoplasms, IFITM1 $(100 \%)$ had higher specificity compared to CD10 $(70 \%)$.

Conclusions: In this study IFITM1 appears to be a more specific marker of endometria stromal differentiation compared to CD10 in distinguishing low grade endometrial stromal sarcomas from smooth muscle neoplasms. Thus, IFITM1 may be a valuable tool as part of an immunohistochemical evaluation panel in this diagnostic scenario.

\section{Utility of CK7 and p16 in Classification of a Rare Variant of} Squamous Intraepithelial Lesion of the Cervix

Katelynn Campbell, Charles M Quick, Mayumi Nakagawa, Susanne Jeffus. University of Arkansas for Medical Sciences, Little Rock, AR.

Background: Maturing atypical dysplasia (MAD) represents a rare, distinct subset of squamous intraepithelial lesions (SIL) of the cervix. MADSIL is characterized by markedly enlarged and bizarre nuclei with nuclear size variability of at least 3:1. Chromatin appears smudged with hyper- and polychromasia. Abundant cytoplasm results in a low N/C ratio. Atypical mitotic figures can be seen.

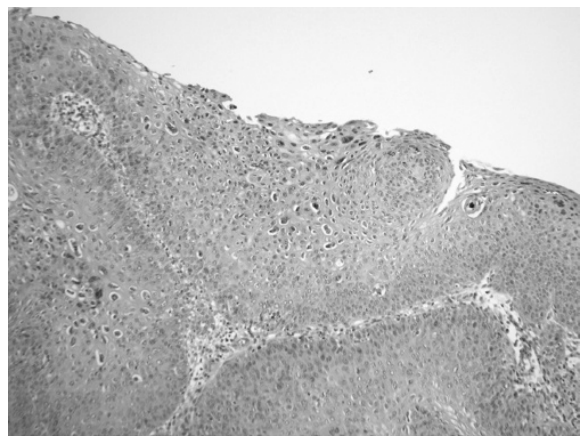

P16 staining subcategorizes difficult cases as LSIL or HSIL/CIN2; however, p16 is positive in a subset of LSIL and fails to risk stratify this cohort. CK7 is a biomarker for SIL arising from the squamocolumnar junction (SCJ), demonstrating positivity in HSIL and LSIL "progressors". This study examines the utility of p16 and CK7 with correlation to HPV-PCR and clinical follow-up to determine whether MADSIL should be classified as LSIL or HSIL.

Design: 738 consecutive cervical biopsies (2009-2011) were reviewed by 2 gynecologic pathologists and 1 resident for consensus diagnosis of MADSIL. HPV-PCR, Ck7 (Dako OV-TL 12/30; dilution 1:400) and p16 staining was performed on most representative MADSILs ( $\mathrm{n}=30$ ). $\mathrm{P} 16$ was scored as positive (block positivity) or negative. $\mathrm{Ck} 7 \mathrm{was}$ interpreted as positive (gradient/top down or full thickness staining in at least 5-6 contiguous cells) or negative (patchy or no staining). PCR analysis for low and high risk HPV types was performed. Clinical follow up data was collected by chart review. Results: $45(6 \%)$ MADSILs were identified. The original diagnosis for the 30 selected MADSILs was LSIL (37\%) and HSIL/CIN2 (63\%). 53\% (16/30) showed atypical mitotic figures. Staining was successful in 29/30. 26/29 (90\%) MADSILs were p16 positive. CK7 was positive in $66 \%$ (full thickness: $13 / 19$, gradient: 6/19). Co-expression of 16 and CK7 was seen in 17/29 (59\%); all CK7 positive cases were also positive for p16. HPV subtyping was successful in $47 \%$ of MADSILs; all were hrHPV positive [16 (3), 18 (3), 26, 33, 35 (3), 39, 51 (2), 59, 66]. Follow up showed HSIL on LEEP in 2/2 cases (case 1: p16, CK7 co-expression, case 2: p16 positive/CK7 negative).

Conclusions: Given the predominant positivity for $\mathrm{p} 16$, frequent $\mathrm{p} 16 / \mathrm{Ck} 7 \mathrm{co-expression}$ and exclusive association with hrHPV, MADSIL should be classified as HSIL (CIN 2).
1119 Clinical Outcomes in MLH1-Methylated Endometria Carcinomas

Sydney Card, Koah Vierkoetter, Teri Longacre. Stanford University Medical Center, Stanford, CA

Background: The majority of sporadic endometrial carcinomas (EMCs) that demonstrate microsatellite instability/mismatch repair deficiency arise as a consequence of $M L H 1$ promoter methylation. We obtained follow-up clinical and pathologic data on $\mathrm{MLH1}$-methylated EMCs to determine if clinical outcomes differed from all cases of EMC.

Design: 106 cases of EMC (2011-2016) with mismatch repair protein deficiency and MLH1 promoter methylation were reviewed to determine stage at diagnosis, adjuvant therapy status, and stage and clinical outcome at the time of the last clinical visit. Median follow-up time was 23 months (range: 1 - 96 months).

Results: 69 patients were alive with no evidence of disease; of these, 36 received no adjuvant therapy after initial diagnosis or surgery. 32 received at least one form of adjuvant therapy: 18 were treated with radiotherapy alone (external beam, brachytherapy, or both); 11 were treated with combination chemotherapy and radiotherapy, and 3 were treated with chemotherapy alone. 3 patients died of unrelated causes, without recurrence or progression of their EMC.

\begin{tabular}{|l|l|}
\hline Outcome & Number of Cases \\
\hline Alive with no evidence of disease & $68(64.2 \%)$ \\
\hline Alive with disease & $23(21.7 \%)$ \\
\hline Died of disease (EMC) & $8(7.5 \%)$ \\
\hline Died of other causes & $3(2.8 \%)$ \\
\hline No recent data available & $4(3.8 \%)$ \\
\hline
\end{tabular}

23 patients were alive with disease, including 8 patients with local (vaginal or pelvic side wall) recurrence and 16 patients who developed lymph node or distant metastases not present at the time of diagnosis.

8 patients died due to EMC. In this group, all but one presented with high-stage (FIGO III/IV) disease. Median and mean survival times for these patients were 10.5 and 17.6 months, respectively. 3 patients received adjuvant chemotherapy alone, 4 received adjuvant chemotherapy and radiotherapy, and one received neoadjuvant chemotherapy followed by adjuvant chemotherapy and radiotherapy.

Conclusions: When compared with SEER and FIGO data sets for all EMCs, EMCs with $M L H 1$ promoter methylation do not follow a substantially different clinical course. As with all other EMCs, pathologic stage remains the most reliable prognostic factor.

\section{Clinicopathologic Features of $M L H 1$-Methylated Endometria} Carcinomas

Sydney Card, Koah Vierkoetter, Teri Longacre. Stanford University Medical Center, Stanford, CA.

Background: The majority of sporadic endometrial carcinomas (EMCs) that demonstrate mismatch repair deficiency arise as a consequence of $M L H 1$ promoter methylation. We obtained data on clinicopathologic features to determine trends in this subset of EMCs.

Design: 106 cases of EMCs with $M L H I$ promoter methylation were assessed for clinical (age, presentation, body mass index [BMI], hormone replacement therapy [HRT]) and pathologic (tumor morphology, grade, stage, LVI, biomarkers) features. Age at diagnosis ranged from $40-88 \mathrm{y}$ (median: $65 \mathrm{y}$ ). A previously proposed cut-off for tumor-infiltrating lymphocytes (TILs) $(\geq 42 / 10 \mathrm{HPF}=$ present $;<42 / 10 \mathrm{HPF}=$ absent $)$ was used.

Results: $M L H 1$-methylated EMCs showed an association with increased BMI (median: $\left.29.6 \mathrm{~kg} / \mathrm{m}^{2}\right)$ and peri-/postmenopausal bleeding (PMB). The majority of patients received no HRT prior to their cancer diagnosis.

\begin{tabular}{|l|l|l|}
\hline Clinical Feature & Category & $\mathrm{N}(\%)$ \\
\hline \multirow{5}{*}{ BMI } & Underweight & $3(2.9 \%)$ \\
\cline { 2 - 3 } & Normal & $21(20.0 \%)$ \\
\cline { 2 - 3 } & Overweight & $30(28.9 \%)$ \\
\cline { 2 - 3 } & Obese & $51(48.6 \%)$ \\
\cline { 2 - 3 } & N/A & $1(1.0 \%)$ \\
\hline \multirow{5}{*}{ Presentation } & Peri-/postmenopausal bleeding & $79(74.5 \%)$ \\
\cline { 2 - 3 } & Abnormal uterine (premenopausal) bleeding & $11(10.4 \%)$ \\
\cline { 2 - 3 } & N/A & $10(9.4 \%)$ \\
\cline { 2 - 3 } & Other & $4(3.8 \%)$ \\
\cline { 2 - 3 } & Abnormal Pap test & $2(1.9 \%)$ \\
\hline \multirow{3}{*}{ Previous HRT } & No & $98(93.3 \%)$ \\
\cline { 2 - 3 } & Yes & $7(6.7 \%)$ \\
\hline
\end{tabular}

MLH1-methylated EMCs were associated with endometrioid type, FIGO grade I or II, low FIGO stage (I or II) at diagnosis, no LVI, and ER and PR expression. All cases with p53 stains showed a wildtype pattern. Lower uterine segment (LUS) tumors were a distinct minority. Most cases demonstrated TILs, with TIL-positive cases containing an average of 117 lymphocytes/10 HPF. 


\begin{tabular}{|c|c|c|}
\hline Pathologic Feature & Category & $\mathrm{N}(\%)$ \\
\hline \multirow{4}{*}{ Tumor Type } & Endometrioid & $99(93.4 \%)$ \\
\hline & Mucinous & $1(0.9 \%)$ \\
\hline & Dedifferentiated & $5(4.7 \%)$ \\
\hline & Undifferentiated & $1(0.9 \%)$ \\
\hline \multirow{4}{*}{ Main Tumor Site } & Corpus/fundus & $76(71.0 \%)$ \\
\hline & LUS & $5(4.7 \%)$ \\
\hline & Both & $15(14.0 \%)$ \\
\hline & N/A & $11(10.3 \%)$ \\
\hline \multirow{4}{*}{ FIGO Grade } & $\mathrm{I}$ & $44(41.1 \%)$ \\
\hline & II & $39(36.4 \%)$ \\
\hline & III & $15(14.0 \%)$ \\
\hline & N/A & $9(8.4 \%)$ \\
\hline \multirow{4}{*}{ FIGO Stage at Diagnosis } & I & $64(63.4 \%)$ \\
\hline & II & $11(10.9 \%)$ \\
\hline & III & $22(21.8 \%)$ \\
\hline & IV & $4(4.0 \%)$ \\
\hline \multirow{2}{*}{ LVI } & Present & $32(34.4 \%)$ \\
\hline & Not identified & $61(65.6 \%)$ \\
\hline \multirow{2}{*}{ TILs } & Present & $38(73.1 \%)$ \\
\hline & Absent & $16(26.9 \%)$ \\
\hline
\end{tabular}

underwent NACT with interval debulking surgery and had fallopian tubes processed by the SEE-FIM protocol. p53 and Ki67 staining was used to confirm diagnoses if morphologic findings were suspicious but not definitive. Chemotherapy-induced cytologic alterations, tubal mucosal denudation and granulation tissue were recorded. Primary site of tumor origin was retrospectively classified using ICCR criteria.

Results: Residual STIC was present in $41 / 135(30 \%)$ cases, 31 of which also had stromal submucosal invasion. Four cases (3\%) of invasive HGSC obscured the overlying mucosa. Submucosal HGSC without STIC was seen in 16(12\%); the overlying mucosa was either benign or denuded. Serosal HGSC without mucosal/submucosal tumor was seen in $41(30 \%)$. Tubes were not identified in $2(1 \%)$ cases, presumed to be replaced by residual HGSC. There was no residual tubal tumor in $31(15 \%)$. Chemotherapy-induced alterations were seen in $41 \%$, in some cases obscuring distinction between benign mucosa versus residual STIC, mainly due to limited nuclear atypia and absent/rare mitoses. Mucosal granulation tissue was in $20 \%$. Primary tumor origin was classified as tubal in $33 \%$, using presence of STIC/mucosal HGSC alone; $45 \%$ if also counting submucosal HGSC without STIC; or $47 \%$ if also counting tubes with presumed replacement by tumor.

Conclusions: NACT for advanced staged pelvic HGSC may complicate the evaluation for residual STIC and application of ICCR criteria for assigning primary origin of tumor. Mucosal denudation and granulation tissue may represent STIC with complete response to NACT while treatment-induced cytologic alterations may make detection of residual STIC difficult.

1123 PAX8 Expression Occurs in the Setting of BAP1 Loss in Malignant Peritoneal Mesothelioma

David B Chapel, Stephanie M McGregor, Aliya N Husain, Thomas Krausz. University of Chicago, Chicago, IL; University of Wisconsin-Madison, Madison, WI.

Background: Discrimination between malignant peritoneal mesothelioma (MPM) and serous carcinoma (SC) involving the peritoneum remains a diagnostic challenge, particularly in small biopsy and cytology specimens. In making this distinction, PAX8 expression by immunohistochemistry (IHC) has been regarded as a specific marker for SC. Additionally, discrimination between MPM and benign peritoneal mesothelia lesions (BPMLs) may be difficult. BAP1 loss has been reported to be specific for MPM, as BAP1 loss is reportedly very rare in SC and in BPMLs.

Design: All in-house cases of MPM diagnosed at our institution between 10/1/2003 and $6 / 30 / 2016(n=25)$ were subjected to IHC for PAX8 and BAP1. BPMLs were used as controls ( $\mathrm{n}=14 ; 10$ well-differentiated papillary mesotheliomas, 3 benign multicystic peritoneal mesotheliomas, and 1 benign mesothelial inclusion cyst). Complete absence of nuclear staining defined BAP1 loss. PAX8 was considered positive if nuclear staining was present in $\geq 30 \%$ of mesothelial cells. Statistical analysis was performed with Fisher's exact test.

Results: Four MPMs were PAX8 positive $(4 / 25,16 \%)$, while two BPMLs were PAX8 positive $(2 / 14,14 \%)$. Sixteen MPMs exhibited BAP1 loss $(16 / 25,64 \%)$, while no BPMLs exhibited BAP1 loss $(0 / 14$; see Table 1$)$. In peritoneal mesothelial lesions, BAP1 loss was significantly correlated with malignancy $(\mathrm{p}<0.0005)$, with sensitivity of $64 \%$ and specificity of $100 \%$ for diagnosis of MPM. All four PAX8-positive MPMs exhibited BAP1 loss.

\begin{tabular}{|l|c|c|c|c|}
\hline & \multicolumn{2}{|c|}{$\begin{array}{c}\text { PAX8 Expression } \\
{[\mathrm{n}(\%)]}\end{array}$} & \multicolumn{2}{c|}{$\begin{array}{c}\text { BAP1 Expression } \\
{[\mathrm{n}(\%)]}\end{array}$} \\
\hline & Positive & Negative & Retained & Lost \\
\hline Malignant peritoneal mesothelioma & $4(16)$ & $21(84)$ & $9(36)$ & $16(64)$ \\
\hline $\begin{array}{l}\text { Well-differentiated papillary } \\
\text { mesothelioma }\end{array}$ & $1(10)$ & $9(90)$ & 10 & 0 \\
\hline $\begin{array}{l}\text { Benign multicystic papillary } \\
\text { mesothelioma }\end{array}$ & 0 & 3 & 3 & 0 \\
\hline Mesothelial inclusion cyst & 1 & 0 & 1 & 0 \\
\hline
\end{tabular}

Conclusions: Though routinely used as evidence of Mullerian origin, PAX8 expression is not uncommon in mesothelial proliferations, whether benign or malignant. Accordingly, use of PAX8 IHC alone to distinguish MPM from SC involving the peritoneum should be undertaken with caution. Interestingly, in this study all PAX8positive MPMs also exhibited BAP1 loss. As the finding of BAP1 loss argues against $\mathrm{SC}$, these data suggest that using PAX8 and BAP1 IHC in combination may prevent diagnostic missteps when distinguishing SC from MPM. This study also confirms BAP1 loss as a highly specific marker for distinguishing MPM from BPMLs.

112 Teratomas

Gregory Charville, Teri Longacre, Hannes Vogel, Thomas M Ulbright, Chia-Sui Kao. Stanford University School of Medicine, Stanford, CA; Indiana University School of Medicine, Indianapolis, IN.

Background: It is important to distinguish between mature and immature teratoma in the ovary given the latter has a higher risk of malignant transformation and carries a worse prognosis, especially those with higher grade, which may require chemotherapy. The diagnosis of immaturity has traditionally relied on the recognition of primitive neuroepithelium on morphology, which sometimes may be subjective. We aimed to identify helpful immunohistochemical stains to aid in the objective identification of immature teratomas.

Design: Twenty-three cases of immature ovarian teratoma, along with samples of non-neoplastic cerebral cortex ( 5 adult and 5 neonatal) and cerebellum ( 5 adult and 5 neonatal), were selected. Immunohistochemical stains for Ki67, cyclin D1, OCT3/4, and NeuN were performed, and expression of each stain was recorded for both immature and mature elements within the immature teratoma as well as the non-neoplastic brain tissue. 
Results: Proliferative index was increased ( $>25 \%$ staining) in the immature neuroepithelium in all cases $(23 / 23)$ as assessed by either Ki67 or cyclin D1 expression. Cyclin D1 was a more sensitive marker of increased proliferation than Ki67, identifying $90.9 \%$ of immature teratomas (versus $65.2 \%$ for Ki67). The average proliferative index was significantly increased $(P<0.05)$ in immature versus mature neural tissue as assessed by Ki67 (36 $\pm 6 \%$ vs. $2.1 \pm 0.6 \%$ ) or cyclin D1 ( $65 \pm 6 \%$ vs. $5 \pm 1 \%)$. OCT3/4 expression was only present in $6 / 23$ cases, corresponding to embryonal carcinoma; immature neuroepithelium was negative in all cases. NeuN was not expressed in immature neuroepithelium but was positive in all mature neural tissue within the teratoma. All adult and neonatal cortical and cerebellar tissues showed NeuN reactivity in expected neuronal subtypes (cortical neurons and cerebellar granule cells); proliferative index was low ( $<5 \%$ Ki67 and $<25 \%$ cyclin D1 staining), and OCT3/4 was uniformly negative. Conclusions: A combination of Ki67 and cyclin D1 staining is a useful adjunct to morphology in the identification of immature neural elements, which can be further confirmed by the lack of NeuN expression. In rare cases, a high frequency of cyclin D1 immunoreactivity may suggest immaturity despite low Ki67 staining, given the higher sensitivity of cyclin D1. OCT3/4 may aid in identification of other types of germ cell tumor, particularly embryonal carcinoma.

1125 BCOR Is a Robust Diagnostic Immunohistochemical Marke of YWHAE-Rearranged High-Grade Endometrial Stromal Sarcoma

Sarah Chiang, Martee Hensley, Cristina R Antonescu, Esther Oliva, Robert Soslow. Memorial Sloan Kettering Cancer Center, New York, NY; Massachusetts General Hospital, Boston, MA

Background: Recognition of high-grade (HG) endometrial stromal sarcoma (ESS) harboring YWHAE-NUTM2B gene fusions is clinically important because of its aggressive clinical behavior. While morphologic features are distinct in these tumors, they may overlap with those seen in other types of uterine sarcomas. Since BCOR mRNA upregulation has been observed in non-gynecologic sarcomas harboring YWHAE rearrangement, BCOR immunoexpression was studied in HGESS with YWHAE$N U T M 2 B$ fusion and its potential morphologic mimics to assess its diagnostic utility. Design: Immunohistochemical staining for BCOR was performed on archival wholetissue sections of 9 HGESS ( 7 and 2 with YWHAE-NUTM2B and ZC3H7B-BCOR fusions, respectively) and tissue microarrays comprised of 53 low-grade ESS, 21 endometrial stromal nodules, 26 uterine leiomyosarcomas, and 16 uterine leiomyomas. Intensity of nuclear staining (strong, moderate, weak, or negative) and percentage of positive tumor cells were recorded.

Results: All 7 HGESS with YWHAE-NUTM2B fusion showed strong nuclear staining in $>75 \%$ of tumor cells. Moderate staining in $30 \%$ of tumor cells was seen in 1 leiomyosarcoma, while weak staining in $<5 \%$ of tumor cells was seen in 6 leiomyosarcomas and 3 low-grade ESS with $J A Z F 1-S U Z 12$ fusion. No staining was observed in the 2 HGESS with $Z C 3 H 7 B-B C O R$ fusion, 21 endometrial stromal nodules, 16 leiomyomas, and all remaining 20 leiomyosarcomas and 50 low-grade ESS.

Conclusions: BCOR immunohistochemical staining is a highly sensitive marker for HGESS with YWHAE rearrangement.

1126 Genomic Copy Number Profiling of Uterine Malignant Mixed Müllerian Tumor Identifies a Recurrent Focal Amplicon Associated with Sarcomatous Transformation

Michael Herman Chui, Cherry Have, Lien N Hoang, Patricia Shaw, Cheng-Han Lee, Blaise Clarke. Toronto General Hospital, University of Toronto, Toronto, ON, Canada; BC Cancer Agency, Vancouver, BC, Canada.

Background: Uterine Malignant Mixed Müllerian Tumor (MMMT) is a high-grade biphasic neoplasm, composed of sarcomatous elements thought to originate via transdifferentiation from high-grade endometrial carcinoma. The aim of the present study was to identify molecular genetic factors contributing to the histogenesis of MMMT. Design: To elucidate a genetic basis for sarcomatous transformation in MMMT, whole genome copy number analysis was performed on 12 cases of MMMT ( 10 serous and 2 endometrioid in the carcinoma component). For each tumor, histologic review identified well-defined areas of epithelial and mesenchymal differentiation, from which matched tissue cores were taken from the corresponding FFPE tissue blocks for DNA extraction and analysis by SNP array. The GISTIC algorithm was used to identify recurrent focal amplicons. A novel FISH probe was designed to validate a recurrent chromosomal alteration and used for analysis of a tissue microarray of an additional cohort of 97 MMMTs and other uterine sarcomas. Immunohistochemical staining for desmin was also performed on the tissue microarray.

Results: Widespread copy number alterations were identified in tumors with serous histology in the carcinoma component, while the endometrioid tumors were neardiploid. Focal amplification of $13 \mathrm{q} 32$ was identified in $6 / 12$ profiled tumours, of which 4 harboured the aberration exclusively in the sarcoma component. This result was verified by FISH against GPC5, the only gene locus within the minimal region of amplification. In all 6 cases, myogenic differentiation (i.e. rhabdomyosarcoma or leiomysarcoma) was identified in the sarcoma component. In the validation cohort, high level amplification of GPC5 (GPC5/CEP13 ratio $>2.2)$ was identified in 11/97 $(11.3 \%)$ cases (9/64 MMMTs, 1/3 rhabdomyosarcomas, 1/21 leiomyosarcomas, 0/8 adenosarcomas, $0 / 1$ undifferentiated sarcoma) and an additional $4(4.1 \%)$ cases had low level gains $(G P C 5 / C E P 13$ ratio $\geq 1.5$ but $<2.2)$. Desmin staining was detected in $11 / 15(73 \%)$ tumors with GPC5 gain/amplification.

Conclusions: We identified a novel amplification of GPC5 in uterine MMMT associated with myogenic differentiation, revealing a previously unrecognized role of this gene in mediating epithelial-mesenchymal transformation in human cancer. More broadly, this work suggests that a single genetic alteration can potentially drive stable changes in cell lineage commitment. Functional studies in cell culture models are currently underway.

1127 Immune Checkpoint Status and Tumor Microenvironment in Vulvar Squamous Cell Carcinoma

Margaret Cocks, Erik G Jenson, James A Miller, Maria Del Carmen Rodriguez Pena, Aline C Tregnago, Diana Taheri, Rajni Sharma, Russell Vang, George J Netto. Johns Hopkins University, Baltimore, MD.

Background: Vulvar squamous cell carcinoma (vSCC) accounts for 5\% of female genital tract cancers. Standard therapy involves radical vulvectomy and is often associated with significant morbidity. Drugs targeting immune checkpoints such as programmed death receptor-1 (PD-1) or its ligand programmed death receptor-ligand 1 (PD-L1) have been used successfully in various solid tumors. We examined the PDL1 expression in tumor cells and tumor microenvironment immune cells (IC). We also assessed the proportion of PD-1, CD8 and FOXP3 expression in IC.

Design: Twenty-one cases of invasive vSCC were retrieved from surgical pathology archives (2005-2016). Paired inguinal lymph node metastases were available in 4 cases. Whole slides of FFPE sections were used to perform IHC analysis for PD-L1 (SP142, Ventana), PD-1 (Cellmark), CD8 (Cellmark), and FOXP3 (eBioscience-14-4777). PDL1 membranous staining was evaluated in tumor cells and IC. Extent of PD-1, CD8 and FOXP3 was classified as mild $(<10 \%)$, moderate $(10-49 \%)$ or brisk $(>50 \%)$ in IC Findings were correlated with clinicopathologic parameters.

Results: Overall, 9/21 (43\%) of vSCCs had positive PD-L1 expression in tumor cells. 18/21 (86\%) cases showed positive PD-L1 staining in IC. PD-L1 staining was observed in both primary tumor and matched metastasis in 3/4 (75\%) cases. PD-L1 correlated with patient race $(\mathrm{p}=0.043)$ but was not significantly associated with any other clinicopathologic parameters.

PD-1 was observed in intratumoral and/or stromal IC in 20/21 (95\%) cases. Moderate to brisk PD-1+ was present in 8/9 (89\%) PD-L1(+) tumors and 6/12 (50\%) PD-L1(-) tumors.

FOXP3 and CD8 staining was observed in IC in all 21 tumors as summarized in table 1. Moderate to brisk FOXP3 activity was observed in 6/9 (67\%) PD-L1(+) cases compared to 9/12 (75\%) PD-L1(-) tumors. Expression of PD-1, CD8 and FOXP3 did not correlate with clinicopathologic parameters.

\begin{tabular}{|c|c|c|c|}
\hline & \multicolumn{3}{|c|}{ Moderate - Brisk IC Expression (\%) } \\
\hline PD-L1 Tumor Cells Expression (n) & PD-1 & CD8 & FOXP3 \\
\hline Positive (9) & $8 / 9(89)$ & $9 / 9(100)$ & $6 / 9(67)$ \\
\hline Negative (12) & $6 / 12(50)$ & $11 / 12(92)$ & $9 / 12(75)$ \\
\hline
\end{tabular}

Conclusions: Nearly half of the vSCCs examined were positive for PD-L1 expression in tumor cells with over $80 \%$ demonstrating PD-L1 positivity in tumor associated immune cells. Significant expression of PD-L1 supports further investigation into the use of immunotherapy targeting the PD-1/PD-L1 pathway in vSCC.

1128 Genomic Index Assessment in Uterine Smooth Muscle Lesions: A Prognostic Tool Predictive of Recurrence. A Comprehensive Array-CGH Analysis of $\mathbf{7 7}$ Tumours

Sabrina Croce, Agnes Ribeiro, Tom Lesluyes, Jean-Christophe Noel, Mojgan Devouassoux-Shisheboran, Laurent Arnould, Gaëtan MacGrogan, Frédéric Chibon. Institut Bergonié, Bordeaux, France; Erasme University Hospital, Bruxelles, Belgium; Centre Hospitalier Lyon Sud, Pierre Bénite, Lyon, France; Centre JF Leclerc, Comprehensive Cancer Centre, Dijon, France.

Background: The diagnosis of uterine smooth muscle tumours could be in some cases particularly challenging. Few years ago we proposed a genomic approach complementary to the morphology based on the evaluation of the genomic complexity (Genomic Index, GI) (Croce et al 2015). The goal of the present study is to correlate the GI of a large series of uterine smooth muscle tumours (from benign to malignant) with clinical follow up.

Design: The genomic profiles of 77 uterine smooth muscle tumours: 10 leiomyomas (LM), 23 smooth muscle tumours with uncertain malignant potential (STUMP) and 44 leiomyosarcomas (LMS) were analysed by array-CGH.

Results: At the CGH analysis the uterine smooth muscle tumours showed a spectrum of genomic profiles varying from simple (GI mean value 1.82 ) in the LM to more rearranged in the STUMP (GI mean value 22.4) to very complex in the LMS group (GI, mean 55). At the Kaplan Meyer analysis these tumours show a risk of recurrence that is proportional to the genomic complexity, correlating to GI. At the multivariate analysis the genomic alterations associated with a lower Overall Survival (OS) and lower Progression Free Survival (PFS) were the gain of $5 p$ and $18 p$ regions and interestingly the $5 p$ region gain was the second prognostic factor for LMS after stage for OS and PFS.

Figure 1. $5 \mathrm{p}$ gain and overall survival and metastases free survival in uterine LMS

$5 p$ GAIN

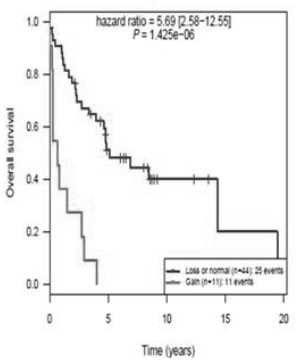

5 p GIN

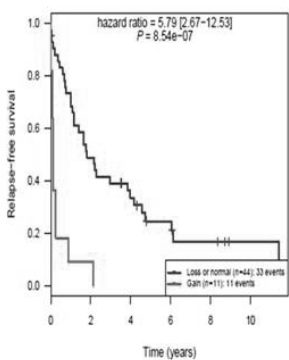


Conclusions: Uterine LMS have a complex genomic profile with a high number of chromosomal rearrangements. Based on this evidence since 5 years we have analysed and compared to the clinical evolution uterine smooth muscle tumours (Croce et al 2015) and with a comprehensive series of cases we have observed that, with the exception of bizarre nuclei LM, there is a correlation between the GI and the risk of recurrence. Here we propose a new perspective of classification of uterine smooth muscle tumours according to the morphology integrated with GI correlating with the risk of recurrence.

1129 The Immune Microenvironment of Microsatellite Stable Endometrial Carcinomas: Identification of Subsets with Higher PD-L1 Expression

Suzanne Crumley, Michael T Tetzlaff, Katherine Kurnit, Russell R Broaddus. M.D. Anderson Cancer Center, Houston, TX.

Background: Endometrial carcinomas (EC) with high levels of microsatellite instability (MSI-H) have a more intense inflammatory infiltrate than microsatellite-stable (MSS) tumors. However, inflammatory cells are still present in MSS tumors, and thus a subset of MSS tumors may have immune microenvironment changes more like MSI-H cancers. Our goal was to better characterize PD-L1 expression in MSS EC

Design: We identified $381 \mathrm{EC}$ with MSI and/or mismatch repair IHC performed. Grade and histotype were held constant for more optimal evaluation of PD-L1's association with clinical and pathological variables. 132 MSS, FIGO grade 2 EC with pure endometrioid histology were used for this study. Positive PD-L1 IHC was defined as membranous staining in tumor cells and was graded on a scale of $1+$ (faint), $2+$ (moderate) and $3+$ (strong). PD-L1 expression was analyzed in association with a variety of clinical-pathological variables.

Results: PD-L1 was positive in 48\% (63/132) of the tumors. Staining was focal (1\%) and intensity was weak to moderate $(1-2+)$ in the majority of cases. Positive PD-L1 was significantly associated with lymphatic/vascular (LVSI) and/or myometrial invasion, advanced stage, and loss of PTEN IHC expression. Loss of PTEN is common in EC, and it is linked to local immune suppression in melanoma. PD-L1 staining was especially prominent in foci of squamous metaplasia, free cells in tumor lumens, LVSI, and within the invasive tumor component. 21 cases ( $16 \%$ of total) with $\geq 2 \%$ of tumor staining for PD-L1 and/or strong (3+) tumor positivity were identified; these showed especially strong associations with similar variables, especially in LVSI/tumor invasion.

Table 1. Clinicopathologic characteristics associated with PD-L1 expression in MSS endometrial endometrioid adenocarcinomas

\begin{tabular}{|c|c|c|c|c|}
\hline & $\begin{array}{c}\text { Tumor PD-L1 } \\
\text { negative } \\
(69 / 132 ; 48 \%)\end{array}$ & $\begin{array}{c}\text { Tumor PD-L1 } \\
\text { positive } \\
(63 / 132 ; 52 \%)\end{array}$ & $p$-value & $\begin{array}{l}\text { Subgroup with } \\
\text { Increased Tumor } \\
\text { PD-L1 Positivity } \\
\text { (21/63 PD-L1 } \\
\text { positive cases) }\end{array}$ \\
\hline $\begin{array}{l}\frac{\text { Patient age }}{\text { Mean }} \\
\text { Median }\end{array}$ & $\begin{array}{c}58.6 \text { years } \\
60 \text { years }\end{array}$ & $\begin{array}{l}59.0 \text { years } \\
60 \text { years }\end{array}$ & $\begin{array}{l}0.8259 \\
0.8571\end{array}$ & $\begin{array}{c}55.8 \text { years } \\
59 \text { years }\end{array}$ \\
\hline $\begin{array}{l}\frac{\text { Tumor size }}{\geq 2 \mathrm{~cm}} \\
<2 \mathrm{~cm} \\
\text { Unknown }\end{array}$ & $\begin{array}{c}60(90 \%) \\
7(10 \%) \\
2^{*}\end{array}$ & $\begin{array}{c}54(89 \%) \\
7(11 \%) \\
2^{*}\end{array}$ & 0.852 & $\begin{array}{c}17(81 \%) \\
4(19 \%) \\
0\end{array}$ \\
\hline $\begin{array}{l}\text { Tumor stage } \\
1 / 2 \\
3 / 4\end{array}$ & $\begin{array}{c}67(97 \%) \\
2(3 \%)\end{array}$ & $\begin{array}{l}52(83 \%) \\
11(17 \%)\end{array}$ & 0.007 & $\begin{array}{c}18(86 \%) \\
3(14 \%) \\
\end{array}$ \\
\hline $\begin{array}{l}\text { Any mvometrial invasion } \\
\text { present } \\
\text { Yes } \\
\text { No }\end{array}$ & $\begin{array}{l}44(64 \%) \\
25(36 \%)\end{array}$ & $\begin{array}{l}51(81 \%) \\
12(19 \%)\end{array}$ & 0.028 & $\begin{array}{l}18(86 \%) \\
3(14 \%)\end{array}$ \\
\hline $\begin{array}{l}\frac{\text { Lvmphovascular space }}{\text { invasion }} \\
\text { Yes } \\
\text { No }\end{array}$ & $\begin{array}{c}8(12 \%) \\
61(88 \%)\end{array}$ & $\begin{array}{l}22(35 \%) \\
41(65 \%)\end{array}$ & 0.001 & $\begin{array}{c}8(38 \%) \\
13(62 \%)\end{array}$ \\
\hline $\begin{array}{l}\text { PD-L1 positivity in stroma } \\
\text { Yes } \\
\text { No }\end{array}$ & $\begin{array}{l}42(61 \%) \\
27(39 \%)\end{array}$ & $\begin{array}{l}47(75 \%) \\
16(25 \%)\end{array}$ & 0.963 & $\begin{array}{c}17(81 \%) \\
4(19 \%)\end{array}$ \\
\hline $\begin{array}{l}\text { Staining of detached cells } \\
\frac{\text { within lumen }}{\text { Yes }} \\
\text { No }\end{array}$ & $\begin{array}{l}29(42 \%) \\
40(58 \%)\end{array}$ & $\begin{array}{c}19(30 \%) \\
44(7 \%)\end{array}$ & 0.157 & $\begin{array}{c}7(33 \%) \\
14(67 \%)\end{array}$ \\
\hline $\begin{array}{l}\frac{\text { PTEN IHC }}{\text { Positive }} \\
\text { Negative } \\
\text { Not performed }\end{array}$ & $\begin{array}{c}41(60 \%) \\
27(40 \%) \\
1^{*}\end{array}$ & $\begin{array}{c}23(37 \%) \\
40(63 \%) \\
0\end{array}$ & 0.007 & $\begin{array}{c}8(38 \%) \\
13(62 \%) \\
0\end{array}$ \\
\hline
\end{tabular}

"Excluded from denominator of total percentage calculation.

Table 2. Characteristics of PD-L1 expression in MSS endometrial endometrioid adenocarcinomas

\begin{tabular}{|c|c|c|}
\hline & $\begin{array}{c}\text { Tumor PD-L1 } \\
\text { positive } \\
\text { (63 cases) }\end{array}$ & $\begin{array}{l}\text { Subgroup with } \\
\text { Increased Tumor } \\
\text { PD-L1 Positivity } \\
\text { (21/63 cases) }\end{array}$ \\
\hline $\begin{array}{l}\text { Percent of staining } \\
1 \% \\
2-5 \% \\
6-20 \% \\
21 \%+ \\
\end{array}$ & $\begin{array}{c}43(68 \%) \\
16(25 \%) \\
3(5 \%) \\
1(2 \%) \\
\end{array}$ & $\begin{array}{c}1(5 \%) \\
16(76 \% \\
3(14 \%) \\
1(5 \%) \\
\end{array}$ \\
\hline $\begin{array}{l}\text { Strength of staining } \\
1+ \\
2+ \\
3+\end{array}$ & $\begin{array}{c}27(43 \%) \\
28(44 \%) \\
8(13 \%)\end{array}$ & $\begin{array}{c}2(10 \%) \\
11(52 \%) \\
8(38 \%)\end{array}$ \\
\hline $\begin{array}{l}\text { Invasive component and/or LVSI staining } \\
\text { Yes } \\
\text { No } \\
\text { No myometrial invasion identified on slide }\end{array}$ & $\begin{array}{c}29(69 \%) \\
13(31 \%) \\
21^{*}\end{array}$ & $\begin{array}{c}15(100 \%) \\
0 \\
6 *\end{array}$ \\
\hline $\begin{array}{l}\text { Area of tumor where staining is positive } \\
\text { Periphery only } \\
\text { Middle only } \\
\text { Both }\end{array}$ & $\begin{array}{l}15(24 \%) \\
25(40 \%) \\
23(37 \%)\end{array}$ & $\begin{array}{c}1(5 \%) \\
4(19 \%) \\
16(76 \%)\end{array}$ \\
\hline $\begin{array}{l}\text { Squamoid metaplasia staining } \\
\text { Yes } \\
\text { No } \\
\text { No squamoid metaplasia identified }\end{array}$ & $\begin{array}{c}41(91 \%) \\
4(9 \%) \\
18^{*}\end{array}$ & $\begin{array}{c}18(95 \%) \\
1(5 \%) \\
2^{*}\end{array}$ \\
\hline
\end{tabular}

Conclusions: We identified variables associated with positive PD-L1 expression in MSS EC and that a subset (16\%) has particularly strong PD-L1 expression. Therefore, limiting checkpoint blockade/immunotherapy to MSI-H EC may be too exclusive.

1130 Spectrum of PTEN Mutations in Mullerian Malignancies: Impact on PTEN Protein Expression

Suzanne Crumley, Russell R Broaddus. The University of Texas M.D. Anderson Cancer Center, Houston, TX.

Background: $P T E N$ is a tumor suppressor gene that negatively regulates the PI $3 \mathrm{~K}-\mathrm{AKT}$ pathway. Mutation may result in loss of PTEN protein expression, resulting in PI3KAKT pathway activation and providing a potential therapeutic target. Somatic PTEN mutations are especially common in endometrial endometrioid adenocarcinoma. It is not known how often mutation actually results in protein loss. Our goal was to define the specific mutations in PTEN identified in a clinical setting and determine how these impact protein expression.

Design: Clinical next-generation sequencing (NGS) was performed on 1,192 patient tumors using 46,50, or 409 multi-gene panels. 117 cases had an alteration in the PTEN gene and PTEN IHC performed. 76 of the PTEN mutant tumors were from a Müllerian primary site. The primary sites included uterus (62), cervix (5), ovary (6), vagina (2), and endometriosis of colon (1). 96 PTEN mutations were identified in the 76 cases, with 17 patients having more than one PTEN mutation. These 76 cases were reviewed in detail to identify any associations between the type of PTEN alteration and PTEN IHC expression pattern.

Results: Overall, 44/76 (58\%) of the PTEN mutant tumors had associated PTEN protein loss. The majority of cases with multiple PTEN mutations were in tumors of the pure endometrioid histotype $(11 / 17,65 \%)$, and $14 / 17(82 \%)$ were in patients with some component of endometrioid histology. Nonsense mutations were more often associated with protein loss, but protein loss was also associated with a relatively high percentage of missense and splice mutations. Tumor histology had no significant effect on type of mutation or incidence of associated PTEN protein loss.

Table 1. Classes of PTEN mutations in Mullerian primary tumors.

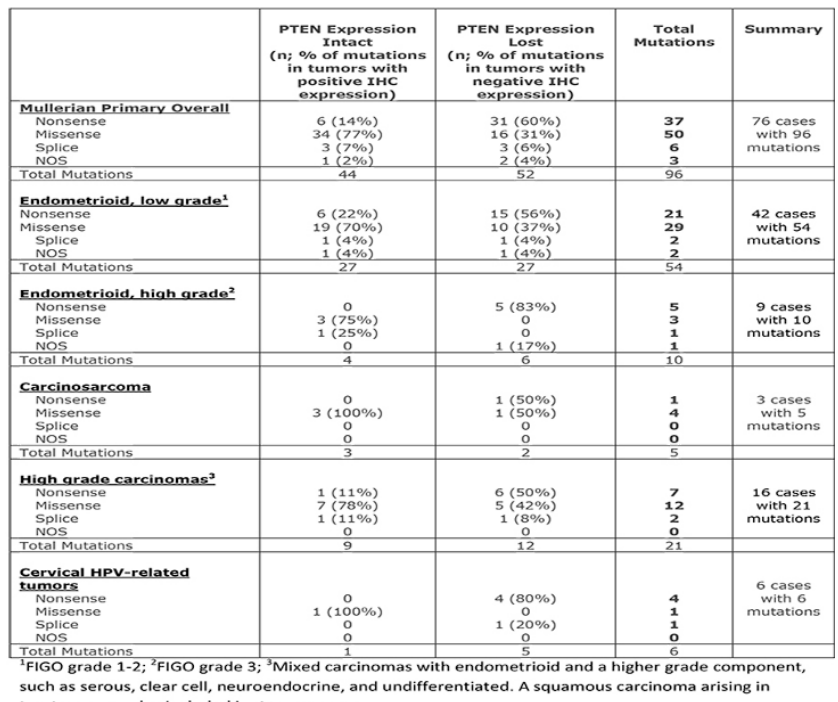

teratoma was also inclued in uns cotego,

Conclusions: PTEN somatic mutations are common in tumors of Mullerian origin, particularly in the endometrioid histotype. However, PTEN mutation does not correlate with protein loss in approximately half of the cases. Specific mutation pattern cannot reliably predict protein expression. Therefore, we recommend that immunohistochemistry be used to follow-up on PTEN mutations detected in clinical NGS panels in tumors of Mullerian origin. Bioinformatics can be used to help predict which mutant tumors with intact protein expression are potentially expressing nonfunctional protein.

1131 Clear Cell Carcinoma of the Endometrium: Evaluation of Prognostic Parameters in a Multi-Institutional Cohort of 165 Cases

MHD Fayez Daaboul, Eman Abdulfatah, Oumaima Chaib, Marcel T Ghanim, Baraa Alosh, David G Mutch, Sean C Dowdy, Sudeshna Bandyopadhyay, Rouba Ali-Fehmi. WSU, Detroit, MI; Washington University, St. Louis, MO; Mayo Clinic, Rochester, MN. Background: Clear cell carcinoma(CCC) is an aggressive subtype of endometrial carcinoma. The aim of this study was to evaluate the clinicopathological features of a large multi-institutional cohort of CCC in order to identify prognostically significant parameters.

Design: A retrospective review of endometrial CCC $(n=165)$ from 1995-2012 at 3 institutions was conducted to correlate patient's age, race, tumor size, stage, grade, myometrial invasion (MI), lymphovascular invasion (LVI), lymph node (LN) and adnexal involvement (AI), adjuvant therapy with clinical outcomes using Cox-regression statistical analysis.

Results: Patients' ages ranged from 36- 90 (median 67) years. CCC was more common in Caucasian (CA) vs. African Americans ( $70 \%$ vs. $30 \%$ ). The median tumor size was $3.6 \mathrm{~cm}$ with $80 \%$ measuring $>2 \mathrm{~cm}$. Inner half MI was present in $44 \%$, LVI in $34 \%$, AI in $16 \%$ and $\mathrm{LN}$ metastasis in $30 \%$ of cases. $58 \%$ of the patients presented with early 
stage disease. Lymphadenectomy was performed in $82 \%$ while omentectomy in $52 \%$ of patients. Radiotherapy was part of the adjuvant therapy in $48 \%$ while $34 \%$ received adjuvant chemotherapy. The 5 -year overall survival (OS) was $58 \%$. Significantly better 5-year OS was noted with age $<70$ years, early stage, absence of AI and lack of recurrence $(\mathrm{P}=0.018, \mathrm{P}=0.005, \mathrm{P}=0.005$ and $\mathrm{P}=0.005$, respectively). By Cox-regression analysis, advanced stage disease, old age and adnexal involvement were significant independent predictors of worse disease free interval (DFI) $(\mathrm{P}=0.001, \mathrm{P}=0.005$ and $\mathrm{P}=0.019$, respectively) whereas inner half $\mathrm{MI}$ was a significant independent predictor of longer DFI $(\mathrm{P}=0.004)$. Adjuvant radiotherapy alone was a significant independent predictor of better 5 -year $\mathrm{OS}(\mathrm{P}=0.012)$.

\begin{tabular}{|c|c|c|}
\hline & DFIHR $(95 \%$ CI $)$ & P value \\
\hline Age 70 vs $>70$ & $2.20(1.27-3.82)$ & 0.005 \\
\hline StageI-II vs III-IV & $7.09(3.18-15.82)$ & 0.001 \\
\hline Depth of MIInner vs outer half & $0.35(0.14-0.69)$ & 0.004 \\
\hline Adnexal involvementPresent vs absent & $2.23(1.14-4.34)$ & 0.019 \\
\hline Adjuvant radiotherapyYes vs no & $0.72(0.32-1.61)$ & 0.425 \\
\hline Adjuvant chemotherapyYes vs no & $1.10(0.48-2.50)$ & 0.815 \\
\hline Adjuvant chemo- and radiotherapyYes vs no & $0.71(0.21-2.35)$ & 0.575 \\
\hline
\end{tabular}

Conclusions: Endometrial CCC was found to be more common in older CA women. Old age at diagnosis, advanced stage, deep MI and adnexal involvement were independent poor prognostic factors. Adjuvant radiotherapy had a significant positive impact on 5 -years OS.

\section{Intraoperative Pathologic Consultation on Hysterectomy Specimens for Endometrial Cancer: An Assessment of the Accuracy of Frozen Sections, Gross Only Evaluations and Obtaining Random Sections of a Grossly Normal Endometrium}

Mohamed M Desouki, Zaibo Li, Omar Hameed, Oluwole Fadare. Vanderbilt University Medical Center, Nashville, TN; Ohio State University, Cincinnati, OH; University of California San Diego, San Diego, CA.

Background: Pathologic intraoperative consultation (IOC) is a common approach for segregating the subset of endometrial cancer patients that likely require a lymphadenectomy. In this study, we evaluate factors related to the performance and value of IOC in endometrial cancer, including the accuracy of frozen sections, "gross only" examinations and obtaining random sections when a gross lesion is not apparent Design: Dataset was aggregated from 3 institutions, and was comprised of 205 consecutive cases in which an IOC was requested on a hysterectomy specimen

Results: IOC was performed by gross examination only in 17/205 (8\%) of cases. Using the final diagnoses as gold standard, a "gross only" determination that a lesion "suspicious for malignancy" is present proved to be $67 \%$ sensitive and $100 \%$ specific. There was a $100 \%$ concordance between a gross only IOC and final interpretation regarding myometrial invasion (MI). Among the 64 cases wherein a gross lesion was not apparent and random sections were examined intraoperatively, a final diagnosis of carcinoma was rendered in 20 . In $3(15 \%)$ of those 20 cases was a diagnosable malignancy on the random section. 19 of the 20 cases were classified as grade 1 endometrioid carcinoma with $<50 \% \mathrm{MI}$, with the 20th case being a serous carcinoma that was not captured on the random section reviewed intraoperatively. The frozen section/final diagnosis concordance rate regarding histologic grade was $72 \%$ (improved to $80 \%$ in a 2 -tiered grading system). The frozen section/final diagnosis concordance rate for diagnostic categories (benign, hyperplasia, carcinoma) was $91 \%$. Determining the depth of MI was most problematic: $36 \%$ of cases with a final interpretation of $\geq 50 \%$ MI had been interpreted as $<50 \%$ on the frozen sections.

Conclusions: In the presence of a gross lesion suspicious for malignancy, a "gross only" examination is an acceptable approach for identifying MI but insufficiently sensitive to confirm that a malignancy is present. Obtaining random sections in the absence of gross lesion has no significant benefit, and a negative result is likely to provide potentially inaccurate data to the surgeon. Frozen section analyses are generally reliable in defining pathologic risk parameters, but they also display significant limitations that should be considered.

\section{Frequent Aneuploidy Detection in Non-Molar Abortuses by Molecular Analysis of Products of Conception with Atypical Villus Morphology}

Jessica Dillon, Jorge L Gonzalez, W T Parks, Laura Tafe. Dartmouth Hitchcock Medical Center, Lebanon, $\mathrm{NH}$.

Background: A clear distinction between early non-molar abortus (NMA) and partial hydatiform mole (PM) can be difficult by histology alone given the overlapping features of atypical villus morphology (AVM) including edematous chorionic villi with variation of trophoblastic hyperplasia and proliferation that can be seen in both entities. Multiple additional modalities have been proposed to aid in this distinction, including flow cytometry, fluorescence in situ hybridization (FISH) and SNP array analysis, but the optimal molecular approach has not yet been defined. In our institution, we utilize a combination of FISH and SNP array analyses. The goal of this retrospective study was to examine the utility of these molecular analysis to confirm the diagnosis of PM and, in addition, identify clinically significant aneuploidy that likely also correlates with AVM. Design: The Department of Pathology Archives was searched for products of conception (POC) from 2009-present which had undergone molecular analysis. Chromosomal aneuploidy analysis was performed on formalin-fixed paraffin-embedded (FFPE) POC tissue using fluorescence in situ hybridization (FISH) for chromosomes 13 (13q14), 15 (D15Z4), 16 (D16Z3), 18 (D18Z1), 21 (21q22), 22 (22q11), X (DXZ1), and Y (DYZ3) (send out test). SNP array analysis (OncoScan, Affymetrix) was also performed on the FFPE in a subset of cases. Final diagnoses were rendered as NMA or PM.
Results: We identified 83 POCs with molecular analysis results. Sixty cases (60/83, $73 \%)$ were diagnosed as NMA and $23(23 / 83,27 \%)$ as PM with triploidy. Thirtyfour $(34 / 60,57 \%)$ of NMA were genotyped as diploid, three cases $(3 / 60,5 \%)$ were monosomy $\mathrm{X}$, and the remaining $23(23 / 60,38 \% ; 28 \%$ of total cases) showed trisomy in chromosomes: 13 (1), 15 (3), 16 (9), 17 (1), 18 (4), 21 (3) and 22 (2). One case of PM showed triploidy XXYY, +13 . SNP array analysis was performed in 20 cases and corroborated the FISH findings in each case.

Conclusions: Selecting POCs for additional molecular analysis based on AVM resulted in a high detection rate of abnormal chromosomal alterations in 49/83 (59\%), including complex karyotypes, with aneuploidy in $38 \%$ of NMA cases tested. Recognizing AVM in evaluation of even spontaneous POCs may allow for detection of clinically relevant aneuploidy. Accurate identification holds the potential to initiate genetic testing earlier in patients, particularly those with recurrent pregnancy losses.

1134 Pathologic Ultrastaging of Endometrial Cancer Sentinel Lymph Nodes: The Dartmouth-Hitchcock Medical Center Experience

Jessica Dillon, Jorge L Gonzalez, Evelyn L Fleming, Laura Tafe. Dartmouth-Hitchcock Medical Center, Lebanon, NH.

Background: The prognosis of endometrial cancer (EC) is largely dependent on tumor stage. Given the morbidity associated with lymphedema, alternative methods to avoid total lymphadenectomy have been investigated in EC. Sentinel lymph node (SLN) mapping is used for staging melanomas and breast cancers, and in 2015 our institution instituted SLN mapping for EC. We report the outcome of our experience, correlate with histology, depth of myometrial invasion, tumor grade and the uncertain benefits of immunohistochemistry (IHC) staining in histologically negative lymph nodes.

Design: Intraoperatively, SLNs were identified via mapping after cervical injection with indocyanine green and submitted to Pathology. All SLNs were processed according to the ultrastaging protocol developed at Memorial Sloan Kettering (PMID: 23694985). SLN are serially sectioned and submitted entirely. When initial H\&E slides were negative for tumor metastasis, two additional deeper recuts and a pancytokeratin stains were performed. Isolated tumor cells were reported if the lymph nodes displayed less than or equal to 10 isolated, cytokeratin positive tumor cells. A retrospective database search was performed for identification of endometrial carcinoma tumors with associated SLN mapping.

Results: A total of 109 women, ages 33 to 102 (mean 64 years old) were identified. The majority of tumors were endometrioid histology $(98 / 109,90 \%)$ and the tumor size ranged from 0.3-9.1 cm (mean $4.0 \mathrm{~cm}$ ). Fifty-five tumors were FIGO grade I $(50 \%)$, 36 grade II $(33 \%)$, and 7 grade III $(6 \%)$. The majority of tumors were stage pT1a $(78 / 109,72 \%)$. Sentinel nodes were mapped and removed from pelvic, obturator, iliac, parametrial, and hypogastric sites. Two cases were positive for macrometastasis $(1 \%)$ and 8 were positive $(7 \%$; stage $\mathrm{Tla}-4, \mathrm{~T} 1 \mathrm{~b}-3, \mathrm{~T} 2-1)$ for isolated tumor cells using IHC cytokeratin staining. The remaining SLNs were all negative for tumor $(92 \%)$.

Conclusions: Reports suggest that SLN mapping and use of IHC to identify micro metastatic foci of EC will improve detection and staging and avoid a total lymphadenectomy. In our experience, further processing of histologically negative SLN did not improve detection of macro- or micrometastases or alter the tumor stage. Furthermore, within the gynecologic oncology community, at there is a lack of consensus on how to treat patients with isolated tumor cells, thereby questioning the utility of cytokeratin IHC in SLNs.

1135 ID4 Allelic Variant Is Associated with Endometriosis and May Affect Mesothelial Epithelial-Mesenchymal Transition

Daniel Dolderer, Colin Bergstrom, Terry Morgan. OHSU, Portland, OR.

Background: Endometriosis affects approximately $10 \%$ of reproductive age women and is a leading cause of pelvic pain and infertility. This common disease is familial and genome wide association studies have suggested a potential risk locus called inhibitor of DNA binding (ID)-4 [rs6907340]. The ID4 minor allele variant (mav) is especially interesting to our group, because this tumor suppressor gene may be involved in the BRCA pathway and the epithelial-mesenchymal transition (EMT) process.

Design: Retrospective case:control study of all endometriosis cases diagnosed by tissue biopsy at OHSU from 2003-2010. Inclusion criteria required white non-Hispanic race (for allelic variant analysis), documented stage of disease severity (ASRM, stage 1 or 2 [mild], stage 3 or 4 [severe]), history of infertility (yes or no), and at least five years of documented clinical outcomes to evaluate for disease persistence and de novo presentation of breast cancer or adnexal cancer (ovary or fallopian tube). This yielded 180 cases for analysis. Negative controls were white non-Hispanic women with archived tissue biopsies for DNA and no history of endometriosis $(\mathrm{n}=177)$. DNA was extracted from FFPE tissue blocks and ID4 allelic discrimination was performed using Taqman. All samples were tested in duplicate and only reproducible genotypes were included for analysis. As a pilot study, we also immunostained a series of pelvic peritoneal biopsies for cytokeratin 7, E-cadherin, and N-cadherin, from endometriosis cases homozygous for the ID4 mav ( $\mathrm{n}=7)$ compared with negative controls.

Results: As expected, infertility was more common in cases of severe endometriosis $(12 / 52,23 \%)$ than cases of mild endometriosis $(7 / 128,5 \%)$, Fisher exact $\mathrm{p}=0.001$. The ID4 minor allele frequency in white controls was 0.40 (expected 0.39 ). The frequency was increased in endometriosis $(0.45)$ and more so in severe disease cases (0.47), yielding an odds ratio of $1.6[0.99-2.75], \mathrm{p}=0.05$. The ID4 variant may also be a risk factor for breast/adnexal cancer (11\% of CT/TT cases compared with $7 \%$ of CC homozygotes), odds ratio 1.82 [0.38-8.82]. In addition, our pilot IHC experiment suggested pelvic peritoneal mesothelial cells from cases may be more likely to lose E-cadherin staining and gain N-cadherin signal, consistent with EMT.

Conclusions: Although the functional significance of the ID4 rs6907340 allelic variant is unknown, we observed significant differences in allele frequency related to the severity of endometriosis and potentially cancer risk. 
1136 Discrepancy Between Morphology, and p16 and Ki-67 Expression in Squamous Intraepithelial Lesions of the Lower Female Genital Tract

Tsetan Dolkar, Anais Malpica, Preetha Ramalingam. MD Anderson Cancer Center, Houston, TX.

Background: P16 and Ki-67 aid in the diagnosis (dx) of squamous intraepithelial lesions (SILs) of the lower female genital tract (LFGT). However, discrepancies between the expression of these markers and the morphology can occur. In this study, we present our experience with 23 such cases.

Design: 23 cases potentially classifiable as either low or high grade SIL (LSIL/HSIL) on morphology with discrepant p16 and Ki-67 results were reviewed. Discrepancies were classified in 4 categories (Cat): 1) atypical morphology, favor HSIL, but with no increase in $\mathrm{p} 16$ or Ki- $67 ; 2$ ) atypical morphology with diffuse $\mathrm{p} 16$, but with no increase in Ki-67;3) atypical morphology with increased Ki-67, but no or patchy p16;4) atypical morphology, favor reactive, but with diffuse $\mathrm{p} 16$ and/or no increase in Ki-67. Outcomes were grouped as "HSIL", "LSIL", "USIL" (undetermined SIL), and "No SIL".

Results: Patients' (pts') age range 23-84, mean 45. All were biopsies (bxs) from: cervix (12), vagina (7), vulva (3), and perianal region (1). Previous history (hx) of squamous carcinoma (SC) (5), HSIL (4), LSIL (11) and no hx of SIL/SC (3). Also, 2 cases had a hx of bone marrow transplant (BMT)- 1 in Cat 2 and 1 in 3.8 pts had pre-bx HRHPV status and they were all $+.11 \mathrm{pts}$ had post bx HPV status and all but 1 was negative. The mean length of follow up (f/u) was 15.8 months (mos) (range 1-60 mos). The Cat distribution and outcomes are summarized in Table 1.

\begin{tabular}{|l|l|l|l|l|l|}
\hline & \multicolumn{5}{|c|}{ Final outcome } \\
\hline Discrepancy Cat. & HSIL & LSIL & USIL & No SIL & Total \\
\hline 1 & $1(25 \%)$ & $2(50 \%)$ & 0 & $1(25 \%)$ & $4(17.5 \%)$ \\
\hline 2 & $5(38 \%)$ & $3(23 \%)$ & $1(8 \%)$ & $4(31 \%)$ & $13(56.5 \%)$ \\
\hline 3 & 0 & $2(67 \%)$ & 0 & $1(33 \%)$ & $3(13 \%)$ \\
\hline 4 & $1(33 \%)$ & $1(33 \%)$ & 0 & $1(33 \%)$ & $3(13 \%)$ \\
\hline
\end{tabular}

There was no correlation between the pts' $h x$ and HPV status on the outcome. In Cat 2, all the pts with HSIL had hx of SC or SIL; however, so did 3 of 4 cases who had no SIL. Only 1 case remained as USIL- a Cat 2/BMT case. The other BMT case was in Cat 3 and f/u showed exuberant condyloma - adjacent to other condylomas, 1 with focal HSIL. Conclusions: Cat 2 had most cases, followed by Cat 1 . Diffuse p16 in the absence of increased Ki-67 may be seen in lesions ranging from HSIL to metaplastic lesions. Close $\mathrm{f} / \mathrm{u}$ is warranted in Cat 2 cases as HPV status and clinical hx cannot be relied upon. Cat 3 lesions can be a diagnostic pitfall for HSIL and may represent condylomas with tangential sectioning. No trends were observed in Cat 1 and 4 . Additional studies are needed to confirm our observations.

1137 Quantitative Assessment of the Immune Microenvironment in Ovarian Cancer: A Comparison of Two Digital Image Analysis Platforms Kai Duan, Ramlogan Sowamber, Trevor McKee, Amit Oza, Stéphanie Lheureux, Blaise Clarke, Patricia Shaw. University Health Network, Toronto, ON, Canada.

Background: The presence of tumor-infiltrating lymphocytes (TILs) in high-grade serous carcinoma (HGSC) is an important predictor of patient survival and of response to emerging immune therapies. However, immune infiltrate type, density and location in HGSC have not been well-characterized and quantification is not standardized in clinical practice. Accurate analysis of TILs has become more accessible with digital analytical techniques which have been applied successfully to determine a prognostic score in other cancer types. We have compared multiple methods of immunoscoring in a set of HGSC cases with known clinical outcomes, including two automated image analysis platforms, the widely applied Definiens Immunoscore algorithm and the Aperio Image Analysis platform.

Design: Immunohistochemistry for $\mathrm{CD} 3, \mathrm{CD} 8, \mathrm{CD} 68$, FoxP3 was applied to representative tumor sections of $42 \mathrm{HGSC}$ cases with known treatment and clinical parameters. Stained slides were scanned with the Aperio ScanScope XT scanner. Each slide was annotated for at least 4 areas of tumor, excluding stroma and normal tissue. Image analysis using both Aperio and Definiens systems quantified intratumoral TILs and assessed staining intensity (absent, low, medium, high). Cell densities (number of positive nuclei per $\mathrm{mm}^{2}$ of tissue analyzed) were recorded for each sample.

Results: Comparison of immune cell densities (ICDs) across platforms showed significant correlation for medium/high intensity staining cells $(\mathrm{r}=0.31, \mathrm{p}=0.002)$, and non-significant correlation for low intensity staining cells $(\mathrm{r}=0.05, \mathrm{p}=0.62)$. Interplatform agreement of ICD for medium/high intensity staining cells varied depending on the localization of the expression (nuclear vs cytoplasmic): FoxP3 ( $r=0.91, \mathrm{p}<0.001$ ), CD3 ( $r=0.90, \mathrm{p}<0.001), \mathrm{CD} 8(\mathrm{r}=0.87, \mathrm{p}<0.001)$ and CD68 $(\mathrm{r}=0.28, \mathrm{p}=0.09)$. There was near-perfect correlation across platforms for the area of tumor analyzed $(\mathrm{r}=1.00$, $\mathrm{p}<0.001$ ), with a mean of $23.56 \mathrm{~mm}^{2}$ per case.

Conclusions: Our study shows that there is consistency between platforms for nuclear markers. In particular, automated quantification of cell densities is more consistent for medium/high intensity than for low intensity staining cells. Further validation studies are needed to compare with manual IHC analysis. Nevertheless, digital image analysis is a promising avenue to enhance prognostication and selection of patients for novel immune therapies in HGSC, but the success may be biomarker dependent.
1138 Profiling of Tumor Mutational Burden (TMB), Microsatellite Instability (MSI), and PD1/PD-L1 Immunohistochemistry (IHC) in Gynecological Tumors

Julia A Elvin, Michael E Goldberg, Laurie M Gay, John Liao, Alessandro D Santin, James Suh, Jo-Anne Vergilio, Shakti H Ramkissoon, David Fabrizio, Garrett Frampton, Philip Stephens, Jeffrey Ross. Foundation Medicine, Cambridge, MA; Albany Medical College, Albany, NY; Yale University School of Medicine, New Haven, CT; University of Washington School of Medicine, Seattle, WA.

Background: PD1/PD-L1 IHC, TMB, and MSI are biomarkers that can predict response to checkpoint inhibitors (ICPi). We analyzed 260 clinical gynecological tumors by comprehensive genomic profiling (CGP) to assess TMB and MSI status and IHC for PD1 and PD-L1 expression.

Design: DNA ( $\geq 50 \mathrm{ng}$ ) was extracted from carcinomas (Ca) of ovary/related tissues $(n=147)$, cervix $(n=24)$, and uterus $(n=45)$; sarcomas $(n=30)$; other rare neoplasms $(\mathrm{n}=14)$. CGP for up to 315 cancer-related genes plus select introns from 28 genes was used to calculate TMB [high (TMB-H; $\geq 20$ mut/Mb), intermediate (TMB-I; 6-19 mut $\mathrm{Mb}$ ) or low (TMB-L; $<6 \mathrm{mut} / \mathrm{Mb}$ )] and MSI [High (MSI-H), Stable (MSS)] status. PD1 and/or PD-L1 IHC was scored in tumor infiltrating lymphocytes (TILs) and/or tumor cells (t) for distribution $(0,1-24 \%,>24 \%$ of cells) and intensity $(0,1+, 2+)$ as negative (Neg), low positive (LP) or high positive (HP).

\begin{tabular}{|l|l|l|l|l|l|}
\hline & Ovary/Related Tissue Ca & $\begin{array}{l}\text { Cervical } \\
\text { Ca }\end{array}$ & $\begin{array}{l}\text { Uterine } \\
\text { Ca }\end{array}$ & Sarcomas & $\begin{array}{l}\text { Other } \\
\text { Tumors }\end{array}$ \\
\hline $\begin{array}{l}\text { PD-L1 LP/ } \\
\text { HP }\end{array}$ & $26.5 \%$ & $45.8 \%$ & $24.4 \%$ & $23.3 \%$ & $28.6 \%$ \\
\hline
\end{tabular}

Results: $3.6 \%$ of samples were TMB-H. TMB score did not significantly associate with PD1 status (HP/LP or Neg), but TMB-H was more common in TPD-L1 positive (HP/LP) tumors $(\mathrm{p}<0.01) ; 1.2 \%$ of PD-L1 Neg were TMB-H. CD274 amp was observed in 1 sample $(0.4 \%)$, a tPD-L1 HP ovarian Ca. MSI status did not significantly correlate with either PD1 or PPD-L1 IHC status, although there is a trend for MSI-H status in PD-L1 positive samples ( $\mathrm{p}=0.064)$. MSI-H strongly correlates with TMB-H $\left(\mathrm{p}=3.6 \times 10^{-11}\right)$. Patients who responded to ICPi with TMB-H/PD1 Neg and/or PD-L1 Neg IHC will be presented.

\begin{tabular}{|l|l|l|l|l|l|l|}
\hline & \multicolumn{3}{|l|}{ PD1 Lymphocyte Compartment } & \multicolumn{3}{l|}{ PD-L1 Tumor Compartment } \\
\hline & $\begin{array}{l}\text { Neg (n=66; } \\
30.3 \%)\end{array}$ & $\begin{array}{l}\text { LP }(\mathrm{n}=110 ; \\
50.5 \%)\end{array}$ & $\begin{array}{l}\text { HP }(\mathrm{n}=42 ; \\
19.3 \%)\end{array}$ & $\begin{array}{l}\text { Neg } \\
(\mathrm{n}=173 ; \\
69.5 \%)\end{array}$ & $\begin{array}{l}\text { LP }(\mathrm{n}=68 ; \\
27.3 \%)\end{array}$ & $\begin{array}{l}\text { HP } \\
(\mathrm{n}=8 ; \\
3.2 \%)\end{array}$ \\
\hline $\begin{array}{l}\text { TMB-L } \\
(\mathrm{n}=190)\end{array}$ & 50 & 87 & 24 & 136 & 36 & 6 \\
\hline TMB-I (n=64) & 15 & 21 & 12 & 35 & 25 & 2 \\
\hline TMB-H (n=9) & 1 & 2 & 6 & 2 & 7 & 0 \\
\hline MSS (n=230) & 59 & 96 & 36 & 157 & 56 & 8 \\
\hline $\begin{array}{l}\text { MSI High } \\
(\mathrm{n}=13)\end{array}$ & 4 & 3 & 5 & 6 & 7 & 0 \\
\hline $\begin{array}{l}\text { MSI } \\
\text { Ambiguous } \\
(\mathrm{n}=5)\end{array}$ & 0 & 5 & 0 & 4 & 0 & 0 \\
\hline
\end{tabular}

Conclusions: Advanced gynecological tumors were stratified by TMB, MSI, PD1 and/or tPDL1 expression to identify patients who might benefit from ICPi. Additional response data to ICPi will be needed to validate the predictive power of each biomarker.

\section{A 20\% Solid Architecture Threshold Is Clinically Relevant in} Endometrial, Endometrioid Adenocarcinoma

Elizabeth Euscher, Dawen Sui, Anais Malpica. MD Anderson, Houston, TX.

Background: Grading endometrial, endometrioid adenocarcinoma (EA) is 3 tiered based on \% solid architecture (\%SA); however, 2 tier grading systems are more reproducible. Additionally, in EA with $<50 \% \mathrm{SA}$, FIGO grade 1 or 2 may not be clinically relevant. This study asks if a previously proposed $20 \%$ SA thresholdis of clinical relevance to base a 2 tier grading system for EA.

Design: 264 consecutive FIGO grade 1-3 EA (2003-2008) were evaluated for \%SA and other predictors of stage or outcome: pt age, tumor size, depth of myometrial invasion (DOI), lower uterine segment (LUS) involvement, cervical stromal (CX) invasion, pattern of myometrial invasion (microcystic/fragmented/elongated gland, MELF; single cell/small cluster, SCI), lymphovascular invasion (LVI), lymph node metastases (LNM), and extrauterine disease (EUD). Treatment and pt outcome were recorded. Based on a prior study, $20 \%$ SA was tested as a possible breakpoint for a 2 tier system. Kaplan-Meier estimates determined overall survival (OS) and time to recurrence. Cox proportional hazards regression model assessed pathologic factors. Logistic regression evaluated associations between advanced stage and pathologic predictors.

Results: Solid component ranged from 0-90\%: 0 to $\leq 5 \%, 29$ (11\%); 6-50\%, 210 (79.5\%); $>50 \%, 25(9.5 \%) .78(30 \%)$ tumors had $>20 \%$ SA. Predictors associated with LNM or EUD and outcome in the study cohort included: $>60 \mathrm{yo}, 137(52 \%)$; TS $>2 \mathrm{~cm}$, $176(76 \%)$; DOI $>50 \%$, $59(22 \%)$; LVI, $88(33 \%)$, LUS, $104(40 \%)$, CX, $92(35 \%)$, MELF, 92 (35\%), SCI, 39 (15\%), LNM 26 (10\%), EUD, $8(3 \%) .13$ pts with $<20 \%$ SA had LNM; all 13 had at least one predictor of LNM: LVI, 12; MELF or SCI, 11; DOI $>50 \%, 6$; CX, 5.11 pts with $<20 \%$ SA recurred; 1 of these pts had no adverse predictors. Univariate analyses indicated increased risk of LNM or EUD when $>20 \%$ SA present;TS $>2 \mathrm{~cm}$, DOI $>50 \%$, LVI, MELF, SCI, LUS, or CX. $>20 \%$ SA was not a predictor of LNM or EUD in the multivariate model. 93 pts died; pts $>60 \mathrm{yo}$ and presence of $>20 \% \mathrm{SA}$, $>50 \%$ DOI, LVI, LUS and EUD were more likely to die. $37 \mathrm{pts}$ recurred. In this group, $>20 \%$ SA, DOI $>50 \%$, LVI, LUS, CX and EUD were more likely to recur. 
Conclusions: Architectural grading using a threshold $>20 \%$ SA was not a predictor of LNM or EUD. When controlling for treatment, $>20 \%$ SA was associated with cancer recurrence but not OS in the multivariate model. A threshold of $20 \% \mathrm{SA}$ may be a clinically relevant predictor of pt outcome suggesting a role for a 2 tier grading system. More studies are required to determine reproducibility and clinical validity of the $20 \%$ threshold.

1140 Appendectomy in the Setting of Mucinous Neoplasia of the Ovary: Entire Submission of a Grossly Normal Appendix Is Unneccessary Elizabeth Euscher, Preetha Ramalingam, Anais Malpica. MD Anderson, Houston, TX. Background: Traditionally, an intraoperative diagnosis of mucinous neoplasm of the ovary leads to a concomittant appendectomy to exclude a primary mucinous appendiceal neoplasm. In the majority of such cases, the appendix is grossly unremarkable with no significant clinical findings. In this study, we review consecutive cases of mucinous tumors involving the ovary with concomittant appendectomy to determine the clinical value of submitting the appendix entirely.

Design: Our institutional pathology database was searched (2005-2016) for mucinous neoplasms involving the ovary with concomittant appendectomy. In all cases, the surgery was performed by a gynecologic oncologist at our institution for a presumed primary ovarian neoplasm. Cases of endocervical type mucinous neoplasia were excluded as were those in which the appendix was not entirely submitted. Size of the ovary, frozen section diagnosis for the ovary (and appendix if applicable), gross appearance of the appendix, and final diagnoses for the ovary and appendix were recorded.

Results: 129 consecutive cases were identified with a mean tumor size of $18 \mathrm{~cm}$. Intraoperative consultation on the ovarian masses was obtained in 128 cases and had the following diagnoses: mucinous neoplasm defer to permanent, 66 (51\%);mucinous neoplasm favor borderline, $30(23 \%)$; mucinous neoplasm, benign, 11 ( $8.5 \%)$; mucinous carcinoma or suspicious for carcinoma, $9(7 \%)$; mucinous neoplasm, possible metastasis, $12(9 \%)$. For cases of metastatic mucinous carcinoma to the ovary, 1 was a pancreatic primary and 12 were of appendiceal origin. Frozen section was performed on the appendix in 6 cases, 3 of which were grossly abnormal and had a primary mucinous appendiceal neoplasm. The appendix was grossly abnormal in all cases with a primary mucinous appendiceal neoplasm. 5 cases with a grossly normal appendix had one or more incidental findings: eosinophilic appendicitis (1), incidental well-differentiated neuroendocrine carcinoma (2), benign mucinous cystic neoplasm (1), serrated adenoma (1), endosalpingiosis (1)

Conclusions: All cases with an appendix containing a clinically significant primary mucinous appendiceal neoplasm were grossly abnormal. No clinically significant findings were found in cases with a grossly normal appendix. Sampling of the appendix margin, mid section and the entire distal tip is adequate. It is not necessary to entirely embed a grossly normal appendix.

1141 The Diagnosis of Mucinous Lesions in Endometrial Samplings by Gynecologic Pathologists: An Interobserver Variability Study

Oluwole Fadare, Andres A Roma, Paulette Mhawech-Fauceglia, Vinita Parkash, Joseph T Rabban. UCSD, San Diego, CA; USC, Los Angeles, CA; Yale, New Haven, CT; UCSF, San Francisco, CA.

Background: Proliferative mucinous lesions of the endometrium may be diagnostically problematic when encountered in sampling specimens, since morphologic criteria are suboptimally predictive of malignancy. The 2014 WHO classification recognizes metaplasia, carcinoma and atypical mucinous glandular proliferation (AMGP) as diagnostic categories for endometrial mucinous lesions. The current study assessed the reproducibility among gynecologic pathologists in their diagnosis of mucinous alterations in endometrial sampling specimens

Design: 26 glass slides, each of an endometrial biopsy, curettage or polypectomy specimen, were independently reviewed by 4 gynecologic pathologists from 4 academic medical centers. 20 of the cases were consecutive cases from a single institution for which the word "mucinous" was used in the diagnostic report, and 6 were a variety of uterine tissues with mucinous epithelium. Observers were given the patients' ages as well as relevant excerpts from the 2014 WHO classification, and were asked to classify each case into one of 4 groups: 1) mucinous metaplasia 2) AMGP 3) carcinoma and 4) "other" -indicative of the absence of a true mucinous alteration and/or an alteration of non-endometrial origin

Results: The overall reproducibility was "fair", with a Fleiss kappa [K] of $0.39 \pm 0.034$ In an analytic scenario that established 3 clinically significant groups (benign/ non-neoplastic, atypical, and malignant) by redistributing all group 4 answers, the resultant $\mathrm{K}$ improved to $0.51 \pm 0.04$ (moderate reproducibility). A subset analysis that only included 2 options - benign/non-neoplastic and atypical/carcinoma showed moderate reproducibility ( $\mathrm{K} 0.46 \pm 0.1$ ). Among the 7 cases that were classified as either benign cervical tissue or endometrial mucinous metaplasia by $\geq 75 \%$ of observers, the reproducibility for this binary distinction was moderate to substantial $(\mathrm{K}=0.59 \pm 0.08)$. Similarly, for the 14 cases that were classified as either carcinoma or atypical by $\geq 75 \%$ of observers, there was moderate reproducibility $(\mathrm{K}=0.53 \pm 0.07)$ in making that distinction. Only one case was classifed as benign or non-neoplastic by at least one observer and as malignant by at least one other observer

Conclusions: Gynecologic pathologists show suboptimal reproducibility in their diagnostic categorizations of mucinous alterations in endometrial sampling specimens. Reproducibility was no more than moderate, even for distinguishing categories with potentially disparate management implications
1142 Endometrial Serous Carcinoma with Pseudo-Endometrioid Features in Biopsies and Curettages: The Diagnostic Value of "Focal Apical Anaplastic Nuclei"

Oluwole Fadare, Mohamed M Desouki, Andres A Roma, Vinita Parkash, Wenxin Zheng. UCSD, San Diego, CA; Yale, New Haven, CT; Vanderbilt, Nashville, TN; UTSW, Dallas, TX

Background: A small subset of endometrial serous carcinomas (ESC) are devoid of the classical cytoarchitecture of the histotype, and instead display morphologic features that are reminiscent of low grade endometrioid carcinoma [EC]. Their appearances in endometrial biopsies, where they are most likely to be misdiagnosed, have not been previously characterized. We describe a cohort of cases herein, including an assessment of a novel feature, "focal apical anaplastic nuclei" [FAAN], as a potentially useful diagnostic feature for this distinctive variant

Design: 11 cases of ESC with $\geq 95 \%$ pseudo-endometrioid pattern were retrospectively identified from the files of multiple institutions and were studied in detail. The incidence of FAAN (anaplastic nuclei that protrude intraglandularly above the cellular apices, typically in $<10 \%$ of nuclei) was assessed in the study cases as well as a control group of 132 grades $1 \& 2 \mathrm{EC}$

Results: Patients ranged in age from 58-72 years (mean 65). 64\% of cases had been previously misdiagnosed as EC or atypical hyperplasia. Lesional glands were typically fragmented, and constituent cells were predominantly columnar with nuclear stratification, loss of nuclear polarity, and hyperchromasia. Nucleolomegaly was in $<25 \%$ of nuclei in $10 / 11$ cases. Solid or cribriform areas were absent, as was squamous, mucinous or secretory differentiation. The average MI was $8 \mathrm{MF} / 10$ HPF (but 23 $\mathrm{MF} / 10 \mathrm{HPF}$ in evaluable resections). A concurrent endometrial polyp was present in $8 / 11$ cases. Conventionally papillary areas were present focally in the biopsy in only 1 case, and in the hysterectomy in 4 cases. All were ultimately FIGO staged IA; the hysterectomy specimens showed no residual disease $(\mathrm{n}=1)$, endometrium-confined disease $(n=4)$, and $<50 \%$ myoinvasive disease $(n=6)$. All tested cases showed diffuse/ aberrant expression of p53 (11/11;2 null), p16 (7/7), and ER (7/7). FAAN was present in 9/11 ESC cases and was not seen in any of the 132 control cases of EC

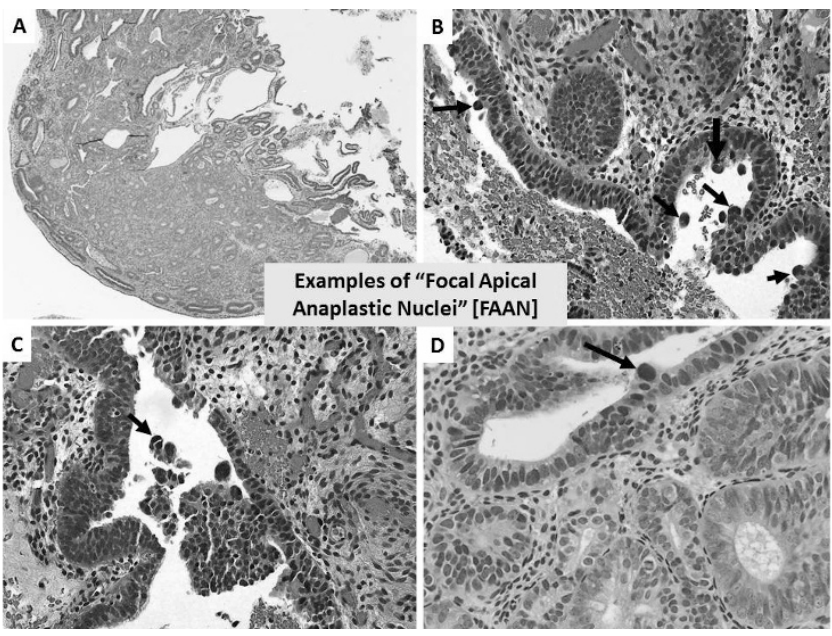

Conclusions: FAAN appears to represent an early form of the intraglandular budding of conventional ESC, and accordingly may have diagnostic value in ESC with pseudoendometrioid features

1143 The Expression of PD-L1, PD-1, and DNA Mismatch Repair Proteins in Clear Cell Carcinoma of the Ovary: A Pilot Assessment of Frequency, Clinicopathologic Significance and Potential Correlations

Oluwole Fadare, Charles M Quick, Katja Gwin, Vinita Parkash. UCSD, San Diego, CA; UAMS, Little Rock, AR; UTSW, Dallas, TX; Yale University, New Haven, CT. Background: Immune check point inhibitors have proven to be therapeutically effective for several human cancers, and the effect is at least partially modulated by microsatellite stability status in some cancers, most notably in a subset of colorectal cancers. Herein, we assess the frequency, clinicopathologic significance and interrelationships between the expression of programmed cell death protein 1 (PD-1 or CD279), its ligand PD-L1, and DNA mismatch repair proteins (MMR) in a cohort of ovarian clear cell carcinomas. Design: Immunohistochemical analyses for PD-L1, PD-1, MSH2, MSH6, PMS2, MLH1 were performed on tissue microarray slides of 54 ovarian $\mathrm{CCC}$, configured in duplicate $1 \mathrm{~mm}$ cores. PD-L1 was assessed with the SP263 clone (Roche). Both PD-1 and PDL1 were scored positive if tumor cells and/or tumor-associated immune cells showed unequivocal membranous and/or cytoplasmic staining in $\geq 5 \%$ cells. Intratumoral and peri-tumoral tumor infiltrating lymphocytes were assessed on whole tissue sections. Results: PD-1 was negative in all cases. PD-L1 was positive in $11 \%$ (6/54) of cases, and immunoreactivity was invariably confined to the tumor cells in these cases, not the associated immune cells. Abnormal MMR expression was present in $5(9 \%)$ of 54 tumors [MSH6 (n=3), MLH1/PMS2 (n=2), MLH1 (n=1)]. Patients with abnormal MMR were slightly younger (mean 46 years, range $30-67$ ) than the entire group (mean 55.1, range 30-84). There was no correlation between PD-1 or PD-L1 phenotypes and DNA MMR protein status. PD-L1 was not associated with patient age, and neither PD-L1 nor DNA mismatch repair protein status were associated with tumoral architectural patterns, mitotic index, FIGO stage [I/II vs III/IV], intratumoral/peri-tumoral infiltrating lymphocytes, or endometriosis. 
Conclusions: PD-L1 expression is present only in a small subset - $11 \%$ - of ovarian clear cell carcinomas, and is not associated with clinicopathologic factors or DNA mismatch repair protein status in this histotype. PD-1 expression was entirely absent in our cohort of cases. The therapeutic utility, if any, of immune checkpoint inhibitors for this distinctive histotype requires additional study.

1144 Simplified Immunohistochemical and Targeted Sequencing Approach Reproduces Classification of Endometrial Carcinoma into TCGA Molecular Subgroups

Daniel Fix, Sarah Chiang, Rajmohan Murali, Kay Park, Robert Soslow, Karen Cadoo, Tara E Soumerai, Vicky Makker, Sumit Middha, Carol Aghajanian, Nadeem R AbuRustum, Deborah Delair. Memorial Sloan Kettering Cancer Center, New York, NY. Background: Endometrial cancer (EC) has traditionally been classified as types I and II (Bokhman). Recent molecular analyses have shown that this is an oversimplification. The endometrial tumor cancer genome atlas (TCGA), a multi-platform analysis, identified four distinct molecular subgroups (MSG), and significant associations with progressionfree survival. These MSG consisted of 1) Ultramutated (UM)/POLE 2) Hypermutated (HM)/MSI-high 3) Endometrioid/copy-number low and 4) Serous-like/copy-number high. We sought to reproduce this classification in a series of EC using a more limited approach, to best inform prognosis and guide therapy.

Design: The cohort consists of approximately 350 cases of EC, with ongoing accrual All cases are subjected to immunohistochemistry (IHC), including the DNA mismatch repair (MMR) proteins and p53. Cases with loss of MLH1 and/or PMS2 undergo MLH1 promoter methylation testing. Next generation sequencing (NGS) targeting the exons and select introns of 468 genes was performed. Parameters derived from NGS data include: POLE mutation status, copy number variations (CNVs), microsatellite instability (MSI), as well as additional gene panel abnormalities. Based on IHC and NGS results, each tumor was placed into one of the four MSG.

Results: Presently, 74 cases were available for completed analysis. Results are summarized in the table below. Cases classified in group 1 demonstrated mutations in the exonuclease domain of POLE as well as an UM profile (100\%). Cases in group 2 were MSI-H, HM, and MMR-D by IHC ( $58.8 \%, 82.3 \%$, and $94 \%$, respectively). Group 3 cases demonstrated no significant CNVs, were MSI stable, and p53 wild type. Group 4 cases were associated with CNVs, and p53 mutations (95\%).

\begin{tabular}{|c|c|c|c|c|c|}
\hline Molecular Subgroup & 1 & 2 & 3 & 4 & All Groups \\
\hline$\%$ (total) & $12.2 \%$ & $23 \%$ & $36.4 \%$ & $28.4 \%$ & $(\mathrm{n}=74$ total) \\
\hline AverageAge (Yrs) & 57.25 & 59.7 & 61.4 & 63.2 & 61 \\
\hline FIGO Grade 1 & $15 \%$ & $10 \%$ & $30 \%$ & $0 \%$ & 20 \\
\hline FIGO Grade 2 & $8.3 \%$ & $50 \%$ & $41.7 \%$ & $0 \%$ & 12 \\
\hline $\begin{array}{c}\text { FIGO Grade 3+ (highgrade } \\
\text { subtypes) }\end{array}$ & $12.5 \%$ & $22.5 \%$ & $52.5 \%$ & $12.5 \%$ & 40 \\
\hline MSI Unstable & $9.1 \%$ & $90.9 \%$ & 0 & 0 & 11 \\
\hline Ultramutated ? Y & $100 \%$ & 0 & 0 & 0 & 9 \\
\hline TP53 Mutant? Y & $14.3 \%$ & $14.3 \%$ & $0 \%$ & $71.4 \%$ & 28 \\
\hline
\end{tabular}

Conclusions: By using IHC and a limited NGS analysis we were able to recapitulate the MSG of the TCGA. As further data accumulates, correlation between molecular classification and outcome will be performed. Classification of EC in this manner has become necessary for both prognostic and therapeutic purposes.

1145 Placental Mesenchymal Dysplasia: A Clinicopathological Study of 12 Cases with Gestational Ages Less Than 20 Weeks

Masaharu Fukunaga. Shin-Yurigaoka General Hospital, Kawasaki, Japan.

Background: Placental mesenchymal dysplasia (PMD) is characterized by placentomegaly and can be mistaken for partial hydatidiform mole (PM) or complete hydatidiform mole $(\mathrm{CM})$ with twin both clinically and macroscopically because of the presence of "grapelike vesicles". It may be associated with Beckwith-Wiedemann syndrome (BWS). However, PMD is both underdiagnosed and underreported. Cases of PMD with gestational ages less than 20 weeks are rarely reported.

Design: In order to elucidate the clinical presentation, complications, microscopic features, and differential diagnoses, 12 cases of PMD with gestational ages less than 20 weeks were clinicopathologically analyzed. An immunohistochemical study of p57 (Kip2) (p57) and TSSC3, both of which are products of paternally imprinted, maternally expressed genes, was also done.

Results: The patients' ages ranged from 23 to 40 years. Five patients each were initially diagnosed as PM or missed abortion, and 2 were diagnosed as CM with twin on ultrasound examinations. The gestational ages ranged from 7 to 19 weeks. One case was associated with BWS. In 10 cases, early stage PMD was histologically characterized by moderate swelling of stem villi with cistern formation, myxoid change, dilated veins, stromal cell proliferation, and the absence of trophoblastic hyperplasia. No dilated subchorionic vascular vessels with or without luminal thrombosis, or chorangiosis, which are observed in 3rd-trimester cases of PMD, were found. In all cases, cytotrophoblasts were positive for $\mathrm{p} 57$ and TSSC 3 . Regarding p57, villous stromal cells were diffusely positive in 3 cases, focally positive in 5 , and uniformly negative in 2. The remaining 2 cases were diagnosed as PMD with $\mathrm{CM}$, and the stromal cells of PMD component were negative for $\mathrm{p} 57$

Conclusions: PMD presents with a wide spectrum of clinicopathologic findings. Early stage PMD can be clinically or pathologically misdiagnosed as missed abortion, PM or $\mathrm{CM}$ with twin. Two cases were diagnosed as PMD with $\mathrm{CM}$. The histology of early stage PMD are less distinctive than those of PMD in the 3rd trimester. The diagnostic clues that can be used to identify such cases include moderate swelling of stem villi with cistern formation, myxoid change, dilated veins, mild stromal cell proliferations, and the absence of trophoblastic hyperplasia. Immunohistochemical examinations of p57 expression are useful for differential diagnosis in equivocal cases. It is important to identify PMD at an early stage to reduce the risk of fetal morbidity or mortality. Patients with PMD with CM should be followed-up with HCG monitoring.

1146 Complete Hydatidiform Moles with Mosaic Histology: Histological and Immunohistochemical Studies of Ten Cases

Masaharu Fukunaga. Shin-Yurigaoka General Hospital, Kawasaki, Japan.

Background: Complete moles (CM) with mosaic histology are very rare, and they may pose a diagnostic challenge. Microscopically, they resemble a twin placenta with $\mathrm{CM}$ or partial mole (PM). In such cases, differential diagnosis is very important for patient management.

Design: Ten cases of CM with mosaic histology, all of which were in the first trimester, were retrieved, and their initial diagnoses included CM (3 cases), a twin placenta with CM (2), CM with mosaic histology (2), PM (2), and hydropic abortion with focal trophoblastic hyperplasia (1). They were histopathologically analyzed with immunostaining of $\mathrm{p} 57$ (Kip2) (p57) and TSSC3, which are products of paternally imprinted, maternally expressed genes.

Results: The patients' ages ranged from 20-36 years (mean, 31 years), and the gestational ages ranged from 7 to 13 weeks (mean, 9.0 weeks). Histologically, 5 cases had both $\mathrm{CM}$ and placental mesenchymal dysplasia (PMD) components, in which villous stromal cell proliferation and the absence of trophoblastic hyperplasia were observed. The 5 remaining cases had $\mathrm{CM}$ and non-PMD components, in which almost normal villi were observed. Cytotrophoblasts and stromal cells of CM components were negative for p57, and no cytotrophoblastic expression of TSSC3 was detected in any case. Cytotrophoblasts and villous stromal cells of non-PMD components expressed p57 and cytotrophoblasts were positive for TSSC3. In PMD components, cytotrophoblasts were positive for $\mathrm{p} 57$ and TSSC 3 , and villous stromal cells were negative for $\mathrm{p} 57$, indicating that the stromal cells were androgenetic, and the cytotrophoblasts were biparental. Fetus parts were observed in two cases. CM with mosaic histology could be distinguished from $\mathrm{CM}$ with twin and PM based on the lack of two populations of molar and non-molar villi, and the presence of mosaic villi. None of the patients developed persistent disease. Conclusions: The findings support the hypothesis that the misexpression of p57 and TSSC3 is involved in the abnormal development of androgenic CM. CM with mosaic histology can be classified into two groups; CM with PMD components and $\mathrm{CM}$ with non-PMD components. None of the cases was $\mathrm{CM}$ with twin, but rather $\mathrm{CM}$ with androgenic/biparental chimera or mosaic molar gestation and PMD. The villous histology and immunohistochemical profiles were correlated. Immunohistochemistry of the imprint gene products $\mathrm{p} 57$ and TSSC 3 can be a useful screening tool for cytogenetic analyses of $\mathrm{CM}$ with mosaic histology. None of the patients with $\mathrm{CM}$ with mosaic histology developed persistent disease.

1147 Clinicopathological and Immunohistochemical Studies of Twin Placenta with Complete Hydatidiform Mole

Masaharu Fukunaga. Shin-Yurigaoka General Hospital, Kawasaki, Japan.

Background: Twin pregnancies involving a normal placenta and complete mole $(\mathrm{CM})$ carry a higher risk of persistent trophoblastic disease than conventional CM and should be differentiated from its mimics, such as $\mathrm{CM}$ with mosaicism and partial moles (PM) to facilitate patient management.

Design: Twelve cases of twin pregnancies with $\mathrm{CM}$ in the first trimester and 3 cases of clinically putative twin pregnancies with $\mathrm{CM}$ were investigated by immunostaining p57 (Kip2) (p57) and TSSC3, which are products of paternally-imprinted, maternallyexpressed genes. DNA ploidy was also analyzed by flow cytometry in 3 cases, all of which were diploid. One case was chromosomally analyzed.

Results: The patients' ages ranged from 20-38 years (mean, 33 years), and the gestational ages ranged from 8 to 31 weeks (mean, 11.5 weeks). Clinically, hydatidiform mole was suspected in 10 cases, and missed abortion or blighted ovum was suspected in 5 cases. The initial pathological diagnosis was $\mathrm{CM}$ in 9 cases and PM in 6 cases. On review, 12 cases were histologically diagnosed as $\mathrm{CM}$ with twins (a twin pregnancy with a coexisting normal placenta and $\mathrm{CM}$ ). The admixture of large hydropic villi with circumscribed trophoblastic hyperplasia and smaller, normal-appearing villi without trophoblastic hyperplasia was observed in all cases. The cytotrophoblasts and stromal cells in the larger villi were negative for p57 (androgenic) and TSCC3, whereas these cells were positive in the smaller villi (biparental). Two cases were CM with foci of placental mesenchymal dysplasia (PMD). In the remaining case, in which the larger villi exhibited a 46XX karyotype, and the normal-appearing villi displayed a 46XY karyotype, the cytotrophoblasts in the larger villi were focally positive for TSSC3 but uniformly negative for $\mathrm{p} 57$. The normal-appearing villi exhibited p57 and TSSC3 expression in cytotrophoblasts. Two patients delivered live babies, and 2 patients with $\mathrm{CM}$ with twins developed lung metastasis.

Conclusions: Immunohistochemical analysis is a useful tool for the differential diagnosis of twin placenta with CM and morphologically similar conditions. In the present study, 2 cases were not twins with CM, but rather were diagnosed as CM with an androgenic/biparental chimera or mosaic molar gestation and PMD. One case of twins with a $\mathrm{CM}$ was suspected to have been caused by an androgenic/biparental chimera or mosaic molar and normal-appearing villi, although no molecular cytogenetic analysis was performed. Immunohistochemical staining of the imprint gene products p57 and TSSC 3 might be a useful screening tool for cytogenetic analyses. 
1148 Molecular Modifiers of Hormone Receptor Action: Decreased Androgen Receptor Expression in Mismatch Repair Deficient Endometrial Endometrioid Adenocarcinoma

Qiong Gan, Suzanne Crumley, Russell R Broaddus. MD Anderson Cancer Center, Houston, TX.

Background: Estrogen and progesterone receptors have traditionally been the most intensely studied hormone receptors in endometrial endometrioid adenocarcinoma (EEC). There is evidence that androgen signaling also plays a role in EEC pathogenesis. Endometrial cancer risk is tightly linked to obesity, and obesity is associated with a hyperandrogenic state. Polycystic ovarian syndrome associated with androgen excess also increases the risk of EEC. Recently, the androgen receptor (AR) antagonist Enzalutamide has been introduced as a therapeutic alternative for hormone receptor positive cancers, and clinical trials for gynecological cancers are underway. Factors impacting AR expression in EEC are not well understood. Mismatch repair (MMR) deficiency is one of the most common molecular alterations in EEC, occurring in 15$20 \%$ of cases. The aim of this study was to determine if AR expression is altered by MMR deficiency.

Design: 336 EECs were screened for MMR deficiency using immunohistochemistry (IHC) for MLH1, MSH2, MSH6, and PMS2. Cases with loss of MLH1 expression were tested for $M L H 1$ methylation. To minimize effects of patient age, tumor histotype and grade, AR expression was studied in age-matched grade $2 \mathrm{EEC}$, MMR-intact $(\mathrm{n}=49)$ and MMR-deficient due to $M L H 1$ methylation ( $\mathrm{n}=47$ ). AR expression was evaluated using both a clinically validated cut-off established by ASCO/CAP for ER and PR in breast cancer and an Allred scoring system. In this system, the proportion of positive staining is scored on a scale of $0-5$ and staining intensity is on a scale of 0-3. The final score adds both together to yield a total score. A score of 0-2 was regarded as negative and 3-8 as positive. Data were analyzed using the Fisher exact test and Chi-Square analysis. Results: Among all cases, AR was positive in 74\% (CAP) and $60 \%$ (Allred). In the MMR-intact group, $82 \%$ (CAP) and $69 \%$ (Allred) were AR positive. In the MMRdeficient group, AR positivity was significantly lower at $66 \%$ (CAP) and $49 \%$ (Allred) compared to the MMR-intact group $(\mathrm{p}<0.05)$. Moreover, the average AR score, calculated by adding percentage score and intensity score, was significantly higher in MMR-intact (3.5) compared to MMR-deficient (2.7; $\mathrm{p}=0.047)$. PTEN IHC loss, another common finding in EEC, had no impact on AR positivity.

Conclusions: This is the first report of AR expression in EEC with known MMR status. The results show that AR expression is negatively correlated with MMR deficiency. This should be taken into account when designing androgen blockade clinical trials.

1149 Molecular Modifiers of the Immune Microenvironment in Endometrial Endometrioid Adenocarcinoma: PTEN Loss and MMR Deficiency Are Associated with Increased Expression of the Immunosuppressive Signaling Protein STAT6

Qiong Gan, Suzanne Crumley, Russell R Broaddus. MD Anderson Cancer Center, Houston, TX.

Background: Mismatch repair (MMR) deficiency and PTEN loss are two of the most common molecular events in endometrial endometrioid adenocarcinomas (EEC). Both are associated with an immunosuppressive tumor microenvironment. For PTEN loss, this local immune suppression has recently been demonstrated in melanoma. The molecular basis for the immune modifying effects of MMR deficiency and PTEN loss are not known. The STAT6 signaling pathway is activated by IL-4 and IL-13 signaling, upregulates the immune checkpoint ligand PD-L2, and thus suppresses immune responses against cancer. The aim of this study was to determine the impact of MMR and PTEN on the expression of STAT6 in EEC.

Design: 336 EECs were screened for MMR deficiency using immunohistochemistry (IHC) for MLH1, MSH2, MSH6, and PMS2. Cases with loss of MLH1 expression were tested for $M L H 1$ methylation. To minimize effects of patient age and tumor histotype and grade, STAT6 expression was studied in age-matched grade 2 EEC, MMR-intact $(\mathrm{n}=49)$ and MMR-deficient due to $M L H 1$ methylation $(\mathrm{n}=47)$. PTEN status was assessed by IHC. STAT6 IHC expression was evaluated using an Allred scoring system. In this system, the proportion of positive staining was scored on a scale of $0-5$, and staining intensity on a scale of $0-3$. A score of $0-2$ was regarded as negative, while 3-8 as positive. The data were analyzed using the Fisher exact test and Chi-Square analysis to compare STAT6 expression by MMR and PTEN status.

Results: Overall, cytoplasmic STAT6 expression was observed in $58 \%$ of grade 2 EEC. In the MMR-deficient group, $72 \%$ of cases were positive for STAT6, with the positivity rate significantly decreased in the MMR-intact group $(45 \%$; $<0.05)$. The average STAT6 score in the MMR-deficient group was 3.8, significantly higher than in the MMR-intact tumors $(2.4 ; \mathrm{p}<0.05)$. STAT6 expression was also positively correlated to PTEN loss, so that if PTEN expression was lost, the tumor was more likely to express STAT6 $(\mathrm{P}<0.05)$ Conclusions: Both MMR deficiency and PTEN loss are positively correlated with STAT6 expression in EEC. The mechanism of STAT6 signaling in EEC is unknown. It is known that the majority of the STAT6 cellular pool is cytoplasmic and that nongenomic, non-nuclear signaling by STAT6 has been reported in other systems. These data highlight how important molecular mechanisms in EEC pathogenesis, such as PTEN and MMR, may potentially impact therapeutic approaches using checkpoint inhibition.

1150 Expression of SALL4 in Undifferentiated Endometrial Carcinoma and Correlation with Outcome

Qiong Gan, Preetha Ramalingam. MD Anderson Cancer Center, Houston, TX. Background: SALL4 initially identified as a sensitive and specific marker for germ cell tumors (GCT) is also expressed in a variety of carcinomas including undifferentiated carcinomas (UCA) of the esophagus and sinonasal tract. SALL4 expression in endometrial UCA (UCAe) has not been well studied. SALL4 is known to downregulate
PTEN expression in hematopoietic tumors. Loss of PTEN has been demonstrated in UCAe but it's correlation with SALL4 has not been reported. The aim of this study is to examine SALL4 and PTEN expression in UCAe to determine if there is correlation between these two markers and outcome.

Design: 23 UCAe cases (16 dedifferentiated carcinoma (DDCe) and 7 pure UCAe) were stained with SALL4 and PTEN. Nuclear staining of SALL4 in $>5 \%$ of tumor cells was considered positive. More than $50 \%$ of PTEN loss within the tumor cells in areas with intact internal control (stromal cells) was defined as PTEN loss. Correlation of expression patterns with outcome was evaluated. Data was analyzed using Fisher exact test and Chi-Square analysis.

Results: 16 were DDCe and 7 pure UCAe. Age range in pure UCAe was 50-70 yrs (mean 61.5) and 46-79 $\mathrm{yrs}(\mathrm{mean} 62.5)$ in DDCe ( $\mathrm{p}=0.83)$. SALL4 was positive in 11 of $23(48 \%)$ UCAe cases. Staining was present only in the UCA component and not in the low grade carcinoma in DDCe cases. SALL4 was positive in 6 of 7 pure UCAe $(86 \%)$ and 5 of $13(31 \%)$ DDCe $(\mathrm{p}=0.016)$. Staining ranged from $8-60 \%$ (mean 37\%). PTEN was lost in 15 of $23(65 \%)$ UCAe (5/7 (71\%) pure UCAe and 10/16 (63\%) DDCe). Correlation between PTEN and SALL4 expression is in Table 1.

\begin{tabular}{|l|l|l|l|l|l|}
\hline & \multicolumn{2}{|c|}{ DDC } & \multicolumn{2}{c|}{ Pure UCA } & \multicolumn{1}{c|}{ Total } \\
\hline & SALL4+ & SALL4- & SALL4+ & SALL4- & \\
\hline PTEN loss & 3 & 7 & 4 & 1 & $15(65 \%)$ \\
\hline PTEN retained & 2 & 4 & 2 & 0 & $8(35 \%)$ \\
\hline Total & 5 & 11 & 6 & 1 & 23 \\
\hline
\end{tabular}

Follow-up was available in 20 cases. SALL4 expression appears to be associated with better survival but was not statistically significant $(\mathrm{p}=0.18)$; Table 2 .

\begin{tabular}{|l|l|l|l|l|}
\hline & DOD & ANED & AWD & Total \\
\hline SALL4+ & $3(33 \%)$ & $5(56 \%)$ & $1(11 \%)$ & 9 \\
\hline SALL4- & $7(64 \%)$ & $3(27 \%)$ & $1(9 \%)$ & 11 \\
\hline PTEN+ & $6(46 \%)$ & $5(39 \%)$ & $2(15 \%)$ & 13 \\
\hline PTEN- & $4(57 \%)$ & $3(43 \%)$ & 0 & 7 \\
\hline
\end{tabular}

DOD: Died of diease (dis) ANED: Alive no evidence of dis, AWD: Alive with dis. Conclusions: SALL4 is expressed in majority of pure UCAe and cannot be used to differentiate them from GCT. PTEN is lost in $>60 \%$ of pure UCA and DDC and likely plays a role in their pathogenesis. There is no correlation between SALL4 and PTEN expression. SALL4 expression appears to correlate with better overall survival but larger studies are needed to confirm this finding.

1151 Immunohistochemical Expression of p53 and WT1 Is Retained Following Chemotherapy in High-Grade Serous Tubo-Ovarian Carcinoma (HGSC)

Raji Ganesan, Naveena Singh, Laura Casey, Simone Tam, Asma Faruqi, Rajeev Prasad, Steffen Boehm, Michelle Lockley, Arjun J Jeyarajah, Blake Gilks, Martin Koebel. Birmingham Womens Hospital NHS Foundation Trust, Birmingham, United Kingdom; Barts Health NHS Trust, London, United Kingdom; Queen's Hospital, Romford, Essex, United Kingdom; Queen Mary University of London, London, United Kingdom; Vancouver General Hospital, Vancouver, BC, Canada; University of Calgary, Calgary, AB, Canada.

Background: Treatment of HGSC patients is increasingly based small biopsy samples and the first surgical resection specimen often follows chemotherapy. p53 and WT1 are important diagnostic markers of HGSC. The effect of chemotherapy on p53 and WT1 expression has not been widely studied.

We aimed to compare p53 and WT1 expression in paired pre- and post-chemotherapy HGSC samples.

Design: Paired omental samples of HGSC pre- and post chemotherapy were obtained. Immunohistochemistry (IHC) was carried out for p53 and WT1. p53 staining was recorded as wild type or abnormal (overexpression (OE), complete absence (CA) or cytoplasmic (CY)), and WT1 as positive or negative (Table 1).

\begin{tabular}{|l|l|}
\hline p53 WT & Variable nuclear staining, weak to moderate in $1-80 \%$ tumour nuclei \\
\hline p53 abn-OE & Strong nuclear staining in $>80 \%$ of tumour cells \\
\hline p53 abn-CA & Complete absence of nuclear staining with intrinsic control present \\
\hline p53 abn-CY & Cytoplasmic staining without nuclear over expression \\
\hline WT1 positive & Nuclear staining in $>1 \%$ tumour cells \\
\hline
\end{tabular}

Results: 58 paired samples were included in the study. The number of chemotherapy cycles ranged from 3-6 (median 3). Pre- and post-chemotherapy p53 and WT1 IHC results (Table 2) were concordant in 55 of $58(95 \%)$ and 56 of $56(100 \%)$ respectively.

\begin{tabular}{|c|c|c|c|c|c|c|c|}
\hline & \multicolumn{7}{|c|}{ Pre-Chemo IHC expression } \\
\hline \multirow{7}{*}{$\begin{array}{c}\text { Post-Chemo } \\
\text { IHC } \\
\text { expression }\end{array}$} & & $\begin{array}{l}\text { p53 abn- } \\
\text { OE }\end{array}$ & $\begin{array}{l}\text { p53 abn- } \\
\text { CA }\end{array}$ & $\begin{array}{l}\text { p53 abn- } \\
\text { CY }\end{array}$ & $\begin{array}{l}\text { p53 } \\
\text { WT }\end{array}$ & $\begin{array}{l}\text { WT1 } \\
+\end{array}$ & WT1 \\
\hline & $\begin{array}{l}\text { p53 abn- } \\
\text { OE }\end{array}$ & $40^{*}$ & - & - & - & & \\
\hline & $\begin{array}{l}\text { p53 abn- } \\
\text { CA }\end{array}$ & - & 12 & - & - & & \\
\hline & $\begin{array}{l}\text { p53 abn- } \\
\text { CY }\end{array}$ & - & 1 & 2 & - & & \\
\hline & p53 WT & $1^{* *}$ & - & 1 & 1 & & \\
\hline & WT1 + & & & & & 56 & - \\
\hline & WT1 - & & & & & - & - \\
\hline
\end{tabular}

* One case showed a mixed pattern of $\mathrm{OE}$ and $\mathrm{CA}$

**Mosaic pattern of strong but variable staining 
Conclusions: Our results demonstrate that immunohistochemical expression of $\mathrm{p} 53$ and WT1 is retained following chemotherapy. The findings underscore firstly the reliability of these diagnostic markers in small samples and secondly that the pattern of expression is not altered by chemotherapy.

1152 Genomic Characterization of Histologically Distinct Components of Uterine Carcinosarcomas

Felipe Geyer, Kathleen A Burke, Charlotte K Ng, Ronglai Shen, Nicola Fusco, Caterina Marchio, Rajmohan Murali, Robert Soslow, Jorge Reis-Filho, Britta Weigelt. Memorial Sloan Kettering Cancer Center, New York, NY.

Background: Uterine carcinosarcomas (CSs) are biphasic lesions composed of high-grade carcinoma and sarcoma elements. Current data based on X-chromosome inactivation, loss of heterozygosity $(\mathrm{LOH})$ and limited sequencing favor the notion that in the majority of CSs histologically distinct components within CSs are clonally related. Here we sought to characterize the epithelial and sarcoma components of CSs and to define the directionality of progression using whole-exome sequencing (WES) analysis. Design: The carcinoma and sarcoma components of 6 fresh-frozen CSs were separately microdissected. DNA extracted from each component and matched normal tissue was subjected to WES. 68 non-synonymous somatic mutations identified by WES were subjected to targeted amplicon re-sequencing, with a validation rate of $97.1 \%$. Somatic genetic alterations, cancer cell factions of mutations and mutational signatures were identified using state-of-the-art bioinformatics algorithms. Clonal decomposition was performed using mclust and SubcloneSeeker.

Results: Medians of $71(54-1,485)$ and $84(53-1,103)$ somatic mutations were identified per case in the carcinoma and sarcoma components, respectively. Both components of all but one CS displayed an aging mutational signature. A mismatch repair deficiency (MMR) mutational signature was found in the remaining CS, in which both components showed loss of immunohistochemical expression of MLH1 and PSM2, shared 90 mutations and similar simple copy number profiles. In the 5 MMR-proficient CSs, both components displayed similar gene copy number profiles and shared $56 \%$ to $93 \%$ of all mutations, including clonal mutations affecting known cancer genes, such as PIK3CA, $P T E N, R B 1, T P 53, K R A S$ and CHD4. Identical clonal TP53 mutations coupled with $\mathrm{LOH}$ were found in both components of $3 \mathrm{CSs}$. No recurrent mutations restricted to either the carcinoma or the sarcoma components in the 5 MMR-proficient CSs were found. Clonality and clonal decomposition analyses confirmed the clonal relatedness between components in all CSs, and in 2 cases provided strong circumstantial evidence supportive of genetic progression from the carcinoma to the sarcoma component. Conclusions: Our findings demonstrate that most uterine CSs are monoclonal in origin, that MMR deficiency and TP53 inactivation precedes the emergence of the distinct components, and corroborate the notion that CSs are actually carcinomas that undergo mesenchymal metaplasia, possibly through clonal evolution.

1153 Comparison of Clinicopathological Outcomes for Insufficient and Scant Endometrial Samples

Emily Goebel, Helen Ettler, Meg M McLachlin, Michele M Weir. Western University, London, ON, Canada.

Background: There are no defined pathologic criteria for reporting endometrial samples (ems) with limited tissue as either scant or insufficient. There is also no consensus on the clinical follow up of patients with these samples and whether terminology reflects outcome. The purpose of this study was to compare clinicopathological outcomes for scant and insufficient ems.

Design: A retrospective chart review was conducted on all patients with insufficient or scant endometrial biopsies or curettings from 2010-13, at our center. Clinicopathological features of the patients were recorded including age, presenting symptoms, repeat sampling, hysterectomy, non-surgical management and final pathology diagnosis. Five gynecologists were surveyed using an anonymous questionnaire about their practice for managing patients with these samples.

Results: Table 1: Comparison of Outcomes of Patients with Insufficient and Scant Endometrial Samples.

\begin{tabular}{|l|c|c|c|}
\hline $\begin{array}{l}\text { Endometrial Sample Types* } \\
(\mathrm{n}=1149)\end{array}$ & $\begin{array}{c}\text { Insufficient } \\
(49 \%)\end{array}$ & $\begin{array}{c}\text { Scant } \\
(51 \%)\end{array}$ & P value \\
\hline Repeat ems & $33 \%$ & $31 \%$ & 0.33 \\
\hline Hyperplasia on repeat ems & $9.6 \%$ & $8 \%$ & 0.7 \\
\hline Malignancy on repeat ems & $7 \%$ & $2.8 \%$ & 0.07 \\
\hline Subsequent Surgery & $16 \%$ & $11 \%$ & 0.02 \\
\hline Repeat ems prior to surgery & $45 \%$ & $37.5 \%$ & 0.36 \\
\hline Malignancy on hysterectomy & $19 \%$ & $9 \%$ & 0.10 \\
\hline
\end{tabular}

*ems: $89 \%$ biopsies, $11 \%$ curettings

In the survey of gynecologists, $4 / 5$ reported that they managed patients with scant or insufficient biopsies/curettings similarly.

Conclusions: The similar repeat ems rate between scant and insufficient samples suggests that our clinicians are choosing similar management for both of these terminologies. This finding is in keeping with the results of the questionnaire. Given the malignancy outcomes, both insufficient and scant ems merit repeat patient sampling in the appropriate context. As a follow-up to this study, our center has conducted a review of scant and insufficient ems to determine pathologic criteria for reporting.
1154 Insufficient and Scant Endometrial Samples: Determining Pathological Criteria and Consistency in Reporting

Emily Goebel, Meg M McLachlin, Helen Ettler, Michele M Weir. Western University, London, ON, Canada.

Background: Scant and insufficient endometrial biopsies are terms used for endometrial samples with minimal or no tissue. Although some criteria have been suggested, there are no established guidelines for determining endometrial sample adequacy. The objective of this study was to determine criteria and consistency for reporting insufficient and scant endometrial samples.

Design: 99 cases of previously reported scant or insufficient endometrial samples were reviewed blindly and separately by 3 pathologists and one resident. Several qualifiers were recorded including number of endometrial epithelial strips (EES) and stromal fragments (SF). Each reviewer assigned the cases as scant, insufficient or diagnostic. Results were compared across reviewers.

Results: Table 1: Agreement in Reporting Endometrial Samples

\begin{tabular}{|l|l|l|l|l|}
\hline $\begin{array}{l}\text { Agreement Across } \\
\text { Reviewers*N=99 }\end{array}$ & Insufficient & Scant & Diagnostic & $\begin{array}{l}\text { Overall \% oftotal } \\
\text { cases }\end{array}$ \\
\hline $100 \%$ agreement & $45 \%$ & 0 & $11 \%$ & $57 \%$ \\
\hline $75 \%$ agreement & $8 \%$ & $4 \%$ & $6 \%$ & $18 \%$ \\
\hline
\end{tabular}

*Fleiss $\kappa=0.49$ (standard error 0.03 ; $95 \%$ CI 0.43 to 0.55 )

Threshold for reporting a sample as diagnostic varied with $2 / 4$ reviewers requiring SF (1-2 for one and 3-5 for another when EES were absent). When no SF were present, one reviewer required at least 6-10 EES, while the other required $>10$ for a diagnostic sample. There was no consensus in reporting a sample as insufficient with criteria ranging from 0 to $10 \mathrm{EES}$ without SF to 3-5 SF when 1-2 EES were present.

Conclusions: There was moderate agreement between reviewers when reporting endometrial samples as insufficient, scant or diagnostic based on Fleiss Kappa. Insufficient samples with no identified EES or SF had the highest agreement. The thresholds for reporting a specimen as diagnostic varied across reviewers from at least $6 \mathrm{EES}$ to the requirement of SF. Consensus meeting and standardization are required to ensure consistency in reporting.

1155 PD-L1 Expression in Tumor-Associated Macrophages in Primary and Metastatic High-Grade Serous Ovarian Carcinoma

Chelsea Gottlieb, Kari Ring, Anne Mills. University of Virginia, Charlottesville, VA. Background: The role of PD-L1/PD-1 inhibitor therapies in the treatment of ovarian high grade serous carcinoma (HGSC) is still being established. Data on PD-L1 expression in ovarian HGSC is mixed, with some studies reporting robust tumor staining (particularly in BRCA-mutated cancers) and others identifying expression limited to tumor-associated macrophages (TAM). There is evidence that TAM PD-L1 expression is induced in metastases/implants from patients who have undergone chemotherapy, as neoadjuvant treatment is thought to modulate the immune microenvironment of ovarian HGSC. However, it is unclear whether TAM acquisition of PD-L1 plays a role in tumor spread in the absence of prior treatment. We investigated PD-L1 expression in primary ovarian tumors and matched metastatic implants from predominantly treatment-naïve HGSC.

Design: 61 primary HGSC ( 44 treatment-naïve, 13 post-neoadjuvant, 4 treatment status unknown) were evaluated with PD-L1 and CD68 IHC: 40 on TMA ( $0.6 \mathrm{~mm}$ cores, 4 cores/case) and 21 on whole section. Whole section cases were matched to a metastasis/ omental implant. 16 matched cases were treatment-naïve in both specimens and none had undergone chemotherapy between the primary and metastatic specimens. TAMs were delineated by $\mathrm{CD} 68$ and classified as focal, moderate, or brisk. Membranous PDL1 staining was scored separately for tumor cells and TAM by \%.

Results: $8 \%$ of primary HGSC showed tumor staining for PD-L1; staining was focal in all but 1. In contrast, $74 \%$ showed PD-L1+ TAMs. Patients with known $B R C A$ mutations were more likely to be PD-L1+ in tumors $(40 \% B R C A+$ vs. $0 \% B R C A-, p=0.05)$. Cases that had undergone neoadjuvant therapy were more likely to show PD-L1+ TAM $(78 \%$ vs. $34 \%, p=0.01$ ) but had comparable rates of primary tumor staining. Most cases showed consistent staining across primary/metastasis for both tumor (76\%) and TAM $(95 \%)$, and prior therapy did not significantly influence these results.

Conclusions: PD-L1+ TAM are common in both primary and metastatic ovarian HGSC, however tumoral PD-L1 staining is rare except in BRCA-mutated cancers. While PD-L1+ TAM are more common in both primaries and metastases after neoadjuvant therapy, PD-L1 gain does not appear to play a role in metastasis in treatment-naive cancers. Clinical trials are needed to determine whether TAM staining correlates with clinical response to PD-1/PD-L1 inhibition.

\section{Detection of Incidental Lobular Endocervical Glandular Hyperplasia in Hysterectomy Specimens Removed for Non-Cervical Lesions}

Sultan A Halimi, Daichi Maeda, Akiteru Goto. Akita University, Akita, Japan; Kabul Medical University, Kabul, Afghanistan.

Background: Lobular endocervical glandular hyperplasia (LEGH) is a uterine cervical lesion composed of gastric-type glands without atypia. LEGH is now recognized as a precursor of gastric-type mucinous carcinoma, a subtype of endocervical adenocarcinoma known for its poor prognosis. In general, the clinical detection of LEGH is difficult, and its accurate pathological diagnosis may require expertise and special staining methods. To date, no study has investigated the prevalence of LEGH in detail and, thus, it is difficult to estimate how often LEGH undergoes malignant transformation. Design: A total of 350 consecutive hysterectomy specimens with cervical sections were archived from the Department of Pathology at the Akita University Hospital. In 301 cases, hysterectomy was performed for non-cervical lesions. The remaining (49/350) 
patients had cervical lesions diagnosed preoperatively. Hematoxylin and eosin-stained slides were reviewed for LEGH by a gynecological pathologist. To confirm the diagnosis of LEGH, Alcian blue-PAS double staining and immunohistochemistry for Claudin-18, HIK 1083 and ER were performed.

Results: In total, five LEGHs were found. One was observed in the background of a cervical gastric-type mucinous carcinoma. Four were incidental (clinically occult) LEGHs that were identified in hysterectomy specimens submitted for non-cervical lesions $(4 / 301,1.3 \%)$. Clinicopathological features of these incidental LEGHs are summarized in Table 1. All the LEGHs contained neutral mucin, and were immunoreactive for a gastric marker Claudin-18. At least focal immunoreactivity for HIK1083, another gastric marker, was observed in most of the LEGHs. The expression of ER was decreased or lost in all cases.

\begin{tabular}{|c|c|c|c|c|c|}
\hline Case & \multirow{2}{*}{ Age } & $\begin{array}{c}\text { Size of } \\
\text { incidental } \\
\text { LEGH }\end{array}$ & $\begin{array}{c}\text { Pathological diagnosis of the } \\
\text { main lesion }\end{array}$ & \multicolumn{2}{|c|}{ Preoperative cytology } \\
\cline { 5 - 6 } & & & Endocervix & Endometrium \\
\hline 1 & 48 & $16 \times 14 \mathrm{~mm}$ & $\begin{array}{c}\text { Ovarian high grade serous } \\
\text { carcinoma }\end{array}$ & Negative & Negative \\
\hline 2 & 42 & $6 \times 4 \mathrm{~mm}$ & $\begin{array}{c}\text { Uterine low grade endometrial } \\
\text { stromal sarcoma }\end{array}$ & Not done & Negative \\
\hline 3 & 40 & $9 \times 5 \mathrm{~mm}$ & Uterine adenomyosis & Negative & Negative \\
\hline 4 & 62 & $6 \times 2 \mathrm{~mm}$ & $\begin{array}{c}\text { Ovarian endometrioid } \\
\text { carcinoma }\end{array}$ & Not done & Not done \\
\hline
\end{tabular}

Conclusions: Clinically occult LEGH can be identified in more than $1 \%$ of hysterectomy specimens. Considering the low lifetime prevalence of cervical gastric-type mucinous carcinoma, which is estimated to be around $0.05 \%$ in the Japanese population, we speculate that most patients with LEGH follow a benign clinical course.

\section{Uterine Polyps in the Setting of Tamoxifen Use: An Institutional} Experience

Lisa Han, Ricardo R Lastra. University of Chicago, Chicago, IL.

Background: Tamoxifen exposure has been associated with endometrial polyps, endometrial hyperplasia and malignant endometrial neoplasms. Tamoxifen-associated endometrial and endocervical polyps (TP) are characterized by periglandular stromal condensation, epithelial and stromal proliferative activity, and epithelial metaplasia. Because there are concerning overlapping features between TPs and Müllerian adenosarcomas, literature has suggested the former may be a precursor lesion to the latter. Our study characterizes and provides clinicopathologically follow-up of TPs.

Design: Sixty-nine uterine polyps from 51 patients on tamoxifen therapy were selected from our institutional archives from 1992-2016 and reviewed by a gynecologic pathologist for the following morphological characteristics: intraglandular polypoid projections (IPP); phyllodes-like architecture (PLA); periglandular stromal condensation (PSC); mitotic count; epithelial metaplasia (squamous, eosinophilic syncytial, mucinous, clear cell); stromal cytologic atypia (SCA). Clinical presentation, polyp size, past medical history, duration of tamoxifen therapy, pathologic (subsequent biopsies and hysterectomy) and clinical follow-up were recorded for each case.

Results: The most common morphologic features in TP was IPP (38\%), followed by PSC (32\%), epithelial metaplasias (23\%), increased stromal mitotic activity $(6 \%)$ and SCA (4\%). Eight cases demonstrated hyalinized stroma with overlying clear cell change, features not previously described to be associated with TPs. More endometrial than endocervical polyps had features of tamoxifen use ( $62 \%$ vs $19 \%)$. Of the 35 patients with tissue specimen follow-up, 15 had no residual uterine polyp, 18 had subsequent benign polyps and 2 developed a malignancy.

\begin{tabular}{|l|l|l|}
\hline Follow-up diagnosis & Polyp features & $\begin{array}{l}\text { Time after polyp } \\
\text { diagnosis (months) }\end{array}$ \\
\hline $\begin{array}{l}\text { High grade endometrial } \\
\text { stromal sarcoma }\end{array}$ & $\begin{array}{l}\text { PLA } \\
\text { Focal PSC } \\
1 \text { mitosis/10 HPF }\end{array}$ & 24 \\
\hline $\begin{array}{l}\text { High grade serous } \\
\text { endometrial carcinoma }\end{array}$ & $\begin{array}{l}\text { PLA } \\
\text { IPP } \\
\text { Prominent PSC } \\
\text { Syncytial, mucinous and squamous } \\
\text { epithelial metaplasia }\end{array}$ & 72 \\
\hline
\end{tabular}

Conclusions: There are well established features of TPs, some of which overlap with malignant stromal lesions such as low grade adenosarcoma. In our study, uterine polyps, even with multiple and pronounced features associated with tamoxifen use, do not behave in a malignant fashion. Recognizing these features and inquiring about tamoxifen therapy is important before raising the diagnostic possibility of a low-grade adenosarcoma.

1158 Genomic Abnormalities in Invasive Endocervical Adenocarcinoma Correlate with Pattern of Invasion: Biologic and Clinical Implications

Anjelica Hodgson, Yutaka Amemiya, Arun Seth, Matthew Cesari, Bojana Djordjevic, Carlos Parra-Herran. Sunnybrook Health Sciences Centre - University of Toronto, Toronto, ON, Canada.

Background: The risk of nodal spread and recurrence in patients with invasive endocervical adenocarcinoma (EA) is associated with the pattern of invasion as outlined by the Pattern-Based Classification System (PBCS). Several clinically important mutations are prevalent in EA; for instance, KRAS mutations have been associated with disease relapse, whereas PIK3CA mutations have been linked to better treatment outcomes. We aimed to correlate known oncogene and tumor suppressor gene alterations with the invasive pattern of EA.
Design: 20 EA cases treated with cone and hysterectomy were selected and classified using the PBCS by two gynecologic pathologists and one pathologist in training. Sequencing was performed in formalin-fixed, paraffin-embedded samples using the Ion AmpliSeq Cancer Hotspot Panel v2 (ThermoFisher Scientific, Waltham MA) to survey hotspot regions of 50 oncogenes and tumor suppressor genes. Single nucleotide mutations and copy number variations were correlated with invasive pattern.

Results: Five (25\%), six (30\%) and nine (45\%) cases were classified as patterns A, B, and $\mathrm{C}$ respectively. Pattern $\mathrm{B}$ and $\mathrm{C}$ tumors had a higher average number of mutations per case (2.5 and 2.1 per case, respectively) than pattern A tumors (1.2 per case). Prevalent alterations are depicted in Table 1. COSMIC mutations in KRAS and PIK3CA, as well as $E R B B 2$ and $H N F 1 A$ copy number gains were found exclusively in destructively invasive tumors (pattern B or C). 4/6 (67\%) cases with ERBB2 gain had concurrent gains in TP53. Novel $R B$ and $M E T$ mutations were also identified.

\begin{tabular}{|c|c|c|c|c|}
\hline \multirow{2}{*}{ Gene } & \multicolumn{4}{|c|}{ Pattern } \\
\cline { 2 - 5 } & $\mathrm{A}(\mathrm{n}=5)$ & $\mathrm{B}(\mathrm{n}=6)$ & $\mathrm{C}(\mathrm{n}=9)$ & Total $(\mathrm{n}=20)$ \\
\hline KRAS & 0 & $4(67 \%)$ & $2(22 \%)$ & $6(30 \%)$ \\
\hline PIK3CA & 0 & $2(33 \%)$ & $4(44 \%)$ & $6(30 \%)$ \\
\hline RB & 0 & $1(17 \%)$ & $1(11 \%)$ & $2(10 \%)$ \\
\hline MET & $1(20 \%)$ & $2(33 \%)$ & 0 & $2(10 \%)$ \\
\hline ERBB2 & 0 & $2(33 \%)$ & $4(44 \%)$ & $6(30 \%)$ \\
\hline TP53 & 0 & $1(17 \%)$ & $3(33 \%)$ & $4(20 \%)$ \\
\hline HNF1A & 0 & 0 & $2(22 \%)$ & $2(10 \%)$ \\
\hline
\end{tabular}

Conclusions: $K R A S, P I K 3 C A$ and $R B$ mutations, as well as ERBB2, TP53, and HNF1A gains are prevalent in EA and highly associated with destructive patterns of invasion. Novel mutations in $R B$ and $M E T$ were identified, which require further validation. Our findings indicate that the PBCS may serve as a triage tool for molecular testing in EA for prognostic and therapeutic purposes. Moreover, they suggest that non-destructive EA (pattern A) is biologically distinct from destructive EA, and that pattern B EA is more closely related to pattern C EA.

1159 High Grade Mullerian Adenosarcoma: Genomic and Clinicopathologic Characterization Indicative of a Distinct Neoplasm with Aggressive Behavior

Anjelica Hodgson, Yutaka Amemiya, Arun Seth, Bojana Djordjevic, Carlos ParraHerran. Sunnybrook Health Sciences Centre - University of Toronto, Toronto, ON, Canada.

Background: Mullerian adenosarcoma (AS) is an infrequent gynecologic malignancy. Sarcomatous overgrowth (SO), defined as pure sarcoma representing $\geq 25 \%$ of the tumor, usually confers a poor prognosis. Most AS are low grade (LG), but a subset of tumors show a high grade (HG) component. We hypothesized that HGAS has distinct clinical and molecular features, distinct from LGAS and independent from SO.

Design: Nine AS with a HG component were included. Clinical-pathologic features and follow-up data were recorded. Genomic analysis was carried out on FFPE tissue using the Ion AmpliSeq ${ }^{\mathrm{TM}}$ Comprehensive Cancer Panel targeting exons of 409 oncogenes and tumor suppressor genes (ThermoFisher Scientific, Waltham MA). Samples were composed entirely of HG tumor in all cases; in one case, the LG component was separately sequenced. Single nucleotide mutations, copy number gains $(\mathrm{CNG})>4$, and homozygous copy number losses (CNL) were analyzed.

Results: Mean patient age was 57 years (range 42-76). 8 HGAS tumors ( $89 \%$ ) had SO; $4(44 \%)$ had rhabdomyosarcomatous differentiation. The $\mathrm{HG}$ component comprised $\geq 75 \%$ of the tumor in $7 / 9$ cases; however, in $2 / 9$ cases it was $<25 \%$. Median tumor size was $12 \mathrm{~cm}$ (range 4.5-24 cm). At presentation, 33\% HGAS had extra-uterine disease. Follow-up was available in 7 patients (mean interval 11 months, range 2-25); $5(72 \%)$ had widespread local and distant recurrence. TP53 alterations were identified in 5 tumors $(56 \%)$ : either missense mutation $(\mathrm{n}=3)$, nonsense mutation $(\mathrm{n}=1)$ or $\mathrm{CNL}$ $(\mathrm{n}=1)$. Other frequent mutations involved $K M T 2 C(\mathrm{n}=8), K D M 5 C(\mathrm{n}=8), \operatorname{PTEN}(\mathrm{n}=3)$, $A T M(\mathrm{n}=3)$ and $P I K 3 C 2 B(\mathrm{n}=2) . C N G$ were seen in 7 cases $(78 \%)$; the most common genes, amplified in 2 cases each, were CDK4, MDM2, ITGA10, NBPF10, MIR6759, RNF213, EXT1, BCL6, LOC100131, LPP, PIK3CA and SOX-2. One case had only a minor $(10 \%) \mathrm{HG}$ component and no SO; however, the patient developed widespread recurrence of HG sarcoma 22 months after hysterectomy. Separate sequencing of HG and $\mathrm{LG}$ areas in this case revealed CNG in $B C L 6, L P P, P I K 3 C A$ and $S O X-2$ exclusively in the HG component and $J A K 3$ onlyin the LG component. Tumor also showed BAPI/ PBRMI loss in both areas.

Conclusions: HGAS is an aggressive tumor. TP53, KTM2C and KDM5C abnormalities are frequent in HGAS, which contrasts with previously reported rates in LGAS and AS with SO. In addition, HGAS has higher rates of CNG compared to the LG component. Although often associated with SO, a HG component in AS may constitute the driver of aggressive behavior even in the absence of SO, and should thus be reported.

1160 Fallopian Tube Examination: A Potential Strategy to Improve the Accuracy of Intraoperative Primary Site Assignment of Mucinous Tumors Involving the Ovary

Anjelica Hodgson, Sharon Nofech-Mozes, Dina Bassiouny, Fang-I Lu, Matthew Cesari, Nadia Ismiil, Carlos Parra-Herran, Bojana Djordjevic. Sunnybrook Health Sciences Centre - University of Toronto, Toronto, ON, Canada.

Background: Assessment of ovarian masses with mucinous neoplasms at frozen section (FS) remains a diagnostic challenge. The aim of this study was to determine whether FS examination of the FT improves the accuracy of classification of mucinous carcinomas involving the ovary as primary or metastatic. 
Design: 41 mucinous carcinomas involving the ovary (16 colonic, 5 appendiceal, 20 primary ovarian) were retrieved from our institutional research database. The original FS diagnosis of the ovarian mass (by a GYN or non-GYN pathologist) and a second review FS diagnosis (by a GYN pathologist blinded to all case information) were classified as 'primary', 'metastasis' or 'defer'. Permanent sections of FTs were examined and laterality, topography and pattern of metastases were recorded.

Results: FT metastases were present in $44 \%$ of colonic, $80 \%$ of appendiceal and $0 \%$ of ovarian primaries. FT metastases were either ipsilateral or bilateral (73\%) or contralateral (27\%) to the ovary examined on FS. $36 \%, 64 \%$ and $46 \%$ of FT metastases involved the fimbria, serosa and the wall, respectively, with no lesions in the lumen, mucosa or lymphovascular spaces. Colon metastases involved single FT sites, while $75 \%$ of the appendiceal metastases involved multiple FT sites. All FT metastases had an infiltrative pattern. Original FS classified only $33 \%$ of metastases and $55 \%$ primaries correctly. Review FS classified $81 \%$ of metastases and $70 \%$ primaries correctly. Detection of FT metastases in the ipsilateral FT would have correctly categorized metastatic tumors that were misclassified or deferred in the original FS by $36 \%$ and in the review FS by $25 \%$.

\begin{tabular}{|c|c|c|c|c|}
\hline & \multicolumn{2}{|c|}{ All Metastases, $\mathrm{n}=21$} & \multicolumn{2}{c|}{ Primary Ovarian, $\mathrm{n}=20$} \\
\hline & Original FS & $\begin{array}{c}\text { Review } \\
\mathrm{FS}\end{array}$ & Original FS & $\begin{array}{c}\text { Review } \\
\text { FS }\end{array}$ \\
\hline Cases classified correctly (\#,\%) & $7(33)$ & $17(81)$ & $11(55)$ & $14(70)$ \\
\hline Subset with FT invasion* (\#, sub \%) & $3(43)$ & $7(41)$ & 0 & 0 \\
\hline Cases misclassified (\#,\%) & $6(29)$ & $3(14)$ & 0 & $1(5)$ \\
\hline Subset with FT invasion* (\#, sub \%) & $2(33)$ & $1(33)$ & 0 & 0 \\
\hline Cases deferred (\#,\%) & $8(38)$ & $1(5)$ & $9(45)$ & $5(25)$ \\
\hline Subset with FT invasion* $(\#$, sub \%) & $3(38)$ & 0 & 0 & 0 \\
\hline
\end{tabular}

*Ipsilateral or bilateral FT invasion

Conclusions: Concurrent examination of ovary and ipsilateral FT at FS may significantly improve the accuracy of primary site assignment of mucinous neoplasms involving the ovary, even when the FS is performed by a specialist in GYN pathology. Metastases that involve multiple FT sites are preferentially associated with the appendix.

\section{Frozen Section Assessment of Mucinous Neoplasms Involving} the Ovary: A Novel Approach Toward a More Accurate Diagnosis

Anjelica Hodgson, Sharon Nofech-Mozes, Dina Bassiouny, Fang-I Lu, Matthew Cesari, Nadia Ismiil, Carlos Parra-Herran, Bojana Djordjevic. Sunnybrook Health Sciences Centre - University of Toronto, Toronto, ON, Canada.

Background: Assignment of primary vs. metastatic origin of mucinous tumors involving the ovary at frozen section (FS) is a common diagnostic challenge. As size and laterality are of limited value, the aim of this study was to identify tumor characteristics perceptible at FS that are most helpful in making this distinction.

Design: 41 mucinous carcinomas involving the ovary (16 colonic, 5 appendiceal, 20 primary ovarian) with FS material were retrieved from our institutional research database. Tumour laterality, ovarian size (larger ovary if bilateral), and original frozen section diagnosis (primary, metastasis or defer) were recorded. FS slides were examined for cytologic grade (low, focal high or diffuse high), tumor architecture (simple or complex glands, single cells/cell clusters), necrosis (none, luminal, diffuse), pattern of stromal invasion by tumor (none, expansile, focally infiltrative, diffusely infiltrative) and presence of stromal infiltrating mucin.

Results: Original FS classified $33 \%$ of metastases and $55 \%$ of primaries correctly $81 \%$ of metastases were $>13 \mathrm{~cm}$. Diffuse high grade cytology, diffusely infiltrative pattern of stromal invasion by tumor and stromal infiltrating mucin were exclusively seen in the metastases. Presence of any of these 3 features (even in cases with pure glandular architecture) identified metastases with $90 \%$ sensitivity, $100 \%$ specificity, $100 \%$ PPV and 91\% NPV. 1 colonic and 1 appendiceal metastasis were misclassified due to undersampling on FS.

\begin{tabular}{|c|c|c|c|}
\hline & $\begin{array}{c}\text { Metastases } \\
(\mathrm{n}=21)\end{array}$ & $\begin{array}{c}\text { Primary Ovarian } \\
(\mathrm{n}=20)\end{array}$ & P-value \\
\hline Tumor laterality - Unilateral (\#,\%) & 6,29 & 20,100 & 0.0001 \\
\hline Ovarian size (cm [range, median, mean] & $\begin{array}{c}4.5- \\
30,15.5,17.7\end{array}$ & $8-50,20.5,23.5$ & 0.04 \\
\hline Cytology - Diffuse high grade (\#,\%) & 9,43 & 0 & 0.001 \\
\hline $\begin{array}{c}\text { Tumor architecture - Single cells/clusters } \\
(\#, \%)\end{array}$ & 6,29 & 0 & 0.02 \\
\hline Necrosis - Diffuse (\#,\%) & 10,48 & 4,29 & 0.1 \\
\hline $\begin{array}{c}\text { Pattern of stromal invasion - Diffusely } \\
\text { infiltrative (\#,\%) }\end{array}$ & 11,52 & 0 & 0.0002 \\
\hline Stromal infiltrating mucin - Presence (\#,\%) & 7,33 & 0 & 0.009 \\
\hline
\end{tabular}

Conclusions: Ovarian metastases from colon and appendix commonly exceed $13 \mathrm{~cm}$ in size, a feature which may confound interpretation at FS, especially in unilateral or cases of unknown laterality. Metastatic tumors can be identified at FS by looking for diffuse high grade tumor cytology, diffusely infiltrative pattern of stromal invasion by tumor or stromal infiltrating mucin. Diagnosis of primary ovarian mucinous neoplasms is one of exclusion and should be made only after thorough sampling.
1162

Targeted Genomic Profiling of Female Adnexal Tumors of Probable Wolffian Origin

Brooke E Howitt, Elizabeth Garcia, Lynette M Sholl, Neal I Lindeman, Laura E MacConaill, Fei Dong, Michelle S Hirsch, Marisa R Nucci, Christopher P Crum, W Glenn McCluggage, Jelena Mirkovic. Brigham and Women's Hospital, Boston, MA; Harvard Medical School, Boston, MA; Dana-Farber Cancer Institute, Boston, MA; Belfast Health and Social Care Trust, Belfast, Northern Ireland, United Kingdom.

Background: Female adnexal tumor of probable Wolffian origin (FATWO) is a rare gynecologic neoplasm of low-malignant potential presumed to be derived from mesonephric remnants in the upper female genital tract. Similarly, mesonephric remnants of the lower female genital tract are thought to be the origin of mesonephric carcinoma (MCA). Although the molecular alterations in MCA have been recently reported, the pathogenesis of and molecular alterations in FATWO are not well understood. The aims of this study were to examine molecular alterations in FATWOs and to establish whether these are molecularly similar to those present in MCA.

Design: Eight FATWOs with available formalin-fixed paraffin embedded material were contributed by one of the authors (WGM). Genomic DNA was isolated from tumor samples and a targeted next generation sequencing assay was performed to detect single nucleotide variations (SNVs), copy number variations (CNVs), and structural variants by surveying exonic DNA sequences of 300 cancer genes and 113 introns across 35 genes. Results: Good quality DNA was isolated from 7 of 8 cases. Novel KMT2D mutations (1 frameshift, 3 missense) were identified in 4 of 7 cases ( $57 \%$ ). Mutations in chromatin remodeling genes (ARIDIA and ARIDIB) were identified in 2 of 7 cases (29\%). No cases harbored KRAS, NRAS, TP53, PIK3CA, PTEN, or DICERI mutations. There were relatively low numbers of CNVs, and no recurrent CNVs were identified. One case demonstrated high level amplification of $C C N D 1$. No structural variants were identified. Conclusions: FATWO is characterized by the absence of KRAS/NRAS mutations (characteristic of MCA), absence of DICERI mutations (characteristic of Sertoli-Leydig tumor) and frequent $K M T 2 D$ mutations. Loss of function mutations in $K M T 2 D$ (a histone methyltransferase involved in regulation of gene expression) have been described in a variety of tumors, however the novel missense mutations discovered in 3 of our cases are of uncertain biologic significance. In summary, FATWOs are characterized by a limited number of molecular aberrations that are significantly different from those reported in tumors in the differential diagnosis, and brings into question the relationship of MCA with FATWO.

1163 Targeted Genomic Profiling Reveals Recurrent KRAS Mutations and 1q Gain in Mesonephric Carcinoma-Like Adenocarcinomas of the Female Genital Tract

Brooke E Howitt, Marie McFarland, Elizabeth Garcia, Lynette M Sholl, Neal I Lindeman, Laura E MacConaill, Fei Dong, Michelle S Hirsch, Marisa R Nucci, Charles $M$ Quick, Christopher P Crum, W Glenn McCluggage, Jelena Mirkovic. Brigham and Women's Hospital, Boston, MA; Belfast Health and Social Care Trust, Belfast, Northern Ireland, United Kingdom; University of Arkansas for Medical Sciences, Little Rock, AR. Background: Mesonephric carcinoma (MCA), a malignant gynecologic tumor most commonly arising in the cervix and presumed to be derived from mesonephric remnants (MR), is characterized by recurrent $K R A S$ mutations. Adenocarcinomas of the uterine corpus and ovary characterized by morphologic and immunophenotypic similarities to MCA have been reported, without associated MR or cervical involvement. The pathogenesis of these tumors, which have been designated "Mesonephric-like adenocarcinomas (MLAS)" is not well understood, and it has been debated whether these represent MCAs that arise in the endometrium/ovary, or endometrioid adenocarcinomas that closely mimic MCA. The relationship at the molecular level between MLAs of the uterine corpus and ovary, and MCA is unknown. The aim of this study was to examine the molecular alterations in MLAs to identify driver mutations, potential therapeutically targetable mutations, and to determine their relationship to MCA using targeted next generation sequencing.

Design: 7 MLAs (4 ovary, 3 uterine corpus) with available formalin-fixed paraffin embedded tumor were contributed by 2 of the authors (WGM, CMQ). Genomic DNA was isolated and targeted next generation sequencing was performed to detect mutations, copy number variations and structural variants in exonic regions of 300 cancer genes and 113 selected intronic regions across 35 genes.

Results: All 7 tumors harbored canonical activating KRAS mutations (4 G12D, 3 G12V) PIK3CA mutations (all missense, previously reported somatic) were identified in 3 of 7 (43\%) cases. 2 of 7 cases ( $29 \%$ ) harbored ARIDIA mutations (inframe deletions). There were no alterations in ARIDIB, SMARCA4, PTEN, or TP53 in any of the tumors. 5 of $7(71 \%)$ tumors exhibited 1q gain, which was accompanied by $1 \mathrm{p}$ loss in two cases. In addition, 4 of 7 (57\%) tumors had chromosome 10 gain, which was accompanied by gain of chromosome 12 in 3 cases.

Conclusions: MLAs, similar to MCAs, are characterized by recurrent $K R A S$ mutations, gain of 1q, lack of PTEN mutations, and gain of chromosomes 10 and 12. PIK3CA mutations, which were not previously identified in MCA, were found in 3 of $7(43 \%)$ MLAs in our study. MLAs exhibit strikingly similar molecular aberrations to MCAs, suggesting that they exhibit mesonephric rather than endometrioid differentiation.

1164 The Pathologic Distinction of Primary Mucinous from Metastatic Tumors of the Ovary: A Re-Evaluation of Algorithms Based on Gross Features

Jingjing Hu, Andres A Roma, Vinita Parkash, Oluwole Fadare. UCSD, San Diego, CA; Yale, New Haven, CT.

Background: Algorithms that are based on tumoral gross features have been proferred as a useful way to distinguish primary mucinous from secondary (metastatic) tumors of the ovary, especially during intraoperative assessments. Using a relatively large dataset, we 
systematically re-evaluated a variety of gross parameters to determine the combination of features that most optimally distinguished primary from secondary tumors, and to identify the tumor types that are most frequently associated with exceptions

Design: Gross features were documented in 129 tumors involving the ovary, including 61 mucinous tumors classified as primary ( 16 carcinomas, 45 borderline tumors), and 68 classified as metastatic ( 21 colon; 28 appendix; 5 breast; 3 lung; 3 pancreas; 3 cervix; 1 bladder; 4 stomach). Gross features - tumor size, bilaterality, cut surface, and surface nodules - that were most predictive of metastatic status were determined. Receiver operating curve (ROC) analyses were used to select the optimal distinguishing size, which were assessed at each integer from $9 \mathrm{~cm}$ to $17 \mathrm{~cm}$

Results: Tumor size was found to be the most powerful predictor of metastatic status. ROC analysis showed that a size cut off of $<13 \mathrm{~cm}$ [for metastatic disease] yielded the maximal AUC of 0.8534 (sensitivity $80 \%$; specificity $80 \%$ ). Frequent exceptions to that size cut off included colon and the rare cases that originated from the cervix, stomach, and lung, in all of which $25-33 \%$ of metastatic tumors were $\geq 13 \mathrm{~cm}$. By contrast, appendix $(14 \%)$, pancreas $(0 \%)$ and breast $(0 \%)$ only rarely violated that $<13 \mathrm{~cm}$ cut off. Consistent with previously reported findings, metastatic tumors were more likely than primary tumors to be bilateral ( $46 \%$ vs $3 \%$ ), to display surface nodules ( $54 \%$ vs $0 \%$ ), be smaller $(7.5 \mathrm{~cm}$ vs $20.54 \mathrm{~cm})$ and were less likely to have been described as being purely cystic ( $33 \%$ vs $70 \%$ ), $p<0.05$ for all. Bilaterality and surface nodules were each highly specific for metastases, but neither was optimally sensitive. However, an algorithm whereby a tumor $\geq 13 \mathrm{~cm}$ is considered primary unless it displays surface nodules or bilaterality, and a tumor $<13 \mathrm{~cm}$ is considered metastatic unless it is unilateral, correctly classifies $94 \%(64 / 68)$ of the metastatic tumors and $98 \%(60 / 61)$ of the primary tumors

Conclusions: Although gross features are imperfect in distinguishing primary mucinous from metastatic ovarian tumors, the algorithm outlined above can provide useful diagnostic information during an intraoperative consultation, as it accurately classified $\geq 94 \%$ of the tumors in this study

\section{Metastatic Tumors Involving the Ovary: Pathologic Features} Associated with Patterns of Clinical Presentation in 68 Cases

Jingjing Hu, Andres A Roma, Vinita Parkash, Oluwole Fadare. UCSD, San Diego, CA; Yale University, New Haven, CT.

Background: Metastatic tumors that involve the ovary can pose a significant diagnostic challenge to the pathologist during intraoperative assessment, especially when the existence of the primary tumor is not clinically known. We hypothesized that in metastatic tumors that involve the ovary, gross tumoral features are associated with the various clinical presentation patterns, and test that hypothesis herein

Design: 68 consecutive cases of metastatic tumors involving the ovary constituted our study set. The clinical presentations were classified into 3 groups: Group 1 (ovarian tumor is the first clinical indication of the primary tumor), Group 2 (ovarian tumor is the first recurrence of a known primary tumor that was clinically presumed to be in remission), Group 3 (a patient with known cancer with widely metastatic disease, including ovarian involvement). These 3 groups were statistically compared regarding patient age and gross features (tumor size, bilaterality, nature of cut surface, surface nodules)

Results: The 68 cases, comprised of metastatic tumors from the colon (21), appendix (28), breast (5), lung (3), pancreas (3), cervix (3), bladder (1), and stomach (4), were classified into group $1(\mathrm{n}=14)$, group $2(\mathrm{n}=24)$ and group $3(\mathrm{n}=30)$ using the aforementioned definitions; these groups are compared below:

\begin{tabular}{|l|l|l|l|l|l|l|}
\hline Feature & $\begin{array}{l}\text { group } \\
{[\mathrm{G}] 1}\end{array}$ & $\begin{array}{l}\text { group } \\
{[\mathrm{G}] 2}\end{array}$ & $\begin{array}{l}\text { group } \\
{[\mathrm{G}] 3}\end{array}$ & $\begin{array}{l}\text { G1 vs G3 } \\
\text { (p value) }\end{array}$ & $\begin{array}{l}\text { G2 vs G3 } \\
\text { (p value) }\end{array}$ & $\begin{array}{l}\text { G1 vs G2 } \\
\text { (p value) }\end{array}$ \\
\hline Number of cases & 14 & 24 & 30 & NA & NA & NA \\
\hline Patient age (yrs) & 45 & 53 & 53 & 0.1 & 0.82 & 0.08 \\
\hline Mean size (cm) & 12.3 & 8.4 & 4.9 & 0.0001 & 0.006 & 0.041 \\
\hline $\begin{array}{l}\text { Surface nodules } \\
\text { present (\%) }\end{array}$ & 57 & 25 & 77 & 0.29 & 0.0003 & 0.81 \\
\hline Purely cystic (\%) & 50 & 25 & 27 & 0.18 & 1 & 0.2 \\
\hline Bilateral (\%) & 43 & 58 & 40 & 0.7 & 0.3 & 0.5 \\
\hline $\begin{array}{l}\text { Ovaries less than } 10 \\
\text { cm (\%) }\end{array}$ & $35(7 / 20)$ & $\begin{array}{l}61 \\
(20 / 33)\end{array}$ & $\begin{array}{l}95 \\
(40 / 42)\end{array}$ & $<0.0001$ & 0.0001 & 0.05 \\
\hline
\end{tabular}

Conclusions: Clinical presentation patterns showed significant gross pathologic correlates. Patients whose metastatic mass was the first presentation of their disease had significantly larger ovarian masses, and there was a trend towards a comparatively younger age for this group of patients. $65 \%$ of the group 1 ovaries were $\geq 10 \mathrm{~cm}$, as compared with $39 \%$ and $15 \%$ of the groups 2 and 3 ovaries. All groups showed bilaterality and surface nodules at comparable frequencies. The tendency for group 1 patients to present with comparatively larger masses is a potential source for misclassification during intraoperative assessments if the primary versus secondary tumor determination is overly dependent on size-based algorithms

1166 Liquid Based Cytology: Do Ancillary Techniques Enhance Detection of Epithelial Abnormalities?

Nuzhat Husain, Sridhar Mishra, Namrata P Awasthi, Yashodhara Pradeep. Dr Ram Manohar Lohia Institute of Medical Sciences, Lucknow, UP, India; King George's Medical University, Lucknow, UP, India.

Background: Cervical cancer represents a second place in global incidence with 500000 new cases each year, to which India contributes 100,000. Liquid Based Cytology (LBC) provides the use of ancillary techniques in addition to a good morphology and detection of cytologic abnormalities. The current study was designed to assess the diagnostics of P16INK4a immunoexpression, P16 promoter hypermethylation, Human Papilloma Virus (HPV) \& DNA ploidy in LBC samples with cervical precancer and cancer.
Design: A series of LBC samples categorised by Bethesda system with epithelial abnormalities inlcuding 22 Atypical Squamous Cells of Undetermined Significance (ASC-US), 21 Low Grade Squamous Intraepithelial Lesion (LSIL), 41 High Grade Squamous Intraepithelial Lesion (HSIL), 54 Squamous Cell Carcinoma (SCC) and 26 controls with normal cytology were included. Ancillary techniques evaluated included P16INK4a gene immunoexpression, P16 promoter methylation by methylation-specific PCR, DNA ploidy in flowcytometry (FCM) \& HPV was detected by conventional PCR with PGMY09/PGMY11 primers.

Results: The sensitivity and specificity of each ancillary techniques in Bethesda categories is summarised in table 1 . The mean of Diploid cell cycle parameter values in control, LSIL, HSIL and SCC was 94.7 $\pm 3.44,92.17 \pm 4.36,67.85 \pm 18.89$ and 63.9 \pm 17.69 respectively and the mean of the diploid S values in control, LSIL, HSIL and SCC was $4.63 \pm 0.47,7.42 \pm 4.13,32.14 \pm 9.05$ and $35.6 \pm 18.7$.

\begin{tabular}{|l|l|l|l|l|l|l|}
\hline \multicolumn{7}{|c|}{ Table 1: Sensitivity and Specificity of Ancillary Techniques in LBC } \\
\hline $\begin{array}{c}\text { Epithelial } \\
\text { Abnormality }\end{array}$ & $\begin{array}{c}\text { P16 } \\
\text { Expression }\end{array}$ & $\begin{array}{c}\text { P16 } \\
\text { methylation }\end{array}$ & HPV & $\begin{array}{c}\text { Diploid G1 } \\
\text { Value }\end{array}$ & $\begin{array}{c}\text { P16 } \\
\text { Diploid S } \\
\text { Value }\end{array}$ & $\begin{array}{c}\text { Expression } \\
+ \text { P16 } \\
\text { Methylation } \\
+ \text { HPV }\end{array}$ \\
\hline ASC-US & $25.05 / 100$ & $36.36 / 100$ & $\begin{array}{c}45.45 / \\
96.15\end{array}$ & & & $62.93 / 96.3$ \\
\hline LSIL & $36.39 / 100$ & $76.19 / 100$ & $\begin{array}{c}76.19 / \\
96.15\end{array}$ & $50.0 / 87.10$ & $\begin{array}{l}50.0 / \\
87.10\end{array}$ & $50.0 / 96.3$ \\
\hline HSIL & $67.65 / 100$ & $98.68 / 100$ & $\begin{array}{c}87.80 / \\
96.15\end{array}$ & $65.0 / 100$ & $70.0 / 100$ & $71.43 / 96.3$ \\
\hline SCC & $87.37 / 100$ & $92.59 / 100$ & $\begin{array}{l}92.59 / \\
96.15\end{array}$ & $\begin{array}{l}78.57 / \\
96.77\end{array}$ & $\begin{array}{l}71.43 / \\
100\end{array}$ & $88.10 / 96.30$ \\
\hline Numbers denote sensitivity/ specificity
\end{tabular}

Conclusions: P16 gene promoter methylation analysis seems to be more sensitive and specific in detection of pre-neoplastic lesions. Its combination with P16 and HPV would play a pivotal role. Diploid G1 and diploid S phase analysis do not appear to increase the overall sensitivity \& specificity of detection however the study brings front a progressive change in parameters with progression from LSIL to HSIL \& SCC.

\section{Loss of 5-Hydroxymethylcytosine $(5 \mathrm{hmC})$ Protein Expression} in Endometrioid Type Endometrial Carcinoma

Hiba Ibrahim, Karen Dresser, Kristine Cornejo. University of Massachusetts Medical School, Worcester, MA.

Background: Epigenetic alterations such as DNA methylation are important in cancer development. Recently, the epigenetic mark 5-hydroxymethylcytosine (5hmC) has been shown to be reduced in a variety of cancers and involved in the process of malignant transformation. To date, no studies have examined the expression of $5 \mathrm{hmC}$ in endometrium and endometrial carcinoma. The aim of our study was to investigate $5 \mathrm{hmC}$ protein expression in proliferative endometrium (PE) and endometrioid type endometrial carcinoma (ETEC).

Design: We examined the expression of $5 \mathrm{hmC}$ by immunohistochemistry in a total of 60 cases comprised of $29 \mathrm{PE}$ and 31 ETEC. The percentage of nuclear staining was estimated for each case. Endometrial stroma was used as a positive control for the $5 \mathrm{hmC}$ staining.

Results: Among the 29 PE cases, 28 (97\%) had strong and diffuse staining with minimal to no loss of $5 \mathrm{hmC}$ expression ( $<25 \%$ of the cells) (Table 1). A loss of $5 \mathrm{hmC}$ staining was observed in $>25 \%$ of the cells in 25 of 31 (81\%) ETEC cases, of which $16(52 \%)$ of these cases displayed a loss in $>50 \%$ of the tumor cells. The loss of $5 \mathrm{hmC}$ expression was significantly increased in ETEC cases in comparison to the PE cases $(\mathrm{p}<0.0001)$ by Fishers exact test.

Conclusions: Our study demonstrates $5 \mathrm{hmC}$ expression is significantly reduced in ETEC compared with PE. These findings suggest that the loss of $5 \mathrm{hmC}$ is an epigenetic event in ETEC and may play a role in its development and progression.

Table 1 . Summary of cases with $5 \mathrm{hmC}$ expression.

\begin{tabular}{|l|l|l|l|}
\hline Diagnosis & $0-25 \%$ Loss & $26-50 \%$ Loss & $>50 \%$ Loss \\
\hline PE $(\mathrm{n}=29)$ & $28(97 \%)$ & $0(0 \%)$ & $1(3 \%)$ \\
\hline ETEC $(\mathrm{n}=31)$ & $6(30 \%)$ & $9(29 \%)$ & $16(52 \%)$ \\
\hline
\end{tabular}

\section{Cervical Cancer: A Retrospective Analysis of the Utility of HPV} and Pap Testing for Women 60 Years and Older

Lauren Isbell, Riyam Zreik, Parsa Hodjat, Kimberly Walker, Teresa Ponder, Arundhati Rao. Baylor Scott and White, Temple, TX.

Background: In developed countries, the incidence and mortality rate of cervical cancer has decreased in the last several decades, with the implementation of routine Papanicolaou (Pap) and Human Papilloma virus (HPV) testing recommended by current guidelines [1]. The latest guidelines recommend that women stop cervical cancer screening after age 64 years old if they meet certain criteria, including no history of moderate or severe dysplasia or cancer and no recent abnormal Pap results, though the incidence of cervical cancer or related death rates has not significantly declined over the last decade for women ages 60 years old and above [2]. Interestingly, there are no guidelines available in regards to cervical cancer screening for women over 64 years of age. This study is meant to determine if there is utility in Pap/HPV testing for women over the age of 64 years old.

Design: Results including HPV tests, Pap results and biopsy results were obtained from 3,866 female patients 60 years and older who had HPV and Pap tests at Scott and White Temple Hospital between 01/2012 and 07/2016, with emphasis on cases with a minimum of 3 year follow up. The frequency of abnormal test results in HPV positive patients and overall patients (table 1) were analyzed. 
Results: Among the 3,866 women 60 years and older who had HPV testing, 247 (6.4\%) of them had positive high risk HPV results. Of those women, $127(5.7 \%)$ women were between 60 and 64 years old and $120(7.4 \%)$ women were over 64 years old. $2.3 \%$ of women between 60 and 64 years old with abnormal results had follow-up biopsies with cervical dysplasia or malignancy while $2.9 \%$ of women over 64 years old were diagnosed with dysplasia or malignancy. In the group of women with positive HPY results, $66(52.0 \%)$ women between 60 and 64 years old had abnormal Pap results and $51(42.5 \%)$ women over 64 years old had abnormal Pap results. The majority of women with cervical biopsy proven abnormalities in the age group above 64 years old were newly diagnosed compared to the younger group.

\begin{tabular}{|c|c|c|c|c|c|}
\hline & \multicolumn{3}{|c|}{ HPV +} & \multicolumn{2}{c|}{ Overall } \\
\hline Age & Total & Abn. Pap & CIN/Cancer & Abn. Pap & CIN/Cancer \\
\hline $60-64$ & $127(5.7 \%)$ & $66(52.0 \%)$ & $31(24.4 \%)$ & $147(6.6 \%)$ & $51(2.3 \%)$ \\
\hline$>64$ & $120(7.4 \%)$ & $51(42.5 \%)$ & $31(25.8 \%)$ & $123(5.5 \%)$ & $47(2.9 \%)$ \\
\hline
\end{tabular}

Conclusions: Given the similar frequency of cervical dysplasia or malignancy among women 60-64 years old and over 64 years old, it might be beneficial to consider continued cervical cancer screening for women over 64 years old

$1169 \quad$ Accentuated p53 Staining in Usual Type Vulvar Dysplasia, a Potential Diagnostic Pitfall

Matthew Jeffreys, Susanne Jeffus, Charles M Quick. UAMS, Little Rock, AR.

Background: Evaluation of vulvar dysplasia may be difficult due to overlapping histologic features in both usual (uVIN) and differentiated vulvar intraepithelial neoplasia (dVIN). The diagnosis of dVIN is particularly vexing, and has poor interobserver variability. P53 has been described as a helpful adjunct in some cases; however, intricacies in its evaluation abound. This study details an accentuated wild-type pattern that may lead to the erroneous diagnosis of dVIN.

Design: 42 consecutive cases of vulvar dysplasia (23 keratinizing / suggestive of dVIN 19 consistent with $\mathrm{UVIN}$ ), and 5 normal cases (total $\mathrm{n}=47$ ) were reviewed and stained for p16 \& p53. P16 was scored as positive (block positive staining) or negative (patchy no staining). P53 was interpreted as positive (intense, confluent, basal staining), wild-type (WT) (scattered weakly positive cells), and accentuated (increased intensity, frequency $5-50 \%$, throughout the epithelium, sparing basal cells).

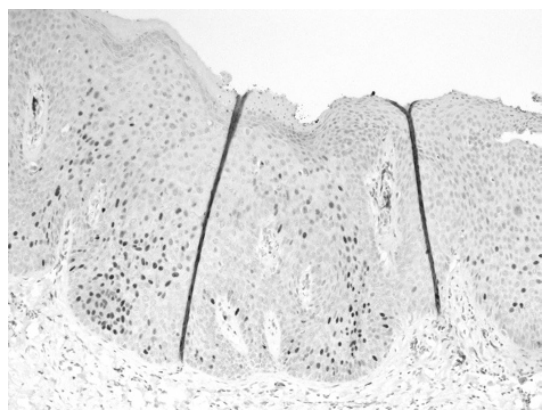

Histologic features were recorded (basal atypia, superficial keratinization, koilocytes, basaloid architecture). Follow up pathology was recorded if available.

Results: Accentuated WT p53 staining was seen in 22 cases (12/23 suggestive of dVIN $(52 \%), 10 / 19(52 \%)$ consistent with uVIN). P16 was positive in all 22 of these cases. Positive p53 was seen in 4 of $23(17 \%)$ cases suggestive of dVIN and one histologically normal case; p16 was negative in these 5 cases. P16 was positive in 35 of 42 cases $(83 \%)$, all of which were either p53 WT $(13,37 \%)$ or accentuated $(22,63 \%)$. Using strict definitions for $\mathrm{p} 53$ and $\mathrm{p} 16$ positivity, no $\mathrm{p} 53$ positive cases were also positive for $\mathrm{p} 16$, contrary to previous studies, which describe such staining. All cases of dVIN displayed prominent basal atypia and p53 positivity; 3 of 4 progressed to carcinoma. Only 1 of 35 patients with p16 positive uVIN progressed to carcinoma.

Conclusions: Differentiated VIN is difficult to diagnose due to potential histologic overlap with uVIN, especially when keratinization is present. Accentuated WT p53 staining may lead to misdiagnosis as dVIN, especially in small samples. P16 is a reliable marker, and tandem $\mathrm{p} 16 / \mathrm{p} 53$ staining should be performed with strict adherence to patterns considered positive, as patients with uVIN that are p16 positive have significantly less risk of progression.

1170 Prevalence and Genotype Distribution of High Risk HPV Infection and Cervical Cytology of Women with CIN 2/3 in Southwest China Wei Jiang, Christopher C Griffith, Kaixuan Yang, Chengquan Zhao. West China Second University Hospital, Sichuan University, Chengdu, Sichuan, China; Emory University, Atlanta, GA; Magee-Women's Hospital of UPMC, Pittsburgh, Pittsburgh, PA.

Background: Data regarding HPV genotype distribution for women with CIN2/3 are limited and vary by country and region.

Design: CIN2/3 cases with prior HPV testing and cervical cytology data in the preceding 6 months were collected at a large university women's hospital in China.HPV detection was performed using two kinds of genotyping Assays. Pap cytology was performed with ThinPrep and SurePath methods.

Results: 2771 CIN 2/3 cases with prior HPV testing were included in this study. The overall HPV prevalence was $91.7 \%$ and the hrHPV prevalence was $90.1 \%$. Among 2499 cases with positive hrHPV testing, the most common types were HPV-16 (53.3\%), $58(21.4 \%), 52(11 \%), 33(10.6 \%)$ and $18(5.1 \%) .85 .8 \%$ of cases were positive for only hrHPV types and $69.3 \%$ were positive for a single hrHPV type. 1094 cases had both HPV and Pap cytology, only $4.7 \%$ of whom were normal cytology, significantly lower than $10.1 \%$ negative hrHPV rate. Only 4 cases $(0.4 \%)$ are negative by both HPV testing and Pap cytology. The negative hrHPV rates between the two HPV testing methods and the Pap cytology negative rates between the two Pap methods showed no significant differences.

\begin{tabular}{|l|l|l|l|l|l|l|}
\hline \multirow{2}{*}{ Type } & \multicolumn{2}{l|}{$\begin{array}{l}\text { YanengBIO method } \\
(1338 \text { cases })\end{array}$} & \multicolumn{2}{l|}{$\begin{array}{l}\text { TELLGEN method (1433 } \\
\text { cases) }\end{array}$} & \multicolumn{2}{l|}{ Total (2771 cases) } \\
\cline { 2 - 7 } & $\begin{array}{l}\text { Positive } \\
\text { No }\end{array}$ & Prevalence & $\begin{array}{l}\text { Positive } \\
\text { No }\end{array}$ & Prevalence & $\begin{array}{l}\text { Positive } \\
\text { No }\end{array}$ & Prevalence \\
\hline Single HR & 927 & $69.3 \%$ & 994 & $69.4 \%$ & 1921 & $69.3 \%$ \\
\hline Multiple HR & 223 & $16.7 \%$ & 234 & $16.3 \%$ & 457 & $16.5 \%$ \\
\hline Mix HR+LR & 60 & $4.5 \%$ & 61 & $4.3 \%$ & 121 & $4.4 \%$ \\
\hline Summary & 1210 & $90.4 \%$ & 1289 & $90.0 \%$ & 2499 & $90.1 \%$ \\
\hline
\end{tabular}

\begin{tabular}{|l|l|l|l|l|l|l|l|}
\hline & Total No & HSIL & LSIL & ASC-US & ASC-H & AGC & Negative \\
\hline SurePath & 149 & $\begin{array}{l}54 \\
(36.2)\end{array}$ & $\begin{array}{l}44 \\
(29.5)\end{array}$ & $\begin{array}{l}24 \\
(16.1)\end{array}$ & $\begin{array}{l}15 \\
(10.1)\end{array}$ & $\begin{array}{l}3 \\
(2.0)\end{array}$ & $\begin{array}{l}9 \\
(6.0)\end{array}$ \\
\hline ThinPrep & 945 & $\begin{array}{l}412 \\
(43.6)\end{array}$ & $\begin{array}{l}259 \\
(27.4)\end{array}$ & $\begin{array}{l}122 \\
(12.9)\end{array}$ & $\begin{array}{l}99 \\
(10.5)\end{array}$ & $\begin{array}{l}15 \\
(1.6)\end{array}$ & $\begin{array}{l}38 \\
(4.0)\end{array}$ \\
\hline Summary & 1094 & $\begin{array}{l}466 \\
(42.6)\end{array}$ & $\begin{array}{l}303 \\
(27.7)\end{array}$ & $\begin{array}{l}146 \\
(13.4)\end{array}$ & $\begin{array}{l}114 \\
(10.4)\end{array}$ & $\begin{array}{l}18 \\
(1.6)\end{array}$ & $\begin{array}{l}47 \\
(4.3)\end{array}$ \\
\hline
\end{tabular}

Conclusions: This is the largest case study of prior HPV genotyping and cytology in women with histologic CIN2/3. The prevalences of hrHPV genotypes for these women differed from that of other studies and this could be important for continued HPV vaccine development. In addition, about $10 \%$ of CIN $2 / 3$ cases had a recent negative hrHPV testing, exposing the limitation for the potential use of HPV only testing as a primary screening strategy.

1171 Endometrial Biopsy of Dedifferentiated and Undifferentiated Endometrial Carcinoma, Emphasizing Useful Diagnostic Features in Under-Sampled Tumors That Otherwise Appear as Low Grade Endometrioid Adenocarcinoma

Amy S Joehlin-Price, Joseph Rabban. UCSF, San Francisco, CA.

Background: Dedifferentiated endometrial carcinoma (DDEC) is an uncommon aggressive tumor composed of undifferentiated endometrial carcinoma (UEC) and low grade endometrioid adenocarcinoma (EMCA). Since surgical management as a high grade tumor is warranted, accurate diagnosis on endometrial biopsy is needed. We hypothesize that sampling issues may result in some biopsies of DDEC showing only the EMCA component, leading to surgical planning for a low grade tumor. Morphologic features in under-sampled biopsies of DDEC have not been well studied.

Design: Endometrial biopsy specimens from 23 hysterectomy-confirmed DDEC (18) or UEC (5) were retrospectively classified using 2014 WHO criteria as EMCA, UEC, DDEC, or descriptively as EMCA with a component of solid pattern tumor suspicious for DDEC. EMA and keratin immunostaining was used to confirm morphologic suspicion for UEC versus higher grade EMCA in challenging cases.

Results: Only $12 / 23(52 \%)$ biopsies contained definitive features of DDEC (10) or pure UEC (2). In these 12, morphologic features included discohesion (12), geographic necrosis (9), monomorphic nuclear atypia (10), rhabdoid cells (8), corded growth (9), and myxoid stroma (8). The UEC component comprised $30-95 \%$ of DDEC cases. The remaining 11/23 (48\%) cases lacked classic features of DDEC/UEC and consisted of pure atypical hyperplasia (AH) (2), grade 1 or 2 EMCA with (3) or without (4) a minor ( $5 \%$ to $20 \%$ ) component of solid pattern tumor suspicious for DDEC, and high grade adenocarcinoma, not further classifiable (1). An additional biopsy contained scant malignant cells (1). Rare diagnostic clues in the 3 cases with a suspicious minor solid pattern tumor included focal discohesion (2), monomorphic nuclear atypia (2), rhabdoid cells (2), and myxoid stroma (1). Among the 6 pure low grade EMCA/AH biopsies, $3 / 6$ had pools of necrotic debris, occupying $1 \%$ to $95 \%$ of the biopsy, and $2 / 6$ had intraluminal necrotic debris in the tumor itself. None had myxoid stroma. Tumor infiltrating lymphocytes were present in $1 / 6$.

Conclusions: Sampling artifact of DDEC may result in under-diagnosis by endometrial biopsy in nearly half of cases. Biopsies containing lower grade EMCA with abundant necrosis or with solid components exhibiting discohesive tumor cells may represent under-sampled DDEC. Immunohistochemical work up should be considered and, at a minimum, the possibility of DDEC should be discussed in the report in order to prompt further clinical evaluation and appropriate surgical planning.

\section{E-Cadherin/CD34 Dual Immunohistochemical Stain in Search} for Placental Focal Fetal Vascular Malperfusion

Sheryl L Johnson, Jerzy Stanek. Cincinnati Children's Hospital Medical Center, Cincinnati, $\mathrm{OH}$.

Background: Fetal thrombotic vasculopathy (FTV) typically features clusters of avascular chorionic villi on histological examination but its early stages may not be seen on hematoxylin-eosin (H\&E) stained placental sections, and thus overlooked. E-cadherin/CD34 immunostain (EC-CD34) used to highlight villous cytotrophoblasts, vascularity, and vasculosyncytial membranes in the diagnosis of chronic placental hypoxia has not previously been used to detect FTV.

Design: Representative sections of 38 placentas from high-risk pregnancies, mostly perinatal mortality, were stained for EC-CD34. 20 clinical and 40 placental phenotypes were statistically (Chi-square or ANOVA) compared between Group 1 with FTV on $\mathrm{H} \& \mathrm{E}$ and/or EC-CD34 and Group 2 without FTV. 
Results: Group 1 contained 22 cases: 12 placentas featuring clusters of avascular chorionic villi on routine $\mathrm{H} \& \mathrm{E}$ slides, including 6 with also abnormal EC-CD34 pattern, and 10 placentas with abnormal EC-CD34 pattern only (figure 1) with (insert) or without endothelial disruption in areas free of FTV on H\&E stained slides.

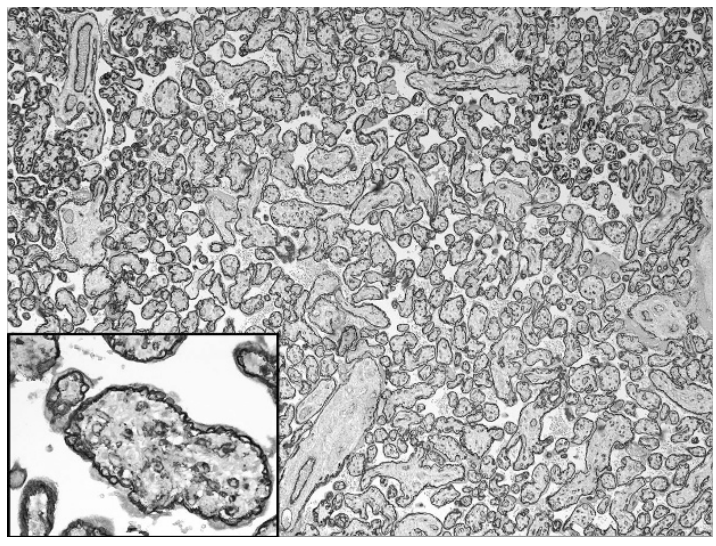

Group 2 contained 16 cases without FTV. There were no statistically significant differences in clinical phenotypes between Groups 1 and 2. Of placental phenotypes, inflammatory lesions (acute chorioamnionitis, chronic villitis, plasma cell deciduitis) were seen in 18 vs $56 \%$ of cases $(\mathrm{p}=0.01$ ), excessive amount of chorionic disc extravillous trophoblasts in 50 vs $19 \%(\mathrm{p}=0.05)$, and ectasia of chorionic or stem vessels in 50 vs $19 \%(\mathrm{p}=0.05)$, thus disclosing the likely umbilical cord compromise and inflammation dominating Group 1 vs Group 2, respectively.

Conclusions: This retrospective study shows that the abnormal EC-CD34 pattern is able to highlight early stages of FTV before clusters of avascular chorionic villi become manifest on H\&E stained sections. Furthermore, even in the latter situation, the abnormal EC-CD34 pattern can disclose the ongoing thrombotic process, thus upgrading the FTV. We believe that the EC-CD34 immunostaining has a potential to increase the sensitivity of placental examination in retrospectively diagnosing the umbilical cord compromise which is a common cause of fetal/neonatal morbidity/mortality, frequently eluding the placental confirmation otherwise.

\section{Amplification of the NSD3-CHD8-BRD4 Axis in Pelvic High-} Grade Serous Carcinomas of Tubo-Ovarian and Endometrial Origin

Derek Jones, Douglas Lin. Beth Israel Deaconess Medical Center, Boston, MA.

Background: Pelvic high-grade serous carcinoma (HGSC) is an aggressive and devastating neoplasm, however identification of novel therapeutics has been hampered by paucity of actionable point mutations in target genes. In recent years, chromatin regulators, including BRD4, have become attractive targets for cancer therapy. NSD3 is a methyltransferase that couples BRD4 to the chromatin remodeling factor CHD8 to activate transcription of oncogenic drivers such as CMYC.

Design: We analyzed The Cancer Genome Atlas (TCGA) ovarian HGSC and endometrial carcinoma cohorts to test the association of NSD3, CHD 8 and BRD4 genomic alterations with patient survival and clinicopathological characteristics of pelvic HGSC

Results: Here we demonstrate that somatic amplification of NSD3-CHD8-BRD4 pathway (seen in $18 \%$ of cases, 88 of 489 , of the ovarian carcinoma TCGA cohort is significantly associated with worse overall and progression-free survival relative to non-amplified cases in ovarian HGSC. In addition, analysis of the endometrial carcinoma TCGA cohort also revealed amplification of NSD3, CHD8 and BRD4, in $9 \%$ ( 21 of 232) of overall endometrial cancer cases, which was associated with worse overall survival. In the endometrial cancer TCGA cohort, NSD3, CHD 8 and BRD4 amplification occurred specifically in the serous carcinoma (seen in $25 \%, 13$ of 53 ) and "serous-like" copy number high endometrial carcinoma (seen in 33\%, 20 of 60 cases) subgroups, compared to the POLE $(0 \%, 0$ of 17$)$, MSI high $(0 \%, 0$ of 65$)$ or low copy number $(1 \%, 1$ of 90$)$ subgroups.

Conclusions: These findings support the notion that amplification of the NSD3-BRD4$C D H 8$ axis are frequent events in pelvic high grade serous carcinomas of both ovarian and endometrial origin and that trials with the newly developed BRD4, BET small molecule inhibitors are warranted in a subset of patients with pelvic HGSC.

1174 Expression of PD-L1 and GHRH-R in Uterine Carcinosarcoma: Potential Markers for Targeted Therapy

Mirka W Jones, Dinesh Pradhan, Agnieszka Onisko, Rohit Bhargava, David J Dabbs. University of Pittsburgh Medical Center, Pittsburgh, PA.

Background: Uterine carcinosarcomas (UCS) are rare malignant neoplasms with very aggressive behavior. They account for $15 \%$ of all deaths due to uterine corpus malignancy. Despite therapeutic advances, the prognosis of patients with UCS remains poor, with 5-year survival of $33-39 \%$. Recently, it was discovered that the use of immunotherapeutic drugs targeting programmed cell death protein 1 (PD1)/ programmed death ligand-1 (PD-L1) axis is associated with improved survival in several types of cancer. So far, the expression of PD-L1 in UCS has not been studied. Another potential therapeutic target is growth hormone releasing hormone (GHRH), found in many malignant tumors. The antagonists against GHRH receptors (GHRH-R) have shown great efficacy in the experimental models. The aim of this study is to evaluate the expression and therefore the potential therapeutic value of both PD-L1 and GHRH-R in UCS.
Design: A computer-based search was carried out on our pathology database to retrieve the UCSs diagnosed between 2007 and 2012. The clinicopathologic, treatment and outcome information was obtained from the medical records. Representative sections were immunostained with PD-L1 and GHRH-R. The results of PD-L1 immunostaining was scored in the tumor and in the tumor infiltrating immune cells (TII) and the GHRH immunostaining was graded semi-quantitatively (H score) in the epithelial and stromal component by two pathologists. UCS with PD-L1 staining in $\geq 5 \%$ of the tumor cells were designated PD-L1 positive and $\mathrm{H}$ score $\geq 150$ for GHRH-R was designated as positive. Results: Forty three UCSs were selected. The median age of the patients was 67 years (46-83 years). The average size of the tumor was $7.6 \mathrm{~cm}(1.8-16 \mathrm{~cm})$. Seventeen tumors $(39.5 \%)$ were stage I, $4(9.3 \%)$ were stage II, $15(34.9 \%)$ were stage III and $7(16.3 \%)$ were stage IV. PD-L1 was strongly positive in $24 \mathrm{UCS}$ (strong staining in 5-70\% of cells) and 18 UCS revealed strongly positive TII ( $5-50 \%$ of cells). GHRH-R was positive in the epithelial component of 33 UCS (H-score $\geq 150)$ and in the mesenchymal component of 10 UCS. There was a statistically significant difference in expression of GHRH-R between the epithelial and stromal component.

Conclusions: The majority of UCS showed very strong and diffuse expression of PDL1 $(56 \%)$ and GHRH-R $(77 \%)$. Although additional studies are needed, these findings suggest a possible rationale for therapeutic targeting of PD-L1 pathway and GHRH receptors in the treatment of UCS.

1175 Single Cell Reactivity Is a Distinct Pattern of DNA Mismatch Repair Protein Expression in Endometrial Carcinoma

D Anand Rajan Kanagasabapathy, Ian Hagemann. Carver College of Medicine, University of Iowa Healthcare, Iowa City, IA; Washington University School of Medicine, St Louis, MO

Background: Endometrial carcinoma (EMC) is one manifestation of Lynch syndrome, which is driven by DNA mismatch repair (MMR) protein defects. These result in abnormal expression of one or more MMR proteins MSH2, MSH6, MLH1 and PMS2. Immunohistochemistry can be used to identify this phenotype in a tissue context. We recently identified a pattern of MMR protein reactivity that has not been discussed in the literature. We sought to characterize the pattern in a consecutive set of cases and measure its impact on interobserver agreement of MMR IHC assessment in a group of subspecialized gynecologic pathologists.

Design: We reviewed a series of endometrial carcinomas that were clinically reported as having normal $(\mathrm{n}=20)$ or abnormal $(\mathrm{n}=25)$ MMR protein expression. Immunostains (MLH1, PMS2, MSH2, MSH6) and H\&E sections from each case were reviewed by 5 pathologists in an independent, blinded manner. Ratings were recorded as retained vs. lost. Single cell reactivity pattern (SCR) was recorded when present, and was defined as occasional single tumor nuclei showing definite positivity in a backdrop of predominantly negative tumor.

Results: Agreement between individual observers and the clinically reported overall MMR status varied between $53.3 \%$ and $93.3 \%$ with a mean of $77.8 \%$. Significant variability was observed for MLH1 (64.4-88.9\%) and PMS2 (62.2-79.6\%) compared to MSH2 (95.6-100\%) and MSH6 (91.1-97.8\%). Full consensus (agreement between all observers) was noted in roughly half the cases for MLH1 (55\%) and PMS2 (48\%) whereas it was $>93 \%$ for MSH2 and MSH6. SCR was identified by at least one rater in $13 / 45(29 \%)$ of cases. Nearly all SCR was seen in MLH1 and PMS2, excepting one MSH2. Out of 13 cases, 10 were reported clinically as having loss of MMR expression. There was a statistically significant correlation between SCR and lack of consensus: of 17 cases with consensus, only one exhibited SCR, and of 13 SCR cases only one was agreed upon by all pathologists $(\chi 2=6 ; \mathrm{p}=0.01)$.

Conclusions: We hereby describe a novel pattern of MMR protein reactivity in EMC, seen mainly in MHL1 and PMS2. The pattern is (a) frequently reported as MMR loss, although some expression is clearly present, and (b) a significant source of interobserver disagreement between pathologists in MMR IHC reporting. Correlation with microsatellite instability and MLH1 promoter hypermethylation status is necessary to establish its biologic and clinical significance.

\section{Tubal and Ovarian Epithelial Cancer in Women with Germline} Mutations Other Than the BRCA and Lynch Syndrome Genes

Yuna Kang, Lee-may Chen, Julie Mak, Joseph Rabban. UCSF, San Francisco, CA. Background: Hereditary tubal / ovarian epithelial carcinoma is largely attributed to germline mutation of BRCA1 or BRCA2 but the advent of multi-gene panel testing has shown that a small subset involves other functionally-related genes. This study reports tubal / ovarian malignancies from a single institutional genetic counseling registry of women with a germline mutation other than BRCA1, BRCA2 or mismatch repair genes, who underwent salpingo-oophorectomy as part of cancer risk-reduction, gynecologic cancer treatment, or treatment of a non-gynecologic cancer.

Design: Patients were identified from our institution's genetic counseling registry based on presence of a positive test for germline mutation in any gene. Variants of unknown significance were excluded. This population was then cross-referenced with our surgical pathology registry to identify the subset of women who had undergone salpingo-oophorectomy for any indication at our institution. Clinical and pathologic findings were correlated in each case.

Results: Among 1,695 women with a mutation in any gene, $351(20.7 \%)$ underwent surgeries that included salpingectomy and/or oophorectomy. $331 / 351$ (94\%) had mutation in BRCA1, BRCA2 or a mismatch repair gene and were excluded from study. The remaining 20 had mutation of BRIP1 (3), PTEN (3), PALB2 (3), MUTYH monoallelic mutation (3), RAD51D (2), FH (2), TP53 (1), ATM (2; 1 of whom also had likely CHEK2 mutation), CHEK2 $(\mathrm{n}=1)$. Surgical indication was risk reduction in 11 women, 1 of whom (BRIP1) had serous tubal intraepithelial carcinoma (STIC). Pelvic mass was the indication for 5 women: 2 had pelvic high-grade serous carcinoma (HGSC) (MUTYH, 1; BRIP1, 1); 1 had metastatic colon cancer (MUTYH); and 1 
had high-grade sarcoma of the paravesical soft tissue (PTEN). Ovarian suppression for breast cancer was the indication for 3 women, 1 of whom (RAD51D) had occult metastatic breast cancer.

Conclusions: STIC and pelvic HGSC may occur in women with germline mutation of genes other than BRCA1, BRCA2 and mismatch repair genes, lending further support to the strategy of multi-gene panel testing to assess hereditary gynecologic cancer risk. Whether the morphology, prognosis and pathogenesis of these cancers differs from that in BRCA-related, Lynch syndrome-related or sporadic cancers will require multi-institutional collaboration of larger case numbers.

\section{$1177 \quad--H P V-I n d e p e n d e n t$ Vulvar Squamous Cell Carcinoma Has a} Worse Prognosis Than HPV-Associated Disease: A Retrospective Cohort Study

Anthony N Karnezis, Samuel Leung, Angela S Cheng, Dianne Miller, Aline Talhouk, C Blake Gilks, Jessica N McAlpine. University of British Columbia, Vancouver, BC, Canada.

Background: Vulvar squamous cell carcinoma (VSCC) can be subdivided based on HPV status into two molecularly distinct entities. Studies on the prognostic significance of HPV status in VSCC are discordant.

Design: To understand the significance of HPV status in the clinical behaviour of VSCC, we performed a retrospective analysis of overall survival (OS), disease-specific survival (DSS), and progression-free survival (PFS) in 217 patients with VSCC. Cases were extracted from a period encompassing an era of more aggressive en bloc radical dissections (1985-1995) and more localized radical surgery through separate vulvar and groin excisions (1996-2005). HPV status was determined by p16 immunohistochemistry. Results: HPV status could be determined in 197 tumours; 118 HPV-independent, 79 HPV-associated. Patients with HPV-associated tumours were younger (median 59 vs. 72 years for HPV-independent tumours, $\mathrm{p}<0.0001$ ) and more likely to have prior abnormal cervical cytology ( $41.1 \%$ vs. $5.6 \%$ for HPV-independent tumours, $\mathrm{p}<0.0001)$. HPV-associated and HPV-independent tumours showed similar stage distribution. In univariable analysis, patients with HPV-associated tumours had superior PFS (HR $0.37,95 \%$ CI $0.18-0.70$ ), DSS (HR $0.19,95 \%$ CI $0.08-0.41$ ) and OS (HR $0.35,95 \%$ CI 0.21-0.59). This was driven by worse outcomes (PFS, DSS and OS) for patients with HPV-independent tumours compared with those with HPV-associate tumours who underwent surgery after 1995 . After adjusting for age and stage in multivariable analysis, patients with HPV-associated tumours showed superior PFS (HR $0.25,95 \%$ CI 0.07-0.77) and DSS (HR 0.21,95\% CI 0.04-0.78).

Conclusions: VSCC can be stratified into two prognostically different diseases based on p16 immunostaining. HPV status was only associated with prognosis in the cohort that underwent surgery after 1995, suggesting more conservative surgery may have led to worse outcomes for patients with HPV-independent tumours. This highlights a potential role for molecular subtyping of VSCC in planning treatment and surveillance.

1178 mRNA-Based HPV Test Provides with More Accurate Genotyping Results for HPV16/18/45 Than DNA-Based HPV Test in Cervical Cancer Screening

Jana Kaspirkova, Jana Cimická, Barbora Gomolčáková, Ondrej Ondič, Michal Michal. Biopticka Laborator, Plzen, Czech Republic; Sikluv Ustav Patologie, Plzen, Czech Republic.

Background: Uterine cervical carcinoma $(\mathrm{CaCx})$ is the most common HPV-related neoplasm. Because of gradual character of HPV-induced cervical carcinogenesis, screening for early stages of disease and their surgical treatment could prevent vast majority of fatal cases. Traditional morphological methods of $\mathrm{CaCx}$ screening have been recently enriched by molecular genetic HPV detection which resulted in better sensitivity for high-grade cervical disease and prolonging of screening intervals. Partial genotyping of HPV infection represents important added value of HPV test which includes different risk-based management of HPV positive patients or better control of recurrent disease. Only a small number of HPV tests are recommended for $\mathrm{CaCx}$ screening purposes. It has been shown that mRNA-based HPV tests produce less false positive results because of substantial decrease of cross-reactivity with non-oncogenic HPV types when compared to DNA-based HPV tests. However this phenomenon has not yet been proven in HPV assays performing partial genotyping.

Design: The aim of our study was to compare the performance of DNA-based screening HPV assay Hybrid Capture 2 (detecting full spectrum of HR-HPVs) and its variant for HPV16/18/45 genotyping with mRNA-based screening HPV assay Aptima HPV (detecting full spectrum of HR-HPVs) and its variant for HPV16/18/45 genotyping, in residual liquid-based cytology samples of Czech women with histologically confirmed high-grade cervical lesions. Discordant samples were further genotyped using set of PCRs detecting three different genomic parts of HR-HPVs.

Results: In total, 117 samples were tested. Histological diagnoses included CIN2, CIN3, adenocarcinoma in situ, invasive carcinoma, and invasive adenocarcinoma. All but one sample were positive by both full spectrum HR-HPV tests. Using Hybrid capture 2 technique, 19 samples $(16.24 \%)$ were assigned false positive for the presence of HPV16/18/45 considering the cut-off value 1.00 RLU/CO, and 12 samples $(10.25 \%)$ considering the cut-off value 2.5 RLU/CO. The most common HPV type cross-reacting with specific HPV16/18/45 probe was HPV31.

Conclusions: Hybrid capture 2 test and Aptima HPV Assay have comparable characteristics for full spectrum HR-HPV detection in high-grade cervical lesions. However, Hybrid capture 2 assay for partial genotyping of HPV infection shows lower specificity for detection of HPV16/18/45 when compared to Aptima HPV 16 18/45 genotype assay.The most cross-reactive type is HPV31 probably due to its sequence similarity to HPV16.
1179 Inter-Observer Agreement for Measurement of Absolute Depth of Invasion in Endometrial Adenocarcinomas Using Light Microscopy and Whole Slide Scanning

Rossana Kazemimood, Andres Acosta, Farid Saei Hamedani, Shohreh Eliaszadeh, Rajyasree Emmadi, John Groth, Elizabeth Wiley. University of Illinois at Chicago, Chicago, IL.

Background: Measuring the depth of invasion in endometrial cancer is often a problematic task due to various artifacts and diverse morphological variations of the normal endo-myometrial junction. Also, the diagnosis of myometrial invasion (MI) in so-called superficially invasive endometrial carcinoma is poorly reproducible. In this study, we compare the degree of inter-observer agreement (IOA) on the diagnosis of MI and measurement of DOI, using conventional microscopy and digital slides.

Design: We selected forty slides with endometrial carcinoma from patients diagnosed at our institution. Glass slides were assigned a numerical code and scanned with the NanoZoomer 2.0-HT C9600 digital slide scanner (Hamamatsu Photonics KK, Japan). Digital slides were assigned a different code than the corresponding glass slide, and they were made accessible through web-based virtual microscopy software (Slidepath). Four participants were selected to evaluate MI and DOI on both the glass and virtual slides. An instructional set that included educational material on mimickers of MI, histologic features used to diagnose MI and directions on how to correctly measure DOI were provided to the four participants. Both MI and DOI were measured using a continuous scale and recorded in centimeters. IOA was evaluated using Cronbach's Alpha (SPSS Statistics for Windows, Version 20.0. Armonk, NY: IBM Corp.).

Results: Cronbach's Alpha for measurement of DOI in glass and virtual slides were 0.75 and 0.82 respectively. The Cronbach's Alpha for the diagnosis of MI was 0.65 on glass slides versus 0.75 on virtual slides.

Conclusions: Digital microscopy slightly improves IOA for the quantification of DOI using a continuous scale, and significantly improves IOA for the diagnosis of MI compared to glass slides. Unlike conventional microscopy, digital microscopy uses a continuous magnification scale and it allows for an easy spatial manipulation of the slide. These novel features can potentially increase IOA, and warrants further investigation in a larger group of observers.

\section{Her2-Neu Expression in Gastric-Type Endocervical} Adenocarcinomas

Zanobia Khan, Orit Freedman, Joerg Schwock, Marcus Bernardini, Amit Oza, Kathy Han, Marjan Rouzbahman. University Health Network, Toronto, ON, Canada; University of Toronto, Toronto, ON, Canada; Lakeridge Health Centre, Oshawa, ON, Canada.

Background: Gastric-type endocervical adenocarcinomas (GAS) are uncommon endocervical tumors and not associated with human papilloma virus (HPV) infection. They are clinically aggressive and present at a higher stage with propensity to metastasize to distant sites. Conventional treatment options are often not effective in controlling this tumor. Our objective was to explore the HER2 status and to review the clinicopathologic features of cases at our center.

Design: A retrospective review of 14 cases of GAS was conducted. Clinical and pathological parameters were recorded from the electronic patient record including: age, clinical presentation, treatment (tx), follow up (f/u), recurrence, tumor type, stage and lymph-vascular space invasion (LVSI). All available tumor slides were re-assessed by a gynecologic pathologist. Immunohistochemistry for p16 and p53, molecular testing for HPV and fluorescence in situ hybridization (FISH) for HER2 were performed.

Results: Mean age at diagnosis was 57.6 years (range; 35 to 79 ). The majority of patients $(8 / 14,57 \%)$ presented with advanced stage (stage II to IV). LVSI was present in $9 / 13$ $(69 \%)$ cases. 12 cases were negative for $\mathrm{p} 16,1$ showed focal staining and 1 was diffusely positive. Abnormal p53 expression was seen in 9 cases ( 3 diffuse overexpression, 6 null expression). 5 cases showed a wild-type p53 pattern. HPV DNA was not detected in any of the cases with tissue available for study $(0 / 12)$. HER2 amplification was reported in $4 / 12$ cases $(33 \%)$. Clinical f/u information was available in 13 cases. The mean f/u period was 31.1 months (range 4-95). 12/14 patients received adjuvant treatment (radiation + /- chemotherapy). 6/12 (50\%) died of disease and 6/12 (50\%) were alive with no evidence of disease at the time of last follow up. 3 of $4(75 \%)$ patients with HER2 amplification died of disease.

Conclusions: GAS are biologically aggressive tumors. Given the rarity of these tumors, recognition is extremely important for appropriate management of these patients. The HER 2 amplification in $33 \%$ of cases in our series is significant as it may have treatment implications. Although $75 \%$ of our patients with HER 2 amplification died of disease, the impact of HER2 amplification in outcome requires study in larger series.

\section{$1181 \quad$ Placental Lesions in Meconium Aspiration Syndrome}

Binnari Kim, Jung-sun Kim, Soo-young Oh. Samsung Medical Center, Sungkyunkwan University School of Medicine, Seoul, Korea.

Background: Meconium aspiration syndrome (MAS) is defined by respiratory distress requiring supplemental oxygen in a meconium-stained neonate. MAS is clinically subclassified as mild, moderate, and severe according to oxygen requirement, however severe MAS is suggested to be pathophysiologically distinct from mild or moderate MAS.

Design: The aim of this study is to compare histological findings in meconium-stained placentas from MAS neonates with those from non-MAS neonates and to analyze the correlation between the severity of MAS and the grade of the histological parameters. The 160 singleton term meconium stained placentas were included in this study. We used Statistical Package of Social Science (SPSS) to compare the parameters.

Results: MAS developed in 41 out of 160 cases. Funisitis $(p<0.01$ ), findings associated with maternal underperfusion and chronic deciduitis $(\mathrm{p}<0.05)$ were more frequent in 
the MAS. However, acute chorioamnionitis, amnion degeneration, meconium-laden macrophages, chorionic vascular muscle necrosis, and fetal thrombo-occlusive disease did not show significant differences between in the MAS and the non-MAS. There was a correlations between the degree of funisitis and the MAS severity $(\mathrm{p}<0.001)$. Chorionic vascular muscle necrosis also showed a correlation with the MAS severity $(\mathrm{p}<0.05)$. Conclusions: Fetal inflammatory response evidenced by funisitis occurs prenatally in MAS and the stage of funisitis and chorionic vascular muscle necrosis may be predictive markers for the severity of MAS.

\section{MED12 Mutation in Uterine Adenomyoma}

Masahiko Kito, Daichi Maeda, Yukihiro Terada, Akiteru Goto. Akita University, Akita, Japan.

Background: Adenomyoma of the uterus is a biphasic nodular lesion composed of a mesenchymal component with smooth muscle differentiation and glandular epithelium, most often of the endometrial type. Controversy remains over the nature of uterine adenomyoma. Some believe that the lesion is a nodular adenomyosis and thus non-neoplastic, while others recognize it as a true neoplasm. No previous study has assessed this issue from a genetic aspect. We speculated, based on our experience, that a subset of uterine adenomyomas is a true neoplasm: either a leiomyoma with entrapped endometrial glands or a distinct biphasic tumor. To verify this hypothesis, we examined uterine adenomyomas for MED 12 mutations, which are frequently detected in uterine leiomyomas $(70 \%)$ and mammary biphasic tumors $(60 \%$ in fibroadenomas and $80 \%$ in phyllodes tumors)

Design: A total of 16 cases of uterine adenomyomas were retrieved and assessed for clinicopathological factors. Immunohistochemistry for smooth muscle actin (SMA), desmin and CD10 was performed. Initially, exon 2 of MED12 was Sanger-sequenced using DNA obtained by macrodissection of the adenomyomas. For cases that were positive for somatic MED12 mutations, we then performed microdissection of the mesenchymal and epithelial components of the adenomyomas. The DNA extracted from each component was further analyzed for MED12 mutations.

Results: Of the 16 adenomyomas, eight were intramural, five were polypoid/ submucosal, and two were subserosal. Positive immunoreactivity for SMA and desmin was observed in the mesenchymal component in all lesions. CD10-positive stromal cells were observed around the glandular epithelium. MED12 mutations were detected in three adenomyomas $(3 / 16,19 \%)$, all in a known hot spot (codon 131). These MED12mutated adenomyomas were intramural, submucosal and subserosal. In all three lesions, MED12 mutations were detected in multiple spots of the mesenchymal component. The epithelial component did not harbor MED12 mutations.

Conclusions: $M E D 12$ is mutated in a subset of uterine adenomyomas, and the mutation is exclusively found in the mesenchymal component. The relatively low frequency of the mutation suggests that not all adeomyomas are leiomyomas with entrapped glands. However, some adenomyomas are true neoplasms, which may represent a variant of leiomyoma or a biphasic tumor with some analogy to mammary fibroadenoma.

\section{Morphological Reproducibility, Genotyping and Immunohistochemical Profiling Do Not Support a Category of Seromucinous Carcinoma of the Ovary}

Martin Kobel, Peter F Rambau, John B McIntyre, Jennifer Taylor, Sandra Lee, Travis Ogilvie, Anna Sienko, Don Morris, Maire Duggan, W Glenn McCluggage. University of Calgary, Calgary, AB, Canada; Tom Baker Cancer Centre, Calgary, AB, Canada; Belfast Health and Social Care Trust, Belfast, United Kingdom.

Background: The 2014 World Health Organization Classification of Tumors of the Female Reproductive Organs endorsed the category of seromucinous carcinoma, a neoplasm which exhibits morphological and immunophenotypic overlap with other histotypes of ovarian carcinoma. The objective of this study was to determine if seromucinous carcinoma is a distinct histotype by assessing its diagnostic agreement and comparing its molecular composition to the five major histotypes of ovarian carcinoma. Design: Thirty-two tumors diagnosed as seromucinous carcinomas from two centres were included. Eighteen cases were randomly selected for a review set which comprised a total of 50 ovarian carcinomas of various histotypes. Histotype by morphology was independently assessed by four pathologists. Histotype-specific immunophenoand genotype was then assigned to the 32 seromucinous carcinomas based on immunohistochemistry using a diagnostic 8-marker immunohistochemical panel, and targeted next generation sequencing (NGS) for somatic mutations in 59 genes which included recurrent mutations in ovarian carcinomas.

Results: There was low to modest agreement between pathologists with the reference diagnosis of seromucinous carcinoma, ranging from $39-56 \%$ for the four observers. The immunophenotype was not unique but overlapped predominantly with endometrioid carcinoma and to a lesser extent with low-grade serous carcinoma. The NGS detected 57 mutations. The most common mutations were KRAS (21/32, 66\%), PIK3CA (10/32, 31\%), ARIDIA (5/32, 16\%), and PTEN (4/32, 12\%); no CTNNB1 mutations were identified. Eight cases (25\%) harbored concurrent $K R A S / P I K 3 C A$ mutations. An endometrioid genotype was then assigned to 18 cases, a low-grade serous genotype to 10 , a mucinous genotype to 1 and 3 cases were uninformative.

Conclusions: The morphological diagnosis of seromucinous carcinoma is not reliable and it does not exhibit a distinct IHC phenotype or genotype. The molecular features overlap mostly with endometrioid and low-grade serous carcinomas. Our data suggests the category be discontinued as ancillary molecular tests can assign cases to one of the major histotypes of ovarian carcinoma. In this study, integrating morphology, IHC phenotype and genotyping resulted in reclassifying 32 seromucinous carcinomas to endometrioid in 23 cases (endometrioid carcinomas with mucinous differentiation), low-grade serous in 8 cases and mucinous in one case.
1184 TP53 Expression Status and Association with Outcome within Grade 3 Endometrioid Carcinomas of the Endometrium

Martin Kobel, Eshetu G Atenafu, Gregg S Nelson, Marcus Bernardini, Blaise Clarke. University of Calgary, Calgary, AB, Canada; Princess Margaret Cancer Centre, Toronto, ON, Canada; University of Toronto, Toronto, ON, Canada.

Background: High-grade carcinomas account for the majority of deaths from endometrial carcinomas. There have been issues with the reproducibility of histotype within the high-grade group and ancillary molecular tests are required for precise diagnosis. The Cancer Genome Atlas (TCGA) Project established four molecular subtypes for the endometrioid histotype. One of these, the copy number-high subtype, commonly harbors TP53 mutation and is associated with an unfavorable outcome. Despite underlying molecular differences, the TCGA lumped serous and endometrioid carcinomas together. We have recently shown that optimized immunohistochemistry is an accurate predictor of TP53 mutation status using ovarian carcinoma samples (J Path: Clin Res. doi:10.1002/cjp2.53). The objective of this study was to clarify whether TP53 abnormal endometrioid carcinomas grade 3 (EEC3) have a significantly shorter survival than TP53 wild type EEC3 or whether the TCGA finding was driven by endometrial serous carcinomas (ESC).

Design: Tissue microarrays from two studies from the CHREC (Canadian High Risk Endometrial Cancer) consortium were included in the study. 270 EEC3 and ESC were assessed for abnormal or normal TP53 expression by immunohistochemistry and univariate Kaplan-Meier survival analysis was performed.

Results: TP53 abnormal EEC3 had a significantly higher risk of death $(\mathrm{HR}=2.08,95 \%$ CI $1.07-4.03, \mathrm{p}=0.030$ ) compared to TP53 normal EEC3 and no significant difference was observed between TP53 abnormal EEC3 and ESC.

Conclusions: Our results suggest that TP53 abnormal EEC 3 behave more aggressively similar to ESC supporting the judicious use of TP53 immunohistochemistry and that TP53 status should be considered in management of these tumors.

1185 Molecular Profiling and Morphologic Correlation as a Quality Assurance Metric in Epithelial Ovarian Cancer

David Kolin, Tracy Stockley, Suzanne Kamel-Reid, Swati Garg, Amit Oza, Stéphanie Lheureux, Helen MacKay, Marcus Butler, Neesha Dhani, Blaise Clarke, Patricia Shaw. University Health Network, Toronto, ON, Canada.

Background: Ovarian cancer is a morphologically and genetically heterogenous disease. Targeted gene sequencing of solid tumors is increasingly requested by oncologists to detect actionable mutations. However, sequencing also provides useful diagnostic information to pathologists. The purpose of this study was to correlate molecular features of ovarian cancer with tumor histotype, and to examine the utility of molecular diagnostics as a quality control mechanism.

Design: Patients with advanced ovarian carcinoma were consented as part of an institutional molecular profiling program. Representative H\&E sections from formalinfixed paraffin embedded (FFPE) tissue were reviewed and DNA extracted from selected tumor-rich regions. Molecular profiling was performed using the Illumina TruSeq Amplicon Cancer Panel (167 cases, regions of 48 genes) or Ion AmpliSeq Cancer Hotspot Panel (5 cases, regions of 50 genes). Some cases were tested for recurrent hotspot mutations by a MADLI-TOF custom Sequenom panel (43 cases, recurrent mutations of 23 genes).

Results: 217 ovarian malignancies were sequenced. A mutation was found in the majority of cases $(67 \%)$, and was detected when the tumor comprised as little as $20 \%$ of the sample. Mutation detection was limited in post-chemotherapy tissues with scant tumor cellularity. Mutations were detected in 52\% of 46 cases of low-grade serous carcinoma. Clear cell carcinomas frequently showed PIK3CA mutations (61\% of 18 cases). Only $75 \%$ of the 114 cases of high-grade serous carcinoma (HGSC) had a detected TP53 mutation, lower than expected due in part to the limitations of the panel testing. In cases with p53 immunohistochemistry available, all cases of HGSC (including those with no mutation detected) showed an aberrant pattern of staining. Review of discordant sequencing and morphology resulted in a revised diagnosis in 2 cases (HGSC to LGSC and endometrioid to HGSC).

Conclusions: Next generation sequencing using targeted panels can be successfully performed on archival FFPE tissue. Cases with discordant molecular and morphologic impressions should trigger a review of both histopathology and variant analysis. The absence of a detected mutation in a tumor may be due to tumor biology, insufficient tumor cellularity, or sequencing limitations. Molecular studies are best interpreted in the context of tumor morphology and classification inclusive of immunohistochemistry.

1186 Patterns of Recurrences in Grade 1 Endometrioid Endometrial Adenocarcinoma (EEC)

Melissa Krystel-Whittemore, Esther Oliva. Massachusetts General Hospital, Boston, MA.

Background: EEC is the most common type of endometrial cancer. Patients with grade 1 (G1) have better survival than G3 EEC (95\% vs 68\%). However, a subset of G1 EEC recur and implicated factors are not well-known. These G1 EEC appear to have differences based on patterns of recurrence (vagina vs others), with vaginal recurrences associated with less aggressive tumors.

Design: We identified patients with G1 EEC between 2009 and 2012 and reviewed clinicopathological characteristics and outcome.

Results: 13 patients with G1 EEC developed recurrences, with a median disease free interval of 27 months. Median age was 68 (54-78) years. All were treated by hysterectomy, bilateral salpingo-oophorectomy and complete staging. Tumor median size was $3.9(1.1-10) \mathrm{cm}$. Architecture consisted of predominately back to back glands in 11 tumors. Papillary architecture and fused glands with cribriforming were predominant in one tumor each. Squamous differentiation was noted in 4, mucinous 
differentiation in 5, and non-villous papillae in 1 tumors. All EEC had bland nuclear features. Comedonecrosis was seen in one tumor. Pattern of invasion was destructive in 5, bulbous in 2, and pushing in 1 tumor; it was not applicable in 5, as they invaded $<1 \mathrm{~mm}$. 4 EEC had lower uterine segment (LUS) involvement and 1 lymphovascular invasion (LVI). 6 out of 13 patients developed vaginal recurrences, 2 had pelvic recurrences (one with colonic involvement), 2 recurred in the omentum, and 1 each in pelvic lymph nodes, lungs and abdominal wall. 12 patients are alive with disease and 1 died of disease (lung metastases). Correlation between histologic parameters and vaginal vs other recurrences is shown in Table 1.

\begin{tabular}{|l|l|l|}
\hline & Vaginal recurrences $(\mathrm{n}=6)$ & Non-vaginal recurrences $(\mathrm{n}=7)$ \\
\hline Age & 73 & 63 \\
\hline Size & 4.6 & 3.1 \\
\hline Glandular architecture & $5 / 6$ & $6 / 7$ \\
\hline Invasion: Destructive & $1 / 6$ & $4 / 7$ \\
MELF & $0 / 6$ & $1 / 7$ \\
Bulbous & $1 / 6$ & $1 / 7$ \\
$<1 \mathrm{~mm}$ & $3 / 6$ & $2 / 7$ \\
\hline Comedonecrosis & $0 / 6$ & $1 / 7$ \\
\hline Brisk apoptoses & $3 / 6$ & $2 / 7$ \\
\hline Mitoses/10 HPF & 5.2 & 5.6 \\
\hline LVI & $0 / 6$ & $1 / 7$ \\
\hline LUS + & $3 / 6$ & $1 / 7$ \\
\hline
\end{tabular}

Conclusions: As recently reported in the literature, a destructive pattern of invasion in G1 EEC from patients with non-vaginal recurrences was more common (4/7; one died of disease) as compared to those with vaginal recurrences (1/6). Other reported parameters including LVI, MELF pattern of invasion, and comedonecrosis were also noted in EEC from patients with non-vaginal recurrences, although as isolated cases. These findings suggest that EEC in patients with vaginal recurrences are less aggressive and may have a different molecular landscape.

1187 Molecular Modifiers of the Tumor Immune Microenvironment: Activation of the $\mathrm{Wnt} / \beta$-Catenin Pathway Is Associated with Local Immunosuppression in Endometrial Cancer

Katherine Kurnit, Mark Munsell, Russell R Broaddus. MDACC, Houston, TX.

Background: We have previously shown that activation of the Wnt/ $\beta$-catenin pathway and $C T N N B 1$ (gene encoding $\beta$-catenin) mutation are associated with decreased survival in low grade, early stage endometrioid endometrial cancer. Suppression of local tumor immunity is one potential mechanism to explain these findings. We hypothesized that activation of the Wnt/ $\beta$-catenin pathway is associated with increased tumor levels of the immunosuppressive cytokines IL-10 and TGF- $\beta 2$.

Design: qRT-PCR for $T G F-\beta 2$ and $I L-10$ was performed on 169 frozen endometria carcinomas ( $82 \%$ endometrioid, $18 \%$ mixed endometrioid and non-endometrioid). Results were expressed as molecules of transcript/molecules $18 S \mathrm{rRNA}$. Activation of the $\mathrm{Wnt} / \beta$-catenin pathway was assessed by $C T N N B I$ gene sequencing and quantification of transcript levels for Cyclin D1, with presence of $C T N N B 1$ mutation and/or high levels of Cyclin DI indicative of pathway activation. Descriptive statistics, Fisher's exact test, Wilcoxon rank sum test, and Spearman's rank correlation were used.

Results: Activation of the Wnt/ $\beta$-catenin pathway, as judged by high tumor levels of Cyclin Dl, was associated with younger age at diagnosis but no other clinical or pathological characteristics. Higher Cyclin DI levels correlated with higher levels of both $T G F-\beta 2$ and $I L-10$. CTNNBI mutation was associated with significantly higher levels of $T G F-\beta 2$ compared to wildtype tumors (105.0 vs. $2.1 \times 10^{-6}, p=0.02$ ). $T G F-\beta 2$ was also significantly higher in low grade tumors, as well as in endometrioid compared with mixed histology tumors. $I L-10$ levels were not associated with $C T N N B 1$ mutation or any clinical or pathological parameter.

Table 1. Baseline characteristics for the entire cohort and stratified across high and low Cyclin DI levels.

\begin{tabular}{|c|c|c|c|c|}
\hline Characteristic & Overall & $\begin{array}{c}\text { Low Cyclin D1 } \\
<452 \times 10^{-6}\end{array}$ & $\begin{array}{l}\text { High Cyclin D1 } \\
\geq 452 \times 10^{-6}\end{array}$ & $p$-value \\
\hline Age at diagnosis (median), years & 59 & 62.5 & 57 & 0.01 \\
\hline BMI (median), $\mathrm{kg} / \mathrm{m}^{2}$ & 35.4 & 37.6 & 33.5 & 0.21 \\
\hline $\begin{array}{ll}\text { Histology, n (\%) } & \begin{array}{l}\text { Endometrioid } \\
\text { Mixed }\end{array}\end{array}$ & $\begin{array}{c}138(81.7 \%) \\
30(17.8 \%)\end{array}$ & $\begin{array}{l}65(84.4 \%) \\
12(15.6 \%)\end{array}$ & $\begin{array}{l}70(80.5 \%) \\
17(19.5 \%)\end{array}$ & 0.54 \\
\hline Grade, n (\%) & $\begin{array}{l}18(10.7 \%) \\
96(56.8 \%) \\
52(30.8 \%)\end{array}$ & $\begin{array}{l}9(11.5 \%) \\
48(61.5 \%) \\
21(26.9 \%)\end{array}$ & $\begin{array}{l}9(10.7 \%) \\
45(53.6 \%) \\
30(35.7 \%)\end{array}$ & 0.51 \\
\hline Stage, n (\%) & $\begin{array}{c}114(67.5 \%) \\
6(3.6 \%) \\
32(18.9 \%) \\
15(8.9 \%)\end{array}$ & $\begin{array}{c}54(70.1 \%) \\
2(2.6 \%) \\
15(19.5 \%) \\
6(7.8 \%)\end{array}$ & $\begin{array}{c}58(67.4 \%) \\
4(4.7 \%) \\
16(18.6 \%) \\
8(9.3 \%)\end{array}$ & 0.91 \\
\hline Tumor size (median), $\mathrm{cm}$ & 4.5 & 5 & 4.55 & 0.98 \\
\hline $\begin{aligned} \text { Myometrial invasion, } \mathrm{n} \mathrm{( \% )} & \\
& <50 \% \\
& \geq 50 \%\end{aligned}$ & $\begin{array}{c}112(66.3 \%) \\
57(33.7 \%)\end{array}$ & $\begin{array}{l}48(61.5 \%) \\
30(38.5 \%)\end{array}$ & $\begin{array}{l}61(70.1 \%) \\
26(29.9 \%)\end{array}$ & 0.25 \\
\hline LVSI, n (\%) & $\begin{array}{l}92(54.4 \%) \\
67(39.6 \%)\end{array}$ & $\begin{array}{l}43(59.7 \%) \\
29(40.3 \%)\end{array}$ & $\begin{array}{l}47(56.6 \%) \\
36(43.4 \%)\end{array}$ & 0.75 \\
\hline $\begin{array}{r}\text { TGF- } \beta 2\left(\text { median, } \times 10^{-6}\right) \\
\text { Endometrioid }\end{array}$ & $\begin{array}{l}19.2 \\
56.1\end{array}$ & $\begin{array}{c}0 \\
8.8\end{array}$ & $\begin{array}{l}105.0 \\
126.1\end{array}$ & $\begin{array}{l}<0.001 \\
<0.0001\end{array}$ \\
\hline $\begin{array}{r}I L-10\left(\text { median, } \times 10^{-6}\right) \\
\text { Endometrioid }\end{array}$ & $\begin{array}{l}29.1 \\
28.3\end{array}$ & $\begin{array}{l}21.6 \\
20.7\end{array}$ & $\begin{array}{l}36.2 \\
37.7\end{array}$ & $\begin{array}{l}0.009 \\
0.003\end{array}$ \\
\hline
\end{tabular}

Conclusions: Endometrial carcinomas with activation of the Wnt/ $\beta$-catenin pathway show evidence of microenvironment immunosuppression. Moving forward, a targeted therapeutic for the Wnt/ $\beta$-catenin pathway in conjunction with immunotherapy may be an effective combination for treating recurrence in this group of endometrial cancer patients.

$1188 \quad$ p53 Aberrant Endometrial Carcinomas with Loss of Staining for PTEN and/or Mismatch Repair Proteins: A Clinicopathologic Analysis Nicholas Ladwig, Karuna Garg. University of California - San Francisco, San Francisco, CA.

Background: Endometrial carcinomas (EC) that show aberrant staining for p53 are typically diagnosed as serous carcinoma and are associated with poor clinical outcome. The endometrial Cancer Genome Atlas (TCGA) data suggests that in a subset of ECs, the p53 mutation may be secondary to a hypermutated (due to mismatch repair or MMR abnormality) or ultramutated (due to POLE mutation) tumor profile and is not the driver mutation and hence may not carry the same clinical significance. Therefore such tumors should have good/better clinical outcomes in spite of the aberrant p53 staining pattern. The POLE mutated tumors also frequently harbor mutations in PTEN With this background, the aim of our study was to assess the frequency of abnormal staining for PTEN and MMR in EC with aberrant p53 IHC and to correlated the staining pattern with clinical outcome.

Design: Cases of EC with aberrant p53 staining were selected. Following morphologic review, IHC stains for PTEN, p16, MLH1, PMS2, MSH2, and MSH6 were performed and analyzed. Clinical data was collected from EMR.

Results: We identified 31 cases which were divided into 4 groups based on their IHC profile.

\begin{tabular}{|c|c|c|c|c|c|c|}
\hline p53 & p16 & PTEN & MMR & Age & Stage & Status (years from diagnosis) \\
\hline \multicolumn{7}{|c|}{ Group $1(\mathrm{n}=5)$ : p53 Aberrant, PTEN Lost, MMR Retained } \\
\hline A & + & $\mathrm{L}$ & $\mathrm{R}$ & 63 & IVB & LTF \\
\hline A & + & $\mathrm{L}$ & $\mathrm{R}$ & 75 & IIIC & DOD $(0.7)$ \\
\hline A & + & $\mathrm{L}$ & $\mathrm{R}$ & 60 & IIIA & NED (3.2) \\
\hline A & + & $\mathrm{L}$ & $\mathrm{R}$ & 40 & IVA & NED (13.3) \\
\hline A & + & $\mathrm{L}$ & $\mathrm{R}$ & 76 & IA & NED (3.6) \\
\hline \multicolumn{7}{|c|}{ Group 2 ( $\mathrm{n}=1$ ): p53 Aberrant, PTEN Retained, MMR Lost } \\
\hline A & + & $\mathrm{R}$ & $\begin{array}{c}\mathrm{L} \\
\text { (MSH6) }\end{array}$ & 6 & IB & NED (4.7) \\
\hline \multicolumn{7}{|c|}{ Group 3 ( $\mathrm{n}=1)$ : p53 Aberrant, PTEN Lost, MMR Lost } \\
\hline A & - & $\mathrm{L}$ & $\begin{array}{c}\mathrm{L} \\
\text { (PMS2) }\end{array}$ & 62 & IIA & NED (7.3) \\
\hline \multicolumn{7}{|c|}{ Group $4(\mathrm{n}=24)$ : $\mathrm{p} 53$ Aberrant, PTEN Retained, MMR Retained } \\
\hline A & + & $\mathrm{R}$ & $\mathrm{R}$ & $68(41-87)$ & $\begin{array}{l}\text { I: } 9 \\
\text { II: } 10 \\
\text { III-IV: } 15\end{array}$ & $\begin{array}{c}\text { NED: } 6(8.6) \\
\text { AWD: } 5(2.3) \\
\text { DOD: } 6(2) \\
\text { LTF: } 7\end{array}$ \\
\hline
\end{tabular}

Key:

$\mathrm{A}=$ aberrant

$\mathrm{L}=$ lost

$\mathrm{R}=$ retained

LTF $=$ lost to follow up

$\mathrm{AWD}=$ alive with disease

$\mathrm{DOD}=$ dead of disease

NED $=$ no evidence of disease

Conclusions: In our small preliminary data set, we found PTEN loss in 5 (16\%), MMR loss in $1(3 \%)$ and loss of both PTEN and MMR in $1(3 \%)$ of 31 p53 aberrant EC. Although the numbers are small (we are currently accumulating more cases to expand this dataset), our data suggests that p 53 aberrant EC with PTEN and/or MMR loss may have better clinical outcomes and raises the possibility that PTEN and MMR IHC may serve as helpful prognostic markers in this setting.

$1189 \quad$ Uterine Inflammatory Myofibroblastic Tumors Frequently Harbor IGFBP5-ALK and THBS1-ALK Fusions

Cheng-Han Lee, Josh Haimes, Brian Kudlow, Jen-Chieh Lee, W Glenn McCluggage, Tony Ng, Colin J Stewart. Vancouver General Hospital and BC Cancer Agency, Vancouver, Canada; ArcherDX, Inc, Boulder, CO; National Taiwan University Hospital, Taiepi, Taiwan; Belfast Health and Social Care Trust, Belfast, United Kingdom; King Edward Memorial Hospital, Perth, Australia.

Background: Inflammatory myofibroblastic tumor (IMT) can occur in a number of anatomic sites, including the uterus. Like its soft tissue counterpart, uterine IMT frequently expresses ALK and shows $A L K$ genetic rearrangement. The aim of this study is to fully characterize the genetic fusions that occur in uterine IMT as well as its histologic mimics.

Design: We identified 10 uterine IMTs with classic histology and 8 myxoid uterine smooth muscle tumors (6 leiomyomas, 1 STUMP and 1 leiomyosarcoma) in which the differential of IMT was considered. A next generation sequencing-based fusion assay (ArcherDx FusionPlex Comprehensive Thyroid Lung panel) was used to detect genetic fusions involving $A L K$, ROS1, RET and NTRK1/2/3.

Results: ALK was expressed immunohistochemically in 9 of 10 IMTs and 1 of 8 myxoid smooth muscle tumors (a myxoid leiomyoma). Fusion transcripts involving $A L K$ were identified in all 9 ALK immuno-positive IMTs, with 3 harboring IGFBP5-ALK, 3 harboring THBS1-ALK, 2 harboring $F N 1-A L K$ and 1 harboring TIMP3-ALK. The single ALK immuno-negative IMT lacked any demonstrable kinase fusions. Among the 8 
myxoid uterine smooth muscle tumors, IGFBP5-ALK fusion transcript was identified in the ALK immuno-positive myxoid leiomyoma; no fusion transcripts were identified in the other myxoid smooth muscle tumors examined. Further review revealed that while a diagnosis of IMT was considered for the ALK immuno-positive myxoid leiomyoma, this diagnosis was not rendered because fluorescence in situ hybridization (FISH) was interpreted as being negative for $A L K$ genetic rearrangement.

Conclusions: Our findings confirm the high frequency of $A L K$ fusions in uterine IMT and demonstrate an enrichment of novel 5' $A L K$ fusion partners in IGFBP5 and THBSI for IMTs at this site. Given that IGFBP5 and $A L K$ are both located on chromosome 2, FISH analysis for $A L K$ rearrangement may not be reliable and a negative result should not dissuade against a diagnosis of uterine IMT if the histologic and ALK immunostaining findings are supportive.

1190 Clinical Targeted Next-Generation Sequencing Shows Increased Mutational Load in Endometrial Adenocarcinoma with Deficient DNA Mismatch Repair

Paul Lee, Eric Duncavage, Samantha McNulty, Jonathan W Heusel, Ian Hagemann. Washington University, St. Louis, MO.

Background: A subset of endometrial adenocarcinomas (EAC) exhibit microsatellite instability and have deficient DNA mismatch repair (dMMR). The overall aim of the study was to compare the spectrum of mutations in EAC with and without dMMR by clinical targeted next-generation sequencing.

Design: We retrospectively identified 12 EAC with known MMR status that underwent targeted clinical next-generation sequencing of a panel of 164 cancer-related genes. The MMR status was ascertained by immunohistochemistry for MLH1, PMS2, MSH2, and MSH6. Histologic and clinical data were extracted from clinical records. We compared somatic mutations in EAC with deficient MMR (dMMR) against cases with proficient MMR (pMMR)

Results: The dMMR and pMMR cases did not significantly differ for FIGO grade or clinical stage. The dMMR EAC showed a mean of 32.3 mutations per case while pMMR EAC had an average of 14.8 ( $\mathrm{p}=0.055$ by 2 -tailed $\mathrm{t}$-test). The most commonly mutated genes were PTEN (100\% of dMMR, $75 \%$ of pMMR), PIK3CA ( $75 \%$ vs. $75 \%$ ), ATM ( $88 \%$ vs. $25 \%$ ), and FLT3 (63\% vs. $50 \%$ ) respectively.

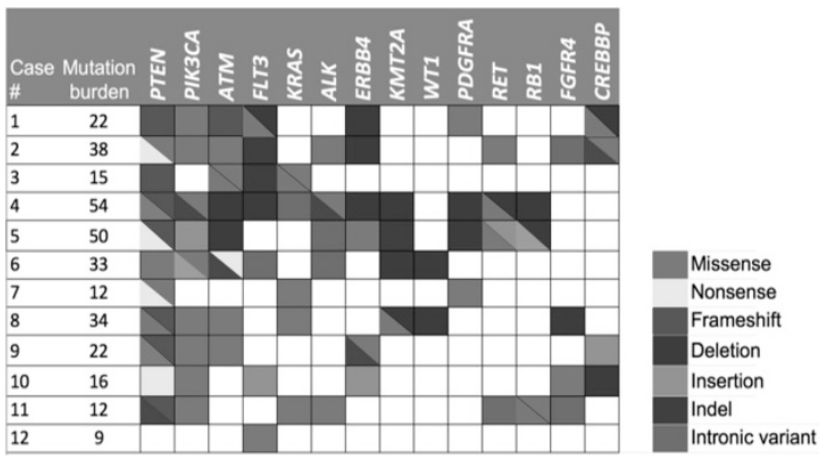

Transitions were more common in the dMMR group as compared to the pMMR cohort, with transitions/transversions $=4.2$ vs. 1.5 respectively $(\mathrm{p}=0.054$ by 2 tailed t-test). The dMMR and pMMR cohorts did not differ for frameshift-to-inframe mutation ratio or proportion of substitution/deletions/insertion. The dMMR group demonstrated more copy number variant gains than losses. The gains were mainly identified in chromosomes $1 \mathrm{q}$ and $8 \mathrm{q}$.

Conclusions: Tumorigenesis of EAC in the context of dMMR demonstrated heavier mutational burdens, more transition mutations, and unique copy number alterations. This spectrum of genetic alterations can be identified using NGS data captured from a targeted cancer gene panel. The increased mutation load in dMMR cases may provide increased numbers of actionable targets or neoantigens for adoptive immunotherapy.

1191 Tissue-Based Immunohistochemical Biomarker Expression in Glandular Malignancies of the Cervix: A Systematic Review and MetaAnalysis

Sandra Lee, Vikrant Sahasrabuddhe, Diana Mendoza-Cervantes, Sarah Rose, Rachel Zhao, Maire Duggan. South Health Campus, Calgary, AB, Canada; National Cancer Institute, Bethesda, MD; University of Calgary, Calgary, AB, Canada.

Background: Immunohistochemistry is widely used to support a diagnosis of cervical glandular malignancy. This systematic review and meta-analysis (SRMA) was performed to investigate biomarker expression in premalignant and malignant glandular histotypes.

Design: The SRMA used a PICOT framework and QUADAS-2 to evaluate study quality. The literature search spanned 40 years and ended June 30, 2015. Abstracts of identified records were independently screened by 2 of the authors who conducted a full text review of selected articles. Of 902 abstracts screened, 154 articles were selected for full review. 25 articles were included. No biomarker had sufficient studies for a MA. Biomarker positivity percentages in samples across studies were pooled to develop a combined estimate for each biomarker in each histotype. To examine if patterns of biomarker positivity differed between cases and comparators, an analytical framework of unsupervised hierarchical clustering, with complete linkage and a Euclidian distance metric (Cluster 3.0 open access software) was applied. Patterns were simultaneously clustered and visualized via dendrograms (Java TreeView software).
Results: 7 of 19 biomarkers expressed in AIS and 3 of 53 expressed in mucinous adenocarcinoma showed almost a $50 \%$ difference in expression compared to other premalignant and malignant histotypes (Table 1).

\begin{tabular}{|l|l|}
\hline AIS vsHSIL & NoneCA-IX $(1.00$ vs 0.16$)$, p16 $(0.90$ vs \\
Atypical LEGH & $0.13)$ \\
Mucinous adenocarcinomaEndometrioid & Alpha-SMA $(0.00$ vs 1.00$)$, Pax8 $(1.00$ vs \\
adenocarcinoma & $0.50)$ CEA $(0.00$ vs 0.86$)$ \\
Adenosquamous carcinomaMDA/gastric type & VIL1 $(0.80$ vs 0.20$)$ Alpha SMA $(0.00$ vs \\
adenocarcinoma & $1.00)$, CEA $(0.00$ vs 0.89$)$, HIK1083 $(0.19$ vs \\
& $0.75)$, p16 $(0.90$ vs 0.30$)$, p53 $(0.00$ vs 0.67$)$ \\
\hline Mucinous adenocarcioma vsEndometrioid & NoneER $(0.76$ vs 0.00$)$ \\
adenocarcinomaAdenosquamous carcinoma & HIK1083 $(0.22$ vs 0.76$), \mathrm{p} 16(0.88$ vs 0.28$)$, \\
MDA/gastric type adenocarcinomaMesonephric & Vimentin $(0.49$ vs 1.00$)$ CD $10(0.06$ vs 0.67$)$, \\
carcinoma & Calretinin $(0.17$ vs 0.67$)$ \\
Serous and Clear cell carcinoma & CEA $(0.69$ vs 0.00$)$ \\
\hline
\end{tabular}

Conclusions: Biomarker expression in cervical glandular malignancies is understudied. This SRMA identified 1 or more of 12 biomarkers worth additional investigation as useful discriminants of malignant glandular histotypes from each other.

1192 Endometrial Cancer PD-L1 Expression Is Associated with Mismatch Repair Deficiency, Lymphovascular Invasion and Lymph Node Metastases

Zaibo Li, Amy S Joehlin-Price, Martins Ayoola-Adeola, Karin Miller, Anil Parwani, Floor J Backes, Ashley Felix, Adrian Suarez. The Ohio State University, Columbus, $\mathrm{OH}$; University of California, San Francisco, San Francisco, CA; Johns Hopkins University, Baltimore, MD.

Background: Programmed death 1 ligand 1 (PD-L1) is an immune regulatory molecule that limits antitumor immune activity. PD-L1 may be of particular importance for microsatellite unstable tumors that may express multiple neoepitopes as a result of their increased mutational burden.

Design: Immunohistochemistry for PD-L1 was performed on sections of a triple-core tissue microarray including 705 endometrial carcinomas (EC). PD-L1 staining was scored with a positive threshold of staining in $1 \%$ of tumor cells or immune cells. Logistic regression was used to evaluate odds ratios (ORs) and $95 \%$ confidence intervals (CIs) for associations between PD-L1 expression, clinicopathologic features and mismatch repair (MMR) protein expression.

Results: PD-L1 expressionin tumor cells or immune cells was observed in $7.4 \%$ $(52 / 705)$ of cases. Tumors characterized by absence of MSH2 and/or MSH6 protein expression $(\mathrm{OR}=20.15,95 \% \mathrm{CI}=7.39-54.92)$ or absence of MLH1 and/or PMS2 protein expression $(\mathrm{OR}=17.55,95 \% \mathrm{CI}=8.28-37.23)$ were significantly associated with PD-L1 expression in age-adjusted logistic regression models $(p<0.0001)$. Similarly, PD-L1 expression was associated with lymphovascular invasion $(\mathrm{p}=0.02)$ and lymph node metastases ( $\mathrm{pN} 1$ or $\mathrm{pN} 2, \mathrm{p}=0.02)$.

\begin{tabular}{|c|c|c|c|c|c|}
\hline & \multicolumn{2}{|c|}{ Negative $(n=653)$} & \multicolumn{2}{|c|}{ Positive $(n=52)$} & \multirow[b]{2}{*}{$\mathrm{p}^{*}$} \\
\hline & $\mathrm{n}$ & $\%$ & $\mathrm{n}$ & $\%$ & \\
\hline Age & & & & & 0.78 \\
\hline$<55$ & 177 & 27.1 & 12 & 23.1 & \\
\hline $55-64$ & 250 & 38.3 & 22 & 42.3 & \\
\hline$\geq 65$ & 226 & 34.6 & 18 & 34.6 & \\
\hline BMI & & & & & 0.04 \\
\hline Normal weight $(<25 \mathrm{~kg} / \mathrm{m2})$ & 68 & 10.4 & 4 & 7.7 & \\
\hline Overweight $(25-29.9 \mathrm{~kg} / \mathrm{m} 2)$ & 102 & 15.6 & 16 & 30.8 & \\
\hline Class I Obese $(30-34.9 \mathrm{~kg} / \mathrm{m} 2)$ & 131 & 20.1 & 10 & 19.2 & \\
\hline Class II Obese ( $35-39.9 \mathrm{~kg} / \mathrm{m} 2)$ & 104 & 15.9 & 10 & 19.2 & \\
\hline Class III Obese $(\geq 40 \mathrm{~kg} / \mathrm{m} 2)$ & 248 & 38.0 & 12 & 23.1 & \\
\hline Histology & & & & & 0.03 \\
\hline Low-grade endometrioid adenocarcinoma & 520 & 79.6 & 34 & 65.4 & \\
\hline High-grade endometrioid adenocarcinoma & 34 & 5.2 & 5 & 9.4 & \\
\hline Serous carcinoma & 37 & 5.7 & 5 & 9.6 & \\
\hline Carcinosarcoma & $\frac{\pi}{23}$ & 3.5 & $\frac{5}{1}$ & 1.6 & \\
\hline Mixed epithelial carcinoma & 27 & 4.1 & 3 & 5.8 & \\
\hline Other & 12 & 1.8 & 4 & 7.7 & \\
\hline Lymphovascular space invasion & & & & & 0.02 \\
\hline No & 488 & 74.7 & 31 & 59.6 & \\
\hline Yes & 164 & 25.1 & $\frac{31}{21}$ & 40.4 & \\
\hline TNM stage $(\mathrm{T})$ & & & & & 0.87 \\
\hline pT1a or T1b & 543 & 83.2 & 43 & 82.7 & \\
\hline pT2 & 37 & 5.7 & 2 & 3.8 & \\
\hline pT3 or pT4 & 73 & 11.2 & $\frac{2}{6}$ & 115 & \\
\hline TNM stage $(\mathrm{N})$ & & & & & 0.02 \\
\hline pNO & 374 & 57.3 & 28 & 53.8 & \\
\hline $\mathrm{pN1}$ & 40 & 6.1 & 8 & 15.4 & \\
\hline $\mathrm{pN} 2$ & 24 & 3.7 & 4 & 7.7 & \\
\hline $\mathrm{pNX}$ & 214 & 32.8 & 12 & 23.1 & \\
\hline MMR status & & & & & $<0.0001$ \\
\hline All proteins present & 526 & 81.0 & 10 & 19.6 & \\
\hline MSH2 or MSH6 absent & 25 & 3.9 & 9 & 17.6 & \\
\hline PMS2 absent and MLH1 present & 3 & 0.5 & 1 & 2.0 & \\
\hline MLH1 absent and PMS2 absent & 95 & 14.6 & 31 & 60.8 & \\
\hline
\end{tabular}

Conclusions: PD-L1 expression is seen only in a minority of EC and is strongly associated with MMR protein loss. PD-L1 expression may also correlate with clinicopathological data associated with more aggressive EC. PD-L1 targeted treatment may be of promise in EC. 
1193 PD-L1 Expression in Vulvar Squamous Cell Carcinoma: Immunohistochemical and RNA-Based In Situ Hybridization Analysis

Li Liang, Pamela Villalobos, Chi Hyun Lee, JIng Ning, Barbara Mino, Wei-Lien Wang, Jaime Rodriguez Canales, Amir A Jazaeri, Ignacio I Wistuba, Anna Yemelyanova. The University of Texas MD Anderson Cancer Center, Houston, TX.

Background: Immunotherapy with anti-PD1/PD-L1 agents has shown significant responses in solid tumors of various sites and histologies. Immunohistochemistry (IHC) for PD-L1 expression is being used to predict the response to therapy. Remarkably low thresholds of tumor cells staining (as low as $\geq 1 \%$ ) have been generally used to score tumors as positive. The data regarding PD-L1 expression in SCC of lower anogenital tract is relatively limited. The goal of this study is to evaluate PD-L1 expression in vulvar SCCs comparing immunohistochemical and mRNA-based in situ hybridization (ISH) analysis.

Design: Forty-three cases of vulvar SCC were selected. The tumors were classified as HPV-related and HPV-unrelated based on expression of p16. PD-L1 expression was evaluated by IHC using 2 antibodies PD-L1 28-8 and PD-L1 22C3 (Dako) in al cases and by RNA-based ISH (RNAscope, Advanced Cell Diagnostics) in selected 20 cases. For PD-L1 IHC, membranous staining in tumors cells was estimated to the nearest $10 \%(1-10 \%$ expression was scored as $10 \%)$; staining in the peritumoral stromal inflammatory infiltrate was recorded as positive or negative. ISH results were recorded as follows: 0 - negative, $1+$ rare punctate cytoplasmic signals in a few foci; $2+$ multiple cytoplasmic signals in multiple foci in tumor cells. Expression in the peritumoral stromal inflammatory infiltrate was also recorded as positive or negative. Statistical analysis was performed using $\mathrm{R}$ software (version 3.3.1).

Results: The study included 20 HPV-related SCCs (p16 +) and 23 HPV-unrelated tumors. The tumor cells were positive in $25(58 \%)$ and $24(56 \%)$ cases with anti-PD-L1 $28-8$ and $22 \mathrm{C} 3$ respectively. The percent of positive tumor cells ranged from 10-80 $\%$ (mean 20\% [28-8] and $19 \%$ [22C3], median $10 \%$ ). 20 cases were evaluated by ISH, all but one had positive signals in tumor cells; 13 cases had $1+$ and 6 cases, $2+$ expression. All cases had some expression in peritumoral stroma by ISH and all but one case by IHC. There was a strong correlation between IHC expression assayed by two antibodies $(\mathrm{p}<0.01)$ and between ISH and 28-8 ( $\mathrm{p}=0.01)$; ISH and $22 \mathrm{C} 3(\mathrm{p}=0.05)$ There were no statistically significant differences in PD-L1 expression between HPVrelated and HPV-unrelated SCCs.

Conclusions: PD-L1 expression is present in a subset of vulvar SCCs and appears independent of HPV status. Anti-PD-L1 antibody clones 28-8 and 22C3 perform similarly and correlate with ISH results. Further studies are required to determine the relevance of these findings for predicting response to immunotherapy.

\section{Epidermal Growth Factor Receptor (EGFR) Expression in Vulvar} Squamous Cell Carcinoma

Li Liang, Pamela Villalobos, Chi Hyun Lee, JIng Ning, Barbara Mino, Wei-Lien Wang, Jaime Rodriguez Canales, Amir A Jazaeri, Ignacio I Wistuba, Anna Yemelyanova. The University of Texas MD Anderson Cancer Center, Houston, TX.

Background: Vulvar squamous cell carcinoma (SCC) develops through two etiologically distinct pathways. One is related to high risk HPV infection and the other is HPV-unrelated and is thought to be associated with TP53 mutations. While some cases are cured with surgery with or without radiotherapy, the recurrence rates reach $24 \%$. Treatment of recurrent vulvar cancer includes surgery, chemotherapy and radiation therapy. EGFR overexpression and/or amplification in vulvar SCC have been linked to the depth of invasion, lymph node metastasis and tumor recurrence in several studies. In addition, anecdotal responses to EGFR inhibitors in locally advanced and recurrent tumors have been reported. The goal of this study is to evaluate EGFR expression in vulvar SCCs in correlation with HPV status of the tumor.

Design: Forty-three cases of vulvar SCC were selected. The tumors were classified as HPV-related and HPV-unrelated based on expression of p16 in all cases. EGFR expression was assessed by immunohistochemistry (IHC) in all cases and by in-situ hybridization (ISH) for EGFR mRNA by RNAscope (Advanced Cell Diagnostics) in selected 20 cases. Relatively high expression levels were observed in most of the tumors and in adjacent normal squamous epithelium in some cases. Tumors with membranous staining of higher intensity than that in the adjacent normal skin were considered positive for EGFR overexpression. The remaining cases with equal or lesser staining intensity in the tumor relative to adjacent normal were considered negative for analysis purposes. A similar approach was used for scoring ISH results. Statistical analysis was performed using R software (version 3.3.1).

Results: The study included 20 HPV-related SCCs (p16 +) and 23 HPV-unrelated tumors. Eighteen cases $(41.9 \%)$ were positive by IHC, of which $15(83 \%)$ were HPV unrelated. Twelve case (of 20,60\%) were positive by ISH, of which 9 (75\%) were HPV-unrelated. Significant correlation was observed between IHC and ISH $(\mathrm{p}<0.01)$. HPV- unrelated tumors were significantly associated with positive EGFR expression ( $\mathrm{p}$ $<0.01$ for IHC and $\mathrm{p}=0.02$ for ISH) compared to HPV-related carcinomas.

Conclusions: EGFR overexpression by IHC or ISH can be detected in a significant number of vulvar SCCs and is more common in HPV-unrelated type. Additional studies are warranted to systematically evaluate efficacy of EGFR-targeting therapy and to determine whether EGFR overexpression is predictive of therapeutic response HPV and EGFR testing may become an important modality in personalized approach to vulvar cancer care.
1195 Comparison of miRNA and mRNA Expression Profiles in Serous Carcinoma, Clear Cell Carcinoma and Carcinosarcoma of the Endometrium Sharon X Liang, Victoria Fort, Human Khalili, Ilya Korsunskiy, Andrew Shih, Veena S John, Annette Lee. Northwell Health System, Lake Success, NY; Hofstra Northwell School of Medicine, Manhasset, NY; Feinstein Institute for Medical Research, Manhasset, NY; Northwell Health System, Manhasset, NY.

Background: The Cancer Genome Atlas (TCGA) has identified four distinct molecular types of endometrial carcinoma (EMC) among a cohort of endometrioid carcinoma (EEC), serous carcinoma (SC) or mixed type carcinoma. Yet the molecular features of less common types of high grade EMC such as clear cell carcinoma (CC) and carcinosarcoma (CS) were not available in the TCGA data. The purpose of this study was to compare expression profiles of miRNAs and their target genes in SC, CC and CS. Design: EMC tissue was obtained from FFPE blocks from patients who underwent hysterectomy and surgical staging procedure at Northwell Health System. Total RNA was sequenced using the TruSeq ${ }^{\circledR}$ RNA Access Library Prep Kit (Illumina, San Diego, CA, USA) according to the manufacturer's protocol. Additionally, the expression of 754 miRNAs was measured using QuantStudio $12 \mathrm{k}$ flex instrument to generate miRNA profiles of these tumors. Both differential expression and integrated pathway analysis using mRNA and miRNA data was conducted. The significance was set at unadjusted $p$ value $<0.05$.

Results: A total of 14 EMC samples (7 SC, 4 CC and 3 CS) had sufficient RNA and were included in the study. Differential miRNA expression was noted between SC and CC, SC and CS. Differential mRNA transcription profile was also identified. SC had upregulated gene expression for ECM receptors, cell cycle markers and adhesion. CC and CS had upregulated gene expression in Jak-STAT, T-cell receptor and chemokine signaling pathways.

Conclusions: $\mathrm{SC}, \mathrm{CC}$ and $\mathrm{CS}$ demonstrated different tumor expression profiling using both miRNA and mRNA, suggesting that each of these high grade tumors may have distinct underlying molecular signatures.

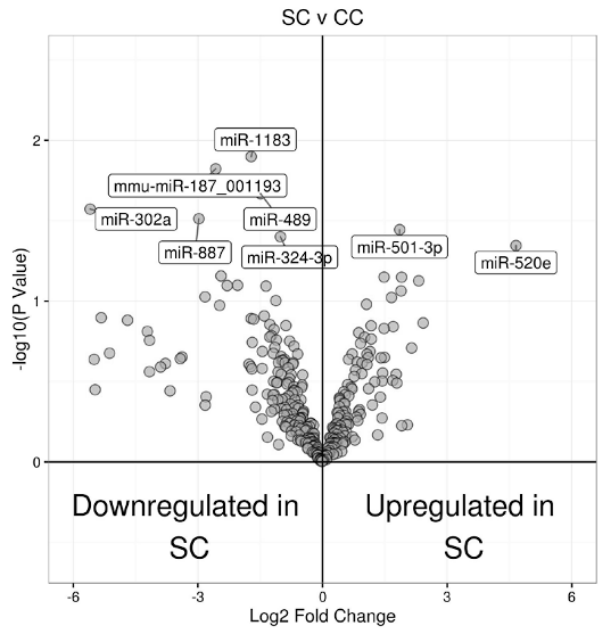

Significant At

- Raw P value

○ Not

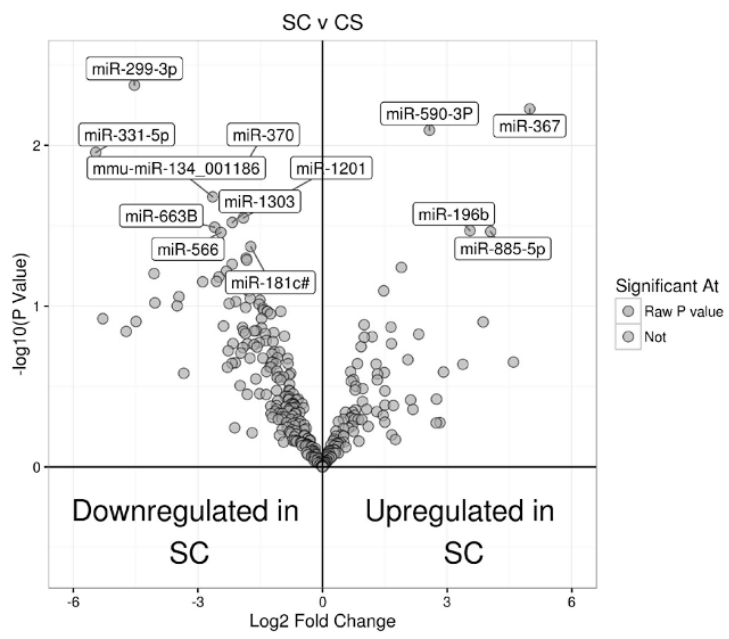

1196 Clinical Utility of Next Generation Sequencing (NGS) Panel for Recurrent/Advanced Stage Gynecological Malignancies

Diana Lim, Xiao-Wen Lee, Xue-Qing Koh, Brendan Pang, Richie Soong, David SP Tan. National University Health System, Singapore, Singapore; Centre for Translation Research and Diagnostics, Cancer Science Institute, Singapore, Singapore; National University Cancer Institute, Singapore, Singapore.

Background: Patients with recurrent/advanced stage gynecological malignancies have limited therapeutic options and often, dismal survival. We launched the "Integrated Molecular Analysis of Cancer in Gynaecologic Oncology (IMAC-GO)" program, 
one of the first precision oncology protocols in Asia, which utilizes NGS to identify potential actionable targets in this cohort, facilitating their enrolment into biomarkerenriched clinical trials.

Design: NGS utilizing a panel surveying hotspot regions of 50 cancer genes was performed using DNA extracted from formalin fixed paraffin embedded tumor tissue. Results were discussed at a molecular tumor board and incorporated for therapeutic decision-making.

Results: 81 patients were enrolled into the program from June 2014 to July 2016 NGS was feasible in 77 tumors $(95 \%)$ with reportable mutations identified in 65 patients ( 9 cervical, 16 endometrial and 40 ovarian carcinomas). In cervical cancers, $7 / 50$ (14\%) genes were mutated, most commonly involving PIK3CA (56\%; 3 SCC, 1 small cell carcinoma, 1 adenocarcinoma). One SCC showed concurrent PIK3CA and $K D R$ mutations while another had $F B X W 7$ R505G and $N R A S$ Q61K mutations. The most common mutations in endometrial tumors involved PTEN (50\%), CTNNB (48\%), PIK3CA (38\%), KRAS (25\%) and TP53 (13\%). At least 1 actionable mutation was present in $13 / 16(81 \%)$ cases, including $A T M$ and $A T K /$ mutations. Endometrial tumours with mixed endometrioid and serous phenotype exhibited similar frequency of actionable mutations as endometrioid carcinomas. $19 / 50(38 \%)$ genes were mutated in ovarian cancers. Table 1 shows the spectrum of commonly mutated genes in these tumors. One patient with synchronous endometrial and ovarian endometrioid carcinomas showed similar APC and PTEN mutations in both tumors.

\begin{tabular}{|l|l|l|l|l|}
\hline $\begin{array}{l}\text { Gene } \\
\text { Mutated }\end{array}$ & $\begin{array}{l}\text { High-grade serous } \\
\text { carcinoma }(\mathrm{n}=19)\end{array}$ & $\begin{array}{l}\text { Clear cell } \\
\text { carcinoma }(\mathrm{n}=13)\end{array}$ & $\begin{array}{l}\text { Carcinosarcoma } \\
(\mathrm{n}=6)\end{array}$ & $\begin{array}{l}\text { Mucinous } \\
\text { carcinoma }(\mathrm{n}=1)\end{array}$ \\
\hline TP53 & $89 \%$ & $23 \%$ & $33 \%$ & $100 \%$ \\
\hline PIK3CA & & $38 \%$ & $83 \%$ & \\
\hline KDR & & $23 \%$ & & \\
\hline KRAS & & $15 \%$ & $17 \%$ & \\
\hline FGFR2 & $5 \%$ & $8 \%$ & $17 \%$ & \\
\hline NRAS & & & $17 \%$ & \\
\hline PTEN & & & $17 \%$ & \\
\hline
\end{tabular}

Conclusions: A multi-marker molecular screening approaching using NGS in patient with recurrent/advanced stage gynecological malignancies allows for the discovery of potential actionable targets, which may have important therapeutic implications.

1197 Major Vault Protein Immunohistochemistry Is a Useful Adjunct in Distinguishing Leiomyosarcoma from Leiomyoma and Leiomyoma with Bizarre Nuclei

Nicholas Lintel, Scott A Koepsell, Jesse Cox, Stephen A Luebker, Subodh M Lele. University of Nebraska Medical Center, Omaha, NE; Nebraska Medicine, Omaha, NE. Background: Morphologically, distinguishing between leiomyoma (LM) and leiomyosarcoma (LMS) is not always straightforward, especially with benign variants such as bizarre leiomyoma (BLM). To identify potential markers of malignancy in uterine smooth muscle tumors, proteomic studies were performed followed by assessment of protein expression by immunohistochemistry.

Design: Archival formalin-fixed paraffin-embedded (FFPE) tissues from tumors ( $n=23$ ) diagnosed as LM, BLM and LMS (using published criteria) were selected for the study. Sequential window acquisition of all theoretical fragment ion spectra mass spectrometry (SWATH-MS) was applied to pooled samples of FFPE LM and LMS tumor tissue respectively in order to assay the relative protein quantities and look for expression patterns to differentiate the two tumor types. A total of 592 proteins were quantified in all samples, and 10 proteins were differentially expressed between LM and LMS. Select proteins were chosen for evaluation by immunohistochemistry (IHC) based on antibody availability and biologic relevance in the literature. IHC was performed on a tissue microarray and intensity was evaluated using imaging software.

Results: Major vault protein (MVP) and catechol O-methyltransferase (COMT) had 3.05 and 13.94 times higher expression in LMS relative to LM by SWATH-MS respectively. By IHC, MVP (clone 1014, Santa Cruz Biotechnology, Dallas TX) was found to be $50 \%$ sensitive and $100 \%$ specific when comparing LMS to LM. COMT (clone FL-271, Santa Cruz Biotechnology, Dallas TX) had a sensitivity of $38 \%$ and a specificity of $88 \%$. 6/7 BLM had expression of MVP similar to LM.

Conclusions: Immunohistochemical staining for MVP is a useful adjunct in distinguishing LMS from LM and BLM in difficult cases.

1198 Using P16 Immunohistochemistry to Classify CIN 2 Lesions: Correlation of Ambiguous Staining Patterns with HPV Subtypes and Clinical Outcome

Yuxin Liu, Mahfood Alqatari, Fei Ye, David Zhang, Tamara Kalir. Mount Sinai Health System, Icahn School of Medicine at Mount Sinai, New York, NY.

Background: In 2012, Lower Anogenital Squamous Terminology (LAST) project recommended using p16 immunohistochemistry (IHC) to classify Cervical Intraepithelial Neoplasia 2 (CIN 2) into the new two-tiered system: LSIL/HSIL. Beyond positive/negative results, varied p16 staining intensity and distribution pose interpretive challenges. We studied 183 CIN 2 focusing on 3 ambiguous p16 staining patterns: (1) strong/basal, (2) strong/confined, and (3) weak/diffuse staining. We set out to determine their association with specific HPV subtypes as well as positive predictive value (PPV) for HSIL.

Design: P16 IHC patterns were designated as: (A, B) strong/diffuse (block-positive in LAST terms); (C, D) weak/confined (negative in LAST); (E, F) strong but basal; $(\mathrm{G}, \mathrm{H})$ strong but confined; and (I, J) weak but diffuse. Biopsies with ambiguous p16 results were tested for HPV by PCR. Patients were followed for 12 mons.

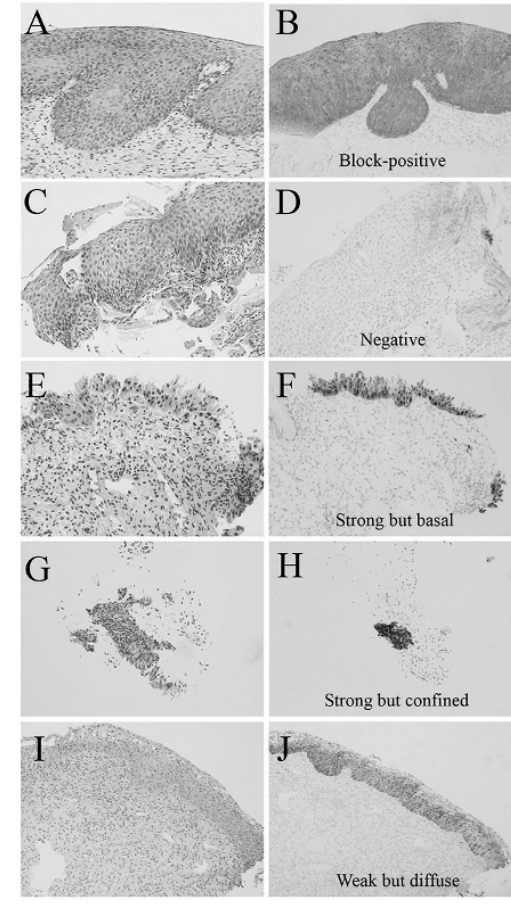

Results: Among 183 CIN 2 lesions, p16 IHC revealed positive ( $n=35,19 \%)$, negative $(\mathrm{n}=98,54 \%)$, and ambiguous patterns $(\mathrm{n}=50,27 \%)$ including 18 strong/basal, $15 \mathrm{strong} /$ confined, and 17 weak/diffuse staining. Table 1 shows correlation of HPV types with p16 results. 10 patients $(20 \%)$ with ambiguous patterns later revealed HSIL, including 9 with strong/confined and 1 with weak/diffuse staining. 40 patients $(80 \%)$ revealed LSIL, ASCUS, or negative for 12 mons.

\begin{tabular}{|c|c|c|c|c|c|}
\hline & & & \multicolumn{3}{|c|}{ Ambiguous p16 Patterns } \\
\hline $\begin{array}{c}\text { HPV } \\
\text { Genotype }\end{array}$ & $\begin{array}{c}\text { Negative } \\
(\mathrm{n}=5)\end{array}$ & $\begin{array}{c}\text { Positive } \\
(\mathrm{n}=5)\end{array}$ & $\begin{array}{c}\text { Strong/ } \\
\text { basal } \\
(\mathrm{n}=18)\end{array}$ & Strong/confined(n=15) & $\begin{array}{c}\text { Weak/diffuse } \\
(\mathrm{n}=17)\end{array}$ \\
\hline Negative & $5(100 \%)$ & - & $13(72 \%)$ & $11(73 \%)$ & $11(65 \%)$ \\
\hline Positive & - & $5(100 \%)$ & $5(28 \%)$ & $4(27 \%)$ & $6(35 \%)$ \\
\hline & & $16(\mathrm{x} 2)$ & $\begin{array}{c}16(\mathrm{x} 2) \\
59\end{array}$ & 16 & $16(\mathrm{x} 2)$ \\
HPV types & - & $18(\mathrm{x} 3)$ & 56 & 58 & $18(\mathrm{x} 2)$ \\
& & & 66 & $662)$ \\
\hline
\end{tabular}

Conclusions: In our series, 1/3 CIN 2 lesions presented ambiguous p16 IHC results. Such lesions are likely to be negative for HR-HPV or positive for non-16/18 types. It is useful to perform HPV genotyping for classification of challenging cases. Because of their low PPV (20\%) for HSIL, we recommend interpreting ambiguous p16 results with caution. In the absence of unequivocal morphologic features of HSIL, such lesions should likely be classified LSIL.

1199 Neoadjuvant Chemotherapy (NAC) in “Ovarian” High Grade Serous Carcinoma (HGSC): Pathological Response and Morphological Characterization

Francesca Locatelli, Antonio De Leo, Barbara Corti, Nunzio CM Salfi, Anna Myriam Perrone, Claudio Zamagni, Pierandrea De Iaco, Donatella DS Santini. St.OrsolaMalpighi University Hospital, Bologna, Italy.

Background: We evaluated the prognostic significance and the interobserver reproducibility (IR) of both the three-tier chemotherapy response score (CRS) validated by Böhm for the pathological response to NAC in tubo-ovarian HGSC and the morphologic pattern "Solid, pseudoEndometrioid and Transitional (SET)" proposed by Soslow as opposed to "Classic" papillary histology.

Design: The CRS was applied to omental sections of 121 patients (collected between 2001 and 2015) with stage IIIC-IV HGSC, treated with NAC and interval debulking surgery.

Diagnostic biopsy and/or adnexal and omental sections of the same series were also classified in SET and Classic based on any presence of SET-like features in at least one site.

Omental sections were independently scored for CRS and morphologic pattern by 3 pathologists and a resident. Fleiss' kappa statistics (Fleiss' K) and Kendall's coefficient of concordance (KCC) were calculated for levels of agreement. Survival functions and analyses were estimated by using the Kaplan-Meier method, the log-rank test and the Cox proportional hazards regression, adjusting for age, stage, and debulking status. For contingency tables, odds ratios (OR) and Fisher's exact test were calculated.

Results: The IR of the CRS showed high agreement (absolute agreement $=60 \%$; Fleiss' $\mathrm{K}=0.68 ; \mathrm{KCC}=0.88$ ).

The IR of the morphologic pattern revealed a moderate agreement (absolute agreement $=50 \%$; Fleiss' $\mathrm{K}=0.42 ; \mathrm{KCC}=0.53$ ). 
The CRS showed prognostic significance for progression free survival (PFS) in univariate analyses: CRS1 and CRS2 v. CRS3: median, 12 v. 19 months; Relative Risk $=0.52 ; \mathrm{p}<0.01$. In overall survival (OS) it was also significant in multivariate analyses: CRS1 and CRS2 v. CRS3: median, 28 v. 130 months; Hazard Ratio $=0.32$; $95 \%$ Confidential Interval $(\mathrm{CI})=0.12$ to $0.85 ; \mathrm{p}=0.02$.

The SET v. Classic categories revealed a nonsignificant trend for PFS and OS.

Conclusions: The CRS is easy to use, cost-free, highly reproducible and has independent prognostic significance. It identifies a set of patients with higher risk of early chemoresistant recurrence (i.e. $\mathrm{CRS} 1$ and $\mathrm{CRS} 2$ v. $\mathrm{CRS} 3$ : $\mathrm{OR}=11.1 ; 95 \% \mathrm{CI}=1,7$ to 99,2 $\mathrm{p}<0.01)$ and gives additional information to debulking status $(\mathrm{OR}=0.05 ; 95 \% \mathrm{CI}=0.009$ to $0.18 ; \mathrm{p}<0.001$ ), with implications on care. Inclusion of CRS into pathology report is justified. Morphology, evaluated as such, is not prognostic.

1200 Latent and Resolving HPV Infective Patterns in Cervical Squamous Epithelium Demonstrated by High Risk HPV mRNA Chromogenic In Situ Hybridization

Sophia Ma, Amy Ziober, Renee Frank, Kumarasen Cooper. Hospital of the University of Pennsylvania, Philadelphia, PA.

Background: A high risk (HR) HPV RNA chromogenic in situ hybridization (CISH) have been described with signaling patterns as follows: upper $1 / 3$ epithelial layer diffuse nuclear staining as a HPV productive phase infection or a low grade squamous intraepithelial (LSIL) lesion (CIN 1), strong punctate signaling throughout the epithelium as a HPV transformative infection or a high grade squamous intraepithelial (HSIL) lesion (CIN 2), and a combined transformative and productive infective pattern (CIN 2) (PLoS ONE. 2014. 9(3):e91142). This study explores the role of RNA CISH in identifying latent and resolving HPV associated lesions in cervical epithelium.

Design: 52 formalin fixed paraffin embedded cervical biopsies were selected from patients diagnosed with LSIL (CIN 1), HSIL (CIN 2) and HSIL (CIN 3) with sufficient tissue material. Immunohistochemical staining for $\mathrm{p} 16$ was performed using a heatactivated, peroxidase/diaminobenzidine-complex method, and RNA CISH targeted HR HPV branched DNA/RNA using RNA Scope (Advanced Cell Diagnostics). RNA $\mathrm{CISH}$ expression patterns were defined as previously described. Comparisons were made between the RNA CISH and $\mathrm{p} 16$ expression patterns.

Results: Of the 52 patients, 30\% (16) had HPV transformative infection; 6\% (3) had HPV productive phase infection; $20 \%(10)$ had an admixture of the two; and $21 \%(11)$ were negative. These patterns are concordant with $\mathrm{p} 16$ immunoexpression patterns as described by Darragh et al (JLow Genit Tract Dis. 2013 Jul;17(3):368.). The remaining $23 \%$ (12) patients had LSIL morphology with p16 expression was either negative or had only cytoplasmic staining. These cases demonstrated a scattered, finely granular signal pattern located in the lower $1 / 3$ of the epithelium in RNA CISH. $92 \%(11 / 12)$ of these patients had a positive HR cobas HPV DNA test on the most recent PAP test. Only $9 \%$ of these latter group showed relapse of squamous lesions on subsequent pap studies. In contrast, $24 \%$ of prior patent population had recurrence of squamous lesions on subsequent pap test $(\mathrm{p}<0.05)$.

Conclusions: The results of the data demonstrate a putative identification of latent/ resolving HPV infection on RNA CISH corresponding to a reactive or negative p16 immunoexpression in patients with positive HR HPV DNA by PAP test. This study expands the utility of HPV RNA CISH to better categorize HPV infection in cervical biopsies to include latent or regressing lesions.

1201 Transitional Cell Carcinomas (TCC) and Non-TCC High Grades Serous Carcinomas (HGSC) of the Ovary Share Identical Immunostaining Profiles Using Established HGSC Markers

Jamie Magrill, Stefan Kommoss, David G Huntsman, C Blake Gilks, Dawn R Cochrane, Aline Talhouk, Robert Soslow, Anthony N Karnezis, Basile Tessier-Cloutier, Christine Chow, Angela S Cheng, Andreas du Bois, Jacobus Pfisterer, Friedrich Kommoss. BC Cancer Agency, Vancouver, BC, Canada; Vancouver General Hospital, Vancouver, BC, Canada; Tübingen University Hospital, Tübingen, Germany; Memorial Sloan Kettering Cancer Center, New York, NY; Kliniken Essen Mitte, Essen, Germany; Gynecologic Oncology Center, Kiel, Germany.

Background: Transitional cell carcinoma of the ovary (TCCO) is grouped with high grade serous carcinoma (HGSC) in the current WHO classification. TCCO is associated with BRCA mutations and a better prognosis compared to HGSC. Previous papers on TCCO have studied limited numbers of samples. No marker reflecting the biological difference between TCCO and HGSC is known. Therefore, we collected a large cohort of TCCO to determine whether TCCO and HGSC could be distinguished by immunohistochemistry (IHC)

Design: A tissue microarray (TMA) was built from 89 TCCO and a small control cohort of 16 conventional HGSC. IHC was performed, scored and statistically analyzed for routine markers of HGSC and urothelial tumors: PAX8, WT1, p53, p16, ER, p63 and GATA3.

Results: The IHC profile of TCCO was very similar to HGSC. The differences in the proportions for each of the markers between the two groups were not statistically significant ( $\mathrm{p}$-values $>0.5$ ).

\begin{tabular}{|c|c|c|c|c|c|c|c|}
\hline & $\begin{array}{c}\text { PAX8 } \\
\text { positive }\end{array}$ & $\begin{array}{c}\text { WT1 } \\
\text { positive }\end{array}$ & $\begin{array}{c}\text { p53 } \\
\text { mutant }\end{array}$ & $\begin{array}{c}\text { p16 } \\
\text { positive }\end{array}$ & $\begin{array}{c}\text { ER } \\
\text { positive }\end{array}$ & $\begin{array}{c}\text { p63 } \\
\text { negative }\end{array}$ & $\begin{array}{c}\text { GATA3 } \\
\text { negative }\end{array}$ \\
\hline \multirow{2}{*}{ TCCO } & $81 / 88$ & $79 / 88$ & $80 / 85$ & $85 / 89$ & $83 / 88$ & $86 / 87$ & $81 / 88$ \\
& $(92 \%)$ & $(90 \%)$ & $(94 \%)$ & $(96 \%)$ & $(94 \%)$ & $(99 \%)$ & $(92 \%)$ \\
\hline \multirow{2}{*}{ HGSC } & $16 / 16$ & $15 / 16$ & $13 / 14$ & $16 / 16$ & $16 / 16$ & $16 / 16$ & $14 / 16$ \\
& $(100 \%)$ & $(94 \%)$ & $(93 \%)$ & $(100 \%)$ & $(100 \%)$ & $(100 \%)$ & $(88 \%)$ \\
\hline
\end{tabular}

Conclusions: TCCO and HGSC cannot be distinguished using established immunomarkers for HGSC and urothelial tumors even when applied to this large series of TCCO. Therefore further studies using proteomic analysis will focus on differing expression of proteins that might serve as markers of TCC-like differentiation, which could help explain biological differences between TCCO and HGSCO and might identify other cases of HGSCO with a better prognosis.

1202 Differentiated Vulvar Intraepithelial Neoplasia Associated with Intraepithelial Neoplastic Spread and with Usual Vulvar Intraepithelial Neoplasia

Kien T Mai. University of Ottawa, Ottawa, ON, Canada.

Background: Differentiated vulvar intraepithelial neoplasia (dVIN) associated with intraepithelial neoplastic spread and with usual vulvar intraepithelial neoplasia (uVIN) has not been studied

Design: Consecutive cases of vulvar intraepithelial neoplasia (VIN) diagnosed between 2011-2013 were reviewed. The overlying and adjacent epithelium was categorized into Group $1:$ a) dVIN characterized by atypical keratinized cells and wide intercellular spaces creating a "cobblestone" appearance in the basal/parabasal layers; b) uVIN and c) dVIN associated with atypical neoplastic spread above the parabasal layer in the usual-like VIN pattern (u-like VIN).

Results: Two- hundred-thirty-five consecutive cases of VIN including 54 invasive squamous cell carcinoma (SCC) were identified. There were $12 \mathrm{dVIN}$ (invasive, $\mathrm{n}=8$, or $67 \%$ of cases) (group 1), $178 \mathrm{uVIN}$ (invasive, $\mathrm{n}=5$ or $3 \%$ ) (Group 2), and $45 \mathrm{u}$-like VIN with or without $\mathrm{uVIN}$ (invasive, $\mathrm{n}=41$ or $91 \%$ ) (Group 3) (Fig 1). u-like VIN surface exhibited keratinization with lack of granular layer as commonly seen in keratinizing dysplasia (KD). In addition, u-like VIN was often associated with focal dVIN with transitional changes between dVIN and u-like VIN or uVIN. P16 was immunoreactive in the overlying epithelium and in the invasive SCC in $8 \%, 69 \%$ and $40 \%$ of cases of Groups 1,2 and 3 respectively.

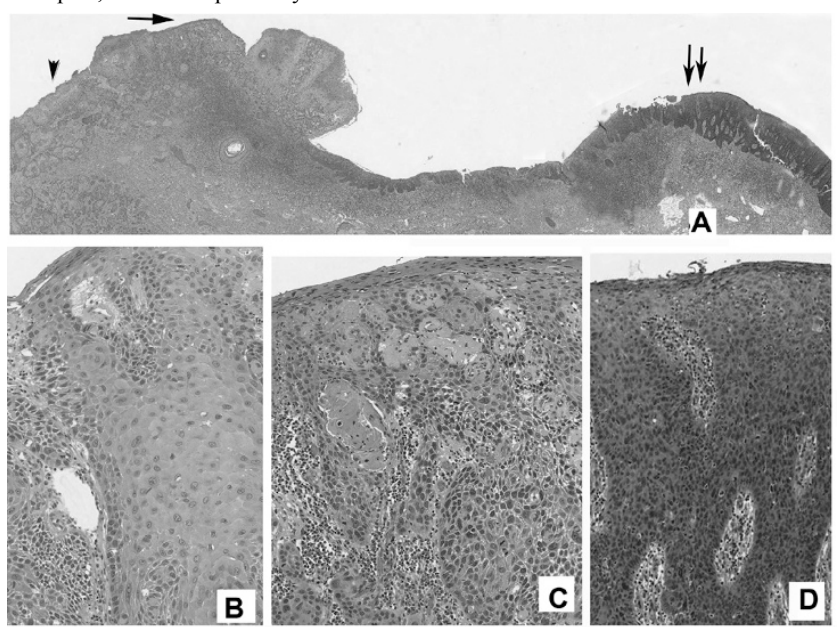

Fig 1A showing an admixture of dVIN with ICC (arrowhead and Fig B), u-like VIN (arrow and Fig 1C) and uVIN (double arrow and Fig 1D)

Conclusions: The changes in u-like VINare suggestive of intraepithelial and upward spread of neoplastic cells with early keratinizaton from the basal/parabasal layers of dSIN, as a mechanism of the development of KD in u-like VIN . As a result, KD is prone to develop ISCC due to the inherent features of dVIN in KD. Since u-like VIN is commonly associated with invasive SCC, its distinction from UVIN is of pathological and clinical significance.

1203 Low-Grade-Like High- Grade Squamous Intraepithelial Lesion of the Uterine Cervix: Histologic Characterization of Equivocal Dysplasias and Correlation with Human Papillomavirus Genotype

Ramya Masand, Barrett Lawson, Derek Danner, Matthew Anderson, Ninad M Patil. Baylor College of Medicine, Houston, TX; Ben Taub General Hospital, Houston, TX Background: In this study, we describe the histology of these low-grade-like highgrade squamous intraepithelial lesions (LLHSIL). We also correlate LLHSIL with HPV genotype.

Design: Retrospective review of all cases equivocal for LSIL vs HSIL that were sent for gynecologic pathologist review \& stained for p16 and Ki67, was performed from Jan. 2015 to Sept. 2016. The histologic features, IHC results and HPV genotype results (APTIMA ASSAY) were recorded.

Results: Out of 94 cases, $70(74.4 \%)$ were non16,non-18 HPV type, 7 (7.5\%) were HPV16+ , $10(10.6 \%)$ were HPV 18+, and $7(7.5) \%$ were high-risk HPV (HRHPV) negative. Of non-16,non-18 HPV cases, 57 HSIL \& 13 LSIL. HPV 16+ cases: 5 HSIL and 2 LSIL. HPV 18+ cases: 7 HSIL and 3 LSIL. HRHPV negative cases: 3 HSIL and 4 LSIL. The non 16/18 HPV HSIL population had a characteristic morphology that we termed LLHSIL: Cytomegaly with only mild increase in nucleo-cytoplasmic ratio, anisonucleosis, bi- and multinucleation throughout the epithelial thickness, mild -moderate nuclear atypia, increased eosinophilia and/ or glycogenation of the superficial layers giving the appearance of diffuse koilocytosis, lack of nuclear crowding and elongation especially in the basal layers seen in typical HSIL. Mitotic activity was low and limited to the lower layers. P16 showed diffuse band like staining but was less intense in superficial areas with cytoplasmic clearing giving a dark to light gradient from basal to superficial layers in some cases. Ki67 showed full thickness positivity. 

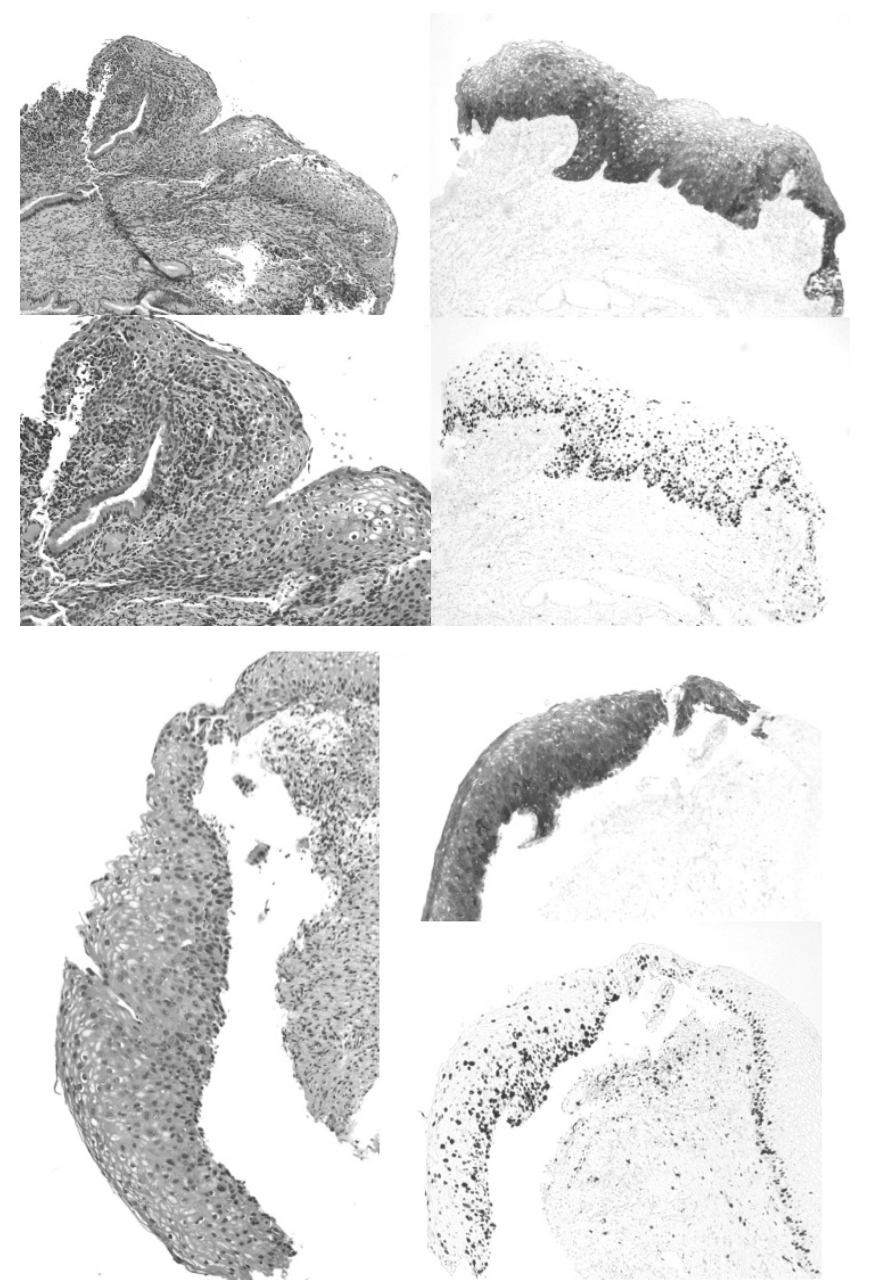

Conclusions: In this study, we describe LLHSIL morphology mimics LSIL, and typically requires IHC for proper characterization. This morphology appears to be more common with non 16/18 genotypes. Awareness of this variant morphology and use of IHC will help classify these LLHSIL lesions accurately.

1204 Recurrence of Low-Grade Low-Stage Endometrial Cancer Is Associated with Broad Copy Number Gain and Higher Rates of PIK3CA Mutation

Cathleen E Matrai, Sudeshna Chatterjee-Paer, Andrea Sboner, Juan Miguel Mosquera Kevin Holcomb, Divya Gupta, Mark A Rubin, Lora Ellenson. Weill Cornell Medicine, New York, NY; Englander Institute for Precision Medicine, New York, NY; Institute for Computational Biomedicine, New York, NY.

Background: Low-grade, low-stage endometrioid carcinomas (LGLS EC) demonstrate 5 -year survival rates up to $95 \%$. However, a small subset recurs and no prognostic markers are currently available. The goal of our study was to identify genomic alterations that may predict recurrence by comparing the molecular profiles of LGLS EC with recurrence to LGLS EC without recurrence.

Design: We studied four cases of LGLS EC with recurrence and six age and histology matched controls. Tumor morphology was reviewed by two pathologists with expertise in gynecologic pathology (LHE, CM). Paired primary tumor and germline DNA of each case underwent whole exome sequencing. A total of 21,522 genes were analyzed using a comprehensive computational pipeline to detect point mutations, indels, and copy number alterations. Mutations were categorized as: 1) somatic alterations in clinically relevant genes, 2) other alterations in cancer genes, and 3) alterations of unknown significance. P-values were calculated using Fisher's exact probability test.

Results: Our data showed 3 of 4 LGLS EC with recurrence demonstrated broad copy number gains $(B C G)$ in multiple genes that were absent from controls $(p=0.03)$. The case with recurrence that did not show BCG showed multiple focal copy number gains and was PIK3CA mutated. Three of four recurrent cases had PIK3CA mutations while mutation was only detected in one of six cases without recurrence. However, this trend did not reach statistical significance.

Conclusions: Although LGLS EC has a $95 \%$ five year survival, $5 \%$ of women die of the disease. The underlying cause as to why certain tumors recur despite a lack of histologic or clinical evidence portending aggressive behavior has not yet been elucidated. Our data support that a subset of LGLS EC may be genetically predisposed to recurrence. The TCGA has noted that the majority of uterine serous tumors, $25 \%$ of high-grade endometrioid tumors, and a small percentage of lower grade (1 and 2) endometrioid tumors $(<5 \%)$ show extensive copy number alterations, a finding similar to that seen in our LGLS cases with recurrence. Further investigation into the significance of these findings in larger cohorts would be warranted to identify patients who may be at higher risk of recurrence and need alternative treatment strategies.
1205 Usual Type Endocervical Adenocarcinoma/AIS Incidence and Distribution of High-Risk HPV Genotypes Between 2007-2010 and 20112015

Whitney A McCarthy, Yiang Hui, Berlly L Diaz-Gomez, Joyce Ou, Cherie Paquette, C James Sung, W Dwayne Lawrence, M Ruhul Quddus. Women and Infants Hospital of Rhode Island/Warren Alpert Medical School of Brown University, Providence, RI; Clinica Universitaria Colombia--Grupo de Investigacion INPAC, Bogota DC, Cundinamarca, Colombia.

Background: Usual type endocervical adenocarcinoma (ECA) and its precursor lesion (AIS) occur in young to middle-aged women and are associated with high-risk HPV (HRHPV), most commonly 16/18. ECA/AIS incidence apparently has increased possibly as a result of increased identification by pathologists. Since $16 / 18$ have been included in vaccines, 16/18-driven carcinomas should decrease. We have also anecdotally observed that the average age of patients with ECA/AIS at our institution has decreased. We aim to compare average age at diagnosis between two time periods (2007-2010 and 20112015), and to characterize predominant HR-HPV strains.

Design: ECA/AIS cases from 2007-15 were reviewed. DNA was extracted from FFPE tissue with the QIAam DNA FFPE Kit, tested using GenomeMe ${ }^{\mathrm{TM}}$ 's GeneNav ${ }^{\mathrm{TM}} \mathrm{HPV}$ One qPCR Kit (GenomeMe, Richmond, BC, Canada) for detection of HPV 16 \& 18; non-16/18 samples were typed by GenomeMe ${ }^{\mathrm{TM}}$ 's GeneNav ${ }^{\mathrm{TM}}$ HPV Genotyping qPCR Kit. Mean and median age at diagnosis was calculated, and a p-value comparing means between the two time periods was calculated using a Mann-Whitney U test. HR-HPV frequency from 2007-10 to 2011-15 was compared using a Chi-squared test.

Results: Mean/median age at diagnosis for the two time periods are included in Table $1(\mathrm{p}=0.459)$. HPV type frequencies are shown in Figure $1(\mathrm{p}=0.444)$.

\begin{tabular}{|l|l|l|}
\hline & $2007-10$ & $2011-15$ \\
\hline Mean age (yrs) & 48.2 & 46.2 \\
\hline Median age (yrs) & 47 & 42 \\
\hline
\end{tabular}

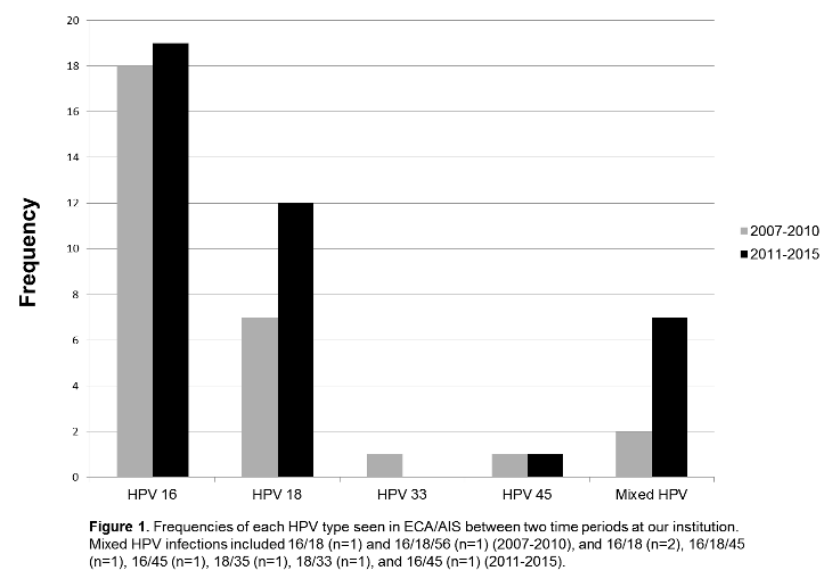

Conclusions: Multi-strain HPV infection was 3 times more common in the 2011-15 group. No significant difference in age at diagnosis was observed between the two time periods. HR-HPV types 16/18 remain the most prevalent types identified in ECA/ AIS; no significant difference was seen in the HR-HPV frequencies between 2007-10 \& 2011-15. The increase in multi-strain HR-HPV infection may indicate that it becoming more common, necessitating the widespread development/use of vaccines with broad coverage for HR-HPV strains. Further study will be needed to evaluate trends in HR-HPV and ECA/AIS.

1206 Comparison of p63 and p40 Immunohistochemical Stains to Distinguish Epithelioid Trophoblastic Tumor from Other Trophoblastic Lesions

Whitney A McCarthy, Cherie Paquette, W Dwayne Lawrence. Women and Infants Hospital of Rhode Island/Warren Alpert Medical School of Brown University, Providence, RI.

Background: Trophoblastic neoplasms such as placental site trophoblastic tumor (PSTT) and epithelioid trophoblastic tumor (ETT) are very rare, diagnostically challenging, and potentially aggressive malignancies. The importance of distinguishing these 2 tumors from one another, and from benign trophoblastic proliferations such as placental site nodule (PSN) and exaggerated implantation site (EIS), cannot be overemphasized. P63 has been shown to be a helpful marker in distinguishing the chorionic-type trophoblasts in ETT and PSN from implantation site trophoblast in PSTT and implantation site trophoblast/syncytiotrophoblast in EIS. Recently, a truncated isoform of p63, p40, has shown improved specificity compared to p63 in tumors such as pulmonary squamous cell carcinoma, and some laboratories have replaced p63 with p40. In this study, we aim to compare p63 and p40 immunohistochemistry in ETT, PSTT, PSN, and EIS to determine if p40 performs similarly to p63.

Design: Cases of ETT, PSTT, PSN, and EIS from 1995-2015 were reviewed, and one FFPE tissue block was stained with both p63 (BC4A4) and p40 (polyclonal) immunohistochemical stains (GenPath Diagnostics, Elmwood Park, NJ). Using ImageJ software (NIH, Bethesda, MD) as a cell counter to calculate the number of staining nuclei, 5 HPF (400x, Olympus BX40 microscope) were assessed per case, and averages were calculated for each group. Groups were then compared using a Mann-Whitney U test. 
Results: No significant difference was seen between the percentage of nuclei stained by p63 versus p40 in PSN and ETT (Table 1). All PSTT and EIS were uniformly negative for $\mathrm{p} 63$ and $\mathrm{p} 40$.

\begin{tabular}{|l|l|l|l|}
\hline & $\%$ nuclei p63 $+($ avg $)$ & $\%$ nuclei p40 $+($ avg $)$ & P value \\
\hline PSN $(n=6)$ & $72 \%$ & $69 \%$ & $\mathrm{p}=0.456$ \\
\hline ETT $(n=3)$ & $89 \%$ & $89 \%$ & $\mathrm{p}=0.316$ \\
\hline PSTT $(n=4)$ & $0 \%$ & $0 \%$ & \\
\hline EIS $(n=7)$ & $0 \%$ & $0 \%$ & \\
\hline
\end{tabular}

Conclusions: This study of rare trophoblastic tumors demonstrates equivalent immunoreactivity for $\mathrm{p} 63$ and $\mathrm{p} 40$, suggesting that $\mathrm{p} 40$ may be used in a manner similar to $\mathrm{p} 63$ in the diagnosis of ETT and PSN. A positive $\mathrm{p} 40$ excludes the common mimics (PSTT and EIS). For laboratories supplanting p63 with p40, p40 may be considered useful in an algorithmic approach to the diagnosis of trophoblastic neoplasms, with a larger study needed to confirm our findings.

1207 Differentiated Squamous Intraepithelial Lesion (dSIL)-Like Changes in the Epidermis Overlying Anogenital Melanocytic nevi. A Diagnostic Pitfall

Kvetoslava Michalova, Dmitry V Kazakov, Michael Michal, Ladislav Hadravsky, Denisa Kacerovska, Marketa Miesbauerova, Boris Rychly, Michal Michal. Charles University, Medical Faculty and Charles University Hospital, Plzen, Czech Republic; Charles University, Third Medical Faculty and Charles University Hospital KralovskeVinohrady, Prague, Czech Republic; Cytopathos, Bratislava, Slovakia (Slovak Republic).

Background: Differentiated squamous intraepithelial lesion (dSIL) is a premalignant lesion analogous in the whole anogenital region. We describe a peculiar change in the basal cell layer of the epidermis overlying anogenital melanocytic nevi that imitates dSIL. The aim of this study is to familiarize pathologists with this pitfall to avoid its overdiagnosis as dysplasia. We tried to explore the biological characteristics of dSILlike changes and to focus on the differential diagnostic aspects.

Design: Seventy anogenital nevi were retrieved, stained with hematoxylin-eosin and reviewed. Cases in which the epidermis overlying nevi featured atypical appearing basal keratinocytes in otherwise differentiated epithelium, variable degrees of acanthosis and parakeratosis, were selected for additional investigation.

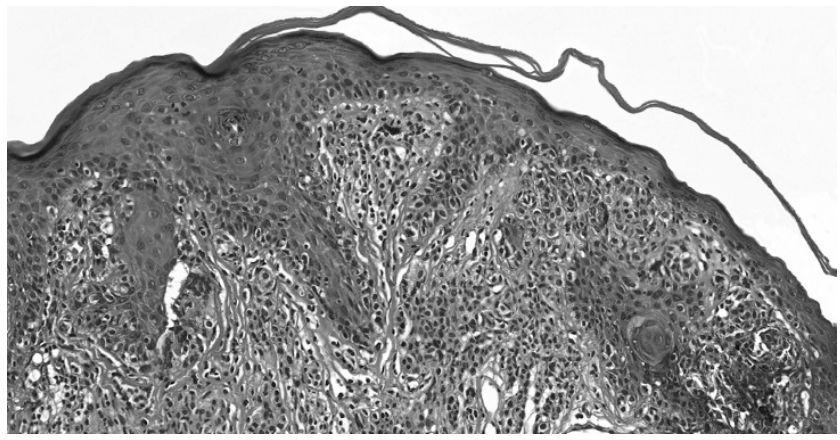

Results: Thirty cases meeting the above described criteria were identified. Patients were 8 males and 22 females, with age ranging from 4 to 68 years. Follow-up data were available for 28 patients and to date, no signs of epithelial malignancy have been recorded. Immunohistochemically, the epidermis overlying nevi showed insignificant positivity for p53 in all tested cases. Melanocytic markers (S-100 protein, SOX10, Melan A) and cytokeratin AE1/3 labeled melanocytes and keratinocytes, respectively, enabling their distinction.

Conclusions: dSIL-like changes seem to occur relatively often in the epidermis overlying anogenital melanocytic nevi. Since morphologically they are virtually identical to the "true" dSIL, their distinction largely depends on p53 expression in basal keratinocytes. The normal p53 expression in dSIL-like changes and diffuse nuclear/ p53-null immunostaining in the "true" dSIL serves as an essential differential diagnostic tool. dSIL-like alterations seem to have no malignant potential.

1208 CK7 Immunohistochemistry as a Predictor of CIN1 Progression: A Retrospective Study of Patients from the Quadrivalent HPV Vaccine Trials

Anne Mills, Cherie Paquette, Tatjana Terzic, Philip E Castle, Mark H Stoler. University of Virginia, Charlottesville, VA; Albert Einstein College of Medicine, Bronx, NY. Background: Although most women with cervical LSIL (CIN1) clear their disease without intervention, $\sim 10 \%$ develop HSIL (CIN2-3). There has been interest in the use of biomarkers to identify the subset of CIN1 most likely to progress. CIN2-3 potentially arises from a distinct population of cells at the squamocolumnar junction (SCJ), therefore the use of immunohistochemical (IHC) stains that characterize the SCJ phenotype, including CK7, has been proposed for CIN1 risk stratification. However, the prognostic power of CK7 in CIN1 biopsies has not been well established.

Design: We conducted a retrospective study of CK7 IHC on adjudicated CIN1 tissue from patients in the placebo arm of the quadrivalent human papillomavirus (HPV) vaccine trials, women with optimized disease ascertainment over 2 years follow-up. Tissue sections were stained with CK7 IHC and the lesional epithelium was scored as negative, patchy, gradation ("top-down"), or full-thickness pattern. Results were assessed for prediction of future diagnosis of CIN2-3/AIS, with analysis including p16 IHC, antecedent cytology, and HPV16 status.
Results: 517 patients with CIN1 biopsies and complete data were identified, $12 \%$ of which showed CIN2+ progression. Full-thickness CK7 staining showed the highest correlation with CIN2+ progression [Odds Radio (OR) 2.8, $p=0.021$ ] relative to the other risk factors (HPV16: OR 2.0, $p=0.035$; antecedent high-grade cytology: OR 2.2, $p=0.028$; 16 IHC: OR $1.5, p=0.16$ ). The prognostic significance of full-thickness CK7 was amplified when progression was defined as CIN3/AIS (OR 5.7, $p=0.018$ ); however, this was based on only 6 cases and the $95 \%$ confidence interval was broad (1.4-24). Inclusion of the gradation pattern resulted in a less robust association with progression (CIN2+: OR 2.0, $p=0.028$; CIN3+: OR 1.3, $p=0.74)$. Interobserver variability ranged from slight to substantial and was not contingent on gynecologic pathology training experience ( $\mathrm{K}$ for negative/patchy vs. gradation/full-thickness: 0.708 ; $\mathrm{K}$ for negative/ patchy/gradation vs. full-thickness: 0.567 ).

Conclusions: These data support the theory that SCJ-derived CIN1s are precursors to a potentially more aggressive subset of cervical SILs and that CK7 staining may inform risk stratification for CIN1. Clinical utility is tempered by variability in interobserver agreement and sampling issues. These limitations argue against the routine use of CK7 IHC in prognosis andtreatment stratification for CIN1.

\section{Comparison of Automated HR-HPV E6/7 mRNA ISH Against} PCR, DNA ISH, and p16 IHC in 102 Samples of HPV-Related Neoplasia

Anne Mills, Dawn C Dirks, James Bowden, Melinda D Poulter, Mark H Stoler. University of Virginia, Charlottesville, VA.

Background: Dysregulated expression of oncogenic types of E6 \& E7 is necessary for HPV-driven carcinogenesis. An HPV E6/E7 mRNA ISH assay covering 18 common high-risk types ("HR-RISH," using RNAScope from Advanced Cell Diagnostics) has not been extensively studied in the genital tract, validated on automated technology, or compared to existent tissue-based methods of HPV detection. We compare HR-RISH to DNA PCR, p16 IHC, and a previously available HPV DNA ISH assay in HPV-related anogenital and head and neck $(\mathrm{H} \& \mathrm{~N})$ neoplasias.

Design: 102 squamous intraepithelial lesions (16 CIN 1, 25 CIN3, 3 AIN1, 12 AIN3, 9 VIN3)/invasive squamous cell carcinomas (SCC) (17 cervix, 2 anal, 18 p16+ H\&N) as well as 10 normal and 15 reactive cervix samples were collected. HR-RISH, DNA ISH, and p16 IHC were performed on whole FFPE sections. RNA ISH for 6 low-risk HPV types (LR-RISH) was also performed. RNA and DNA ISH assays used automated systems (Leica BOND RX and Ventana Benchmark respectively). HR-HPV PCR was performed on morphology-directed block punches (cobas HPV, Roche).

Results: HR-RISH was $\geq 97 \%$ sensitive for PCR+ and p16+ neoplasia, as well as morphologically defined anogenital HSIL/invasive SCC. HR-RISH was also positive in $78 \%$ of LSIL and a subset of PCR-/invalid \&p16- lesions. All HR-RISH- H\&N SCC were oral/laryngeal \& negative for PCR, suggesting etiology unrelated to HPV. All normal cervix samples and all but 1 reactive cervix samples were HR-RISH-

\begin{tabular}{|c|c|c|c|c|c|}
\hline \multicolumn{6}{|c|}{ PCR as gold standard } \\
\hline & $\begin{array}{l}\text { HR-RISH + } \\
(\mathrm{n}=94)\end{array}$ & $\begin{array}{l}\text { LR-RISH } \\
+(\mathrm{n}=5)\end{array}$ & $\begin{array}{l}\text { DNA ISH + } \\
(\mathrm{n}=95)\end{array}$ & $\begin{array}{l}\mathrm{p} 16 \mathrm{IHC}+ \\
(\mathrm{n}=89)\end{array}$ & \\
\hline $\begin{array}{l}\text { HR-HPV PCR + } \\
(\mathrm{n}=86)\end{array}$ & $98 \%$ & $3 \%$ & $97 \%$ & $93 \%$ & \\
\hline $\begin{array}{l}\text { HR-HPV PCR - } \\
(\mathrm{n}=28)\end{array}$ & $18 \%$ & $7 \%$ & $29 \%$ & $14 \%$ & \\
\hline $\begin{array}{l}\text { HR-HPV PCR } \\
\text { invalid }(n=13)\end{array}$ & $38 \%$ & $0 \%$ & $31 \%$ & $38 \%$ & \\
\hline \multicolumn{6}{|l|}{ p16 as gold standard } \\
\hline & $\begin{array}{l}\text { HR-RISH + } \\
(\mathrm{n}=94)\end{array}$ & $\begin{array}{l}\text { LR-RISH } \\
+(\mathrm{n}=5)\end{array}$ & $\begin{array}{l}\text { DNA ISH + } \\
(\mathrm{n}=95)\end{array}$ & $\begin{array}{l}\mathrm{PCR}+ \\
(\mathrm{n}=86)\end{array}$ & \\
\hline $\mathrm{p} 16+(\mathrm{n}=89)$ & $97 \%$ & $22 \%$ & $92 \%$ & $\begin{array}{l}93 * \mathrm{vs} \\
90 \% * *\end{array}$ & \\
\hline $\mathrm{p} 16-(\mathrm{n}=38)$ & $21 \%$ & $8 \%$ & $46 \%$ & $\begin{array}{l}20 * \text { vs } \\
16 \% * *\end{array}$ & \\
\hline \multicolumn{6}{|c|}{ Morphology as gold standard } \\
\hline & $\begin{array}{l}\text { HR-RISH+ } \\
(\mathrm{n}=94)\end{array}$ & $\begin{array}{l}\text { LR-RISH } \\
+(\mathrm{n}=5) \\
\end{array}$ & $\begin{array}{l}\text { DNA } \\
\mathrm{ISH}+(\mathrm{n}=95)\end{array}$ & $\begin{array}{l}\text { p16 } \\
\mathrm{IHC}+(\mathrm{n}=89)\end{array}$ & $\mathrm{PCR}+(\mathrm{n}=86)$ \\
\hline $\begin{array}{l}\text { Anogenital HSIL/ } \\
\text { SCC }(n=66)\end{array}$ & $97 \%$ & $5 \%$ & $97 \%$ & $94 \%$ & $\begin{array}{l}97 * \text { vs } \\
89 \% * *\end{array}$ \\
\hline \multirow[t]{2}{*}{$\begin{array}{l}\text { Anogenital LSIL } \\
(\mathrm{n}=18)\end{array}$} & $78 \%$ & $11 \%$ & $61 \%$ & $50 \%$ & $59 *$ vs $56 \%$ \\
\hline & $83 \%$ & $0 \%$ & $83 \%$ & N/A & $78 \% * / * *$ \\
\hline
\end{tabular}

*Excludes vs. **includes PCR invalid cases

Conclusions: HR-RISH is a robust method for the detection of HR-HPV-related neoplasia and provides insight into HPV pathobiology. Performance meets or exceeds that of existing assays in anogenital and H\&N lesions.

1210 Placental Site Nodule, Atypical Placental Site Nodule and Epithelioid Trophoblastic Tumor: Clinical-Pathologic Characterization and Comparison of Trophoblastic and Stromal Cell Populations

Jelena Mirkovic, Elizabeth Kehr, Brooke E Howitt, Christopher P Crum, Carlos ParraHerran. Brigham and Women's Hospital, Boston, MA; Sunnybrook Health Sciences Centre, Toronto, ON, Canada.

Background: Placental site nodule (PSN) is a lesion with low proliferation index and indolent course. Atypical PSN (ASN) is defined as PSN with $2 / 3+$ atypia or borderline proliferation index (8-10\%). The prevalence of ASN and its association with epithelioid trophoblastic tumor (ETT) are not fully characterized. We aim to characterize the clinical and pathologic spectrum of PSN/ASN with emphasis on comparing cell population profiles of PSN, ASN and ETT. 
Design: We retrospectively identified cases diagnosed as PSN, ASN or ETT in a 14-year period. Features assessed included cellularity, mitoses, atypia, necrosis and presence of decidualized stroma. Immunohistochemistry for AE1/AE3-MIB-1, CD146-MIB-1, p63 and cyclin E was performed. Follow-up information was retrieved, when available. Results: 28 PSN, 8 ASN and 4 ETT with available material were identified. All PSN/ ASN were microscopic and found incidentally in endometrial biopsy. Most trophoblast cells were of "immature (epithelioid) extravillous" type (p63+, CD146-), but a minor component of "mature extravillous" type trophoblast (CD146+, p63-) was seen in 39\% PSN, 25\% ASN and all ETT. Trophoblast nuclear cyclin E expression was detected in all cases. 100\% ASN, 57\% PSN and one ETT contained decidualized cells in the periphery of the lesion, which showed cytoplasmic cyclin E staining. Follow-up was available in 21 PSN and 7 ASN patients; of these, 16 (57\%) had further endometrial sampling. All were alive with no evidence of trophoblastic neoplasia.

\begin{tabular}{|l|l|l|l|}
\hline Feature & ISN & ASN & ETT \\
\hline Atypia 2+/3+ & $0 \%$ & $75 \%$ & $100 \%$ \\
\hline Mean MIB-1 index (range) & $2.5(0-7)$ & $6.7(1-10)$ & $12(8-14)$ \\
\hline Average mitoses (range) & 0 & $0.3(0-1)$ & $3.7(0-7)$ \\
\hline Necrosis & $11 \%$ & $12 \%$ & $75 \%$ \\
\hline Decidual cells & $57 \%$ & $100 \%$ & $33 \%$ \\
\hline Mean\% CD146+ cells & $11 \%$ & $6 \%$ & $30 \%$ \\
\hline Mean\% p63+ cells & $89 \%$ & $94 \%$ & $70 \%$ \\
\hline Nuclear (trophoblast) Cyclin-E & $100 \%$ & $100 \%$ & $100 \%$ \\
\hline
\end{tabular}

Conclusions: ASN comprises $22 \%$ of all PSN in our series. PSN and ASN have a mixed cell population, containing varying proportions of mature and immature extravillous trophoblast and eliciting a decidual response in the immediately adjacent stroma. A similar mixed population was observed in ETT, which merits further investigation. ASN as currently defined appears to have a good prognosis with no documented progression to ETT in our series; additional tools to identify cases at risk of malignant progression are needed. Based on our data, nuclear cyclin $\mathrm{E}$ is not helpful in distinguishing ASN from ETT.

\section{Gestational Trophoblastic Tumors Are Characterized by FOSB \\ Expression}

Jelena Mirkovic, Bradley J Quade, Jason L Hornick, Michelle S Hirsch. Brigham and Women's Hospital, Boston, MA

Background: FOSB and other members of the activating protein-1 (AP-1) family of transcription factors are important regulators of cellular proliferation, differentiation and invasion processes in the human placenta. FOSB immunohistochemistry (IHC) expression was previously demonstrated in non neoplastic extravillous trophoblast and choriocarcinoma, but has not been thoroughly evaluated in all trophoblast-related proliferations and neoplasia.

Design: FOSB IHC was performed in 10 placentas (5 immature and 5 mature), 8 implantation site nodules (ISN; including 3 atypical ISNs), 12 hydatidiform moles (5 partial, PHM; 7 complete, CHM), and 20 gestational trophoblastic tumors, including 10 choriocarcinomas (CH-CA), 6 placental site trophoblastic tumors (PSTT), and 4 epithelioid trophoblastic tumors (ETT). 160 endometrial adenocarcinomas on a TMA were also stained for comparison. Intensity (negative, mild, moderate, strong) and distribution (focal, $<50 \%$ or multifocal/diffuse, $>50 \%$ ) of nuclear immunoreactivity were evaluated.

Results: All cases of IP, PHM, and CHM exhibit multifocal, moderate to strong expression of FOSB in extravillous trophoblast and implantation site trophoblast, rare weak to multifocal strong staining in syncytiotrophoblast, and typically rare or negative expression in cytotrophoblast. MP demonstrates FOSB in the amnion, implantation site trophoblast, and cytotrophoblast in villi only near the fetal plate. All ISNs demonstrate strong FOSB staining in a subset of trophoblast which appears to correspond with intermediate-type trophoblast (as determined by MelCam IHC). FOSB was variable in CH-CA, with $9 / 10$ showing moderate staining in syncitiotrophoblast and cytotrophoblast; 4 diffusely and 5 focally. All PSTTs demonstrated strong diffuse FOSB staining, whereas all ETTs were only focally positive with weak to moderate staining patterns. All endometrial adenocarcinomas were negative for FOSB.

Conclusions: FOSB is strongly expressed in extravillous and implantation site trophoblast in early gestational tissues. Although not entirely specific, strong and diffuse expression of FOSB is sensitive for intermediate type trophoblast (in contrast to chorionic-type trophoblast) and PSTTs. In addition FOSB can help distinguish trophoblasts (neoplastic or non-neoplastic) from endometrial carcinomas, and this could be especially useful in biopsy specimens. Lastly, FOSB may be helpful in subtyping metastatic tumors of unknown origin when a trophoblastic neoplasm is in the differential diagnosis; however, further studies are needed to evaluate the specificity of FOSB in non-trophoblastic and non-endometrial tumors.

1212 Targeted Genomic Profiling and PD-L1 Expression in Epithelioid Trophoblastic Tumors and Placental Site Trophoblastic Tumors

Jelena Mirkovic, Elizabeth Garcia, Lynette M Sholl, Neal I Lindeman, Laura E MacConaill, Fei Dong, Bradley J Quade, Neil S Horowitz, Christopher P Crum, Brooke E Howitt. Brigham and Women's Hospital, Boston, MA.

Background: The pathogenesis of epithelioid trophoblastic tumors (ETT) and placental site trophoblastic tumors (PSTT) is not well understood. The aim of this study was to 1) examine the molecular alterations in ETTs and PSTTs to identify driver mutations and potential therapeutically targetable mutations, and 2) to evaluate the immunohistochemical expression of PD-L1 and characterize intratumoral lymphocytes in these tumors.
Design: 7 ETT, 7 PSTT, and one hybrid ETT/PSTT with available formalin-fixed paraffin embedded tumor were included. Genomic DNA was isolated and targeted next generation sequencing was performed to detect mutations, copy number variations and structural variants in exonic regions of 300 cancer genes and 113 selected intronic regions across 35 genes. PD-L1 immunohistochemistry and intratumoral CD3 and CD20+ lymphocytes were quantified in 5 ETT and 6 PSTT. Both intensity (0-negative, 1 -mild, 2-moderate, and 3-strong) and distribution ( $\%$ membranous staining) for PD$\mathrm{L} 1$ and highest number of $\mathrm{CD} 3+$ and $\mathrm{CD} 20+$ lymphocytes per high power field were recorded.

Results: No recurrent molecular aberrations were identified in ETTs and PSTTs. All PSTTs exhibited membranous expression of PD-L1 (average 63\%), the majority of which ( 4 of 6 ) were $2+$. All but one ETT exhibited $2+$ focal membranous expression of PD-L1, (average 16\%). ETTs had an average of 122 (range 0-406) CD3+ lymphocytes and 32 (range 0-85) CD20+ lymphocytes. PSTT had an average of 187.8 (range 73-365) CD3 + cells and 85.7 (range 0-144) CD20+ lymphocytes.

Conclusions: No significant recurrent molecular aberrations were identified in ETTs and PSTTs. Both ETT and PSTT are characterized by conspicuous membranous expression of PD-L1 and may benefit from immune checkpoint inhibitor therapy.

1213 The Utility of Molecular Characterization of Gynecologic Small Blue Round Cell Tumors: Report of Two EWSR1-ERG-Positive Ewing Sarcoma Cases

Nissreen Mohammad, Angela Goytain, Martin Jones, Shangguo Tang, Michael Brennan, Blake Gilks, Janessa Laskin, Tony Ng. University of British Columbia, Vancouver, BC, Canada; Jack Bell Research Centre, Vancouver, BC, Canada; BC Genome Sciences Centre, Vancouver, BC, Canada; McMaster University Medical Centre, Hamilton, ON, Canada; Niagara Health System, St. Catherines General site, ON, Canada; Vancouver General Hospital, Vancouver, BC, Canada; BC Cancer Agency, Vancouver, BC, Canada. Background: Ewing sarcoma (ES) is molecularly characterized by translocations most commonly resulting in fusion of the EWSR1 gene with FLI1 or ERG. Distinguishing ES from other sarcoma types is critical because it portends a specific course of aggressive systemic therapy. ES of the gynecologic tract is rare, with overlapping morphologic, immunohistochemical and molecular features with other pelvic small round blue cell tumors (SBRCTs). Here, we discuss two cases of gynecologic SBRCTs with EWSR1ERG fusion.

Design: Two cases of pelvic small round cell tumors were subjected to molecular characterization by a Nanostring-based sarcoma fusion gene assay developed in house. One case was also subjected to whole genome/transcriptome analysis through the local Personalized Oncogenomics project. Results were validated using FISH and RTPCR. Results: Case 1 was a 21-year-old female with a large ovarian tumor, and Case 2 a 27-year-old female with a large pelvic mass and extensive intra-abdominal and lung metastases. Both tumors were positive for CD99 and CAM5.2. Nanostring assay performed on Case 1 showed expression of the EWSR1-ERG fusion. The result was validated by FISH and RTPCR. Case 2 underwent whole genome/transcriptome analysis; the EWSR1-ERG fusion was identified along with various other molecular alterations, including a pathogenic STAG2 mutation commonly seen in aggressive cases of ES. EWSR1-ERG expression was validated using the Nanostring assay. Case 2 initially received radiation and gynecologic chemotherapy protocol with poor response. Once the final diagnosis was reached, she was switched to ES protocol and showed near complete response.

Conclusions: We report two cases of keratin-positive, EWSR1-ERG-positive Ewing sarcoma of the gynecologic tract; characterization of the fusion oncogene lead to correct diagnosis, which was not suspected prior to molecular analysis. Pelvic ES is rare, and identification of both fusion gene partners was needed to rule out other differential diagnoses, particularly desmoplastic small round cell tumor, as well as other gynecologic SRBCTs.

1214 Primary Angioleiomyoma of the Female Genital Tract: A Clinicopathologic Analysis of 20 Cases

Kristen E Muller, Amir Lagstein. University of Michigan, Ann Arbor, MI.

Background: Primary angioleiomyoma (ALM) of the female genital tract, a unique variant of leiomyoma, is a rare entity with less than 20 cases reported in the English literature. With the exception of hormone receptor positivity, they are morphologically and immunophenotypically similar to their soft tissue counterparts. Due to their rarity, clinical and pathological features of ALM in the female genital tract are not wellestablished. We aimed to characterize the clinical and morphologic spectrum of ALM in the gynecologic tract.

Design: Primary gynecologic ALM were identified through retrospective review of our pathology database and consult files over a 16-year period. Clinical-pathologic findings were obtained through electronic medical records and pathology reports.

Results: Twenty ALM of the female genital tract were identified (age $47.7 \pm 8.8$ ). The most common presentation included pelvic mass and abnormal uterine bleeding $(75 \%$ and $55 \%$, respectively). Anemia due to excessive bleeding was present in four patients. Malignancy was a pre-operative clinical consideration in six cases, mainly due to large size or unusual anatomic location. Specimens included hysterectomy alone $(\mathrm{n}=7)$, total abdominal hysterectomy and bilateral salpingo-oophorectomy $(n=8)$, endometrial curettings $(\mathrm{n}=2)$, cervical polypectomy $(\mathrm{n}=2)$, and myomectomy $(\mathrm{n}=1)$. Histopathologic examination revealed pure angioleiomyoma (spindle cell smooth-muscle tumors with prominent angiomatous proliferation of small and large, thick-walled vessels) in 18 cases, while two contained a prominent myxoid component. ALM were primarily unifocal $(\mathrm{n}=17,85 \%)$ and myometrial $(\mathrm{n}=12,60 \%)$, with a minority of cases located in the endometrial submucosa $(n=3)$, cervix $(n=2)$, ovary $(n=1)$, pelvic side wall $(n=1)$, and uterosacral ligament $(\mathrm{n}=1)$. Three cases contained multifocal disease, while five exhibited an extra-uterine component. Many ALM were exceptionally large, including 
three cases over $30 \mathrm{~cm}(13.6 \pm 12.9 \mathrm{~cm}$, range $1.1-45 \mathrm{~cm})$. When performed $(\mathrm{n}=14)$, immunohistochemistry revealed diffuse smooth muscle actin and desmin expression, CD34 expression in the vascular endothelium, focal CD10 expression, and low ki67. Conclusions: Pathologists should be aware of ALM of the female genital tract as this is an uncommon benign neoplasm which may be clinically mistaken for malignant gynecologic entities in nearly a third of cases due to its large size and propensity for extra-uterine involvement. Careful histologic examination to recognize the characteristic morphology of these tumors is essential in order to avoid misdiagnosis or overdiagnosis of malignancy.

\section{Central Nervous System-Type Neuroepithelial Tumors in} Ovarian Teratomas

Tricia Murdock, Fausto Rodriguez, Brigitte M Ronnett. The Johns Hopkins Institutions, Baltimore, MD

Background: Central nervous system (CNS)-type neuroepithelial tumors arising in ovarian teratomas are extremely rare. Recognition of these as somatic tumors distinct from immature elements of immature teratoma is important for tumor classification which can have therapeutic implications.

Design: All cases with a diagnosis of a CNS-type neuroepithelial tumor associated with ovarian teratoma were retrieved from our surgical pathology files and reviewed. Classification of the neuroepithelial component was performed by a neuropathologist based on criteria used for primary CNS tumors. Clinicopathologic features and any available immunohistochemical and molecular testing results were tabulated.

Results: Five cases were identified in patients with an age range of 14-31 years. These included 2 CNS-type embryonal tumors, 1 arising in immature teratoma and 1 arising in mature cystic teratoma, 1 medulloblastoma arising in mature cystic teratoma, 1 malignant glioneuronal neoplasm arising in immature teratoma, and 1 neurocytoma arising in a mature cystic teratoma. The CNS-type embryonal tumors expressed synaptophysin, were negative for GFAP, and had high Ki-67 proliferation indices. The malignant glioneuronal neoplasm expressed NeuN, GFAP, and synaptophysin, and SNP microarray analysis of chromosome $19 \mathrm{q}$ demonstrated two regions of deletion: (19) (q11q13.12) and (19)(q13.4qter). The neurocytoma expressed synaptophysin and ATX6, and per SNP microarray analysis there was no whole arm co-deletion of $1 \mathrm{p}$ and $19 \mathrm{q}$. Conclusions: CNS-type neuroepithelial tumors analogous to those primary in the CNS can arise in ovarian teratomas, both immature and mature cystic types. Recognition of the spectrum of tumors that can be encountered is important for distinction from immature teratoma elements for tumor classification and grading. Immunohistochemical and molecular analysis can assist in subclassification.

1216 Therapeutic Potential of Anti-CD137 in Ovarian Tumors

Alexander Nobori, Itsushi P Shintaku, Neda A Moatamed. David Geffen School of Medicine at UCLA, Los Angeles, CA.

Background: CD137 is a tumor necrosis factor receptor, which regulates the activation of many immune cells, including CD4, CD8 T cells, dendritic cells, and natural killer cells. It is a promising target for enhancing antitumor immune responses. It has been shown that CD137 ligand inhibits proliferation and induces apoptosis in some tumors like lung cancer. In this study, we investigated the expression of CD137 in human ovarian neoplasms

Design: We assessed CD137 expression on a tissue microarray of 223 normal and neoplastic ovarian tissues. Immunohistochemical staining of the tissues was carried out using anti-CD137 (rabbit polyclonal) antibody. Absent cytoplasmic staining of CD137 was scored as negative and present staining was scored as positive.

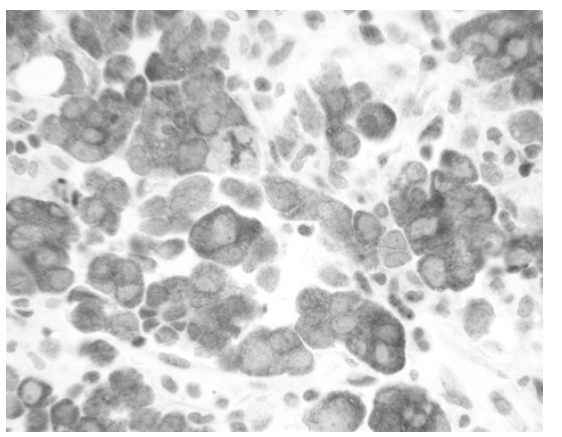

Results: Table 1 summarizes the frequency of CD137 positivity in ovarian tissues. Overall, CD137 was positive in $24 \%(42 / 173)$ of all ovarian malignancies. Subcategorically, $22 \%(10 / 46)$ of serous, $22 \%(2 / 9)$ of clear cell, $27 \%(12 / 44)$ of endometrioid, $25 \%$ (4/16) of mucinous, and $21 \%$ (3/14) of metastatic carcinomas, and $9 \%(1 / 11)$ of borderline tumors were positive. CD137 was also positive in $59 \%$ of germ cell tumors (10/17). All benign tumors as well as the normal ovary were negative for PD-L1 expression.

\begin{tabular}{|r|c|c|c|}
\hline Table 1 & \multicolumn{3}{|c|}{ CD137 Expression in Ovarian Tissue and Its Neoplasms } \\
\hline $\mathrm{n}=223$ & $\mathrm{n}$ & Expressed (n) & Expression (\%) \\
\hline Malignant Tumors & 173 & 42 & $24 \%$ \\
\hline Serous CA & 46 & 10 & $22 \%$ \\
\hline Clear Cell CA & 9 & 2 & $22 \%$ \\
\hline Endometrioid CA & 44 & 12 & $27 \%$ \\
\hline Undifferentiated CA & 3 & 0 & $0 \%$ \\
\hline Mucinous CA & 16 & 4 & $25 \%$ \\
\hline Metastatic CA & 14 & 3 & $21 \%$ \\
\hline Squamous Cell CA & 1 & 0 & $0 \%$ \\
\hline Germ Cell & 17 & 10 & $0 \%$ \\
\hline MMMT & 3 & 0 & $0 \%$ \\
\hline Sarcoma & 3 & 0 & $0 \%$ \\
\hline Sex-Cord & 15 & 0 & $0 \%$ \\
\hline Small Cell CA & 1 & 0 & $0 \%$ \\
\hline Lymphoma & 1 & 0 & $9 \%$ \\
\hline Borderline Tumors & 11 & 1 & $0 \%$ \\
\hline Benign Tumors & 29 & 0 & \\
\hline Normal Ovary & 10 & 0 & \\
\hline CA, carcinoma; MMMT, mixed müllerian malignant tumor & \\
\hline
\end{tabular}

Conclusions: This study shows significant expression of CD137 in 24\% of ovarian malignancies. Positive tumors may serve as potential candidates for the anti-CD137 immunotherapies.

1217 Biopsies of Papillary Squamous Cell Carcinoma of the Uterine Cervix Frequently Underrepresent the Stromal Invasion Seen in the Excisional Specimens

Atousa Ordobazari, Mehrdad Nadji, Marilyn Huang, Andre Pinto. University of Miami, Miami, FL.

Background: Papillary squamous cell carcinoma (PSCC) of the uterine cervix is defined by the World and Health Organization as "a tumor in which thin or broad papillae of connective tissue stroma are covered by epithelium showing the features of high grade squamous intraepithelial lesions (HSIL)". Due to the exophytic nature of these neoplasms, the assessment and quantification of invasion may be challenging in small biopsies, as these samples often do not contain sufficient amount of stroma for evaluation. The goal of this study was to determine the presence and dimensions of stromal invasion, if any, in the original biopsies compared to an excisional specimen. Design: All cases with the diagnosis of papillary and/or exophytic squamous cell carcinomas of the cervix between 2003 and 2016 were retrieved from the files of the department of Pathology at the University of Miami, Jackson Memorial Hospital. Data from the original biopsies and corresponding resection specimens, including the presence, depth, and horizontal extent of stromal invasion were recorded.

Results: A total of 48 cases of diagnostic biopsies and 26 matching resections were identified (excisional data was unavailable for 22 patients). Patient age ranged from 21 to 79 years (mean: 48.9; median: 50). Among all pre-surgical biopsies, the presence and assessment of depth of invasion (and/or horizontal extent) was diagnosable in only $4 / 48(8.3 \%)$. As expected, most original biopsy reports contained a disclaimer stating the limitations of the sample for such evaluation. In the surgical excision specimens ( 22 hysterectomies and 4 cone biopsies), on which 23 of the 26 cases were invasive carcinomas, the ability to accurately provide at least one of the two parameters (depth and/or linear extent) increased to $23 / 23(100 \%)$. Tumor sizes ranged from 0.5 to $6.6 \mathrm{~cm}$; stromal invasion ranged in depth from $0.1 \mathrm{~cm}$ to $2.2 \mathrm{~cm}$ (median: 1), and in horizontal length from 2 to $4.5 \mathrm{~cm}$ (median: 2 ).

Conclusions: The diagnosis and assessment of invasion in superficial biopsies of PSCC is extremely challenging. However, resection specimens following small, superficial samples (and are often the ones that will guide the surgical approach) most often contain established stromal invasion. Communication with the clinical team is fundamental to guide proper management, and avoid under-treatment in patients with such lesions undergoing limited biopsies.

1218 Canadian Consensus Guidelines for Benign Endometrial Biopsy Reporting: Development and Internal Validation

Carlos Parra-Herran, Matthew Cesari, Bojana Djordjevic, Katherine Grondin, Mary Kinloch, Martin Koebel, Amrah Pirzada, Anna Plotkin, C Blake Gilks. Sunnybrook Health Sciences Centre, Toronto, ON, Canada; Centre Hospitalier Universitaire de Quebec, Quebec, QC, Canada; Saskatoon City Hospital, Saskatoon, SK, Canada; University of Calgary, Calgary, AB, Canada; Memorial University, St John's, NL, Canada; Trillium Health Partners, Toronto, ON, Canada; Vancouver General Hospital, Vancouver, BC, Canada.

Background: Standardized pathologic reporting of endometrial cancer allows for consistent patient diagnosis and management, and facilitates quality assurance. Reporting of benign diagnoses in endometrial biopsy currently lacks such standardization, and updated consensus terminology is needed. The Special Interest Group in Gynecologic Pathology of the Canadian Association of Pathologists has embarked in the elaboration of national guidelines for benign endometrial biopsy reporting. Our aim was to validate the guideline through its utilization in routine biopsy evaluation.

Design: A group of 9 pathologists with expertise in gynecologic pathology and nationwide representation developed a list of the most frequent and relevant benign diagnoses encountered in endometrial biopsy. Explanatory notes and relevant references 
were also included. Five pathologists applied the guideline to all benign endometrial biopsies in their practices over a one-week period; biopsies with malignant or pre-malignant lesions were excluded. Number of cases in which the guideline was successfully used was recorded, as well as instances in which the guideline could not be applied satisfactorily and the diagnosis had to be recorded as free-text.

Results: The one-page consensus document included 20 possible diagnostic terms; 5 of these including optional additional information lines. A total of 212 benign endometrial biopsies were evaluated and reported following the guideline. The recommended terminology adequately incorporated the diagnosis in 203 cases $(95.8 \%)$. In the remaining nine, the guideline could not be applied because the final diagnosis was not fully incorporated into the consensus list (5 cases, $2.3 \%$ ) or due to the presence of relevant non-endometrial tissue diagnoses such as leiomyoma, myometrium and cervical tissue (4 cases, $1.9 \%)$.

Conclusions: Based on our validation results, a list of unified terminology for benign endometrial biopsy reporting can successfully cover the vast majority of diagnostic scenarios. This initial validation step demonstrates that standardized reporting can be applied to benign cases and potentially improve patient care, quality assurance and research initiatives. It also provides further information to incorporate uncommon, yet important diagnoses into the guideline.

\section{BMI and Differential Epithelial and Stromal ER/PR Expression} within Type I and Type II Endometrial Carcinoma

Joseph Peevey, Kruti P Maniar, Ji-Yong Julie Kim. Northwestern Memorial Hospital, Chicago, IL.

Background: Expression of estrogen (ER) and progesterone (PR) receptors on endometrial stromal cells is critical in modulating the differentiation, function, and proliferation of endometrial epithelial cells via reciprocal paracrine signaling. Stromal $\mathrm{ER} / \mathrm{PR}$ expression status has been shown to influence endometrial cancer (ECa) tumor volume and response to hormone treatment in-vitro. Recent studies found no relationship of hormone treatment response with either BMI or ER/PR expression in tumor epithelium; however these studies did not evaluate stromal hormone expression. Design: The aim of this study was to further characterize the relationship between BMI and epithelial and stromal ER/PR expression in Type I vs Type II ECa. Sections from tissue microarrays (TMA) were used for ER/PR immunohistochemical evaluation and scored by the percent of positive cells $[(0)$ absent; (1) $<10 \%$; (2) $11-50 \%$; (3) $>50 \%]$. TMA was constructed using $0.2 \mathrm{~cm}$ cores with positive/negative control tissue $(90$ total patients). Relevant clinco-pathologic data was recorded (Age, BMI, Tumor Type/Grade/ Stage, etc.). Patient populations were separated based upon tumor type (Type I vs Type II) and BMI $\geq$ or $<40$. $p$ values $\leq 0.05$ were considered significant.

Results: As expected, independent of BMI, significantly greater tumor epithelial expression of ER and PR is seen in Type I as compared to Type II. Also significant and independent of BMI, Type I stroma more frequently expressed ER and PR than Type II stroma. Interestingly, epithelial PR expression is significantly increased in Type I $\mathrm{ECa}$ with $\mathrm{BMI} \geq 40$ as compared to $\mathrm{BMI}<40$.

Conclusions: Independent of BMI, significant differences in ER and PR expression are seen within both epithelium and stroma when comparing Type I vs Type II ECa. BMI $\geq 40$ is associated with significantly increased PR expression within Type I ECa epithelium, but not within stroma. These findings have possible clinical implications for treatment consideration based upon BMI as it is possible that patients with Type I ECa and low BMI will not respond as well to hormone treatment. Hormone therapy is commonly used in patients who desire to preserve future fertility or who are otherwise not operative candidates. Currently, ER/PR receptor expression is only reported in carcinoma cells. These results raise consideration of scoring background stroma as well and also taking patient BMI into consideration when counseling hormone treatment options.

1220 Acantholytic Squamous Cell Carcinoma of the Uterine Cervix Delia Perez Montiel, Hugo Dominguez, Guadalupe Moncada, Lourdes Pena, David Cantu de Leon. Instituto Nacional de Cancerologia, Mexico City, Mexico.

Background: Acantholytic variant of squamous cell carcinoma (ASCC) is characterized by discohesive cells that can give a pseudoglandular and/or angiomatoid pattern to the tumor. It has been considered of poor prognosis in certain anatomic sites such as head and neck and vulva. In the uterine cervix, the importance of this variant has been barely described.

Design: A ten year retrospective review of squamous cell carcinoma of the uterine cervix was performed to identify cases of ASCC and was correlated with clinical characteristics and prognosis. Cases were matched with controls ( non- acantholytic, squamous cell cervical cancer). To confirm squamous differentiation immunostains for keratin 5/6, keratin 14 and P63 were performed when indicated and had to be positive at least for two stains. All patients were treated according to the clinical stage with radical surgery or chemo radiation.

Results: During the study period 20 cases with areas of ASCC were identified from a total of 2438 cases (less than 1\%). ASCC areas accounted for 10 to $80 \%$ of the tumor. No cases of pure ASCC were founded. In $6(30 \%)$ cases the pattern was pseudoglandular, angiomatoid in $4(20 \%)$, acantholytic cell nest $4(20 \%)$, combined pattern $6(30 \%)$. Mean age at diagnosis was 51 years (range 36-81). Clinical stages were $1 \mathrm{~A} 2$ (1 case), Ib1 (14), IIB (2), IIIB (3), IVB (1). In those patients initially treated with radical hysterectomy 12 had notable lymphovascular space permeation irrespective of depth of invasion and 2 patients had lymph node metastasis ( 9 and 13 positive lymph nodes). Median follow-up was 64 months (range $12-120$ ). When comparing with controls no statistical differences were identified in rate of local recurrence or metastatic disease,only one patient with ASCC and lymph node metastasis in radical hysterectomy developed lung metastasis after 10 years, the rest of the patients are alive, without local or distance recurrence. Conclusions: Acantholytic areas in squamous cell cervical cancer are infrequent and because pseudoglandular and angiomatoid pattern the differential diagnosis must include endocervical and non endocervical adenocarcinomas. In contrast to other sites ASCC, it seems not to have implication in the prognostic of patients with this specific histologic variant.

\section{Uterine Rhabdomyosarcoma in Adults: A Clinicopathologic} Study of Five Cases

Andre Pinto, Ryan Kahn, Darcy A Kerr, Andrew E Rosenberg, Brian Slomovitz, Breelyn Wilky, Marilyn Huang. University of Miami, Miami, FL.

Background: Rhabdomyosarcoma (RMS) accounts for only 5-10\% of all sarcomas in adults. Primary uterine RMS comprises an even more restricted subset, with very little known when compared to most other gynecologic malignancies. These tumors are usually found as a component of uterine carcinosarcomas, whereas "pure" primary RMS are exceedingly rare.

Design: We retrospectively searched our pathology database for all cases of RMS involving the uterus. Cases without a definitive diagnosis, cases where RMS comprised a component of a carcinosarcoma and/or cases occurring in children and adolescents were not included. Clinical history (including follow-up) was obtained, and slides were reviewed by two pathologists. Immunohistochemical profile and molecular studies, when available, were included.

Results: A total of 5 cases fulfilled inclusion criteria. They corresponded to tumors occurring in patients from 22 to 68 years of age (median: 62). The most common presentation among all patients was heavy vaginal bleeding. Tumor size ranged from $8.1-15 \mathrm{~cm}$ (median: 13.3). Four occurred in the corpus while one arose in the cervix. The most common subtype was pleomorphic RMS (3/5), one alveolar RMS (1/5) and one was embryonal RMS. Review of referring diagnoses showed that most cases were initially either misdiagnosed or included in the differential diagnosis among other malignant tumors such as high-grade endometrial stromal sarcoma (HG-ESS), undifferentiated uterine sarcoma (UUS) or carcinosarcoma. All cases demonstrated positive immunohistochemical staining for Desmin (5/5) and Myogenin (5/5), and all three cases that were tested for Myo-D1 were also positive. The alveolar RMS case demonstrated the characteristic $\mathrm{t}(2 ; 13)$ by molecular studies. All patients presented with advanced stage disease (IVB), except for one (inoperable; therefore, surgical staging not available). Despite short follow up (all diagnosed within last 18 months), 3 patients are alive with disease (AWD) and receiving chemotherapy (either active or for maintenance), one patient is undergoing workup and one patient died of disease.

Conclusions: Uterine RMS is an exceedingly rare malignant neoplasm; patients present with very advanced and often widespread disease at time of diagnosis. Given its rarity, these tumors are often misdiagnosed, so awareness of this entity in the female genital tract is important in order to establish a correct diagnosis. The diagnosis can be facilitated with a relatively limited panel of immunohistochemical studies.

1222 Frequent Non-Syndromic Deletion and Transcript Reduction of COL4A5 and COL4A6 in Uterine Leiomyosarcoma

Bradley J Quade, Liping Yuan, Marisa R Nucci, Brooke E Howitt. Brigham and Women's Hospital and Harvard Medical School, Boston, MA.

Background: Deletion of fumarate hydratase in hereditary leiomyomatosis-renal cell carcinoma syndrome provides an established example of smooth muscle neoplasia driven by heritable factors. A less well understood heritable syndrome predisposing to smooth muscle neoplasia is Diffuse Leiomyomatosis with Alport syndrome (DL-ATS). DL-ATS is a contiguous gene deletion syndrome that occurs when the shared promoter regions of COL4A5 and COL4A6, arranged head-to-head on chromosome X (chr X), are deleted. DL-ATS results in leiomyomatosis of the vulva, esophagus and tracheobronchial tree. These non-fibrillar type IV alpha collagen chains are highly expressed by myometrial cells. This study investigates non-syndromic deletion of COL $4 A 5$ and COL4A6 in uterine smooth muscle tumors, focusing first on leiomyosarcomas and atypical leiomyomas.

Design: DNA and RNA was isolated from FFPE tissue blocks of 68 leiomyosarcoma (LMS) samples from 46 subjects, and 60 atypical leiomyomas (AL) or smooth muscle tumors of uncertain malignant potential (STUMP). COL $4 A 5$ and COL4A6 copy number abnormalities were evaluated by array Comparative Genomic Hybridization (aCGH, index case) and Multiple Ligation-dependent Probe Amplification (MLPA, all cases). RNA expression was performed previously by microarray profiling (Affymetrix) and currently by hybridization and bar-coded fluorescent tagging (Nanostring).

Results: An index case in which an AL preceded LMS by 4 yr was analyzed by aCGH. Both tumor samples shared deletions of $p 53, R B 1$, and a small chr X segment involving the promoters and 5' regions of COLAA5 and COL4A6, but the LMS had a unique deletion of chromosome 10. MLPA analysis of the entire cohort showed COL4A5 and COL4A6 in $19.6 \%$ of LMS, but in no AL studied to date. The deletions were limited to the collagen or nearby genes on chrX in 4 LMS tumors, but encompassed a large portion of chr $\mathrm{X}$ in $5 \mathrm{LMS}$ tumors. Expression profiling showed that mRNA levels for COL4A5 and COL4A6 were reduced by 2.7 and 14.3 -fold, respectively. Transcript reduction was much more pronounced in LMS tumors with gene deletion.

Conclusions: Deletion of COL $4 A 5$ and COL4A6 occurs in $~ 20 \%$ of LMS, and results in loss of expression of these genes. The minimal deletion region noted in a subset of LMS tumors was similar to the region lost in DL-ATS. Interestingly, the non-collagenous domains of the non-fibrillar type IV collagen family members have anti-tumor properties, raising the possibility that their loss in uterine LMS promotes their tumor growth. 
1223 Frequency of Rare and Multi Viral High-Risk HPV Subtype Infection in Cervical Squamous and Glandular Lesions in an Immigrant Dominant Middle Eastern Country: A PCR-Based Study

M Ruhul Quddus, Alia Albawardi, Shamsa Al-Awar, Saeeda SH Almarzooqi. Women \& Infants Hospital/Alpert Medical School of Brown University, Providence, RI; College of Medicine and Health Sciences, UAE University /Tawam Hospital, Al Ain, Abu Dhabi, United Arab Emirates; College of Medicine and Health Sciences, UAE University, Al Ain, Abu Dhabi, United Arab Emirates.

Background: Cervical cancer is among the top 3 cancers in the UAE.The reported incidence of abnormalities in cervical screening in the UAE is 3.6\%. Data regarding specific HPV genotypes are lacking in this population.Currently women receive the quadravalent vaccine. Identification of specific high risk HPV (hrHPV) types will direct local screening \& vaccination guidelines.

Design: A total of 75 archival cervical cone biopsies with HSIL or higher lesions(20122016), were retrieved from a tertiary hospital. Diagnoses were HSIL $(n=70)$, adenocarcinoma in-situ( $(\mathrm{n}=1)$ and invasive carcinoma(n=3). Five tissue sections $(10-$ $\mu$-thick) were cut. DNA extraction was done using the QIAamp DNA FFPE Tissue Kit (QIAGEN Inc,Valencia, CA 91355, Cat No. 56404). GenomeMe ${ }^{\mathrm{TM}}$ 's GeneNav ${ }^{\mathrm{TM}}$ HPV One qPCR Kit (GenomeMe, Richmond, BC, Canada) for specific detection of HPV 16\&18; non-16/18 samples were typed by GenomeMe ${ }^{\mathrm{TM}}$ 's GeneNav ${ }^{\mathrm{TM}} \mathrm{HPV}$ Genotyping qPCR Kit.

Results: Median age for HSIL \& higher lesions was 34 yrs (19-58 years). UAE nationals were $70 \%$ of cases. Overall, HPV types were $16,18,31,33,35,39,45,51,52,58,59$, $66 \& 68$. Nine of $70(13 \%)$ HSIL cases were negative for hrHPV. Most common types were HPV 16 (49\%), HPV 31 (20\%) \& HPV $18(7 \%)$.

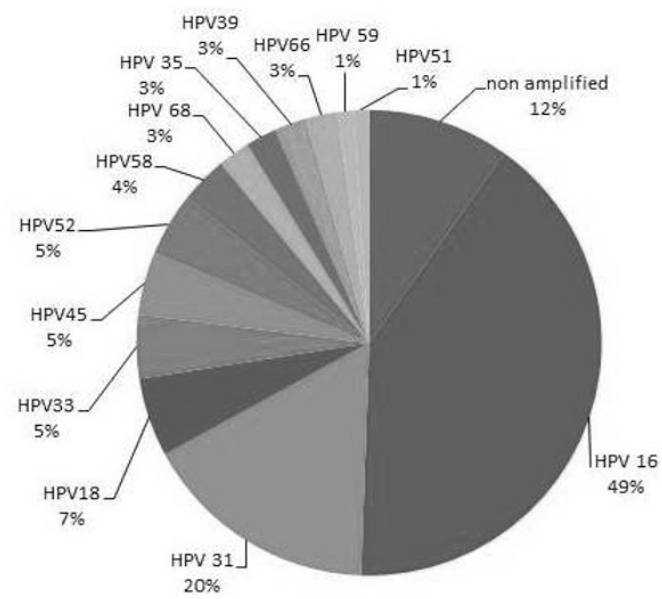

HPV $16 / 18$ identified in $56 \%$ and other types in $32 \%$. Co-infection was seen in 12/75(16\%).Eight cases had 2 multi-viral types \& 4 had 3 infections. Multi-viral HVP infection was limited to HPV 16,18 \& 31 cases.

Conclusions: Infection by non HPV 16/18 is fairly common. hrHPV31 was detected in $20 \%$. Multi-viral hrHPV was encounter in 16\%. The current findings are significant for this region where the quadravalent vaccine is used. Results will impact future screening and prevention strategies. A larger study appears prudent to ratify our findings.

\section{Endometrial Carcinoma with Trophoblastic Differentiation: A} Clinicopathologic Analysis of $\mathbf{2 4}$ New and Previously Reported Cases

Kojo Rawish, Natalia Buza, Wenxin Zheng, Oluwole Fadare. Vanguard Pathology Associates, Austin, TX; Yale University, New Haven, CT; UTSW, Dallas, TX; UCSD, San Diego, CA.

Background: Trophoblastic differentiation is rare in endometrial carcinoma. To better characterize this enigmatic phenomenon, we report herein 4 new cases, representing the largest series reported thus far, and analyze previously reported cases

Design: The 4 new cases were assembled from multiple institutions. A literature search yielded 20 additional cases, reported between 1972 and 2016

Results: Our patients ranged in age from 61 to 77 years (mean 68). Patient \#1 had a FIGO grade 1 endometrioid carcinoma (EEC), surgically FIGO staged IA, that recurred 5 months later at the vaginal apex with purely trophoblastic elements, suggestive of unsampled trophoblastic areas in the original evaluation. Other metastases ensued, and she was dead of disease (DOD) 13 months after primary staging. Patients 2 and 3 had dedifferentiated carcinomas (DDC) with large $(40 \%, 20 \%)$ trophoblastic areas; both were initially FIGO staged III by virtue of adnexal involvement in patient 2 and pelvic lymph node involvement in patient 3 , and both patients were DOD at 7 and 16 months respectively. The fourth patient had a FIGO grade 1 EEC, stage III, with a $60 \%$ trophoblastic component. She was DOD at 11 months. The trophoblastic areas in all cases, if viewed in isolation, were diagnostic of choriocarcinoma. The extrauterine foci were either the trophoblastic elements or the DDC component in all cases with evaluable material. Combining our data with reported patients in the literature, the total of 24 patients ranged in age from 42 to 85 years (mean 65). Associated histotypes included EEC ( $42 \%$ ), serous carcinoma (13\%), carcinosarcoma ( $8 \%$ ), DDC (8\%), EEC mixed with serous and/or clear cell carcinoma ( $8 \%$ ), and adenocarcinoma NOS (21\%). $78 \%$ of the patients had extrauterine disease at presentation, and the lungs and regional lymph nodes were the most common metastatic sites. Pre- and/or postoperative serum/ urine $\beta$-hcG were elevated in $94 \%$ of cases where it was measured. On follow-up (F/U), $54 \%$ were DOD, $17 \%$ were alive with disease, and $21 \%$ showed no evidence of disease ( $\mathrm{F} / \mathrm{U}$ absent in $8 \%)$.
Conclusions: Endometrial carcinoma with trophoblastic elements are rare neoplasms that occur primarily in postmenopausal patients, are usually, but not invariably advanced stage at presentation, and are associated with poor prognosis. The diversity of histotypes with which they are associated suggests that trophoblastic differentiation may represent a form of dedifferentiation in endometrial carcinomas

\section{5}

\section{Carcinomas}

Opal L Reddy, Josephine S Aguilar-Jakthong, Itsushi P Shintaku, Neda A Moatamed. David Geffen School of Medicine at UCLA, Los Angeles, CA.

Background: Programmed death ligand 1 (PD-L1) is a transmembrane protein involved in immune responses. PD-L1 binds its receptor PD-1 on T cells to regulate T-cell activation and block anti-tumor immunity. The PD-1/PD-L1 axis has emerged as a promising new target for cancer therapeutics, particularly in the treatment of lung carcinoma and melanoma. In this study, we investigated the expression of PD-L1 in human endometrial tissue and endometrial tumors.

Design: PD-L1 expression was evaluated by immunohistochemistry performed on a tissue microarray of 98 normal, hyperplastic, and neoplastic endometrial tissues. Hematoxylin \& eosin and PD-L1 immunohistochemical staining (clone SP142, rabbit monoclonal antibody) were performed. PD-L1 expression was scored as positive when present on cell membranes (Figure 1), regardless of percent positivity, and negative when absent.

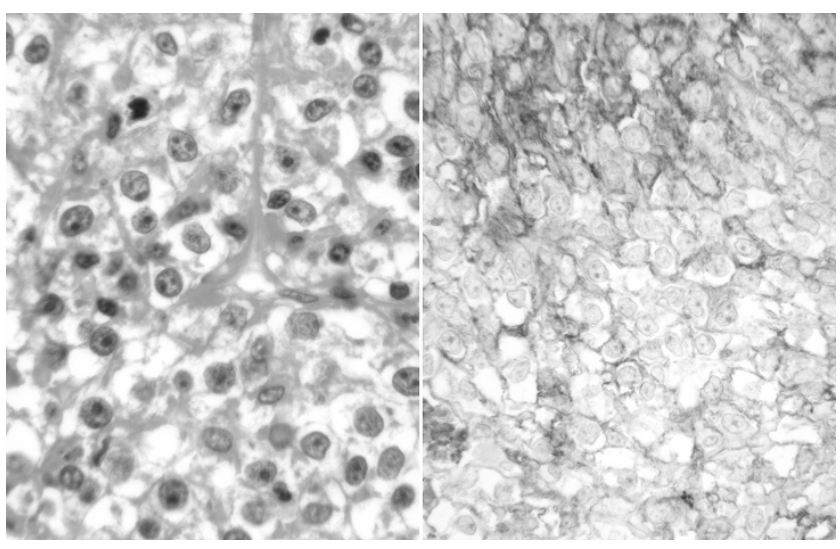

Results: Table 1 summarizes the frequency of PD-L1 positivity in 98 endometrial tissues. Overall, PD-L1 was positive in $27 \%$ (25/91) of endometrial malignancies. Sub-categorically, 26\% (21/82) of endometrioid adenocarcomas, $100 \%(3 / 3)$ of clear cell carcinomas, and $17 \%(1 / 6)$ of serous carcinomas were positive for PD-L1. Of the endometrioid adenocarcinomas, $86 \%(6 / 7)$ of tumors with squamous differentiation showed PD-L1 expression. PD-L1 was negative in five normal endometrial samples and two samples of endometrial hyperplasia.

\begin{tabular}{|l|c|c|c|}
\hline Table 1 & \multicolumn{3}{|c|}{ PD-L1 Expression in Endometrium and its Neoplasms } \\
\hline $\mathrm{n}=98$ & $\mathrm{n}$ & Expression (n) & Expression (\%) \\
\hline Malignant Tumors & 91 & 25 & $27 \%$ \\
\hline Endometrioid & 82 & 21 & $26 \%$ \\
\hline Endometrioid - Sq & 75 & 15 & $20 \%$ \\
\hline \multicolumn{1}{|c|}{ Endometrioid + Sq } & 7 & 6 & $86 \%$ \\
\hline Serous & 6 & 1 & $17 \%$ \\
\hline Clear Cell & 3 & 3 & $100 \%$ \\
\hline Hyperplasia & 2 & 0 & $0 \%$ \\
\hline Normal & 5 & 0 & $0 \%$ \\
\hline Sq, squamous differentiation; -, without; +, with \\
\hline
\end{tabular}

Conclusions: This study shows significant expression of PD-L1 in $27 \%$ of the endometrial malignancies, suggesting a role for future investigation of anti-PD-L1/PD-1 immunotherapies in the treatment of PD-L1-positive endometrial tumors.

1226 Poor Inter-Rater Agreement in the Diagnosis of Intra-Epithelial Fallopian Tube Nuclear Atypia

Nicholas P Reder, Nicholas Agoff, Rochelle Garcia, Elizabeth Swisher, Mark R Kilgore. University of Washington Medical Center, Seattle, WA; Virginia Mason Hospital and Medical Center, Seattle, WA.

Background: Intra-epithelial fallopian tube neoplasia is thought to be the precursor lesion to many cases of pelvic serous carcinoma, particularly in $B R C A 1$ and $B R C A 2$ mutation carriers. This association has led to recommendations to assess fallopian tubes for intra-epithelial atypia (PMID: 14668548). However, definitive criterion for fallopian tube epithelial atypia (referred to by some as Serous Tubal Intraepithelial Lesion or STIL) are not well-established and diagnostic reproducibility has not been rigorously studied. We hypothesized that mild and moderate atypia would have low inter-rater agreement, while severe atypia and invasive carcinoma would have good inter-rater agreement. Design: Two experienced gynecologic pathologists evaluated cross-sections of fallopian tubes from a cohort of women $(\mathrm{N}=220)$ undergoing salpingectomy. The cohort was enriched for women with BRCA mutations. In total, 619 slides were available for analysis, with multiple fallopian tube sections on each slide. Cohen's kappa was used to measure inter-rater agreement. All analyses were performed using R v3.3.1. 
Results: The presence of fallopian tube atypia was rare within this cohort (mild 6.6\%, moderate $1.3 \%$, severe $1.3 \%$ ) and carcinoma was exceedingly rare $(0.9 \%)$, see Table 1. Inter-rater agreement was no better than chance for mild (kappa $=0.07)$ and moderate $(\mathrm{kappa}=-0.01)$ atypia. Agreement for severe atypia was poor $(\mathrm{kappa}=0.25)$. Cases with carcinoma showed good inter-rater agreement (kappa $=0.83$ ).

\begin{tabular}{|l|c|c|c|}
\hline Variable & Pathologist 1 & Pathologist 2 & Overall \\
\hline Mild Nuclear Atypia & $9.0 \%$ & $4.2 \%$ & $6.6 \%$ \\
\hline Moderate Nuclear Atypia & $1.1 \%$ & $1.6 \%$ & $1.3 \%$ \\
\hline Severe Nuclear Atypia w/o Stratification & $0.3 \%$ & $2.2 \%$ & $1.3 \%$ \\
\hline Invasion & $1.1 \%$ & $0.8 \%$ & $0.9 \%$ \\
\hline
\end{tabular}

Conclusions: The diagnosis of mild, moderate, and severe atypia within the fallopian tube epithelium is hindered by low inter-rater agreement. Cases with invasion have good inter-rater agreement. These findings do not support the validity of a diagnosis of fallopian tube nuclear atypia without stratification, especially in cases with only mild to moderate atypia.

1227

Endometrial Serous Carcinogenesis in a Transgenic Mouse

Model

Glorimar Rivera-Colon, Li Li, Wenjing Zhang, Setsuko Chambers, Beihua Kong, Wenxin Zheng. University of Texas Southwestern Medical Center, Dallas, TX; Shandong University, School of Medicine, Jinan, Shandong, China; Qilu Hospital, Shandong University, Jinan, Shandong, China; University of Arizona, Tucson, AZ.

Background: The aim of this study is to examine if the ESC developmental process in the human can be reproduced in the mouse by selectively knockout TP53 in genitourinary system.

Design: This transgenic mouse model was established by using a combinational system of Cre-loxP and Ksp1.3 promoter to make sure TP53 was selectively knocked out in the target organs. In both target $\left(\operatorname{Trp} 53^{\Delta / \Delta}\right)$ and control $\left(\operatorname{Trp} 53^{\mathrm{f} / \mathrm{f}}\right)$ mice, organs from genitourinary system including uterus and fallopian tubes were analyzed every 2 weeks, starting from week 10 and ending at week 80 .

Results: ESC developed successfully in the uteri of the target mice and the sequence of ESC development in the human was completely reproduced in the mouse model. Serous endometrial precancer, endometrial glandular dysplasia (EmGD) was first seen at the age of 12 weeks, while the first serous endometrial intraepithelial carcinoma (SEIC) was found at 32 weeks and ESC at 66 weeks with the ESC penetrance rate of $100 \%$. All lesions in the process of ESC development were morphologically identifiable and immunohistochemically confirmed. Although Trp53 was knocked out in other genitourinary organs in addition to the endometrium, no gross and microscopic lesions were found in the fallopian tubes, ovaries, kidneys, and bladders.

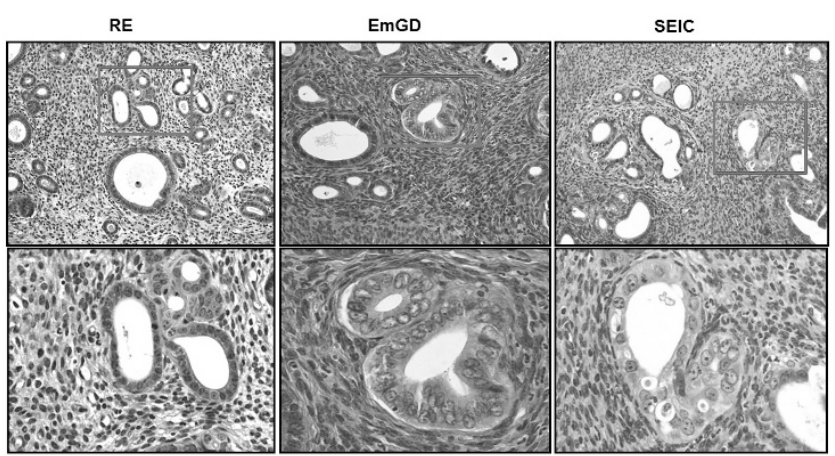

Conclusions: We conclude that the mouse model produces ESC reliably in mice. It simulates and confirms the entire process of what is known about endometrial serous carcinogenesis in the human. This model is useful for further studies of ESC pathogenesis at the molecular level, cancer early detection, prevention, and clinical intervention. It is also interesting to note that development of tubal high-grade serous carcinoma is apparently different from that of ESC.

\section{Yes-Associated Protein-1 (YAP) Is Preferentially Overexpressed} in High Grade Endometrial Carcinomas

Brian Robinson, Anthony Martinez, Anne Mills, Cynthia Cohen, Krisztina Z Hanley. Emory University, Atlanta, GA; University of Virginia, School of Medicine, Charlottesville, VA.

Background: The transcription factors YAP and Transcriptional Coactivator With PDZ-Binding Motif (Taz) are paralogs and transcriptional coactivators of the Hippo tumor suppressor pathway, a pathway implicated in numerous gynecologic malignancies. Recent studies have shown that increased nuclear expression of YAP and TAZ is associated with high-grade endometrial carcinomas (EC), adverse pathologic findings, and are independent prognostic factors for overall survival. YAP was found to be coactivator and modulator of transcriptional activity of ER and PR. While studies have examined TAZ expression by immunohistochemistry (IHC) in various subtypes of high-grade EC, characterization of YAP expression in different histologic variants of high-grade EC remains incomplete.

Design: Tissue microarray and whole tissue sections from 21 complex atypical hyperplasia $(\mathrm{CAH})$ and $95 \mathrm{EC}$ samples and were analyzed by IHC for YAP overexpression (Novus Biologicals, Littleton, CO). EC cases included 22 welldifferentiated endometrioid carcinomas, 25 FIGO grade 3 endometrioid carcinomas,
8 endometrial intraepithelial carcinomas (EIC), 13 serous carcinomas, 11 clear cell carcinomas and 16 carcinosarcomas. Results and staining intensity were evaluated in both nucleus and cytoplasm and scored on a 0-3 scale.

Results: Strong nuclear staining was observed in $7 / 22$ (32\%) of FIGO grade 1 endometrioid carcinomas, in 9/25 (36\%) of FIGO grade 3 endometrioid carcinomas, $6 / 8$ (75\%) of EIC, 10/13 (77\%) of serous carcinomas, 4/11 (36\%) of clear cell carcinomas and 11/16 (69\%) of carcinosarcomas. Overall 40/73 (54.8\%) of the high-grade EC shows strong nuclear YAP expression. CAH showed YAP expression in 7/21 (33\%) cases. Conclusions: High-grade EC, especially the more aggressive subtypes (serous, carcinosarcoma) express YAP more frequently than the endometrioid types confirming that inactivation of Hippo signaling is a frequent event in these neoplasms. Given the established role of YAP in modulating mutant-p53 activity in other tumors, it is likely that YAP expression has a functional role in the pathogenesis of these aggressive tumors. $\mathrm{CAH}$ and FIGO grade 1 endometrioid carcinomas showed similar, but lower rate of YAP expression, suggesting that YAP may play a role in estrogen driven preneoplastic and neoplastic lesions of the endometrium via a different pathway involving steroiddependent mechanisms.

\section{Patient-Derived Orthoxenografts (PDX) Reproduce Pathological} Features of Corresponding Endometrial Carcinomas (EC)

Nuria Ruiz, August Vidal, Jordi Ponce, Lola Marti, Jordi Rovira, Marc Barahona, Josep Maria Piulats, David Llobeta-Navas, Maria Santacana, Alberto Villanueva, Xavier Matias-Guiu. Hospital Universitari de Bellvitge-IDIBELL, L'Hospitalet de Llobregat, Barcelona, Spain; Hospital Universitari Arnau de Vilanova, Lleida, Spain; Institut Català d'Oncologia-IDIBELL, L'Hospitalet de Llobregat, Barcelona, Spain; Business Bioincubator, Bellvitge Health Science Campus, L'Hospitalet de Llobregat, Barcelona, Spain.

Background: PDX may be good tools for assessment of tumor progression and evaluation of adjuvant therapy.

Objectives: To establish PDX models using human EC of different types: Endometrioid (EEC), Serous (SC), Clear cell (CC), Mixed (MC), Undifferentiated/Dedifferentiated (UC) carcinomas and Carcinosarcomas (CS). To check the ability of PDX to reproduce the main pathologic and immunohistochemical (IHC) features of human tumors.

Design: 93 EC patients were included (55 EEC, 10 SC, 5 CC, 5 MC, 4 UC, 14 CS) Fresh tumor tissue was implanted in the uterus of immunocompromised mice. When tumor growth was obvious by palpation, mice were euthanized and tumor tissue was frozen and subjected to formalin-fixed, paraffin-embedding (FFPE). For IHC analysis, a Tissue Microarray was built with two cylinders from each FFPE human and matched xenograft tumor from the first 42 cases. IHC panel included: Estrogen Receptor (ER), p53, PMS2, MSH6, PTEN and beta-catenin. Molecular classification was established (POLE sequencing and p53, PMS2, MSH6 IHC).

Results: 73 viable PDX models were established, with an overall success rate of $78,5 \%$ ( $76,4 \%$ for $\mathrm{EEC}, 60 \%$ for $\mathrm{SC}, 80 \%$ for $\mathrm{CC}, 80 \%$ for MX, $100 \%$ for $\mathrm{UC}, 92,9 \%$ for $\mathrm{CS}$ ) Overall, xenograft tumors reproduced the pathological and IHC features of the primary tumors. The most significant differences were changes of histological grade in 4 EEC, and IHC differences in ER (4), p53 (5), and PTEN (4). Two EEC developed different phenotypes (SC and UC) in the PDX. Molecular classification was established in EEC cases (POLE 1, microsatellite unstable 10, microsatellite stable 7, and p53 mutant 1), and two cases changed to 553 mutant in the PDX. Mice with three PDX models were subjected to tyrosine kinase inhibition, with successful results in comparison with untreated mice.

Conclusions: PDX from EC patients reproduce the pathologic and IHCfeatures of human tumors. Our results confirm their utility in assessment of tumor progression and targeted therapy decisions.

1230 Mutation Analysis of Female Genital Tract Melanomas Uncovers Distinct Mutation Profiles

Ozlen Saglam, Jamie Teer, Yonghong Zhang, Tania Mesa, Sean Yoder, Jane Messina. Moffitt Cancer Center, Tampa, FL.

Background: There is little data about molecular alterations involved in initiation and progression of primary female urogenital tract melanoma (PFUTMM). Some variations in the mutation rates between tumors originating in the vulva and urogenital tract mucosa have been suggested in the literature.

Design: We analyzed 13 PFUTMM and 9 available normal tissue controls using the Agilent ClearSeq Comprehensive Cancer Panel and Illumina sequencing. Mutations were confirmed using precise somatic mutation detection in samples with a matched normal. The mutation rates of select genes were compared to those of cutaneous melanoma (MM) cases from the institutional tumor bank cohort $(\mathrm{n}=191)$.

Results: Mean age at diagnosis was 71 (range: 59-87). Primary anatomic sites included 5 vulva, 5 vagina, 1 urethra and 1 vulvovaginal; one case that recurred in the vulva was also tested. Tumor depth ranged from 0.42-11.6 mm (mean $3.61 \mathrm{~mm}$ ); eight cases had surface ulceration. Mean survival was 51.6 months. Mutation rates by genes in tumor samples were: 4 (31\%) KIT and ATRX, 3 (23\%) ALK and TP53, 2 (15\%) NF1, NRAS and RAF1, 1 BRAF. 


\section{Mutation Rates}

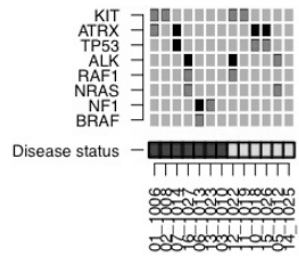

Green $=$ Vaginal $/$ mucosal; Blue $=$ Vulva

$\# 12 \& 16$ = Samples from same individual

Eight of 13 samples had $>1$ mutation. One of 2 cases with NF1 mutation had a unique spindle cell morphology; no other histomorphologic differences could be associated with mutation profile. NF1 and BRAF mutations were exclusively found in vulvar primaries. Mutation frequencies differed significantly between all PFUTMM and cutaneous MM for BRAF $(\mathrm{p}=0.0078)$ and $\mathrm{KIT}(\mathrm{p}=0.0071)$. ATRX mutation rate differed but was nonsignificant $(\mathrm{p}=0.067)$. The primary and matched recurrent tumors shared RAF1 and ALK mutations but had distinct drivers: KIT mutation was observed in the primary (vaginal) and NRAS in the recurrent tumor (vulvar).

Conclusions: In this small cohort, mutation frequencies between vaginal $/$ mucosal and vulvar samples were generally similar.

KIT and ATRX (truncating), ALK, and TP53 mutations are common in PFUTMM.

The BRAF (G466V) and NRAS (G12D, G13D) mutations observed are different than those generally observed in MM.

ATRX truncation mutations co-occur with TP53. However they are exclusive with KIT mutation.

The recurrent PFUTMM sample had a different driver mutation than the primary tumor Further exploration is needed to determine the extent of mutational differences between vaginal/mucosal and vulvar $\mathrm{MM}$

\section{Choriocarcinoma in Women: Analysis of a Case Series with} Genotyping

Johanna Savage, Kathleen M Murphy, Brigitte M Ronnett. Johns Hopkins Medical Institutions, Baltimore, MD; ProPath, Dallas, TX.

Background: Choriocarcinoma is an uncommon malignant neoplasm which can be gestational or nongestational. Distinction of these forms has prognostic and therapeutic implications. Data on the genetics of choriocarcinoma and the relationship to a concurrent gestation are limited.

Design: 19 tumors were genotyped using PCR amplification of 15 short tandem-repeat loci and the amelogenin locus (XY determination) (AmpF1STR Identifiler kit; Applied Biosystems, Foster City, CA). DNA patterns from tumor and maternal tissue, as well as villous tissue for those with a concurrent gestation, were compared to determine gestational versus non-gestational nature (containing versus lacking a paternal chromosome complement, respectively) and the relationship between the tumor and any concurrent gestation.

Results: Per genotyping, 17 tumors were gestational. These included 3 androgenetic homozygous XX uterine tumors without an accompanying placental component, 5 androgenetic homozygous XX uterine tumors with a concurrent genetically related complete hydatidiform mole (CHM), 1 androgenetic homozygous XX uterine cornual tumor separate from a genetically distinct second trimester placenta (dispermic twin gestation), 2 androgenetic homozygous XX ectopic fallopian tube tumors, 1 androgenetic homozygous XX ectopic ovarian tumor separate from a genetically distinct third trimester placenta (dispermic twin gestation), 3 biparental diploid (2 XX, $1 \mathrm{XY})$ intraplacental tumors genetically related to the placenta, 1 biparental diploid (XX) uterine tumor identified 4 weeks postpartum (placenta not available for analysis), and 1 biparental (XYY) uterine tumor with allelic imbalances (1 year after term delivery). Two tumors were non-gestational (both XX with allelic imbalances; 1 ovary, 1 pelvic). Conclusions: Gestational choriocarcinoma can be androgenetic or biparental. Most are androgenetic homozygous XX, including those with a genetically related concurrent CHM. Thus, homozygous XX CHMs are associated with some risk of significant gestational trophoblastic disease. Intraplacental choriocarcinomas are biparental and genetically related to the placenta. Eutopic or ectopic androgenetic choriocarcinoma separate from a concurrent intrauterine placenta is not derived from intraplacental tumor and is a form of dispermic twin gestation. Genotyping can determine whether choriocarcinoma in an ectopic site is gestational or non-gestational. While fallopian tube tumors are gestational, tumors in other sites can be non-gestational and should not be assumed to be derived from a regressed intrauterine gestational tumor.
1232

Prognostic Criteria in Vulvar and Vaginal Smooth Muscle Tumors: Evaluation of 68 Cases Comparing Multiple Classification Systems

Sadia Sayeed, Deyin Xing, Paul Weisman, Darya Buehler, Laura Warmke, Cora Uram-Tuculescu, Cherise Cortese, J Kenneth Schoolmeester. Mayo Clinic, Rochester, MN; Johns Hopkins Hospital, Baltimore, MD; University of Wisconsin, Madison, WI; Virginia Commonwealth University Health System, Richmond, VA; Mayo Clinic, Jacksonville, FL.

Background: While generally uncommon, smooth muscle tumors (SMT) of the vulva and vagina are the most frequent mesenchymal neoplasm of these organs, necessitating accurate classification of malignant potential. Three site specific classification systems have been proposed, 1 for vagina ${ }^{1}$ and 2 for vulva ${ }^{2,3}$, but it is our experience that many pathologists utilize 2014 WHO criteria intended for uterine SMTS. We collected a large cohort of cases with follow up to evaluate each classification system.

Design: In-house cases from multiple institutions were centrally reviewed for gross tumor size, degree of cytologic atypia, mitotic activity, presence of tumor cell and/ or ischemic necrosis, degree of cellularity, pattern of infiltration and cell phenotype (spindled, epithelioid). A total of 68 cases were collected consisting of 41 vaginal leiomyomata, 14 vulvar leiomyomata, 2 vulvar STUMP, 10 vaginal leiomyosarcomas (LMS), 2 vulvar LMS. Follow up was available for 55 patients ranging from 1 to 222 months (mean 74).

Results: Problematic aspects of the 1979 and 1996 classification systems are inclusion of microscopic assessment of infiltration and gross tumor size. Many tumors were either incidental findings or clinically suspected to be benign, resulting in enucleation or conservative excision, which precluded accurate assessment of infiltration in those cases and caused difficult in prognostic classification. Similarly, 3 otherwise conventional leiomyomata were upgraded to either SMT with increased risk of recurrence or low grade LMS due to infiltration and/or size. In lieu of gross tumor size and infiltration, tumor cell necrosis is included in the WHO uterine SMT classification, which serves as a more reliable criterion for malignant potential since it is not dependent on surgical procedure and is a frequent finding in our cases of LMS.

Conclusions: All systems accurately identify LMS. However, 1979 and 1996 criteria systems occasionally either preclude classification or upgrade otherwise conventional leiomyomata. The 2014 WHO criteria for uterine SMTs appear most accurate at classifying benign, intermediate and malignant SMTs of the vulva and vagina.

1.Obstet Gynecol. 1979;53(6):689-93.

2.Obstet Gynecol. 1979;53(2):213-7.

3.Am J Surg Pathol. 1996;20(7):779-93.

\section{$1233 \quad$ Abnormal p53 and p16 Staining Patterns Distinguish Uterine} Leiomyosarcoma from Inflammatory Myofibroblastic Tumor

Inga-Marie Schaefer, Jason L Hornick, Lynette M Sholl, Bradley J Quade, Marisa $R$ Nucci, Carlos Parra-Herran. Brigham and Women's Hospital/Harvard Medical School, Boston, MA; Sunnybrook Health Sciences Centre, University of Toronto, Toronto, ON, Canada.

Background: Uterine myxoid leiomyosarcoma is a rare but aggressive tumor with a relatively bland histologic appearance. Conventional and myxoid uterine leiomyosarcoma may be challenging to distinguish from inflammatory myofibroblastic tumor (IMT), which has a more indolent behavior and is amenable to targeted therapies. A significant subset of leiomyosarcomas harbor TP53 and/or CDKN2A genomic alterations. In this study, we examined the diagnostic value of p53 and p16 immunohistochemistry (IHC) in the distinction of uterine conventional and myxoid leiomyosarcoma from IMT, in correlation with targeted sequencing of TP53 and CDKN2A.

Design: We performed IHC using mouse monoclonal antibodies directed against p53 ( $1: 1200$ dilution; clone DO-1) and p16 (dilution 1:2; clone E6H4) in 49 tumors, including 23 uterine leiomyosarcomas (12 myxoid, absence of $A L K$ or ROS1 rearrangement confirmed by IHC, FISH and/or next generation sequencing; 11 conventional) and 26 IMT confirmed by IHC and/or next generation sequencing (12 uterine, 14 extrauterine). The complete coding regions of TP53 and CDKN2A were sequenced in 20 cases (4 myxoid and 11 conventional uterine leiomyosarcomas, 4 uterine and 1 extrauterine IMT). Results: Abnormal p53 staining patterns (strong and diffuse or null) were observed in 6/12 (50\%) myxoid and 6/11 (55\%) conventional uterine leiomyosarcomas; in contrast, all uterine and extrauterine IMT showed normal (wild-type) p 53 staining $(P<0.0001)$. Abnormal p53 patterns correlated with genomic mutation/deletion $(P=0.0001)$. Loss of p16 expression was detected in none of the uterine or extrauterine IMT, 5/10 (50\%) uterine myxoid leiomyosarcomas, and 2/11 (18\%) uterine conventional leiomyosarcomas $(P=0.0005)$. P16 loss correlated with $C D K N 2 A$ genomic deletion $(P=0.014)$, whereas strong and diffuse p16 staining, detected in $6 / 21(29 \%)$ leiomyosarcomas and $3 / 26(12 \%)$ IMT (all extrauterine), did not correlate with $C D K N 2 A$ alterations.

Conclusions: Abnormal p53 staining patterns and p 16 loss are observed in the majority of uterine leiomyosarcomas, showing high correlation with genomic alterations. In contrast, uterine and extrauterine IMT show normal p53 and p16 staining, highlighting the use of p53 and p16 IHC in the differential diagnosis of uterine mesenchymal neoplasms. 
1234

Clinicopathologic Analysis of MLH1-Hypermethylated Endometrial Carcinomas

Sheila Segura, Robert Soslow, Liying Zhang, Deborah Delair. Memorial Sloan Kettering Cancer Center, New York, NY.

Background: Approximately $20-25 \%$ of endometrial cancers (EMCA) show mismatch repair deficiency (MMR-D) but only a small subset is due to Lynch Syndrome. Most MMR-D EMCA are sporadic and show hypermethylation of the $M L H 1$ promoter. We sought to characterize the clinicopathologic features of these tumors.

Design: Clinical and pathologic data of $156 \mathrm{MLH1}$-hypermethylated EMCA from 156 patients were recorded including histotype, location of tumor, the presence of synchronous/metachronous tumors, patient age, stage at presentation, and clinical follow-up. The results of the MMR immunohistochemistry (IHC) were also noted. $M L H 1$ hypermethylation analysis was performed by pyrosequencing.

Results: Our series included 156 patients with a median age of diagnosis of 70 years (34-86) and a median follow up of 31 months ( 2 months - 15.5 years). MLHI hypermethylation analysis was performed in 146 primary EMCA and 10 metastatic lesions. Most cases showed endometrioid histology ( $\mathrm{n}=127)$ with $33.97 \%, 34.62 \%$, and $15.38 \%$ of these classified as FIGO grade 1, 2, and, 3 respectively. The remaining cases consisted of high grade endometrial carcinoma, NOS $(n=15)$, dedifferentiated undifferentiated carcinoma $(n=9)$, carcinosarcoma $(n=2)$ and mixed carcinomas $(n=3$; 2 endometrioid/clear cell and 1 clear cell/serous). Twenty-nine cases showed lower uterine segment involvement. By IHC, loss of MLH1 and PMS2 was identified in 146 cases, 8 cases showed equivocal/focally staining for MLH1 and isolated loss of PMS2 was seen in 2 cases. Synchronous ovarian carcinomas were seen in 5 patients (3 moderately differentiated endometrioid adenocarcinoma, 1 mixed undifferentiated/ high grade endometrioid, and 1 mixed clear/ endometrioid carcinoma). FIGO stage at presentation was as follows: I ( $n=111)$, II $(n=4)$, III $(n=30)$, IV $(n=11)$. At the time of follow-up, 8 patients $(5.12 \%$ ) died of disease (DOD), 122 (78.2\%) showed no evidence of disease (NED) while 26 (16.7\%) showed local recurrence and/or metastasis (AWD). Conclusions: The majority of MLH1-hypermethylated EMCA are low-grade endometrioid carcinomas (FIGO grades 1-2), however almost a third showed high grade histology. Patients were significantly older at the time of their EMCA diagnosis than the reported median age of patients with Lynch syndrome. In a small subset of cases (6.4\%), "false positive" immunoreactivity was observed for MLH1. A small percentage of patients $(3.2 \%)$ also presented with synchronous ovarian carcinomas. At the time of follow up, $22 \%$ of patients were either DOD or AWD. Detailed morphologic and outcome analyses are underway.

\section{Umbilical Lesions: A Clinicopathological Review}

Shenon Sethi, Smita Patel, Vijaya B Reddy, Pincas Bitterman, Ritu Ghai, Paolo Gattuso. Rush University Medical Center, Chicago, IL.

Background: Umbilical lesions are rare, and can be benign or malignant. The malignant lesions are almost always secondary (Sister Mary Joseph's nodule) and can either be a presenting sign of an undiagnosed primary or a sign of progression in a known patient. Published literature on this subject is sparse, and mainly exists as anecdotal case reports. This retrospective study was conducted to assess the epidemiological, clinical and histologic characteristics of umbilical lesions/masses.

Design: From our institution's pathology databases, the cases of umbilical lesions/ masses from January 1994 to August 2016 were retrieved and their clinicopathological features were reviewed.

Results: There were a total of 99 cases of umbilical lesions/masses with 78 females $(78.8 \%)$ and 21 males $(21.2 \%)$. Of these, 59 were malignant $(59.6 \%)$ and 40 benign $(40.4 \%)$. Among the malignant cases, 48 were females with a mean age of 65 years and 11 were males with a mean age of 66 years. All malignant lesions were secondary tumor deposits. 25 patients $(42.3 \%)$ had a metastatic tumor to the umbilicus with an average of 7 months from the original diagnosis (12 gynecological, 8 pancreas/gastrointestinal, 2 lymphomas and a case each of breast, prostate and melanoma). 19 patients (32.2\%) had synchronous metastasis ( 9 gynecological, 7 pancreas/gastrointestinal and 3 primary peritoneal tumors), in 15 patients $(25.4 \%)$ the umbilical mass was the first manifestation of an occult malignancy ( 8 gynecological, 4 pancreas/gastrointestinal), and in 3 patients no primary sites were identified on clinical follow-up. Out of the patients with a benign diagnosis, 30 were females $(75 \%)$ with a mean age of 52 years and 10 were males $(25 \%)$ with a mean age of 43 years. 8 patients $(2 \%)$ had a history of prior malignancy. The benign lesions included epidermal inclusion cysts (15), endometriosis (11), lipomas (3), neurofibromas (3), fibromas (3), abscesses (2), and a case each of fat necrosis, tubular apocrine adenoma, serous cystadenoma, and calcified nodule.

Conclusions: The majority of umbilical lesions/masses are seen in females $(78.8 \%)$. The most common metastatic tumor to the umbilicus is from the adjacent organs $(86.4 \%)$ with gynecologic system $(49.1 \%)$ as the most frequent primary followed by gastrointestinal tract $(32.2 \%)$. Umbilicus is a common location for endometriosis $(27.5 \%)$. There is no difference in the mean age among female and male patients for metastatic disease to the umbilicus. The age group for benign conditions in both males and females is a decade or more, younger than that for malignant lesions.

1236 Differential Expression of SOX17 in Type I and II Endometrial Carcinoma

Maryam Shahi, Mohamed El Hag, Hanqiao Zheng, Mahmoud Khalifa, Faqian Li. University of Minnesota, Minneapolis, MN; University of Pittsburgh Medical Center, Pittsburgh, PA.

Background: TCGA study reveals four distinct molecular subtypes of endometrial carcinoma (ECa). Traditional type II has p53 mutation and significant copy number increase in certain genes such as MYC, ERBB2, CCNE1, and SOX17. Traditional type I is further classified into ultramutated, hypermutated, and copy number low and is associated with SOX17 mutation at recurrent positions. The purpose of this study is to determine whether there is any difference in SOX17 expression between type I and II endometrial carcinoma and evaluate if SOX17 expression has any prognostic significance in ECa

Design: Hysterectomy specimens with ECa, diagnosed in 2010 in our institution were reviewed and p53, PR, SOX17 and MSI immunostains were performed on selected blocks. Percentage of SOX-17 and PR nuclear expression intensity (0-3) was estimated by two pathologists, independently. Morphologic feature including FIGO grade and nuclear grade (NG), immunomarker expression and 5 year survival rate were analyzed. Results: We found 79 pure endometrioid, 1 papillary serous carcinoma, 1 clear cell carcinoma and 7 mixed tumors. Expression of SOX17 and PR were inversely correlated with FIGO and nuclear grade (FIGO I vs. FIGO III, $\mathrm{p}<0.01$; 1 -way ANOVA) in pure endometrioid. MSI ECa had lower expression of SOX17 and PR, however only PR revealed a statistically significant difference ( $\mathrm{P} 0.08$ and $\mathrm{P}<0.05$ respectively; T-test) between MSI and MSS endometrioid carcinoma. Pure and combined serous carcinoma (with p53 overexpression) had a significantly higher SOX17 expression compared to NG3 endometrioid carcinoma with no serous component $(\mathrm{p}<0.05)$.

5 -years Survival analysis of 41 cases showed cases with higher SOX17 score had better survival probability than low SOX17 cases ( $73 \%$ vs. $55 \%)$ of endometrioid.

Conclusions: SOX17 shows a distinct pattern of expression in ECa. It is normally expressed in benign endometrial glands, retains in FIGO 1 (NG 1), but decreases in higher grade endometroid. However, serous carcinoma almost invariably shows overexpression of SOX17 that correlates with the overexpression of p53. The later finding is concordant with TCGA data that revealed SOX17 amplification in P53 mutated ECa.

1237 Malignant Transformation Arising in Mature Cystic Teratoma Palzum Sherpa, Reetu Baral, Trishna Kakshapati. Patan Academy of Health Sciences, Lalitpur, Nepal.

Background: The prognosis of patients with malignant transformation arising within mature cystic teratomas (MCT) is poor. However, preoperative diagnosis is difficult due to its rarity. The aim of this study was to evaluate the clinical and histopathological characteristics of such malignancies.

Design: A retrospective cross sectional study on a series of cases was performed in the Department of Pathology from April 2011 to March 2016. Data from the histopathology database were analyzed using SPSS version 16.0.

Results: MCT comprised $230(57.6 \%)$ out of total 399 cases of ovarian neoplams. Malignant transformation was noted in $7(3 \%)$ of the MCT.

\begin{tabular}{|c|c|c|c|c|c|c|c|}
\hline Cases & Age & $\begin{array}{l}\text { Primary } \\
\text { surgery }\end{array}$ & $\begin{array}{l}\text { Maximum } \\
\text { tumor } \\
\text { dimension } \\
(\mathrm{cm})\end{array}$ & $\begin{array}{l}\text { Tor- } \\
\text { sion }\end{array}$ & Diagnosis & $\begin{array}{l}\text { Organ (s) } \\
\text { involved }\end{array}$ & $\begin{array}{c}\text { TNM } \\
\text { staging }\end{array}$ \\
\hline 1 & 62 & $\begin{array}{l}\text { TAH with } \\
\text { BSO }\end{array}$ & 10 & $\begin{array}{l}\mathrm{Ab}- \\
\text { sent }\end{array}$ & $\begin{array}{l}\text { Squamous cell } \\
\text { carcinoma }\end{array}$ & $\begin{array}{l}\text { Left ovary } \\
\text { and omen- } \\
\text { tum }\end{array}$ & T3aNx \\
\hline 2 & 35 & $\begin{array}{l}\text { Salpingo- } \\
\text { oophorec- } \\
\text { tomy }\end{array}$ & 9 & $\begin{array}{l}\text { Pres- } \\
\text { ent }\end{array}$ & $\begin{array}{l}\text { Squamous cell } \\
\text { carcinoma }\end{array}$ & Ovary & T1aNx \\
\hline 3 & 70 & $\begin{array}{l}\text { TAH with } \\
\text { BSO }\end{array}$ & 14 & $\begin{array}{l}\mathrm{Ab}- \\
\text { sent }\end{array}$ & $\begin{array}{l}\text { Adenocarci- } \\
\text { noma }\end{array}$ & Left ovary & T1aNx \\
\hline 4 & 40 & $\begin{array}{c}\text { TAH with } \\
\text { BSO }\end{array}$ & 7 & $\begin{array}{l}\mathrm{Ab}- \\
\text { sent }\end{array}$ & $\begin{array}{l}\text { Squamous cell } \\
\text { carcinoma }\end{array}$ & Right ovary & TlaNx \\
\hline 5 & 47 & $\begin{array}{l}\text { TAH with } \\
\text { BSO }\end{array}$ & 8 & $\begin{array}{l}\mathrm{Ab}- \\
\text { sent }\end{array}$ & $\begin{array}{l}\text { Squamous cell } \\
\text { carcinoma }\end{array}$ & $\begin{array}{l}\text { Right ovary } \\
\text { and cervix }\end{array}$ & $\mathrm{T} 2 \mathrm{aNx}$ \\
\hline 6 & 50 & $\begin{array}{l}\text { TAH with } \\
\text { BSO }\end{array}$ & 12 & $\begin{array}{l}\mathrm{Ab}- \\
\text { sent }\end{array}$ & $\begin{array}{l}\text { Carcinoid } \\
\text { tumor }\end{array}$ & Left ovary & TlaNx \\
\hline 7 & 30 & $\begin{array}{l}\text { Salpingo- } \\
\text { oophorec- } \\
\text { tomy }\end{array}$ & 12 & $\begin{array}{l}\mathrm{Ab}- \\
\text { sent }\end{array}$ & $\begin{array}{l}\text { Squamous cell } \\
\text { carcinoma }\end{array}$ & $\begin{array}{l}\text { Ovary, fallo- } \\
\text { pian tube and } \\
\text { omentum }\end{array}$ & $\mathrm{T} 3 \mathrm{aNx}$ \\
\hline
\end{tabular}

The mean age at diagnosis of patients with malignant transformation of MCT was 47.57 years. The size of the tumor ranged from $7-14 \mathrm{~cm}$. The mean diameter of tumor was $10.28 \mathrm{~cm}$. There was significant difference in age between patients of MCT and malignant transformation arising within MCT. No significant association was noted in tumor size.

\begin{tabular}{|c|c|c|c|c|c|c|}
\hline & $\begin{array}{c}\text { MCT } \\
\text { Mean } \pm \text { SD }\end{array}$ & $\begin{array}{c}\text { MCT with } \\
\text { malignant } \\
\text { transformation } \\
\text { Mean } \pm \text { SD }\end{array}$ & $\begin{array}{c}\text { Mean } \\
\text { difference }\end{array}$ & $\begin{array}{c}95 \% \\
\text { confidence } \\
\text { interval } \\
\text { Lower }\end{array}$ & $\begin{array}{c}95 \% \\
\text { confidence } \\
\text { interval } \\
\text { Upper }\end{array}$ & P value \\
\hline Age & $32.59 \pm 11.9$ & $47.57 \pm 14.59$ & 14.98 & 5.90 & 24.05 & 0.001 \\
\hline $\begin{array}{c}\text { Tumor } \\
\text { size } \\
(\mathrm{cm})\end{array}$ & $8.2 \pm 3.53$ & $10.28 \pm 2.49$ & 2.08 & -0.57 & 4.74 & 0.124 \\
\hline
\end{tabular}

Torsion was noted in a single case. Squamous cell carcinoma was the commonest malignant tumor seen in 5 cases. The TNM stage distribution was T1aNx for four cases, $\mathrm{T} 2 \mathrm{aNx}$ for one case and T3aNx for two cases.

Conclusions: Squamous cell carcinoma is the most common malignancy arising in $\mathrm{MCT}$. Advanced age is a significant risk factor for malignant transformation in MCT. 
1238 Somatic Mutations in Cancer Driver Genes in Endometriosis in the Absence of Dysplasia or Cancer

Ie-Ming Shih, Ayse Ahan, Tian-Li Wang, Hiroshi Ogawa, Nicholas Papadopoulos. Johns Hopkins, Baltimore, MD; Seirei Mikatahara Hospital, Hamamatsu, Japan.

Background: Endometriosis is a relatively common disease, affecting up to $10 \%$ of women at reproductive ages. Although it is considered a "benign" disorder from both clinical and histopathologic perspectives, endometriosis may considerably compromise quality of life and cause significant morbidity including pelvic pain and infertility. It is characterized by normal-appearing ectopic endometrial tissues containing biphasic glandular and stromal components. The pathogenesis of endometriosis is intriguing- it has been considered as a benign inflammatory lesion, but shares features with malignant neoplasms such as invasion and dissemination. One of the most fundamental questions remains to be answered is to determine whether endometriosis, like cancer, harbors somatic sequence mutations.

Design: Whole exome sequencing was performed in 24 discrete endometriotic lesions. Selected mutations were then validated in micro-dissected glandular epithelium and surrounding stromal cells separately using targeted sequencing assays including SafeSeqS as well as immunohistochemistry.

Results: Exome sequencing detected somatic mutations in 20 (63\%) of 24 cases. Five (21\%) of them harbored at least one of the known cancer driver mutations involving ARID1A, PIK3CA, KRAS, and PPP2R1A of which the mutations are frequently detected in endometrioid carcinomas. The cancer driver gene mutations are unlikely a random process $(\mathrm{P}=0.0016$, binomial test) suggesting that they are selected during the development of endometriosis. Targeted analysis demonstrated PIK3CA and KRAS mutations in normal-appearing epithelial cells but not in stromal cells using SafeSeqS. ARID1A immunoreactivity was lost in the epithelium but retained in stromal in two cases showing ARID1A mutations.

Conclusions: We provide a new finding that benign endometriosis harbored somatic mutations including those found in human cancer. Most interestingly, the lesions had cancer driver mutations occurring in glandular epithelial cells but not in stromal cells, suggesting that the acquisition of mutation occurs early during epithelial differentiation in incipient endometriosis. Our unexpected result should have significant implications to study the pathogenesis of endometriosis and to lay the biological foundation for future molecular classification of endometriotic lesions.

1239 Squamous Cell Carcinoma and Precancerous Lesions of the Vulva; Histopathological and Immunohistochemical Analysis of 37 Cases Junyoung Shin, Eun Na Kim, Kyu-Rae Kim. University of Ulsan College of Medicine, Asan Medical Center, Seoul, Republic of Korea.

Background: Vulvar squamous cell carcinoma (VSCC) mostly develops through human papilloma virus (HPV)-related or p53-dependent pathways, but histologic diagnosis is often difficult especially in the latter. The objective of this study was to correlate the histopathological and immunohistochemical (IHC) expression of VSCC and the surrounding precursor lesions, and to detect diagnostic problems, if any.

Design: 37 surgically resected VSCC specimens at Asan Medical Center during 20052016, and 12 normal vulvar skin resected for nonneoplastic causes were included for the study. IHC stainings for $\mathrm{p} 16^{\mathrm{INK} 4 \mathrm{~A}}$ and $\mathrm{p} 53$ were performed. In five cases showing equivocal p53 expression, p53 DNA sequencing was performed after microdissection of the lesion to detect any mutations. Based on the IHC results and mutation study, strong continuous nuclear expression for p53 in basal layer with or without parabasal extension was only interpreted as a true positive.

Results: 36 of 37 (97\%) VSCCs showed VIN in adjacent skin, and all VSCC and VIN had the same IHC expression. 47\% showed P16 block (+) (probably HPV-related) and $42 \%$ showed P53 overexpression (p53-dependent). P16 block (+) and P53 overexpression were mutually exclusive in $86 \%$, but remaining cases were negative $(\mathrm{n}=4)$ or positive $(\mathrm{n}=1)$ for both antibodies. P16 block $(+)$ cases showed histologic features of usual VIN ( $\mathrm{uVIN}$ ) in all cases ( $\mathrm{n}=19)$, but P16(-) cases showed differentiated VIN (dVIN) in $65 \%$, and usual VIN (uVIN) in $29 \%$. $72 \%$ of dVIN showed strong P53 overexpression, but the remainings also had strong $\mathrm{p} 53$ expression in suprabasal layer only. Significant correlation was not identified between the differentiation keratinization status of VSCC and the causes. Lichen sclerosus was identified in $36 \%$ of P53 overexpressed dVIN. Severe nonspecific patch inflammation in the dermis was identified in both uVIN and dVIN. 14\% of P53 overexpressed lesions did not have appreciable cytologic abnormality in basal layer.

Conclusions: The causes of the squamous lesions could not always be predicted by histology alone since histopathologic features and immunohistochemical expression were correlated only in $73 \%$. It is important to set interpretation criteria of P53 overexpression. Further, histologic diagnosis of the adjacent skin or resection margin need to be further defined when they show strong p 53 overexpression without appreciable cytologic atypia (dVIN or not, and positive or negative margin).

\section{Mixed Epithelial and Non-Teratomatous Germ Cell Tumors of the Upper Gynecologic Tract}

Stephanie Skala, Andrew Sciallis. University of Michigan Health System, Ann Arbor, MI.

Background: Germ cell tumors such as yolk sac tumors are diagnosed much more commonly in young women than in women $>30$ years old. Previous reports suggest that the treatment and prognosis of these neoplasms are distinct between the two age groups, and that they may arise from different biologic pathways. Over the past few years, we have noted unusual cases of germ cell tumor associated with an epithelial component. We aimed to evaluate the clinicopathologic features of mixed epithelial and nonteratomatous germ cell tumors of the upper gynecologic tract in women $>30$ years old.
Design: We searched our institutional and consultative archives for non-teratomatous germ cell tumors in women $>30$ years old which yielded 8 cases. All available slides were re-reviewed, paying special attention to the presence of an epithelial component, and relevant clinicopathologic information was recorded.

Results: Of the 8 patients fulfilling our criteria, 6 had an ovarian lesion, 1 had an omental mass, and 1 presented with abnormal uterine bleeding in the context of a sonographically abnormal endometrium. Patient age at diagnosis ranged from 34-67 years.

In 3 patients ( 2 ovarian, 1 endometrial), there was an associated epithelial component that was seen in direct association with the germ cell tumor. The patient with the endometrial lesion was post-menopausal and curettage showed choriocarcinoma with a component of clear cell carcinoma. In the patients with ovarian masses (1 post-menopausal), both were yolk sac tumor (YST) arising in association with atypical endometriosis. Because of the presence of endometriosis, in 2 of the cases the germ cell component was not recognized as such and thought to be an endometrioid adenocarcinoma (ovarian YST and atypical endometriosis) or undifferentiated carcinoma (endometrial choriocarcinoma and clear cell carcinoma). In the 2 patients with YST and atypical endometriosis, 1 patient was FIGO stage IC while the other was at least stage IIC.

Conclusions: In our cohort, 3 of $8(38 \%)$ germ cell tumors in women $>30$ years old were associated with an epithelial component. Interestingly, both ovarian tumors showed YST and atypical endometriosis. These findings are in-line with previous reports positing a relationship between yolk sac tumor and epithelial neoplasia, particularly when presenting in women $>30-40$ years. The cases also highlight the potential for diagnostic misinterpretation. As mixed epithelial and germ cell tumors may not respond to typical germ cell tumor chemotherapy, documentation of an associated epithelial component may carry prognostic significance.

1241 PD-L1 Expression in Mismatch Repair-Deficient Endometrial Carcinomas, Including Lynch Syndrome-Associated and Sporadic MLH1 Promoter Hypermethylated Tumors

Emily A Sloan, Kari Ring, Brian C Willis, Susan C Modesitt, Anne Mills. University of Virginia, Charlottesville, VA.

Background: Mismatch repair (MMR)-deficient endometrial carcinomas (ECs) due to Lynch syndrome (LS)-associated germline mutations or sporadic $M L H 1$ promoter hypermethylation $(\mathrm{MLHIhm})$ are an immunogenic subset of ECs and may represent excellent candidates for immune-based therapies targeting the programmed death-1 (PD-1)/programmed death-ligand 1 (PD-L1) checkpoint pathway. In MMR-deficient colorectal carcinomas, early clinical trials have shown benefit from PD1 and PD-L1 inhibitors. This study evaluates PD-L1 expression in MMR-deficient ECs including LS-associated and $M L H 1 \mathrm{hm}$ cases, with comparison to MMR-intact ECs.

Design: Immunohistochemistry (IHC) for PD-L1/CD274 (SP142, Spring Bioscience) was performed on 38 MMR-deficient (20 LS-associated, $18 \mathrm{MLHI} \mathrm{hm}$ ) and 29 MMRintact ECs. Staining was scored in the tumor and the peritumoral immune compartment. Tumors were further characterized by proportion of cells stained. Immune staining was classified as minimal, moderate or brisk. MMR deficiency was defined as absence of tumor immunostaining for MLH1, PMS2, MSH2 and/or MSH6, and was performed at the time of original diagnosis as part of the institution's universal LS screening program. Results: The majority of MMR-deficient tumors were PD-L1 positive (53\%) in at least a subset of tumor cells, with predominantly focal (1-10\%) distribution. LS-associated tumors were more likely to be PD-L1 positive relative to $M L H 1 \mathrm{hm}$ tumors ( $70 \%$ vs. $33 \%, p=0.05)$. The MMR IHC pattern most uniformly associated with PD-L1 expression was MSH6 loss. Only 10\% of MMR-intact ECs demonstrated any tumoral PD-L1 expression at the $1-5 \%$ threshold; this was significantly lower than in MMR-deficient tumors $(p=0.0005)$. When reviewed by histologic grade, PD-L1 expression remained highest among LS-associated ECs followed by $M L H 1 \mathrm{hm}$ and MMR-intact carcinomas, respectively. Immune PD-L1 expression was seen in $100 \%$ of MMR-deficient and $66 \%$ of MMR-intact cases.

Conclusions: This study is the first to characterize differences in PD-L1 expression between LS-associated versus $M L H 1 \mathrm{hm}$ ECs. Tumoral PD-L1 expression was more common among the LS-associated cancers relative to $M L H I \mathrm{hm}$ and MMR-intact tumors, although sporadic cancers often showed PD-L1 positive immune stromal staining. These data suggest that MMR deficiency in EC may be a better predictor of response to PD-1/ PD-L1 inhibitor therapy than tumor grade, and potential benefit may vary based on the molecular mechanism of MMR defects.

\section{Targeted Genomic Profiling of Ovarian and Peritoneal Low-} Grade Serous Carcinomas (LGSC) with Clinicopathologic Correlation

$T$ Rinda Soong, Lynette M Sholl, Michele T Baltay, Bradley J Quade, Michelle S Hirsch, Christopher P Crum, Marisa R Nucci, Brooke E Howitt. Brigham and Women's Hospital, Boston, MA.

Background: LGSC are highly resistant to conventional chemotherapy. Little is known about targetable mutations in LGSC or molecular signatures that might distinguish ovarian from peritoneal LGSC. We characterized the molecular alterations of ovarian and peritoneal LGSC and evaluated their association with clinical features.

Design: Thirty-four LGSC from our institution were included. DNA from FFPE tissue was analyzed by a hybrid-capture next generation sequencing (NGS) assay that interrogates the full coding sequences of 309 genes for mutations and copy number variations (CNVs). Frequencies of molecular alterations and clinical features in subgroups were compared by either Mann-Whitney or Fisher's exact tests. Survival curves were compared with log-rank test.

Results: The study included ovarian $(\mathrm{n}=27)$ and peritoneal $(\mathrm{n}=7)$ LGSC with a median age of 56 yrs (range: $21-81 \mathrm{yrs}$ ). The majority presented at FIGO stage $3(43 \%)$ or 4 $(19 \%)$. A greater proportion of ovarian (vs peritoneal) LGSC presented with elevated CA125 (73\% vs 33\%) and were associated with serous borderline tumors $(56 \%$ vs $14 \%$ ). Median time to last clinical followup was 30 months (range: 8-225 months). 
There were 14 LGSC tumor recurrences and 2 related deaths, with an overall $5 \mathrm{yr}$ disease-free survival of $69 \%$. Advanced stage $(>3)$ showed 2 -fold increased hazard for recurrence with borderline statistical significance $(\mathrm{p}=0.06) . K R A S(32 \%), B R A F(10 \%)$, and NRAS $(5 \%)$ comprised the most common pathogenic mutations in this cohort. The most frequent chromosomal arm alterations included $1 \mathrm{p}$ loss $(35 \%), 22 \mathrm{q}$ loss $(22 \%)$ and 1q gain (19\%). A distinct subset of tumors (28\%) with no CNV was identified ("genetically stable"), of which a smaller proportion showed recurrence when compared with tumors having $\geq 10$ gene-level CNVs ( $13 \%$ vs $46 \%)$. No pathogenic alterations of TP53, PTEN, BRCA1, BRCA2, ESRI or PGR were detected. No significant association of molecular alteration with clinical or pathologic features was noted.

Conclusions: To our knowledge this is the largest series evaluating LGSC using a comprehensive targeted NGS panel. Results confirm the predominance of alterations in the RAS/RAF pathway and the relative lack of other pathogenic or targetable mutations. There are no significant differences in genetic alterations or recurrence risk between ovarian and peritoneal LGSC. Genetically stable LGSC may potentially be associated with a better prognosis.

\section{Focal Random-Appearing p16 Expression in the Cervix} Signifies HPV Infection Near the Squamo-Columnar Junction

T Rinda Soong, Jelena Mirkovic, Jan Brouwer, Natkrita Pohthipornthawat, Christopher P Crum, Michael Herfs. Brigham and Women's Hospital, Boston, MA; University of Liege, Liege, Belgium.

Background: Diffuse linear p16 immunoreactivity correlates with high-risk HPV+ squamous intraepithelial lesions (SILs) in the cervix. Little is known about the implications of random-appearing ("non-specific") expression of p16 in non-lesional epithelium. We addressed the hypothesis that focal random-appearing p16 expression signifies early HPV infection at or near the squamo-columnar junction (SCJ).

Design: Forty-two LEEP specimens from women with history of SIL but without histologic evidence of SIL were reviewed. The SCJ region was identified based on CK7 expression and morphologic features. Cases with visible SCJs $(n=24)$ were further evaluated by immunohistochemical staining with a p16 antibody as well as a E2 antibody, the latter a marker of early infection by HPV 16 (kindly provided by Y. Xue). Associations of clinicopathologic features with p16 and E2 expression were evaluated by Fisher's exact test.

Results: Median age was 34 yrs (range: $24-56$ yrs). Of the 24 specimens examined, $75 \%$ $(\mathrm{n}=18)$ showed focal positivity for $\mathrm{E} 2$, among which $78 \%$ also exhibited heterogeneous p16 expression that topographically correlated with E2 staining. Immunoreactivity for E2 was seen at and around the SCJ, with involvement of the metaplastic epithelium as well as adjacent or overlying endocervical columnar epithelium. A subset of these cases $(22 \%)$ displayed E2 expression that extended up to $2 \mathrm{~mm}$ into the neighboring ectocervix. All cases with p16 expression stained with E2. No significant association was seen between $\mathrm{p} 16 / \mathrm{E} 2$ positivity and clinicopathologic features including age, grade of prior SIL, history of recurrent SIL, smoking, parity or use of exogenous hormones. No biomarker staining was detected near margins. Median time to last clinical follow-up was 17 months (range: 6-38 months) and no recurrences were seen following excision in the cases examined.

Conclusions: Our data confirm that both normal-appearing SCJ and metaplastic cells can be a reservoir for HPV and that apparently non-specific or random-appearing p16 immunoreactivity in these cells reflects the presence of HPV nucleic acids. The distribution of E2 and p16 expression at and around the SCJ implies that risk of cervical SIL might depend on whether these infected cells are completely removed. Moreover, their distribution suggests that these infected cells could be ablated in advance by targeting the SCJ region, an approach that could be tested in high-risk HPV+ women who are vulnerable to subsequent SIL.

\section{International Endocervical Adenocarcinoma Criteria and}

\section{Classification (IECC)}

Simona Stolnicu, Iulia Barsan, Lien Hoang, Cristina Terinte, Anna Pesci, Sarit Aviel-Ronen, Takako Kiyokawa, Isabel Alvarado-Cabrero, Kay Park, Esther Oliva, Robert Soslow. University of Medicine and Pharmacy, Targu Mures, Mures, Romania; Vancouver General Hospital, Vancouver, Canada; Oncologic Institute, Iasi, Romania; Ospedale Sacro Coure Don Calabria, Negrar, Italy; Sheba Medical Center, TelHashomer, Israel; Jikei University School of Medicine, Tokyo, Japan; Hospital de Oncologia, Mexico City, Mexico; MSKCC, New York, NY; Massachusetts General Hospital, Massachusetts, MA.

Background: Our aim was to classify ECAs by using an iterative process based principally on morphological features linked to etiology (i.e. HPV infection) in contrast to the 2014 WHO classification.

Design: Full slide sets of 461 cases of endocervical adenocarcinoma were collected from 7 different institutions worldwide. WHO 2014 diagnoses were established using our interpretation of the vague definitions provided in the Blue Book. IECC was defined as follows: 1) Luminal mitoses and apoptosis=HPV-associated adenocarcinoma (HPVA); 2) No or limited HPVA features=NHPVA. HPVA were then subcategorized based on cytoplasmic features, mostly to provide continuity with pre-existing classification schemes (i.e. usual-type, adenosquamous, mucinous, etc), while NHPVA were subclassified based on published criteria defining morphologically, etiologically and phenotypically unified diagnostic entities (i.e. gastric-type, clear cell, etc). TMAs were constructed and immunohistochemistry for the following markers p16, p53, p63, Vimentin, PR, were performed on 253 cases. Block-like staining for p16 and overexpression of $\mathrm{p} 53$ ( $>75 \%$ tumor cell nuclei) were defined as positive. Endometrial and uterine adnexal adenocarcinomas involving cervix were excluded.

Results: The 5 most common WHO diagnoses were endometrioid ( $42 \%$; $76.2 \%$ p $16+$ ), mucinous NOS $(13 \% ; 80.6 \%$ p16+), usual-type $(11 \% ; 91.6 \%$ p16+), adenosquamous $(10 \% ; 75 \%$ p $16+)$, gastric-type $(9 \% ; 40.7 \%$ p $16+)$ while the 5 most common IECC diagnosis were usual-type $(67 \% ; 82.9 \% \mathrm{p} 16+)$, adenosquamous and gastric-type (both $19 \% ; 76.6$ and $40 \%$ p $16+$ respectively), clear cell and adenocarcinoma NOS (both $5 \%$; both $33.3 \%$ p16+). Results for other markers were tabulated.

Conclusions: The IECC generally reflects the distribution of ECAs diagnosed at large referral centers. True endometrioid carcinomas are extraordinarily rare when isthmic tumors are excluded. Immunohistochemical results support replacement of the WHO 2014 criteria with IECC.

1245 International Endocervical Criteria and Classification: Mucinous Endocervical Adenocarcinomas (MEA)

Simona Stolnicu, Iulia Barsan, Lien Hoang, Cristina Terinte, Anna Pesci, Sarit AvielRonen, Takako Kiyokawa, Isabel Alvarado-Cabrero, Kay Park, Esther Oliva, Robert Soslow. University of Medicine and Pharmacy, Targu Mures, Romania; Vancouver General Hospital, Vancouver, Canada; Oncologic Institute, Iasi, Romania; Ospedale Sacro Coure Don Calabria, Negrar, Italy; Sheba Medical Center, Tel-Hashomer, Israel; Jikei University School of Medicine, Tokyo, Japan; Hospital de Oncologia, Mexico City, Mexico; MSKCC, New York, NY; Massachusetts General Hospital, Massachusetts, MA. Background: Our aim was to classify MEAs using an iterative process based on morphological features linked to etiology (i.e. HPV infection) instead of the 2014 WHO classification, which is based on the type of mucinous differentiation.

Design: Full slide sets from 104 MEAs were collected from 7 different institutions worldwide. The "International Endocervical Criteria and Classification (IECC)" was defined as follows: 1) Luminal mitoses and apoptosis=HPV-associated adenocarcinoma (HPVA); 2) No or limited HPVA features=NHPVA. HPVA were then subcategorized based on cytoplasmic features, while tumors showing gastric-type differentiation lacking HPVA features were classified as gastric-type MEA regardless of the presence of goblet cells. Usual type and MEAs were distinguished by the extent of mucinous differentiation $(>50 \%)$. Immunohistochemistry was performed on TMAs for the following markers (p16, p53, HER2) on 41 cases.

Results: The distribution of WHO diagnoses was mucinous NOS (51\%), gastric-type $(37 \%)$ and intestinal (12\%), while the distribution of IECC diagnoses were gastric-type (64\%), mucinous and intestinal (both $35 \%$ ). Nearly all mucinous NOS and intestinal-type MECAs were p16 positive ( $80-100 \%)$, reflecting a high probability of HPV infection, unlike gastric-type MEAs (40\% p16+). P53 positivity was found in intestinal and gastric-type cases $(40 \%$ and $50 \%$ respectively). Only the latter carcinomas showed Her2 positivity (7\%).

Conclusions: Mucinous NOS and intestinal MEA of the HPVA type appear to be closely related tumors. Gastric-type adenocarcinomas lacking HPVA with or without intestinal differentiation are distinctive tumors. There are 2 possibilities that account for 16 positivity in gastric-type adenocarcinomas: HPV infection of pyloric precursor cells with GNAS mutation or cell cycle dysregulation.

1246 Subsequent Breast and High Grade Serous Carcinomas After Risk-Reducing Salpingo-Oophorectomy in BRCA Mutation Carriers and Patients with History of Breast Cancer

Melissa Straub, Mirna B Podoll, Stephanie N David, Stoudenmire J Charles, Georgia L Wiesner, Mohamed M Desouki. Vanderbilt, Nashville, TN; Vanderbilt-Ingram Cancer Center, Nashville, TN.

Background: Risk-reducing salpingo-oophorectomy (RRSO) is a procedure to reduce the risk of adnexal cancer in BRCA mutation carriers and for hormonal manipulation in women with breast cancer (BC). The goal of the study is to report the frequency of subsequent $\mathrm{BC}$ and high grade serous carcinoma (HGSC) following RRSO in BRCA 1 and 2 mutation carriers and in patients with personal history of $\mathrm{BC}$ with or without BRCA mutation.

Design: 147 consecutive patients who received a RRSO were reviewed. Patient's age, clinical history, BC histotype, gene mutation data, incidence of post RRSO BC and HGSC and time intervals were analyzed. RRSO cases with no BRCA mutation or history of BC were excluded.

Results: 147 cases were followed for a mean of 49 months (1-177 months). 97cases had a deleterious BRCA1 $(n=49)$ or BRCA2 $(n=48)$ mutation (Group 1) and 50 cases had no BRCA mutation and history of BC (Group 2). Prior to RRSO, 42 (43\%) cases in group 1 had a history of BC (invasive in 39 and DCIS in 3) and all cases in group 2 had a history of BC (invasive in 46 and DCIS in 4) (see diagram). There was no difference between the groups in the age at diagnosis for BC (Mean of 44 years). Gene testing for group 2 was either negative or not done $(n=44)$ or showed deleterious mutation in non-BRCA genes $(n=6)$. Following RRSO, $2 / 97$ cases $(2 \%)$ with BRCA1 mutation were found to have occult HGSC. There were also $1 \mathrm{BC}$ recurrence and 1 primary $\mathrm{BC}$ with BRCA1 mutation compared to 5 recurrent BC in Group $2(10 \%)$.

\begin{tabular}{|c|c|c|}
\hline $\begin{array}{c}\text { Group } 1 \\
\text { BRCA mutation } \\
\quad(n=97)\end{array}$ & & $\begin{array}{c}\text { Group } 2 \\
\text { No BRCA mutaion } \\
(n=50)\end{array}$ \\
\hline $\begin{array}{l}\text { History of BC } \\
(n=42,43 \%)\end{array}$ & $\begin{array}{c}\text { No history of BC } \\
(n=55,57 \%)\end{array}$ & $\begin{array}{l}\text { History of BC } \\
(n=50,100 \%)\end{array}$ \\
\hline \multicolumn{3}{|c|}{ Post Risk reducing salpingo-oophorectomy } \\
\hline $\begin{array}{c}\text { Met BC } \\
(1 / 42,2.4 \%) \\
48 \text { months after RRSO }\end{array}$ & $\begin{array}{c}\text { Primary BC } \\
(1 / 55,1.8 \%) \\
42 \text { months after RRSO }\end{array}$ & $\begin{array}{c}\text { Recurrent/met BC } \\
(5 / 50,10 \%)\end{array}$ \\
\hline $\begin{array}{c}\text { Occult HGSC } \\
\text { in both ovaries } \\
(1 / 42,2.4 \%)\end{array}$ & $\begin{array}{c}\text { Occult HGSC } \\
\text { in both tubes } \\
(1 / 55,1.8 \%)\end{array}$ & $\begin{array}{l}\text { HGSC } \\
(0)\end{array}$ \\
\hline
\end{tabular}


Conclusions: Our series of 147 cases found occult HGSC in 2/49 (4\%) BRCA1 mutation carriers and none in BRCA2 cases or non-BRCA BC cases. Further, no additional cases of HGSC were identified in a mean of 49 months of follow up. The risk of subsequent recurrent BC after RRSO appears to be higher $(10 \%)$ in patients with history of $\mathrm{BC}$ with no BRCA mutation when compared to (2\%) in BRCA mutation carriers. However, this difference may be due to the higher rate of risk reducing bilateral mastectomy in the BRCA group (not analyzed in this study). Interestingly, the 6 cases with history of $\mathrm{BC}$ and mutation in non BRCA genes have no $\mathrm{BC}$ recurrence.

1247 Mature Trophoblasts of the Maternal Interface Have a Low Proliferative Index in Placenta Creta

Kyle C Strickland, Jelena Mirkovic, Carlos Parra-Herran, Bradley J Quade, Daniela A Carusi. Brigham and Women's Hospital, Harvard Medical School, Boston, MA; University of Toronto and Sunnybrook Health Sciences Centre, Toronto, ON, Canada. Background: Based on treatment of early ectopic pregnancies, the anti-proliferative drug methotrexate has been used as a postpartum therapy for the conservative management of retained placenta accreta. However, there is no data from controlled studies to support its use, which prohibits breastfeeding and can have side. To understand the pathobiology of placenta accreta, we determined the proliferation index of mature placental trophoblasts from 3rd trimester placentas with a diagnosis of accreta, increta, or percreta.

Design: We searched our database (2005-2012) to identify hysterectomies for placenta accreta (PA), increta (PI), or percreta (PP). Additionally, placentas with microscopic $\mathrm{PA}$, retained products of conception (rPOCs) with PA, and placental site nodules (PSN) were studied. H\&E stains were reviewed to select blocks with the maximum number of trophoblasts at the maternal interface on a single slide. Cases with available material were evaluated by immunohistochemistry for CD146 (Mel-CAM) and MIB1. We evaluated the absolute number per slide (ps) and percentage of CD146/MIB-1 double-positive trophoblasts at the maternal interface. As controls, normal 3rd trimester placentas and placental site trophoblastic tumors (PSTT) were evaluated similarly. Results were compared using an unpaired, two-tailed Student's t-test.

Results: We evaluated 31 hysterectomies with placenta creta (10 PA, 10 PI, 11 PP), 10 cases of microscopic PA, 11 cases of retained POCs with PA, 10 PSN, 12 normal placentas, and 3 PSTT. CD146/MIB-1 double-positive cells were found in only $20 \%$ of PA (avg $=0.30 \mathrm{ps}$ ), $10 \%$ of PI (avg $=0.10 \mathrm{ps}$ ), and $27 \%$ of PP (avg $=0.45 \mathrm{ps})$ hysterectomy cases. Similar results were obtained for microscopic PA $(20 \%$, avg $=0.30 \mathrm{ps})$, rPOCs with PA $(18 \%$, avg $=0.18 \mathrm{ps})$, and PSN $(0 \%$, avg $=0.00 \mathrm{ps})$. In contrast, double-positive cells were found in $75 \%$ of normal placentas (avg $=1.25 \mathrm{ps}$ ) and in $100 \%$ of PSTT (avg $=51.67 \mathrm{ps)}$, which was greater than in all placenta creta hysterectomy specimens (creta vs. normal $\mathrm{p}=0.0005$ and creta vs. PSTT $\mathrm{p}<0.0001$ ). Conclusions: The pathobiology of morbidly adherent placenta is not based on active and continuing trophoblast proliferation. Neoplastic trophoblasts are the only entity for which targeting DNA synthesis has a rationale basis, and the use of cytotoxic or anti-proliferative medications for placenta creta is not supported by these findings.

1248 Evaluation of a Universal Mismatch Repair Protein Immunohistochemistry Screening Strategy in Women with Endometria Carcinoma 60 Years of Age or Younger

Sarah V Strickland, Barbara Norquist, Elizabeth Swisher, Mara H Rendi, Rochelle Garcia, Mark R Kilgore. University of Washington, Seattle, WA.

Background: Endometrial carcinoma (EC) is a manifestation of Lynch syndrome (LS), which is caused by mutations in DNA mismatch repair (MMR) genes. Immunohistochemical (IHC) expression of DNA MMR proteins MLH1, MSH2, MSH6, and PMS2 (MMR IHC) can be used to assess for potential LS. Prior to July 2013 MMR IHC for EC was individually requested without a uniform policy (selective screening). Beginning in July 2013 we screened all EC diagnosed $\leq 60$ years (universal screening). The goal of this study was to determine the impact of the universal MMR IHC screening strategy by comparing the frequency of LS evaluation/diagnosis in the 3 years prior to the MMR IHC policy to the 3 years following it.

Design: The medical record was searched for all cases of $\mathrm{EC}$ in women $\leq 60,7 / 1 / 10$ $7 / 31 / 16$ and MMR IHC results were recorded. In women with positive MMR IHC we assessed whether genetic testing was performed and genetic results.

Results: 268 women $\leq 60$ were diagnosed with EC during the study period. Universal MMR IHC screening resulted in more women being screened, $125 / 135$ (93\%) versus $53 / 133(40 \%)$ with selective screening, $\mathrm{p}<0.0001$. Universal screening resulted in more screen positive results, with $27 / 135(20 \%)$ screen positive in the universal group and $14 / 133(10.5 \%)$ in the selective group, $\mathrm{p}=0.04$. There were more cases with loss of MLH1/PMS2 in the universal group vs the selective group, $22 / 135(16.3 \%)$ vs. $8 / 133(6 \%) \mathrm{p}=0.01$, (table). Of the 41 screen positive women, only $19(46 \%)$ had documentation of genetic testing. Of those who underwent genetic testing, 5/7 (71\%) in the selective group had a mutation consistent with LS, vs. 3/12 (25\%) in the universal screening group, $\mathrm{p}=0.07$.

Conclusions: Universal MMR IHC screening of women $\leq 60$ detected more screen positive women than selective screening but did not result in a greater number of women with LS confirmed by genetic testing. An insufficient number of screen positive women had confirmatory genetic testing to be able to assess if this strategy will result in improved detection of LS. We are working on identifying and addressing the barriers to the genetic evaluation of LS following a positive IHC screen.

\begin{tabular}{|l|l|l|l|l|}
\hline EC in women $\leq 60$ & $\begin{array}{l}\text { Screen } \\
\text { positive } \\
(\%)\end{array}$ & $\begin{array}{l}\text { Loss of } \\
\text { MLH1+/- } \\
\text { PMS2N (\%) }\end{array}$ & $\begin{array}{l}\text { Loss of MSH2 } \\
\text { +-MSH6N } \\
(\%)\end{array}$ & $\begin{array}{l}\text { Confirmed LS by } \\
\text { genetic testingN } \\
(\%)\end{array}$ \\
\hline $\begin{array}{l}\text { Selectivescreening } \\
\mathrm{N}=133\end{array}$ & $14(10.5)$ & $8(6)$ & $6(4.5)$ & $5(3.8)$ \\
\hline $\begin{array}{l}\text { Universalscreening } \\
\mathrm{N}=135\end{array}$ & $27(20)$ & $22(16.3)$ & $5(3.7)$ & $3(2.3)$ \\
\hline
\end{tabular}

1249 Uterine Adenomatoid Tumor: A Neoplasm Having Frequent Association with Immunosuppressive Therapy

Daisuke Tamura, Daichi Maeda, Sultan A Halimi, Masato Okimura, Yukihiro Terada, Akiteru Goto. Akita University, Akita, Japan; Kabul Medical University, Kabul, Afghanistan.

Background: Adenomatoid tumor (AT) of the uterus is a benign tumor of presumed mesothelial origin. Although AT usually presents as a single intramural nodule, rare diffuse uterine ATs have been documented. Since some of these diffuse ATs have arisen in patients undergoing immunosuppressive therapy, an association between AT and immunosuppression has been suspected. However, no study has assessed the general correlation between the incidence of uterine AT and a patient's immunosuppressive status.

Design: We evaluated 369 hysterectomy specimens submitted to the Department of Pathology at the Akita University Hospital from 2012 to 2016 for the presence or absence of AT. Medical records of all patients were retrieved, which included their immunosuppressive status. Further, four autopsy cases of immunosuppressed females were examined for latent AT by in toto sectioning of the uterus.

Results: Of the 369 patients who underwent hysterectomy, 14 had been treated chronically with immunosuppressive agents such as predonisolone (PSL), Tacrolimus (FK506), cyclosporine (CyA), and azathioprine (AZA). Four ATs were identified in immunosuppressed patients $(4 / 15,27 \%)$, whereas only two non-immunosuppressed patients had ATs $(2 / 354: 0.57 \%)(\mathrm{p}<0.0001)$. In two of the immunosuppressed cases, AT was either multinodular or diffuse. Latent uterine AT was found in one of the four immunosuppressed female autopsy cases, which was composed of multiple nodules. ATs identified in this study are summarized in Table 1.

\begin{tabular}{|l|l|l|l|}
\hline & Age & Immunosuppresseive status & Types of AT \\
\hline Hysterectomy Case 1 & 38 & $\begin{array}{l}\text { +: PSL and AZA for systemic lupus } \\
\text { erithemotosus }\end{array}$ & $\begin{array}{l}\text { Multiple } \\
\text { nodules }\end{array}$ \\
\hline Hysterectomy Case 2 & 44 & $\begin{array}{l}\text { +: PSL and CyA for systemic lupus } \\
\text { erythematosus }\end{array}$ & Diffuse \\
\hline Hysterectomy Case 3 & 51 & +: PSL for rheumatoid arthritis & Single nodule \\
\hline Hysterectomy Case 4 & 52 & +: PSL and FK506 for glomerulonephritis & Single nodule \\
\hline Hysterectomy Case 5 & 48 & - & Single nodule \\
\hline Hysterectomy Case 6 & 60 & - & Single nodule \\
\hline Autopsy Case 1 & 55 & +: PSL and FK506 for MAGIC syndrome & $\begin{array}{l}\text { Multiple } \\
\text { nodules }\end{array}$ \\
\hline
\end{tabular}

Conclusions: The incidence of AT in immunosuppressed patients was significantly higher than in non-immunosuppressed patients. Diffuse or multinodular AT was found exclusively in immunosuppressed patients. Our data suggest that immunosuppression plays an important role in the tumorigenesis and progression of AT.

1250 Performance of Endometrial Sampling in Diagnosis of Endometrial Carcinoma

Catalin Taraboanta, Heidi Britton, Anna Plotkin, Nazila Azordegan, Philip B Clement, Blake Gilks. University of British Columbia, Vancouver, BC, Canada; University of Toronto, Mississauga, ON, Canada; University of Manitoba, Winnipeg, MB, Canada. Background: The last meta-analysis on endometrial biopsy accuracy concluded that a negative result is not very reassuring. The post-test probability for endometrial carcinoma after a negative result was $0.9 \%(95 \% \mathrm{CI}$ : $0.4 \%-2.4 \%)$. The combined miss rate for endometrial carcinoma and atypical hyperplasia was $11 \%$. Our study was conducted to determine if there have been any improvements over the 15 years. Design: All hysterectomies, with pathology reported between May 2011 and May 2015 , containing the words 'carcinoma', 'adenocarcinoma' or 'hyperplasia' in the final diagnosis, were reviewed. We excluded all primary ovarian, cervical, or fallopian tube carcinoma, metastatic carcinomas, outside consultation cases and cases with no previous endometrial sampling. The cases identified were then cross-referenced for negative endometrial sampling results reported up to five years prior to hysterectomy. We defined negative endometrial samples as either benign or insufficient/non-diagnostic, excluding cases of atypical hyperplasia and also those where follow-up sampling was recommended for any reason.

Results: We identified 172 cases with previous negative biopsies: 116 benign and 56 insufficient, out of 1,677 cases of hysterectomy for endometrial carcinoma. For the same period 22,875 negative biopsies were reported in our region.

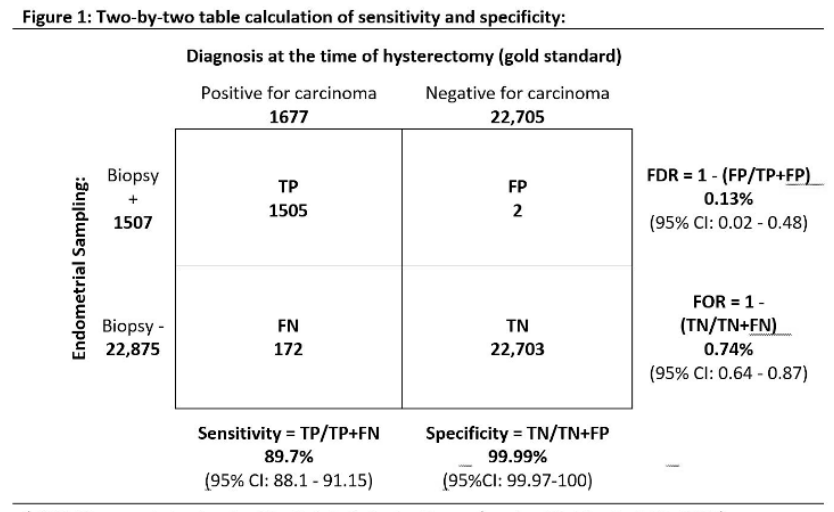

*95\% Cl generated using the MedCal statistical software (version 16.4.3 - April 26, 2016) 
The false negative/ miss rate was $10.2 \%$ for carcinoma or atypical hyperplasia. In a subset of 90 cases, the slides have been reviewed by two other pathologists with adjudication of the 12 cases where discrepancy was noted by a third pathologist, blinded to the study design and objective. The Kappa coefficient of agreement with the original diagnosis was excellent $\mathrm{k}=0.0832+/-0.053$. In 32 cases deeper levels were reviewed to increase diagnostic accuracy, to no additional benefit.

Conclusions: Our results demonstrate a significant lack of sensitivity of outpatient endometrial sampling in the diagnosis of endometrial malignancy/premalignancy. The sensitivity problems are a result of failure to sample abnormal endometrium. Independent review of slides or examination of additional levels are of no additional benefit.

1251 Sertoli-Leydig Cell Tumors: Examination of Morphologic Features and Correlation with Germline and Somatic DICER 1 Mutation Tatjana Terzic, Colin J Stewart, Leanne De Kock, William Foulkes, W Glenn McCluggage, Patricia Shaw, Blaise Clarke. University of Toronto, University Health Network, Toronto, ON, Canada; School for Women's and Infants' Health, University of Western Australia, Perth, WA, Australia; McGill University, Montreal, QC, Canada; Belfast Health and Social Care Trust, Royal Group of Hospitals Trust, Belfast, United Kingdom.

Background: Sertoli-Leydig cell tumors (SLCTs) are rare ovarian tumors. Up to $60 \%$ harbor somatic mutations in DICERI, but the prevalence of germline mutations is uncertain. We recently proposed that germline cases of SLCTs may be characterized by varied morphologic appearances such as juvenile granulosa cell tumor (JGCT)-like elements and heterologous differentiation. To date studies of hereditary and sporadic cases have been limited due to lack of germline data.

Design: The study cohort comprised 30 cases of SLCTs from patients (range 8-64 years old, average 27 years of age) for whom either the germline, somatic or both DICERI mutations were known. Slides were reviewed and histologic parameters (presence of bizarre nuclei, retiform and JGC-like morphology, heterologous elements) recorded by two pathologists, blinded to mutation status. The morphologic findings were then correlated with the germline and somatic status of DICERI mutation for each tumor patient.

Results: Fourteen patients had a germline DICERI mutation and 4 a somatic DICERI mutation only. Overall, 23 cases were DICERI associated (either germline or somatic) and 6 were DICER 1 independent. Six cases had heterologous elements, 4 displayed bizarre nuclei, 3 showed retiform morphology and 5 had JGC-like components. No association was found between any of the morphologic parameters and germline DICER 1 status. Hereditary cases did tend to occur at a younger age than sporadic cases DICERI associated cases. Patients that didn't have a DICERI mutation were older $(\mathrm{p}=0.0001)$ and had lower grade tumors $(\mathrm{p}=0.0041)$ compared to DICERI associated cases.

Conclusions: We present one of the largest studies to date correlating the morphologic and mutational characteristics of SLCTs. DICERI negative SLCTs present significantly more often with a lower tumoral grade and occur in older patients. However, no association could be established between the morphologic criteria and hereditary and sporadic DICERI associated cases. Hence, we suggest that all patients with SLCTs should undergo germline testing for DICERI.

1252 GATA3 in Müllerian Malignancies: A Potential Diagnostic Pitfall Tatjana Terzic, Sara Zadeh, Kristen Atkins, Krisztina Z Hanley, Anne Mills. University of Virginia, Charlottesville, VA; Emory University, Atlanta, GA.

Background: GATA3 immunohistochemistry is used as a marker of breast and urothelial differentiation, particularly in metastatic settings. However, its positivity has been described in tumors of gynecological tract (in one series up to $6 \%$ of ovarian serous carcinomas and $7 \%$ of endometrioid endometrial carcinomas and $33 \%$ of cervical squamous cell carcinomas), presenting a potential pitfall in the assignment of site-of-origin. However, data on the distribution of GATA3 expression in Müllerian malignancies is limited, particularly across different histologic subtypes.

Design: 100 ovarian carcinomas (OCA) $(20$ high-grade serous, 20 poorly differentiated, 20 clear cell, 20 mucinous, and 20 endometrioid) and 55 endometrial carcinomas (ECA) (10 low-grade endometrioid, 10 high-grade endometrioid, 15 serous, 10 MMMT, \& 10 clear cell), plus 9 cases of atypical hyperplasia were evaluated for immunohistochemical expression of GATA3 (L50-823) on tissue microarray ( 4 replicate $0.6 \mathrm{~cm}$ cores per case). 16 cervical squamous cell carcinomas presenting as metastases were also assessed on whole tissue sections. Staining was scored by $\%$ positive cells and intensity $(1-3+)$. Results: $8 \%$ of all ECA/hyperplasia were positive for GATA3: 2 serous carcinomas $(3+, 40 \%$ and $2+, 20 \%), 1$ MMMT $(2+, 5 \%)$ and 1 case of atypical hyperplasia $(1+$, $5 \%$ ). $6 \%$ of OCA were positive including 2 clear cell carcinomas $(2+, 30 \%$ and $1+$, $20 \%), 2$ mucinous adenocarcinomas $(3+, 50 \%$ and $3+, 20 \%), 1$ poorly differentiated carcinoma $(3+, 5 \%)$, and 1 endometrioid adenocarcinoma $(3+,>90 \%) .38 \%$ of metastatic cervical squamous cell carcinomas were positive ( $2+$ intensity in 1 and $1+$ in 5 cases) with nuclear positivity ranging from $30-50 \%$ of tumor cells.

Conclusions: GATA3 is positive in a subset of ECA and OCA including occasional cases with serous, clear cell, mucinous, MMMT, and endometrioid morphologies. While strong diffuse staining is rare in ovarian and endometrial primaries, it can occasionally be encountered and could present diagnostic difficulties if GATA3 is enlisted as a sole marker to assign mammary/urothelial origin. A larger proportion of cervical SCC strongly express GATA3 therefore particular caution should be exercised when using this stain to address pelvic tumors with a differential of cervical vs. bladder primary.
1253 Immunohistochemical Profiling of Mammary-Like Adenocarcinoma of the Vulva

Basile Tessier-Cloutier, Torsten Nielsen, Karama Asleh-Aburaya, Varsha I Shah, W Glenn McCluggage, Anna Tinker, C Blake Gilks. Vancouver General Hospital, Vancouver, BC, Canada; British Columbia Cancer Agency, Vancouver, BC, Canada; Singleton Hospital, Swansea, United Kingdom; Belfast Health and Social Care Trust, Belfast, United Kingdom.

Background: Mammary-like adenocarcinoma of the vulva is thought to be derived from embryologically ectopic breast tissue or mammary-like glands and has historically been treated in a similar way to breast cancer. We aimed to characterize a series of mammary-like adenocarcinomas of the vulva using an immunohistochemical (IHC) algorithm that identifies the major molecular subtypes of breast cancer, in an attempt to characterize the molecular events underlying this rare tumor type.

Design: 8 cases of mammary-like adenocarcinoma of the vulva from 5 different centres were collected. Each case was stained with an IHC panel known to have good concordance with gene expression assays for breast cancer intrinsic subtype. The panel included ER, PR, HER2, Ki-67, EGFR, CK5, nestin and INPP4B. We stained 4 cases of extramammary Paget disease of the vulva for comparison.

Results: The median age at diagnosis of the 8 patients with mammary-like adenocarcinoma was 71.7 years. All except one tumor was early stage (pT1N0). Based on our IHC panel, 5/8 (62\%) tumors were classified as luminal B, 2/8 (25\%) as human epidermal growth factor-2 (HER2) overexpressing and 1/8 (13\%) as luminal A. No tumors with a basal-like profile were identified (no tumor expressed nestin and all expressed INPP4B). ER was expressed in 6/8 (75\%) cases, PR in 3/8 (38\%), HER2 in $3 / 8(38 \%)$, EGFR in $1 / 8(13 \%)$, CK5 in $1 / 8(13 \%)$ and Ki- 67 was greater than $15 \%$ in $7 / 8(88 \%)$ cases. Of the 4 cases of Paget's disease of the vulva, $3(75 \%)$ were classified as luminal B and $1(25 \%)$ as luminal A based on our IHC panel.

Conclusions: Prognostication and management of mammary-like adenocarcinoma of the vulva is a challenging issue, mostly due to its rarity and the scarce information regarding the molecular pathology of these tumors. We show that current breast cancer subtyping can be applied to mammary-like vulvar adenocarcinomas; in this series of 8 cases, only luminal A, luminal B and HER2 subtypes were identified. The lack of basallike subtype may reflect the small sample size. A retrospective summary of treatment and clinical outcomes on this series is being compiled.

1254 Prognostic Implications of the Size, Number, and Location of Lymph Node Metastases in High-Grade Endometrial Carcinomas Lily Tran, Steven Maron, Ricardo R Lastra. University of Chicago, Chicago, IL. Background: In the United States, endometrial carcinoma represents the fourth most common malignancy in women. Twenty-one percent of cases are found to have regional metastases to lymph nodes and most commonly affect the pelvic and para-aortic lymph nodes. For uterine carcinomas, the TNM system characterizes involvement of the pelvic lymph nodes as $\mathrm{N} 1$ and para-aortic lymph nodes as $\mathrm{N} 2$, implying that the latter confers a worse prognosis. While staging in other organ systems, such as head and neck and breast, take into account the size and number of nodal involvement, this concept has not been explored for endometrial carcinomas. Furthermore, most of the current literature has mainly focused on the risk factors for nodal metastasis. Thus, this study is designed to investigate whether the size, number, and location of lymph node metastases in high-grade endometrial carcinomas have prognostic implications. Design: Patients with stage IIIC1 and IIIC2 high-grade endometrial carcinomas (i.e. endometrioid type FIGO grades 2 and 3, serous, and clear cell) between 2007 and 2014 were identified from our institutional pathology files. Characterization of the lymph node metastases, such as size ( $\leq 10 \mathrm{~mm}$ vs $>10 \mathrm{~mm}$ ), number of lymph nodes involved ( 1 vs $>1$ ), and location (pelvic vs para-aortic) were assessed. Outcomes measures included the duration of follow-up and length of time to recurrence.

Results: The study included 18 women with a mean age of 65 years. Among them, 6 were classified as high-grade endometrioid, 8 serous, and 4 clear cell. The mean followup period or time until recurrence was 20 months (range 3-82 months). One patient was lost to follow-up. Two-thirds of the patients had progressive or metastatic disease. The presence of $>1$ lymph node involvement was observed to have a statistically significant negative prognostic factor $(\mathrm{p}=0.021)$, whereas the size and location of the lymph node metastases were not statistically significant $(\mathrm{p}=0.376$ and $\mathrm{p}=0.624$, respectively).

Conclusions: Patients with $>1$ lymph node involvement due to high-grade endometrial carcinomas generally had a poorer prognosis. The size and location of the lymph node metastases did not predict a worse outcome. Due to our small sample size, further studies are needed to elucidate the correlation between the characteristics of the lymph node metastases and patient prognosis.

1255 Incidental Gynecological Tract Neoplasms in Women Undergoing Cystectomy for Urothelial Carcinoma

Lily Tran, Tatjana Antic, Ricardo R Lastra. University of Chicago, Chicago, IL.

Background: Urothelial carcinoma (UC) invasive into the muscularis propria of the urinary bladder is an indication for a cystectomy. For women, this may also include removal of gynecologic (GYN) organs, particularly if concerns of UC extension into the GYN tract exist. This study is aimed to assess the prevalence of incidental GYN neoplasms in cystectomy specimens.

Design: We identified 222 women who underwent an anterior pelvic exenteration for UC from 2004 to 2016. Pathology reports were reviewed to identify the incidence of unexpected GYN neoplasms.

Results: The mean age was 68 years (range 35-93). Concomitant secondary malignancies of the GYN tract were identified in 7 patients $(3 \%)$ which included endometrial adenocarcinoma $(\mathrm{n}=2,0.9 \%)$, high-grade squamous intraepithelial lesion $(\mathrm{n}=2,0.9 \%)$, endometrioid adenocarcinoma of the ovary $(\mathrm{n}=1,0.5 \%)$, and high-grade 
(HG) serous carcinoma of the ovary $(\mathrm{n}=1,0.5 \%)$ and fallopian tube $(\mathrm{n}=1,0.5 \%)$. Benign uterine neoplasms included adenomatoid tumor $(\mathrm{n}=2,0.9 \%)$ and adenomyoma $(\mathrm{n}=1$, $0.5 \%)$. Benign ovarian neoplasms included serous cystadenoma $(n=7,3 \%)$, serous cystadenofibroma $(\mathrm{n}=4,2 \%)$, benign Brenner tumor $(\mathrm{n}=5,2.3 \%)$, mature teratoma $(\mathrm{n}=4,2 \%)$, luteoma $(\mathrm{n}=2,0.9 \%)$, sex cord stromal tumor, $\mathrm{NOS}(\mathrm{n}=1,0.5 \%)$, mucinous cystadenoma $(\mathrm{n}=1,0.5 \%)$, endometrioid cystadenoma $(\mathrm{n}=1,0.5 \%)$, and thecoma $(\mathrm{n}=1$, $0.5 \%)$. Involvement of the GYN tract by UC was identified in 7 patients ( $3 \%$ ).

\begin{tabular}{|l|c|c|}
\hline Diagnosis & Number of Patients & Percentage \\
\hline Malignancies & & \\
\hline Endometrial adenocarcinoma & 2 & 0.9 \\
\hline High-grade squamous intraepithelial lesion & 2 & 0.9 \\
\hline Endometrioid adenocarcinoma of the ovary & 1 & 0.5 \\
\hline HG serous carcinoma of the ovary & 1 & 0.5 \\
\hline HG serous carcinoma of the fallopian tube & & 0.5 \\
\hline Benign uterine neoplasm & 2 & \\
\hline Adenomatoid tumor & 1 & 0.9 \\
\hline Adenomyoma & & 0.5 \\
\hline Benign ovarian neoplasms & 7 & \\
\hline Serous cystadenoma & 4 & 2 \\
\hline Serous cystadenofibroma & 5 & 2.3 \\
\hline Benign Brenner tumor & 4 & 2 \\
\hline Mature teratoma & 2 & 0.9 \\
\hline Luteoma & 1 & 0.5 \\
\hline Sex cord stromal tumor, NOS & 1 & 0.5 \\
\hline Mucinous cystadenoma & 1 & 0.5 \\
\hline Endometrioid cystadenoma & 1 & 0.5 \\
\hline Thecoma & 7 & 3 \\
\hline Involvement of GYN tract by UC & & \\
\hline
\end{tabular}

Conclusions: Involvement of the female reproductive organs by UC is a rare finding in anterior pelvic exenteration specimens. Coexisting neoplasms of the GYN tract are occasionally seen; therefore careful evaluation of these organs is vital.

1256 Utility of p16 vs. CK7 as a Predictive Biomarker in CIN 2

Brandon Umphress, Beatriz Sanchez, Ajit Paintal, Ritu Nayar, Kruti P Maniar. Northwestern University, Chicago, IL; IU Health Bloomington Hospital, Bloomington, IN.

Background: Cervical intraepithelial neoplasia grade 2 ( $\mathrm{CIN} 2)$ is an equivocal diagnosis requiring $\mathrm{p} 16$ immunohistochemical (IHC) positivity per current guidelines. We previously demonstrated that $\mathrm{p} 16(+)$ vs. p16(-) morphologic $-\mathrm{IN} 2$ is more often associated with subsequent -IN 3. Recently, biomarkers characteristic of squamocolumnar junction cells and positive in cervical high-grade intraepithelial lesions (HSIL) were described. Cervical low-grade squamous intraepithelial lesions (LSIL) expressing these markers (in particular CK7) are associated with a higher progression to HSIL. The utility of CK7 in morphologic CIN 2 is yet to be determined. Our objectives were to characterize the predictive value of CK7 positivity in CIN 2 for subsequent CIN 3 , and compare this to 16 in this setting.

Design: Cases diagnosed as CIN 2 and included in a previous study of p16 (Biocare, clone G175-405) were included. CK7 IHC (Dako, clone OV-TL12/30) was performed in all included cases. Follow-up information was previously obtained for all patients. The proportions of p16(+) (diffuse block staining), p16 (-) (all other patterns), CK 7(+) (diffuse, $\geq 5-6$ contiguous positive cells), and CK7(-) (all other patterns) cases were determined. The association of $\mathrm{CK} 7$ positivity and $\mathrm{p} 16$ positivity with subsequent $\mathrm{CIN}$ 3 was analyzed using Fisher exact test. The sensitivity/specificity of each biomarker as a predictor of CIN 3 in this setting was determined and compared using McNemar's test. All analyses were performed in IBM SPSS.

Results: 92 cases (from 90 patients) diagnosed as CIN 2 were included. $68 \%$ were positive for 16 and $91 \%$ were positive for CK7. 15/90 patients (16.7\%) had a subsequent diagnosis of CIN 3 on biopsy or excision, all of whom had preceding p16(+) and CK7(+) CIN 2 (sensitivity $100 \%$ for both markers). The specificity of p16 was $37.3 \%$ and that of CK7 was $8.9 \%$; these were significantly different $(p=<0.001)$. In independent contingency table analyses, $\mathrm{p} 16$ expression was significantly associated with subsequent CIN $3(p=0.004)$, while CK7 expression was not $(p=0.343)$.

Conclusions: CK 7 is expressed in a higher proportion of morphologic CIN 2 compared to 16 using these interpretation criteria. Positivity for either marker is highly sensitive for predicting subsequent CIN 3. However, CK7, unlike p16, is not significantly associated with the risk for subsequent $\mathrm{CIN} 3$, and p16 expression is more specific in this setting compared to CK7. Future studies in larger cohorts are needed to confirm these findings and determine the optimal staining/interpretation criteria for CK7.

1257 Clinicopathologic and Molecular Analysis of 25 Cases of Uterine Smooth Muscle Tumors of Uncertain Malignant Potential (STUMP)

Brandon Umphress, Qing Zhang, Jian-Jun Wei. Northwestern University Feinberg School of Medicine, Chicago, IL; Shandong University, Jinan, Shandong, China. Background: Smooth muscle tumors of uncertain malignant potential (STUMP) is a heterogeneous group of uterine tumors that is a diagnostic and prognostic dilemma. The clinicopathologic information available for this rare entity is insufficient in predicting potential recurrence or metastases. The objective of this study was to further characterize STUMP based on clinicopathologic and molecular properties.
Design: A total of 25 cases diagnosed as STUMP from Northwestern University and Qilu Hospital from 1995-2016 were reviewed. Cases were sub-classified using criteria delineated from Bell et al: atypical leiomyoma limited risk recurrence (ALM-LRR), atypical leiomyoma limited experience (ALM-LE), smooth muscle tumor low malignant potential (SMT-LMP), and mitotically active leiomyoma limited experience (MALLE). Tumors not fitting these criteria were classified as Other. Immunohistochemistry (IHC) for p16, p53, ER, PR, Ki-67 and gene sequencing analysis of p53, MED12, and PTEN was performed.

Results: Histologic analysis revealed 11 tumors classified as ALM-LRR (moderate/ severe atypia, mitoses 6-9, no necrosis), 4 tumors as ALM-LE (moderate/severe atypia, $<5$ mitosis, no necrosis), 4 tumors as MAL-LM (no cytologic atypia, mitoses $>15 / 10$ hpf), 2 tumors as SMT-LMP (coagulative necrosis and an infiltrating border), and 4 tumors as other (myxoid and epithelioid tumors, $<5$ mitoses). 19 cases had clinical follow-up data: 3 with recurrence ( 2 cases of ALM-LRR with metastatic lung disease and 1 case of ALM-LE with pelvic disease) while one patient died with disease from ALM-LRR. IHC and molecular findings are summarized below.

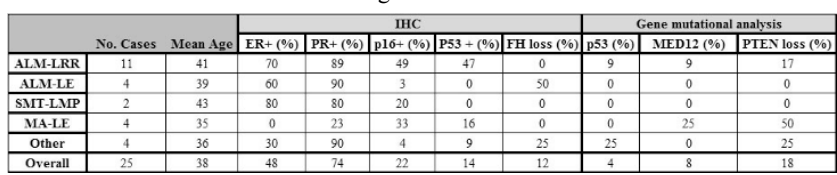

High ER and PR expression were seen in all groups $(>60 \%)$ with the exception of MAL-LE ( $<23 \%)$. Diffuse staining for p16 was seen in nearly $50 \%$ of ALM-LRR but much less common in other types. Molecular analysis revealed loss of PTEN in 50\% of MA-LE, $17 \%$ of ALM-LRR, and $25 \%$ of Other.

Conclusions: Our findings suggest that histologically defined sub-types of STUMP have varied clinicopathologic and molecular features. While most subtypes seem to have a benign clinical course, a small portion of ALM-LRR can result in recurrence.

\section{Clinical Utility of SATB2 in Determining Site of Origin in} Abdomino-Pelvic Tumors

Kavita Varma, Jing Yu, Binara Assylbekova, Xin Li, Esther Elishaev, Amal KanbourShakir, Beth Z Clark, David J Dabbs, Rohit Bhargava. Magee-Womens Hospital of UPMC, Pittsburgh, PA.

Background: Special AT-rich sequence binding protein 2 (SATB2) is a transcriptional regulator and play important role in osteoblastic and neuronal differentiation. SATB2 immunohistochemical (IHC) expression is selectively seen in colorectal epithelium, both benign and malignant. Limited data suggests lack of reactivity in female genital tract tumors. We hereby study the diagnostic value of SATB2 in determining site of origin when adenocarcinoma is present in the abdominopelvic region.

Design: SATB2 (clone EP281, Cell Marque, Rocklin CA) IHC expression was comprehensively examined in 460 primary tumors of various sites in the abdominopelvic region. The study included in-house tissue microarrays (TMAs) of primary ovarian epithelial tumors, endometrial, endocervical, and colonic carcinomas. TMA slides of gastric, pancreatic, small intestine, and rectal carcinomas were purchased from US Biomax (Rockville, MD). SATB2 nuclear staining was scored using an $\mathrm{H}$-score where the score ranges from 0 (negative) to 300 (diffuse strong positive). An H-score of 1 or higher was considered a positive result.

Results: SATB2 reactivity in different tumor types and extent of staining is shown in the table below.

\begin{tabular}{|l|l|l|}
\hline Tumor type & $\begin{array}{l}\text { Number +ve/Total; } \\
\text { Percentage }\end{array}$ & Mean H-score; Range \\
\hline Colonic adenocarcinoma & $23 / 30 ; 77 \%$ & $188 ; 1-300$ \\
\hline Endometrial tumors* & $7 / 43 ; 16 \%$ & $16 ; 1-70$ \\
\hline $\begin{array}{l}\text { Endocervical } \\
\text { adenocarcinoma }\end{array}$ & $1 / 19 ; 5 \%$ & $\begin{array}{l}1 \text { positive case with H score } \\
\text { of } 2\end{array}$ \\
\hline Ovarian tumors & $6 / 183 ; 3 \%$ & $26 ; 1-120$ \\
\hline Gastric adenocarcinoma & $2 / 34 ; 6 \%$ & $21 ; 1-40$ \\
\hline Pancreatic carcinoma & $3 / 78 ; 4 \%$ & $13 ; 10-20$ \\
\hline Rectal adenocarcinoma & $26 / 30 ; 87 \%$ & $102 ; 2-220$ \\
\hline Small bowel adenocarcinoma & $9 / 43 ; 21 \%$ & $55 ; 1-190$ \\
\hline
\end{tabular}

*3/31 endometrioid and $4 / 12$ non-endometrioid are positive; ${ }^{\wedge} 0 / 41$ borderline $(18$ mucinous, 4 seromucinous, 19 serous), 0/2 mucinous carcinomas, 0/5 high-grade carcinoma (unclassifiable or mixed), 1/66 serous, $3 / 40$ clear cell and 2/29 endometrioid are positive.

Conclusions: Colorectal carcinomas are often moderate to strongly positive for SATB2. Reactivity is significantly reduced in gastrointestinal tract tumors proximal to colon. Weak reactivity can be seen in gynecologic endometrioid and clear cell carcinomas. However, most mucinous tumors of gynecologic origin are negative for SATB2, which may be helpful in the differential diagnosis of mucinous tumor in the ovary.

1259 Application of a Pattern-Based Classification System of Endocervical Adenocarcinoma to Cervical Adenocarcinoma In Situ

Koah Vierkoetter, Teresa C Porter, David M Shimizu. John A. Burns School of Medicine, University of Hawaii, Honolulu, HI.

Background: A recently proposed pattern-based classification system for endocervical adenocarcinoma has demonstrated acceptable reproducibility and prognostic value. An area of relatively low diagnostic concordance remains the distinction between glandular lesions that exhibit non-destructive growth, adenocarcinoma in situ (AIS) and pattern A endocervical adenocarcinoma. 
Design: The current study retrospectively evaluated the volume and extent of disease in a series of 66 cases of AIS diagnosed in a cervical conization over 20 years at a single institution. Previously described histopathologic criteria defining pattern A endocervical adenocarcinoma were applied. Clinical, pathologic and temporal variables were collected for the excisions, hysterectomies and post-operative surveillance.

Results: The majority of cases demonstrated involvement by AIS in 2 or more quadrants $(62 \%)$. Average horizontal extent of disease was $4.1 \mathrm{~mm}$ (range $0.3-23 \mathrm{~mm}$ ). Vertical extent ranged from 0.2 to $8 \mathrm{~mm}$, with the majority of cases measuring $\leq 1 \mathrm{~mm}$. An average of $27 \%$ (range $4.5-94 \%$ ) of the total sections submitted were involved, with 8 cases displaying multicentric disease. Three of the 66 cases ( $4.5 \%$ ) previously diagnosed as AIS met diagnostic criteria for pattern classification A. Follow up data was available for 64 patients. The majority ( 37 patients) were managed conservatively, all of which were free of disease at last contact (average 66 months, range 3.3-237 months). Of the 27 patients who underwent hysterectomy, 20 cases demonstrated no residual disease, 6 showed residual AIS and 1 exhibited invasive adenocarcinoma.

Conclusions: These results support regarding AIS and non-destructive pattern A adenocarcinoma as a spectrum of disease. In the absence of destructive stromal invasion, tumors with these features may be considered along a diagnostic continuum, with similar clinical outcomes and management.

\section{The Role of Homer Scaffolding Protein 2 (HOMER2) in} Endometrial Cancer

Saloni Walia, Kate Lawrenson, Roxanne Manek, Paulette Mhawech-Fauceglia. University of Southern California, Los Angeles, CA; University of Southern California, Los Angeles, LA.

Background: In previous study we showed that HOMER2 (Homer scaffolding protein 2), a protein coding gene, was involved in low grade endometrioid adenocarcinoma (PLoS One 2015 Jul 1;10(7)). Besides its involvement in anchorage independent growth, there is little known about the function of HOMER2. The aim of this study is to evaluate HOMER 2 protein expression in a series of patients with endometrial adenocarcinoma (EC), and to determine its function in endometrial cell culture.

Design: The study was performed at a single institution and included a total of 336 cases of EC (type $\mathrm{I}=241$, type $\mathrm{II}=95$ ). Tumor microarray using 3 core punch biopsies on the 336 cases was constructed. HOMER 2 protein expression was detected by immunohistochemistry (IHC) using a polyclonal antibody. IHC was scored based on the intensity, and tumors were classified as negative $(0 / 1+)$ or positive $(2+/ 3+)$. Statistical analysis was used to evaluate the association of protein expression to the pathological parameters (histologic type, grade, stage, lymphovascular invasion, myometrial depth of invasion), and to the patients outcomes. Functional study using gene silencing and cell proliferation assay was done.

Results: HOMER2 protein showed significantly increased expression in type carcinomas compared to type II (67\% vs 30\%; p 0.0001). HOMER2 expression was associated with higher tumor grade $(\mathrm{p} 0.014)$. There was no association between HOMER2 expression and any other pathologic parameter. Lastly, patients with tumors expressing high HOMER2 protein levels had better outcomes (p 0.0012). Silencing gene expression using SiRNA showed decrease of HOMER 2 mRNA level by western blot (p 0.001), and an increase in proliferation rate (p 0.0049) after HOMER2 gene knockout in cell line.

Western Blot

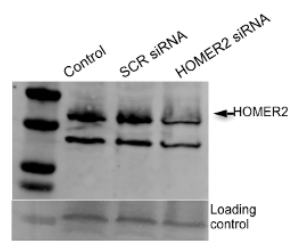

Proliferation Assay

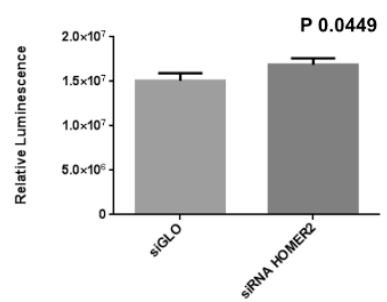

Conclusions: HOMER2 protein is overexpressed in type I endometrial adenocarcinomas It might be a promising predictor for better outcomes. The functional study shows that HOMER2 could play a role in inhibiting proliferation of tumor cells and hence, be a good tumor marker.

1261 Transcript-Based Classification of Omental Implants from Ovarian Borderline Serous Tumor Using a Cancer Gene Panel

Saloni Walia, Guisong Wang, Kate Lawrenson, GLarry Maxwell, Kathleen M Darcy, Iyare Izevbaye, Paulette Mhawech-Fauceglia. University of Southern California, Los Angeles, CA; Inova Fairfax Hospital, Falls Church, VA; Women's Health Integrated Resource Center, Annandale, VA; University of Alberta, Edmonton, AB, Canada.

Background: Generate a transcript-based classifier that stratifies morphologically invasive omental implants (IOI) from non-invasive omental implants(NIOI) vs omental metastatic carcinoma from ovarian serous cancer (MSC).

Design: The cases were retrieved form the archives were histologically classified as NIOI (n 10) or IOI (n 10). 4 cases of MSC were also included. The nCounter ${ }^{\circledR}$ GX Human Cancer Gene Reference Assay was used to profile 230 cancer genes and 6 control genes. Differentially expressed cancer genes(DEG) in MSC vs. NIOI were defined using t-test and NanoStringDiff packages. DEGs were used to cluster cases using the Eisen cluster 3.0 packages, and to develop a transcript-based classifier that predicts the probability of being MSC using Lasso in glmnet package. A risk score for classifying IOI as MSC or NIOI was generated using the algorithm in Figure 1 and coefficients from multivariate logistic modeling.

Results: There were 53 cancer-related genes that exhibited differential transcript expression in MSC compared with NIOI. Hierarchical clustering of these 53 genes stratified patients into two groups clustering IOI patients with NIOI or with MSC. A transcript-based classifier provided similar results to that obtained from the cluster analysis, predicting that $7 / 10$ of the IOI were very similar to NIOI, and $3 / 10$ were similar to MSC

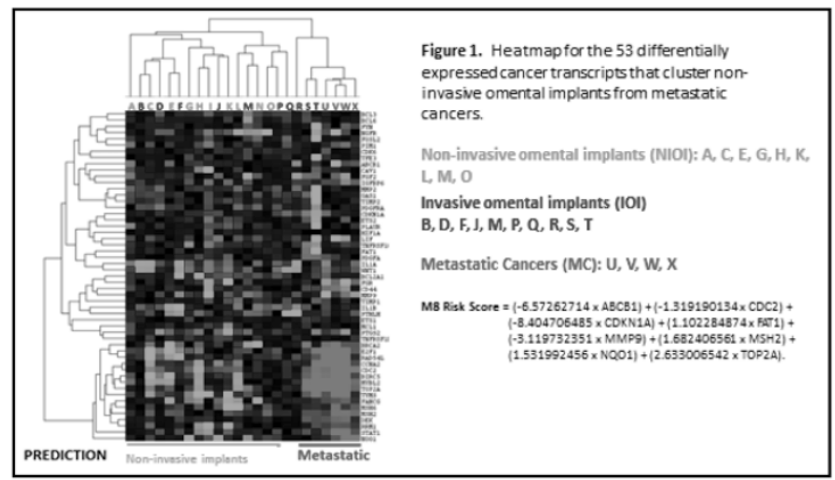

Conclusions: The M8 classifiers provided proof that differential transcript expression of a cancer gene panel was able to stratify cases of invasive implants into those that resembled a MSC vs. NIOI. Morphologic criteria are can't always differentiate the implants with clinical and therapeutic significance form benign ones. We suggest that in those cases with NIOI a molecular study using a panel of 8 DEG would be of great help. Additional research is needed to determine if these cancer genes are passengers or drivers in the malignant conversion of NIOI into invasive implants and metastatic cancer, further develop this classifier as a potential companion diagnostic

\section{Expression of Napsin A in Arias Stella Reaction: An Analysis} of 40 Cases

Ariel SY Wang, Ka Yu Tse, Oscar GW Wong, Annie NY Cheung, Philip PC Ip. The University of Hong Kong, Queen Mary Hospital, Hong Kong, SAR, Hong Kong. Background: In an established endometrial carcinoma, naspin A, in conjunction with a panel of other immunomarkers, is useful in the distinction of clear cell carcinoma from endometrioid carcinoma with clear cell change. Some investigators have recently addressed that napsin A can also be positive in benign mimics. In particular, Arias Stella reaction, one of the most the important differential diagnoses of clear cell carcinoma, has not been thoroughly investigated.

Design: Napsin A immunohistochemistry was performed on 34 endometrial and 6 cervical biopsies with Arias-Stella reaction. Any granular staining in the cell cytoplasm was considered positive. The proportion of glands stained by the marker was classified as negative, $<33 \%, 34-66 \%$ and $>66 \%$.

Results: 38 cases were gestational (miscarriages and $2^{\text {nd }}$ trimester abortions) and 2 were on progestin. Median age of patients was 36 years. 6 were endocervical polyps and 1 was an endometrial polyp, and the rest were endometrial biopsies. In each, the Arias Stella reaction was of the hypersecretory type. Nuclear atypia was accompanied either by strikingly clear cytoplasm or without cytoplasmic clearing. There were no mitotic figures. Prominent stromal predecidual change was found in only 12 cases $(30 \%)$. All $40(100 \%)$ were positive for napsin A and staining was readily identified under low-tomedium magnification. Positive staining involving $<33 \%$ and $34-66 \%$ of glands was found in $26(65 \%)$ and 13 cases $(32.5 \%)$, respectively. One $(2.5 \%)$ had $>66 \%$ staining. In any case that was positive, the positively stained cells involved part or most of a gland but never the entire gland. Staining involved only the cells with clear cytoplasm was seen in $22(55 \%)$, involved only the cells without clear cytoplasm was seen in 14 $(35 \%)$ or involved both types of cells was found in $4(10 \%)$. Follow-up was uneventful. Conclusions: In our series, naspin A was commonly positive in Arias Stella reaction, and particularly in cases without an accompanying prominent stromal predecidual change. The marker may stain cells with or without clear cytoplasm. Its use on small biopsies containing atypical clear cells, especially those without a known drug history or pregnancy status should be interpreted with caution.

1263 Tumoral and Peritumoral Immune PD-L1 Expression in Ovarian and Endometrial Clear Cell Carcinomas

Brian C Willis, Emily A Sloan, Anne Mills. University of Virginia, Charlottesville, VA. Background: Clear cell carcinoma represents a distinct histologic type of müllerian carcinoma that is uniquely resistant to conventional chemotherapy. Tumor and peritumoral immune cell expression of programmed death-ligand (PD-L1) has been associated with immune evasion in numerous tumor types and may be used to identify patients who will benefit from targeted immunotherapy. We evaluated PD-L1 expression in tumor cells and peritumoral immune cells in clear cell carcinomas of both ovarian and endometrial origin.

Design: Immunohistochemistry (IHC) for PD-L1 (SP142, Spring Bioscience) was performed on whole sections (WS) and tissue microarray (TMA) cores from 23 ovarian (13 WS, 10 TMA) and 23 endometrial (20 WS, 3 TMA). TMAs included 4 replicate 0.6 $\mathrm{mm}$ cores/case with sampling from different tumor areas. Membranous staining was scored by extent in tumor cells and the peritumoral immune compartment. 
Results: Tumor PD-L1 staining was seen in 35\% of ovarian tumors and $74 \%$ of endometrial tumors (Table 1). Extensive tumor staining $(>25 \%)$ was not seen in any ovarian tumors and was rarely identified in endometrial tumors $(13 \%)$. Two of the 3 endometrial tumors showing extensive staining were mismatch repair deficient as demonstrated by loss of MSH2 and MSH6 by IHC. Peritumoral immune PD-L1 staining was seen in $48 \%$ of ovarian and $74 \%$ of endometrial tumors. (Table 2) Considerable intratumoral heterogeneity of PD-L1 expression in tumor and immune cells was seen in both ovarian and endometrial primaries.

\begin{tabular}{|l|l|l|}
\hline Tumor Staining & Ovary & Endometrium \\
\hline 0 & $65 \%(15 / 23)$ & $26 \%(6 / 23)$ \\
\hline $1: 1-5 \%$ & $22 \%(5 / 23)$ & $9 \%(2 / 23)$ \\
\hline $2: 6-10 \%$ & $4 \%(1 / 23)$ & $30 \%(7 / 23)$ \\
\hline $3: 11-25 \%$ & $9 \%(2 / 23)$ & $22 \%(5 / 23)$ \\
\hline $4: 26-50 \%$ & $0 \%(0 / 23)$ & $9 \%(2 / 23)$ \\
\hline $5:>50 \%$ & $0 \%(0 / 23)$ & $4 \%(1 / 23)$ \\
\hline
\end{tabular}

\begin{tabular}{|l|l|l|}
\hline Immune Staining & Ovary & Endometrium \\
\hline $0: 0-5 \%$ & $57 \%(13 / 23)$ & $26 \%(6 / 23)$ \\
\hline $1: 5-10 \%$ & $26 \%(6 / 23)$ & $17 \%(4 / 23)$ \\
\hline $2: 10-50 \%$ & $22 \%(5 / 23)$ & $48 \%(11 / 23)$ \\
\hline $3:>50 \%$ & $0 \%(0 / 23)$ & $9 \%(2 / 23)$ \\
\hline
\end{tabular}

Conclusions: Müllerian clear cell carcinomas are enriched for tumoral and peritumoral immune PD-L1 expression, particularly endometrial primaries. These results suggest a potential benefit may be derived from immune checkpoint inhibitor therapy in a subse of these traditionally chemo-resistant tumors. Clinical trials are needed to establish staining thresholds required to predict response to PD-1/PD-L1 inhibitors; if extensive tumor staining is required, only a small subset of cases are likely to benefit. 1264 Pre-Operative Distinction of HGSC versus LGSC in Women at
Initial Presentation with Advanced Stage Pelvic Cancer

Rebecca J Wolsky, Matt A Price, Joseph Rabban. UCSF, San Francisco, CA.

Background: Distinction of high grade serous carcinoma (HGSC) versus low grade serous carcinoma (LGSC) in patients presenting with advanced stage pelvic cancer is important for initial management decisions since neoadjuvant chemotherapy may be considered for HGSC but not for LGSC. The 2014 WHO diagnostic criteria are based on whole slide sections and require assessment of nuclear atypia and mitotic index. Observer reproducibility is well established; p53 and p16 immunostaining can assist with equivocal cases. Whether these morphologic criteria can be applied in H\&Estained formalin-fixed cell blocks from aspiration/fluid specimens in patients at initial presentation with clinically advanced stage cancer has not been formally demonstrated but is vital for initial treatment planning.

Design: Ten pathologists reviewed H\&E stained slides from formalin-fixed cell blocks of aspiration/fluid specimens from 24 cases of advanced stage pelvic serous carcinoma (12 LGSC, 12 HGSC) without prior chemotherapy treatment. Observers were provided the 2014 WHO diagnostic criteria for LGSC and HGSC and example images. Observer were instructed that all cases were confirmed as pelvic serous carcinomas but they did not know the number of LGSC versus HGSC in the study nor did they know the p53, p16 immunostain results. Observers were instructed to classify each case as definitively HGSC, definitively LGSC or equivocal (if the morphologic features were not definitive for distinguishing HGSC versus LGSC).

Results: Overall interobserver agreement was moderate (Fleiss' kappa: 0.46). Among HGSC, agreement was $100 \%$ in $6 / 12$ cases; $90 \%$ in $2 / 12 ; 80 \%$ in $1 / 12 ; 60 \%$ in $1 / 12$. Among LGSC, agreement was $100 \%$ in $1 / 12$ cases; $90 \%$ in $2 / 12 ; 80 \%$ in $2 / 12 ; 70 \%$ in $1 / 12 ; 50 \%$ or less in $7 / 12$ cases. Four LGSC cases were classified as HGSC by more than half of observers. Equivocal diagnoses were rendered by 2 or more observers in $9 / 24$ cases, 8 of which were LGSC. Individual observer performance averaged $66 \%$ correct among all 24 cases (range $54 \%$ to $88 \%$ ). There was no statistical association between observer performance and practice profile.

Conclusions: Distinction of LGSC from HGSC in cell block preparations using morphologic features alone is challenging and may lead to sub-optimal management decisions, in particular due to misclassification of some LGSC as HGSC. Therefore, evaluation of cell block preparations from advanced staged pelvic serous carcinoma patients should routinely include p53 and p16 immunohistochemistry to confirm HGSC versus LGSC.

\section{Clinicopathological Features of Uterine Neuroendocrine} Tumors (NET)s

Adele Wong, Darrell Borger, Esther Oliva. Massachusetts General Hospital, Boston, MA; KK Women's and Children's Hospital, Singapore, Singapore.

Background: NETs of the uterine cervix (UCx) and corpus (UCor), classified as high grade neuroendocrine carcinomas (HGNEC) are rare. Characteristic findings of newer ancillary tests are poorly described.

Design: 10 NETs (5:UCx, 5:UCor) were evaluated for: age, amount of tumour necrosis, mitotic index, vascular invasion, stage and follow up. IHCs (MMR, p16, ki67) were performed on all tumors with $M L H 1$ methylation PCR test only on 1. Synaptophysin and chromogranin were done on all but $2 \mathrm{UCx}$ and EM on $2 \mathrm{UCx}$. Tumor from 5 NETs were macrodissected for DNA extraction from paraffin sections for NGS testing using a 91-gene panel, sequenced on the Illumina platform and analyzed with variant caller software.
Results: UCx patients were younger (41 vs 69.4). There were 9 HGNECs and 1 post adjuvant chemotherapy UCx low-grade NET, with pelvic LN metastases (pre-treatment features not assessed). 1 UCx had focal AIS and 1 UCor had a 70\% component of undifferentiated carcinoma. $3 \mathrm{UCx}$ and 3 UCor NETs had cells with modest cytoplasm and inconspicuous nuclear molding, where one of the $1 \mathrm{UCx}$ was a large cell HG NEC with prominent nucleoli. Mitotic index in UCor HG NECs was (9-121, mean 56) vs UCx (40-1, mean 20). All 9 HGNECs showed necrosis (all UCx and 3 UCor NETs with $10-30 \%$ necrosis; 2 UCor NETs with $>30-50 \%$ ). Ki67 in HGNECs was $>50 \%$. The UCx low-grade NET had 1 mitosis/10hpf and Ki67 of 1\% with no necrosis. All showed vascular invasion. Synaptophysin was uniformly stronger and relatively diffuse when compared to chromogranin. 1 UCx HGNEC required EM confirmation. All UCx NETs had block p16 positivity while variable staining (none, patchy, block positive) were seen in UCor NETs. All UCx NETs showed preserved MMR. 2 UCor NETs showed loss of $M L H 1 / P S M 2$. 1 UCor NET with focal loss of $M L H 1$ and rare cells staining for PSM2 was $M L H 1$ methylated. All NGS tested tumors (1 UCor, $4 \mathrm{UCx}$ ) had between 1 and 3 non-recurring mutations in PIK3CA, FGFR2, STAG2, POLE, BRCA, KIT, TP53, BRAF2 and FGFR3. Patients with UCor NETs had stage :II (2), IV (2) and 1 unstaged while patients with UCx NETs had stage IB2 (1), II (1), III (1) and IV (2). Positive pelvic LNs were recorded in 1 UCor and 4 UCx. At censure (follow up - UCor: 6-155, mean 71; UCx: 2-20, mean 12.8 months), 4 (2 UCor, 2 UCx) patients had died of disease and 2 (UCx) had distant metastases.

Conclusions: UCor NETs have variable p16 staining, can show $M L H 1 / P S M 2$ IHC loss due to $M L H 1$ methylation, and better survival compared to UCx NETs. UCs NETs are uniformly p 16 positive and MMR normal. Non-recurring somatic mutations were found in uterine NETs.

\section{TERRA Amplified (+) Primary Leiomyosarcomas (LMS) of the} Gynecologic (GYN)-Tract

Adele Wong, Kshitij S Arora, Zehra Ordulu, Vikram Deshpande, Esther Oliva. Massachusetts General Hospital, Boston, MA; KK Women's and Children's Hospital, Singapore, Singapore.

Background: TERRA+ infers alternative telomere lengthening (ALT) in malignant cells. Due to conflicting publications on abnormal ATRX in ALT+ uLMS, we assessed TERRA+ LMS in the GYN-tract: non-uterine (non-ut) and uterine corpus (u)LMS. Design: 53 uLMS and 6 non-utLMS ( 1 fallopian tube, 3 vagina, 2 vulva) were evaluated for: age, mitoses, LVI, stage and follow up. TERRA and GAPDH (housekeeping) RNA ISH (Affymetrix), and ATRX IHC (Sigma) were done on FFPE wholes sections $(n=8)$ and TMAs with two $3 \mathrm{~mm}$ cores from 51 cases. TERRA- $(\leq 10 \%$ tumor nuclei with $<3$ nuclear dots averaged over 2 cores) but GAPDH+, or ATRX- $(<1 \%$ tumour nuclei) on TMAs were repeated on whole sections, scored (-) if TERRA seen in $<5 \%$ tumor nuclei. ATRX was scored (-) if endothelial cells but not tumor stained. 10 uterine leiomyomas, leiomyomas with bizzare nuclei, cellular leiomyomas, epithelioid smooth muscle tumors and intravascular leiomyomatosis were tested on whole sections.

Results: Benign lesions had normal TERRA/ATRX results: TERRA-/ATRX+. 73.6\% uLMS and $66.7 \%$ non-utLMS were TERRA $+(75 \%$ had $>20 \%$ tumor nuclei each $>3$ dots).

\begin{tabular}{|l|c|c|}
\hline \multirow{2}{*}{ TERRA/ATRX } & \multicolumn{2}{|c|}{ Cases $(\mathrm{n}=59)$} \\
\cline { 2 - 3 } & $\begin{array}{c}\mathrm{uLMS} \\
\mathrm{n}=53(\%)\end{array}$ & $\begin{array}{c}\text { non-utLMS } \\
\mathrm{n}=6(\%)\end{array}$ \\
\hline TERRA+/ATRX+ & $22(41.5)$ & 0 \\
\hline TERRA+/ATRX- & $12(22.6)$ & $2(33.3)$ \\
\hline TERRA+/ATRX failed & $6(11.1)$ & $1(16.7)$ \\
\hline TERRA-/ATRX failed & $1(1.9)$ & 0 \\
\hline TERRA-/ATRX+ & $12(22.6)$ & $3(50)$ \\
\hline
\end{tabular}

Neither TERRA nor ATRX status were associated with specific morphology (spindle, epithelioid, myxoid, mixed) or age differences (uLMS: 56.6 vs 59.9, non-utLMS: 46 vs 42.3). Mitotic index was not significantly higher in TERRA+ uLMS (uLMS TERRA+:2-43, mean 17.8 vs TERRA-:2-64, mean 13.6; non-utLMS TERRA+:2-31, mean 16.3 vs TERRA-:1-8, mean 4.3 ). LVI was not significantly more common in TERRA+ uLMS (31.6\% vs $18.1 \%)$. Patients with TERRA+ uLMS were stage IA:2, IB:17, II:1, III:1, IV:15 and TERRA-: IA:3, IB:3, II:1, IV:6. Follow-up (months, n=56) was 7-255, mean 43.8 (TERRA+ uLMS) vs 4-143, mean 61 (TERRA-), and 7-37, mean 24 (TERRA+ non-utLMS) vs 29-92, mean 58 (TERRA-). 43.5\% uLMS and $66.7 \%$ non-utLMS TERRA+ patients died of disease; $54.5 \%$ with uLMS alive had metastases. $50 \%$ uLMS and $33.3 \%$ non-utLMS TERRA- patients died of disease; $60 \%$ with uLMS alive had metastases.

Conclusions: LMS in the GYN-tract are commonly TERRA+ with non-utLMS almost always showing abnormal ATRX-. Contrary to a recent report, only a subset of uLMS deaths were TERRA+/ALT + and fewer still were ATRX-, even among Stage I patients. Higher mitotic index and LVI were not significantly more common in TERRA+ uLMS.

1267 Intraoperative Evaluation of Prophylactic Hysterectomy and Salpingo-Oophorectomy Specimens in Hereditary Gynecologic Cancer Syndromes

Serena Wong, Natalia Buza. Yale School of Medicine, New Haven, CT.

Background: Prophylactic total hysterectomy (TH) and bilateral salpingooophorectomy (BSO) are increasingly common procedures in women at genetic risk for gynecologic malignancies. Intraoperative pathology diagnosis of malignancy provides the opportunity for immediate surgical staging and helps avoiding a second surgery. However, no standard guidelines exist for optimal intraoperative evaluation (IOE) of these specimens. 
Design: We performed a retrospective analysis of prophylactic $\mathrm{TH}$ and $\mathrm{BSO}$ cases to assess the presence of gross findings, frozen and permanent section sampling practices, frozen section diagnoses and diagnostic discrepancies. All prophylactic TH and BSO cases between 1990-2016 were retrieved from our departmental archives. All slides with malignancies were reviewed.

Results: A total of 254 cases were included in the study: 21 with Lynch syndrome (LS) , 190 with BRCA $1 / 2$, and 143 cases with strong family or personal history (non-Lynch, non-BRCA). Eleven of $21(52 \%)$ LS cases, 78 of $190(41 \%)$ BRCA cases and 46 of $143(32 \%)$ non-BRCA, non-Lynch cases were sent for IOE. Twelve patients had a final diagnosis of malignancy (invasive or in situ) on permanent sections. Only 1 of these 12 cases was sent for IOE, but the tumor was not sampled for frozen section due to lack of a gross lesion. Four of the 12 malignant cases (33.3\%) had a corresponding gross lesion.

\begin{tabular}{|l|l|l|l|l|l|l|}
\hline $\begin{array}{l}\text { Cancer } \\
\text { syndrome } \\
\mathrm{n}\end{array}$ & $\begin{array}{l}\text { Sent } \\
\text { for } \\
\text { IOE } \\
\mathrm{n}(\%)\end{array}$ & $\begin{array}{l}\text { Significant } \\
\text { gross } \\
\text { intraop. } \\
\text { findings } \\
\mathrm{n} / \mathrm{n}(\%)\end{array}$ & $\begin{array}{l}\text { Frozen } \\
\text { sections } \\
\text { submitted } \\
\mathrm{n} / \mathrm{n}(\%)\end{array}$ & $\begin{array}{l}\text { Malignancy } \\
\text { on frozen } \\
\text { sections } \\
\mathrm{n}\end{array}$ & $\begin{array}{l}\text { Malignancy } \\
\text { on permanent } \\
\text { sections } \\
\mathrm{n} / \mathrm{n}(\%)\end{array}$ & $\begin{array}{l}\text { Frozen/ } \\
\text { permanent } \\
\text { discrepancy } \\
\mathrm{n} / \mathrm{n}(\%)\end{array}$ \\
\hline $\begin{array}{l}\text { Lynch } \\
\mathrm{n}=21\end{array}$ & $\begin{array}{l}11 \\
(52 \%)\end{array}$ & $6 / 11(55 \%)$ & $\begin{array}{l}10 / 11 \\
(91 \%)\end{array}$ & 0 & $\begin{array}{l}1 / 21(4.8 \%) \\
(1 \text { endometrial } \\
\text { adenocarcinoma) }\end{array}$ & $0(0 \%)$ \\
\hline $\begin{array}{l}\text { BRCA } 1 / 2 \\
\mathrm{n}=190\end{array}$ & $\begin{array}{l}78 \\
(41 \%)\end{array}$ & $\begin{array}{l}43 / 78 \\
(55 \%)\end{array}$ & $\begin{array}{l}68 / 78 \\
(87 \%)\end{array}$ & 0 & $\begin{array}{l}9 / 190(4.7 \%) \\
(6 \text { tubal/ } \\
\text { ovarian high } \\
\text { grade serous } \\
\text { carcinomas, } \\
\text { STIC, } 1 \text { adult } \\
\text { granulosa cell } \\
\text { tumor) }\end{array}$ & $\begin{array}{l}1 / 78 \\
(1.3 \%)\end{array}$ \\
\hline $\begin{array}{l}\text { Non- } \\
\text { Lynch/ } \\
\text { Non- } \\
\begin{array}{l}\text { BRCA } \\
\mathrm{n}=143\end{array}\end{array}$ & $\begin{array}{l}46 \\
(32 \%)\end{array}$ & $\begin{array}{l}26 / 46 \\
(57 \%)\end{array}$ & $\begin{array}{l}41 / 46 \\
(89 \%)\end{array}$ & 0 & $\begin{array}{l}2 / 143(1.4 \%) \\
(2 \text { metastatic } \\
\text { breast } \\
\text { carcinomas })\end{array}$ & $0(0 \%)$ \\
\hline
\end{tabular}

Conclusions: Only less than half of all cases were sent for IOE, with LS cases having the highest proportion $(52 \%)$ among the 3 groups. The incidence of malignancy combining all 3 groups was $4.7 \%$. Among all cases with malignancy, one third had a corresponding gross lesion and could have been diagnosed on frozen section had they been sent for IOE. All TH and BSO specimens from patients with a known or suspected hereditary gynecologic cancer syndrome should be sent for IOE and significant gross lesions should be sampled for frozen section.

1268 Mitotically Active Uterine Leiomyomata Exhibit Similar Clinical Outcomes to Conventional Leiomyomata: A Clinicopathologic Study with Long-Term Follow-Up

Elizabeth Yiru Wu, Yin (Rex) Hung, Marisa R Nucci, Bradley J Quade. Brigham and Women's Hospital, Boston, MA.

Background: Accurate histologic distinction between benign and malignant uterine smooth muscle neoplasms is crucial given disparate clinical outcomes. Mitotically active uterine leiomyomata, defined as at least 5 mitoses (mits) per $10 \mathrm{high}$ power fields (hpfs) in the absence of tumoral necrosis or significant cellular atypia, are believed to be clinically indolent. Nevertheless, long-term follow-up data remain lacking.

Design: This study included 49 patients with mitotically active leiomyomata diagnosed at our institution between 1983-2014 with at least 2 years of follow-up (24 myomectomies, 22 hysterectomies, 1 endometrial curettage, and 2 extrauterine excisions). We included a control group of 53 conventional leiomyomata, with $<5$ mits per $10 \mathrm{hpfs}$ and no geographic necrosis or atypia. We obtained clinicopathologic data and evaluated long-term outcomes.

Results: The age of presentation for mitotically active leiomyomata was 25-57 (median 39 ) $\mathrm{yr}$, similar to the control group (median $43 \mathrm{yr} ; p=0.20$ ). Follow-up for patients with mitotically active leiomyomata and controls were 2.0-26.7 (median 11.7) yr and 2.0-33.7 (median 14.1) yr, respectively. Our cohort of mitotically active leiomyomata had 5-20 mitoses (median 6) per $10 \mathrm{hpfs}$. We found no significant difference in the re-operation rate after myomectomy between mitotically active leiomyomata $(13 / 49=27 \%)$ and controls $(8 / 53=15 \% ; p=0.22)$. Mitotically active leiomyomata requiring re-operation did so at 2-22 years, and had no significant difference in re-operation rate between those with 10 or more mits $(4 / 7=57 \%)$ and those with $5-9$ mits $(9 / 20=45 \% ; p=0.68)$. No cases recurred after hysterectomy; no patients developed metastases or died of tumor. Mitotic rates correlated with weights of myomectomy specimens (Spearman's coefficient $=0.69$ ). Re-operation rates in mitotically active leiomyomata were not influenced by the presence of mild atypia $(1 / 3=33 \%)$ or ischemic necrosis $(3 / 9=33 \%)$. No correlation was seen between maximal mitotic count and the phase (proliferative, secretary, or inactive) of the endometrium (Spearman's coefficient $=0.24$ ).

Conclusions: In this study with a median follow-up of $12.1 \mathrm{yr}$, mitotically active and conventional leiomyomata exhibit similar clinical outcome and re-operation rates. Higher mitotic rate also does not alter risk within mitotically active leiomyomata. Reoperation rates are not affected by the presence of mild atypia or ischemic necrosis. Thus, increased mitotic activity in leiomyomata is not predictive of adverse long-term outcomes.
1269 Prior HC2 HPV Testing Results of 2546 Women with Histologically Diagnosed Cervical Intraepithelial Neoplasia 2/3

Tao Wu, Christopher Griffith, BaoWen Zheng, Xiangdong Ding, Zhengyu Zeng, Yaoming Liang, Chengquan Zhao. Guangzhou Kingmed Diagnostics, Guangzhou, China; Emory University, Atlanta, GA; University of Pittsburgh, Pittsburgh, PA.

Background: High grade squamous lesions caused by persistent high risk Human Papillomavirus (hrHPV) infection are regarded as precursors to cervical cancer. The aim of this study is to evaluate hrHPV testing and Pap results preceding histologic diagnoses of CIN $2 / 3$ in China's largest CAP certified clinical laboratory.

Design: A search was performed to identify all cases of CIN $2 / 3$ histologically diagnosed from January 2011 to August 2016 and cases with prior HC2 HPV testing within the preceding 6 months before histological diagnosis were included in this study.

Results: 2546 patients with histologically diagnosed $\mathrm{CIN} 2 / 3$ had prior $\mathrm{HC} 2$ testing results within the prior 6 months. The average age was 38.5 years (range 18-77 years). The average interval between hrHPV testing and histologic diagnosis was 1.4 months (range $0.2-6$ months). 2334 of the 2546 patients (91.7\%) had positive $\mathrm{HC} 2$ hrHPV results, while 212 patients ( $8.3 \%$ ) had negative HC2 test results. Among 1135 patients with prior Pap test and HPV testing results, $7.1 \%$ patients had negative HPV test, while $8.0 \%$ had negative Pap test $(\mathrm{p}=0.38)$. Only $21(1.9 \%)$ patients had double negative results.

Table 1. Prior Pap and hrHPV test results in 1135 patients

\begin{tabular}{|c|c|c|c|c|c|c|}
\hline \multirow{2}{*}{ Category } & \multicolumn{2}{|c|}{ HPV Positive } & \multicolumn{2}{|c|}{ HPV Negative } & \multicolumn{2}{|c|}{ Total } \\
\cline { 2 - 7 } & Number & $\%$ & Number & $\%$ & Number & $\%$ \\
\hline HSIL & $442^{*}$ & 41.9 & 20 & 25.0 & $462^{*}$ & 40.7 \\
\hline LSIL & 216 & 20.5 & 8 & 10.0 & 224 & 19.7 \\
\hline ASC-H & 153 & 14.5 & 12 & 15.0 & 165 & 14.5 \\
\hline ASC-US & 161 & 15.3 & 19 & 23.8 & 180 & 15.9 \\
\hline AGC & 13 & 1.2 & 0 & 0 & 13 & 1.1 \\
\hline Negative & 70 & 6.6 & 21 & 26.3 & 91 & 8.0 \\
\hline Total & 1055 & 100 & 80 & 100 & 1135 & 100 \\
\hline
\end{tabular}

*including 2 malignant Paps

Conclusions: This largest case study demonstrates prior results of HPV testing and Pap cytology for patient with high grade cervical squamous precursor lesions in a population of women in China who had not undergone intensive prior screening. Both hrHPV and Pap cytology had similar negative rates in this time period but double negative results were less common. These results support the value of contesting in the detection of cervical cancer precursors.

1270 Implementation of a Universal Endometrial Cancer Lynch Syndrome Screening Program: Lessons Learned

Xuemei Wu, Brittany C Thomas, Jamie N Bakkum-Gamez, Casey L Swanson, Carrie L Langstraat, Myra J Wick, Sarah E Kerr. Mayo Clinic, Rochester, MN.

Background: Universal screening of endometrial cancer (EC) detects women with Lynch syndrome (LS) that may not otherwise be considered at risk. Identifying LS can prevent future morbidity and mortality from LS related cancers in the proband and her family. Implementing screening protocols can be challenging, however, due to the need for supportive infrastructure. We describe implementation of LS screening at a tertiary care center.

Design: EC patients $<71$ years old were informed of LS screening at a pre-surgical appointment. Pathology initiated reflex testing at hysterectomy; a case list was reviewed weekly to ensure compliance. Mismatch repair immunohistochemistry (MMR) served as the initial screen. Women $<51$ years with normal MMR also underwent microsatellite instability (MSI) testing. Tumors with MLH1 loss underwent MLH1 promoter hypermethylation (MLH1hm) testing. All women were called and sent standardized letters with recommended follow-up based on test results and family history. Positive screens were defined as any MMR/MSI abnormality except MLH1 loss with MLH1hm; however, any test abnormality triggered referral to medical genetics.

Results: For 277 patients screened in the first 22 months, age ranged 29-71 years (median 61). Histologies included 236 endometrioid, 20 serous, 8 other/mixed, 7 carcinosarcoma, and 4 clear cell tumors; 1 atypical complex hyperplasia and 1 ovarian cancer were inadvertently screened. Age for 13 screen positives ranged 47 to 68 years (Table 1). Errors occurred in the algorithm as follows: MSI testing in 8 women with normal MMR $>50$ years and 8 women with abnormal MMR; MLH1 hm not reflexed in 4 tumors with MLH1 loss; and a 71 year old was screened. One woman with normal MMR was later diagnosed with LS (PMS2).

\begin{tabular}{|l|l|l|}
\hline MMR loss & Germline & Histology \\
\hline MSH6 & MSH6 & E1 \\
\hline MSH6 & MSH6 & E1 \\
\hline MSH6 & MSH6 & E** \\
\hline PMS2 & MLH1 & E1 \\
\hline MSH2/6 & Neg & E2 \\
\hline MSH2/6 & Neg & E1 \\
\hline MLH1/PMS2 & Neg & E1 \\
\hline MSH6 & n/a & E1 \\
\hline MSH6 & $\mathrm{n} / \mathrm{a}$ & CS \\
\hline MSH6* & $\mathrm{n} / \mathrm{a}$ & E1 \\
\hline MSH2/6 & $\mathrm{n} / \mathrm{a}$ & E1 \\
\hline PMS2 & $\mathrm{n} / \mathrm{a}$ & E2 \\
\hline PMS2 & $\mathrm{n} / \mathrm{a}$ & E3 \\
\hline *heterogeneous loss. E $=$ endometrioid grade $1 / 2 / 3 . * *$ dedifferentiated. CS $=$ carcinosarcoma. \\
\hline
\end{tabular}


Conclusions: Screening resulted in 4 new LS diagnoses, predominantly MSH6 carriers. MSH6 carriers are overrepresented in similar EC studies, likely due to lower penetrance/later onset of disease. Three positive screens were germline negative, and 6 have not completed follow-up. The screen positive rate $(5 \%)$ is lower than other universal screening studies, possibly due to exclusion of women $>70$. Algorithm errors were infrequent, but emphasize the need for quality improvement when implementing a screening program.

1271 Mutational Analysis of BRAF and KRAS in Ovarian Serous Borderline Tumors (SBTs) and Their Subsequent Serous Carcinomas Deyin Xing, Felix Zeppernick, Yohan Suryo Rahmanto, Charlotte G Hannibal, Susanne K Kjaer, Robert Kurman, Tian-Li Wang, Ie-Ming Shih, Russell Vang. Johns Hopkins Medical Institute, Baltimore, MD; Danish Cancer Society Research Center, Copenhagen, Denmark.

Background: It is unclear whether invasive low-grade serous carcinomas (LGSCs) developing after the diagnosis of an SBT represent direct progression in all cases or if a subset of the carcinomas arise independently from peritoneal endosalpingiosis. The aim of this study was to assess the relationship between SBTs and their subsequent serous carcinomas.

Design: SBTs and their paired subsequent invasive serous carcinomas from the Danish Cancer Society-Johns Hopkins Hospital population-based study were analyzed for mutational hotspots in $K R A S$ at exon 2, codons 12-13, and $B R A F$ at exon 15, codon 600 . Results: Of 1,042 patients with SBTs, 43 patients (4\%) [34 atypical proliferative serous tumor (APST) and 9 non-invasive LGSC (niLGSC)] developed subsequent invasive serous carcinoma (40 LGSC, 3 high-grade serous carcinoma [HGSC]) with a median interval of 10 years from diagnosis of SBT to subsequent carcinoma. Of these 43 cases, 32 had available blocks with informative molecular results (SBT: 26 APST, 6 niLGSC; subsequent carcinoma: 31 LGSC, 1 HGSC). 3/32 (9\%) and 13/32 (41\%) had concordant $B R A F$ or KRAS mutations, respectively, in the paired SBTs and subsequent carcinomas. In $12 / 32(38 \%), B R A F$ and $K R A S$ were wild-type in the paired SBTs and subsequent carcinomas. $4 / 32(13 \%)$ had discordant results for $B R A F$ or KRAS between the paired SBTs and subsequent carcinomas: case 1- BRAF in APST/KRAS in subsequent HGSC; cases 2 and 3- wild-type in APST/KRAS in subsequent LGSC; and case 4- KRAS in APST/wild-type in subsequent LGSC.

Conclusions: Our molecular genetic analysis suggests that a substantial proportion of serous carcinomas which develop subsequent to the primary SBT diagnosis most likely represents direct progression from SBTs. However, that a number of cases have wild-type $B R A F / K R A S$ in SBTs and their subsequent serous carcinomas is non-informative with respect to relationship between both tumors. Our findings also confirm that in at least a small subset of cases, the subsequent carcinomas likely arise independent of the prior SBT. Further molecular studies in addition to mutational analysis of $B R A F / K R A S$ will be necessary to refine the clonal relationship between SBTs and their subsequent serous carcinomas. DX and FZ are co-first authors; I-MS and RV are co-corresponding authors.

1272 Aberrant PAX8 Expression in Well Differentiated Papillary Mesothelioma and Malignant Mesothelioma

Deyin Xing, Natalie Banet, Peter Illei. Johns Hopkins Medical Institute, Baltimore, MD. Background: Pax8 is a useful immunohistochemical marker for the diagnosis of gynecologic tract malignancies. Ovarian serous neoplasms can have morphologic overlap with both well differentiated papillary mesotheliomas (WDPM) and malignant mesotheliomas (MM). Distinguishing between peritoneal mesotheliomas and serous neoplasms involving the peritoneum is of importance in clinical management of these diseases. Pax8 is a relatively sensitive and specific marker for differentiating peritoneal epithelioid/malignant mesotheliomas and serous carcinomas, but the diagnostic value of Pax8 in WDPM remains largely unknown.

Design: Pax8 expression was assessed using a rabbit polyclonal antibody in a range of mesothelial lesions, including WDPM $(\mathrm{n}=32)$, peritoneal MM $(\mathrm{n}=34)$, pleural MM $(\mathrm{n}=48)$, adenomatoid tumors (AT, $\mathrm{n}=11)$, peritoneal inclusion cysts (PIC, $\mathrm{n}=5)$, and benign/reactive mesothelium $(n=51)$. Immunohistochemical staining for Calretinin and BerEp4 or MOC31 were also performed in most cases.

Results: The patients with peritoneal WDPM ranged in age from 8 to 77 years (mean, 50; median, 53) which were not statistically different from those with peritoneal MM (range from 30 to 82 years; mean, 56; median, 51). Pax 8 nuclear positivity was demonstrated in 20 of $32(63 \%)$ WDPM with 17 cases showing strong and diffuse nuclear staining and 3 cases patchy/focal staining. The 27 WDPM cases assessed for calretinin expression were all positive. The immunostains for BEREP4 or MOC31 were performed in 28 WDPM cases and showed that 26 cases were negative and 2 cases were weak/patchy positive. In contrast, only 4 of 34 (12\%) peritoneal MM had positive Pax8 staining (3 diffuse and 1 patchy positivity). All AT and PIC were negative for Pax8. Of the 48 pleural MM cases 2 (4\%) showed focal weak to moderate nuclear labeling for Pax8 and 2 of 51 (4\%) cases with reactive mesothelium demonstrated focal and scattered Pax8 staining. Conclusions: Pax8 is a useful marker for distinguishing MM and AT from gynecologic malignancies, but is not reliable for distinguishing WDPM from gynecologic tract lesions, especially WDPM with sold nests and more complex architecture from atypical proliferative serous (borderline) tumors and low grade serous carcinoma that can display overlapping morphologic features with WDPM. The mechanism of Pax8 expression in WDPM is not known.
1273 Squamous Morules: Re-Examination of a Well-Known Entity An Ultrastructural and Immunohistochemistry Study

Jinjun Xiong, Yihong Wang, Margaret M Steinhoff, C James Sung, M Ruhul Quddus, Grant A Jolly, W Dwayne Lawrence, Katrine Hansen. Women \& Infants Hospital, Providence, RI; Rhode Island Hospital, Providence, RI; Brown University Alpert Medical School, Providence, RI.

Background: Squamous morules (SM) are a common component of premalignant and malignant glandular lesions of the endometrium.

They are commonly associated with glandular lesions rather than squamous lesions because they arise within a specific hormonal microenvironment. They are almost always positive for $\mathrm{CD} 10$, a marker of mesonephric remnants and their related tumors. They also consistently express B-catenin, CDX2 and rP16, possibly secondary to a B-catenin gene mutation leading to overexpression of $\mathrm{CDX} 2$ and an altered $\mathrm{p} 16$ pathway. Studies show that they are neoplastic, but their nature emains unclear. For this study we used comprehensive immunohistochemistry (IHC) and electron microscopy (EM) to explore the nature of SMs.

Design: Atotal of 15 SMs were retrieved from the archival files. There were 5 cases of atypical polypoid adenomyoma, 5 cases of endometrial atypical hyperplasia, and 5 cases of endometrioid carcinoma. Study cases were stained with B-catenin, CDX2, p16, CD10, p63 and AE1/AE3. Two endometrioid carcinoma cases were processed for EM study. Results: All SMs are diffusely positive for B-catenin (nuclear and membrane stain), $\mathrm{CDX} 2, \mathrm{p} 16, \mathrm{CD} 10$ and AE1/AE3, 5 of 15 are focally positive for p63. Endometrial glands with focal positivity for CDX2 and B-catenin are associated with periglandular and intraluminal SMs. Some SM cells showed epithelial differentiation with cell-cell junctions by EM study. A few cells demonstrated squamous differentiation with the presence of desmosomes and sparse tonofilaments. Some cells remain undifferentiated. Conclusions: SMs are neoplastic as demonstrated by diffuse positivity for B-catenin a part of the Wnt pathway which plays a critical role in carcinogenesis and epithelialto-mesenchymal transition. EM and IHC studies show that SMs are epithelioid in nature with focal squamous differentiation. In addition, diffuse B-catenin and CD10 positivity suggests mesonephric or stromal differentiation. These results suggest that SMs might be a product of pluripotent stem cells. Further study of SMs to understand their nature appears warranted.

1274 Bilateral High Grade Serous Carcinoma of Ovary: A Targeted Next Generation Sequencing Study

Esther C Yoon, Jian Zhuge, Liying Han, John T Fallon, Minghao Zhong. New York Medical College at Westchester Medical Center, Valhalla, NY.

Background: Molecular events of high grade serous carcinoma of ovary (HGS-OvCa) has been characterized by using The Cancer Genome Atlas project (TCGA) by having TP 53 mutation. However, there is no comprehensive genomic or molecular profiling comparing between bilateral synchronous ovarian cancers in one patient. As HGS-OvCa can be bilateral in approximately $70 \%$ of cases, it is difficult to determine whether it's due to metastasis or independent neoplasms. In this study, we analyzed the molecular profiles of left and right $\mathrm{HGS}-\mathrm{OvCa}$ in one patient independently and compared them to TCGA data of HGS-OvCa from general population.

Design: Four patients with synchronous bilateral HGS-OvCa were selected and the H\&E slides of the cases were reviewed by expert pathologists. The tumors of left and right were of the similar size and degree of tumor involvement and morphology. First, the tumors from left and right were sequenced separately by using targeted gene sequencing (Illumina Hiseq2000). Then, they were analyzed and compared to each other. Second, the overall molecular profiles of all the samples from four patients were compared to TCGA data from 489 patients with unilateral tumor.

Results: 1) Three out of four patients shared mutations ( $>50 \%$ ) between their left and right tumors. Interestingly one case did not have any shared mutation between left and right tumor. Some of the common shared mutations between left and right tumors were: APC, CDH1, KRAS, PIK3CA and TP 53. 2) Comparing all eight tumors with TCGA data, most frequently involved mutations were TP53 gene, KRAS and FGFR2 followed by APC, PIK3CA, PTEN and FLT3. No pathologic BRCA1 and BRCA2 germline mutations were seen in any of the cases. The analysis of bilateral tumor showed similar high frequency of TP53 mutations as seen in TCGA data using unilateral tumor. Furthermore, bilateral tumors showed different frequency of other mutations. Conclusions: In this study, we established high level of tumor heterogeneity in synchronous carcinomas of ovary which is distinct from previous TCGA data. Also, we were able to identify molecular profiles of each left and right HGS-OvCa in each patient and demonstrated there are significant overlaps.

1275 Characterization of the Surface Expression of PD-1/PD-L1 Signaling Proteins in High-Grade Serous Ovarian Carcinoma

Ju-Yoon Yoon, Jocelyn M Stewart, Christopher Go, Marcus Bernardini, Blaise Clarke, Patricia Shaw, Benjamin Neel, Laurie Ailles. University of Toronto, Toronto, ON, Canada; University Health Network, Toronto, ON, Canada; NYU-Langone Medical Center, Bronxville, NY.

Background: The immune checkpoint is poorly characterized in high-grade serous ovarian carcinoma (HGSOC), with only modest response rates with inhibitors observed. However, response can be durable in select patients, and the identification of these patients remains an elusive challenge.

Design: Rapid analysis of 363 cell surface antigens using a high-throughput flow cytometry platform was performed on eight specimens of HGSOC, with each sample being co-stained with anti-CD45 antibody. mRNA levels from The Cancer Genome Atlas (TCGA) dataset were also examined.

Results: PD-1 and PD-L1/2 levels were low in both CD45(-)/(+) populations in HGSOC, with higher expression of CTLA-4. CD45(+) population exhibited higher expression of 
CD28, CD80 and CD86 (vs. CD45(-)). Expression levels of these proteins positively correlated with one another within the respective $\mathrm{CD} 45(-) /(+)$ populations, but not between the two populations. In the CD45(-) population, TWEAK levels correlated positively with $6 / 7$ immune checkpoint proteins. Other members of the TNF super family (TNFSF), including TNF, Fas ligand and TRAIL, showed a similar trend. The mRNA levels for both the TNFSF and immune checkpoint genes positively correlated with 11/13 of NF-kappa-B-related gene mRNA levels. When we examined the genes used to stratify HGSOC into four molecular subtypes, $3 / 3$ of the genes marking the immunoreactive subtype showed the identical relationship with the TNFSF, NF-kappa-B and immune checkpoint gene mRNA levels. All three mRNA sets correlated negatively with the number of copy number alterations.

Conclusions: Our data suggests that the immune checkpoint proteins are coregulated with one another within the CD45(-)/(+) populations, but not between the two populations. Within the CD45(-) population, mostly comprised of the carcinoma cells, we hypothesize that select TNFSF members lie upstream of the immune checkpoint genes, likely by activating the NF-kappa-B pathway. This response is variable between the molecular subtypes of HGSOC, being most marked in the immunoreactive subtype. Our data also suggests that CTLA-4 may be the better therapeutic target ( $v s$. PD-1), and suggests that the benefits of immune checkpoint inhibition may be limited to a select HGSOC patients that can be defined by cell surface marker expression.

\section{Androgen Receptor Expression in Endometrial Carcinoma}

Sara Zadeh, Linda Duska, Kristen Atkins, Anne Mills. The University of Virginia, Charlottesville, VA.

Background: Endometrial carcinoma (ECA) is frequently hormonally-driven and can be treated with hormonal antagonists, yet hormone receptor status is not routinely assessed. In particular, little is known about the significance of androgen receptor (AR) in ECA. Androgen has anti-proliferative effects in the endometrium and could serve a similar role to progesterone in curbing estrogen-dependent neoplasia. This is supported by work showing that loss of AR correlates with poor survival in ECA. Furthermore, AR immunohistochemistry (IHC) has been suggested as a supplement to progesterone receptor (PR) IHC when evaluating patients for progesterone-based therapy, since AR can bind to progesterone; however few studies analyze the relationship between AR and PR in ECA. Finally, it is unknown whether a subset of ECA expresses AR without concomitant ER: a group that might benefit from androgen antagonistic therapy. We herein investigate AR expression across ECA subtypes and compare expression to $\mathrm{ER} / \mathrm{PR}$.

Design: AR, ER, and PR IHC was performed on an endometrial tissue microarray containing 50 ECA in a variety of morphologic subtypes as well as 20 benign and 10 hyperplastic endometria. Scoring was based on the Liverpool endometrial steroid quick score with positive staining classified as low, moderate, or high.

Results: AR was expressed by $54 \%(27 / 50)$ of ECA [ $60 \%$ low grade endometrioid carcinomas (LGEC), $70 \%$ high grade endometrioid carcinomas (HGEC), $70 \%$ serous carcinomas (SC), $50 \%$ malignant mixed mullerian tumors (MMMT), and $20 \%$ clear cell carcinomas (CCC)]. Average patient age for AR+ tumors was 71 years vs. 68 for AR- tumors. Most AR+ tumors had low (52\%) to moderate $(26 \%)$ expression. High AR expression was restricted to $50 \%$ of SC. AR expression most often occurred with ER staining, although $1 \mathrm{SC}$ and 3 MMMT showed low-moderate AR expression in the absence of ER. AR + cancers usually co-expressed PR although $20 \% \mathrm{HGEC}, 20 \% \mathrm{SC}$, $10 \%$ CCC, and 30\% MMMT showed AR without PR. Negative AR was seen more often with recurrence ( $44 \%$ vs $33 \%$ ) however this trend was not statistically significant. Conclusions: AR positivity is seen in over half of ECA, including the majority of LGEC, HGEC, and SC. Only high-grade cases showed AR in the absence of PR, failing to support a role for AR in the selection of cases for progestin therapy. However, a small subset of high-grade cases showed AR-positivity without concomitant ER, suggesting a possible role for androgen inhibition in these difficult to treat tumors.

1277 Diagnostic Challenges in Mixed Endometrial Carcinomas: Clinical Relevance of Different Neoplastic Components

Gian Franco Zannoni, Tommaso Bizzarro, Frediano Inzani, Damiano Arciuolo, Giovanni Scambia, Esther Rossi. Catholic University, Rome, Italy.

Background: Mixed endometrial carcinomas (MEC) refer to tumors characterized by 2 or more distinct histotypes mostly comprised of endometrioid (EC) and serous papillary/ clear cell carcinomas (SC/CC). The specific quantification of these distinct components represents a challenging and critical point for both prognosis and management. Herein, we analyze a large series of MEC and compare them with $\mathrm{EC}$ and $\mathrm{SC} / \mathrm{CC}$.

Design: We evaluated a series of 69 MEC betweenJanuary 2002 and December 2015 . We compared the MEC series with 186 EC (including 117 endometrioid G3), 31 SC and $38 \mathrm{CC}$. The prognostic implication of the percentage of each component was analyzed. Results: Among the $69 \mathrm{MEC}$, those patients older than $45 \mathrm{y} / \mathrm{o}$ represent the significant population with $52.2 \%$ of them with stage III-IV disease. A similar result was found among pure SC. The comparative analysis of some prognostic parameters (multifocality, vascular invasion and lymph-node metastasis) underlined that MEC with a type II component larger than $10 \%$ represent a more aggressive entity. However, relapse, disease free survival (DFS), mortality and overall survival (OS) are statistically significant $(\mathrm{p}<0.05)$ in $\mathrm{EC}-\mathrm{SC}(\mathrm{SC}<$ or $>10 \%)$, EC-CC $(\mathrm{CC}<$ or $>10 \%)$ whereas are not significant $(\mathrm{p}>0.05)$ in $\mathrm{SC}-\mathrm{CC}(\mathrm{SC} / \mathrm{CC}<$ or $>10 \%)$.

Conclusions: $\mathrm{MEC}$, including also cases with less than $10 \%$ of SC/CC, show features as aggressive as those of pure $\mathrm{SC} / \mathrm{CC}$. In this perspective, MEC should be followed by personalized and tailored managements. The presence of different components suggests different pathogenic and metastatic processes when compared with pure carcinomas.
1278 Ovarian Serous Carcinomas with Mixed Features of High Grade and Low Grade Serous Carcinoma Display Heterogeneous Genome Wide Copy Number Variation Profiles

Shabnam Zarei, Chen Wang, Jean-Pierre A Kocher, Yan Wang, Debra A Bell, Sarah E Kerr. Mayo Clinic, Rochester, MN.

Background: Classic high and low grade serous carcinomas (HGSC and LGSC) are thought to be distinct at a molecular level. HGSC have TP53 mutations (96\%) and widespread copy number changes, whereas LGSC have KRAS, BRAF and PTEN mutations and are near diploid. Little is known about the molecular profile when mixed morphologic features of HGSC and LGSC are seen in the same patient. We previously showed that majority of these cases ( $70 \%$ ) do not show TP53, KRAS, BRAF or PTEN mutations. In this study we describe genome wide copy number variation (CNV) in such tumors.

Design: A review of serous tumors at our institution between 1995-2012 revealed 376 pure HGSC, 19 pure LGSC and 15 HGSC apparently co-existing with LGSC. We selected this group with mixed features of LGSC and HGSC, 15 cases with pure HGSC and 19 with pure LGSC using MD Anderson diagnosis criteria. Low pass whole genome sequencing (WGS) was performed using DNA from tumor areas enriched by macrodissection from FFPE tissue sections. In mixed cases, low and high grade areas were extracted separately. A dedicated CNV bioinformatics pipeline named Wandy processed the data. Coverage profiles were segmented into broad- and focal-CNV events to facilitate low- versus high-grade classifications, using a regression-tree approach. Results: All pure LGSC cases harbor broad CNV events related to aneuploidy, with few to no focal amplifications or loss. In contrast, HGSC cases tend to have "spike-like" focal events on top of aneuploidy events. In the group with mixed features of HGSC and LGSC more heterogeneity was seen: $27 \%(4 / 15)$ showed progression from LGSC to HGSC and $73 \%$ showed either pure low grade $(33 \%, 5 / 11)$ or pure high grade $(40 \%$, $6 / 11)$ genotype in both high grade and low grade components.

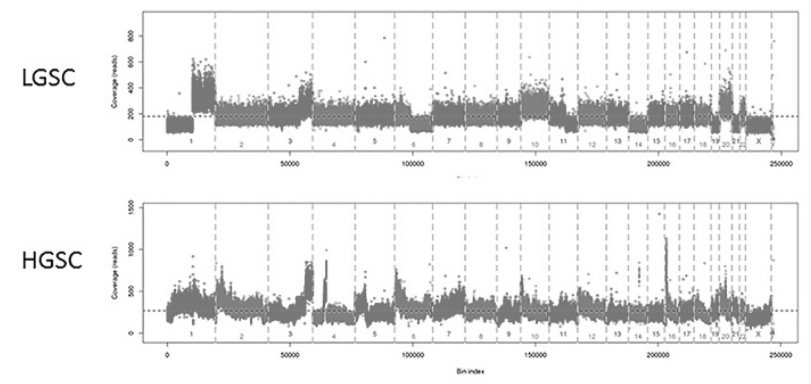

Conclusions: Low-pass WGS may be used as a tool to distinguish pure LGSC from pure HGSCs. We found that the total number of focal CNV events could be a single metric to clearly separate pure LGSC and HGSC cases. Mixed grade carcinomas showed more heterogeneity, suggesting only some of these tumors represent progressive low to high grade disease. Additional analysis is needed to determine if CNV metrics correlate with prognosis in morphologically ambiguous cases.

1279 SATB2 Is a Useful Marker in Differentiating Metastatic Poorly Differentiated Gastric Carcinomas with Signet Ring Cell Features from Appendiceal Goblet Cell Carcinoids in Ovary

Lingxin Zhang, Chen Yang, Dengfeng Cao. Pathology \& Immunology, Saint Louis, MO. Background: Primary poorly-differentiated tumors of the ovary with signet ring cell feature are exceedingly rare (in the literature only 3 cases have been reported in associated with mucinous borderline tumors), and the vast majority $(>99 \%)$ are metastatic. The two most common primary sites are the stomach (signet ring carcinoma, SRCC) and appendix (goblet cell carcinoid or carcinoma ex-goblet cell carcinoid, GCC). In the lack of adequate clinical or radiologic input, identifying unknown primary sites in these cases is a game of 'hit-or-miss,' since SRCC and GCC can resemble each other morphologically and often demonstrate overlapping immunohistochemical (IHC) profiles in cytokeratins and CDX2. Neuroendocrine markers, e.g. chromogranin and synaptophysin, are only positive in a subset cells of GCCs, with high interobserver discrepancies. In this study, we investigated the diagnostic utility of SATB2 in differentiating metastatic SRCCs and GCCs in the ovary.

Design: Tissue microarrays were constructed on SRCCs and GCCs metastatic to the ovary using $3 \mathrm{~mm}$ core tissue (3 cores per case). IHC stainings for SATB2, CDX2, chromogranin, and synaptophysin were performed. The stainings for SATB2 were semi-scored as $0(<1 \%$ cells $), 1+(1-30 \%), 2+(31-60 \%), 3+(61-90 \%), 4+(>90 \%)$ by all the authors.

Results: 7 gastric SRCCs and 15 appendiceal GCCs metastatic to the ovary were identified from the institutional archive, the primary sites definitively supported by clinicopathologic data. Diffuse nuclear staining (score 3 to 4 ) of SATB2 was present in 14 of 15 (93\%) GCCs, and absent in SRCCs (score 0 to 1), with satisfactory interobserver consistency.

\begin{tabular}{|l|c|c|c|c|c|}
\hline SATB2 Score & 0 & $1+$ & $2+$ & $3+$ & $4+$ \\
\hline Appendix & 1 & -- & -- & 2 & 12 \\
\hline Stomach & 6 & 1 & -- & -- & -- \\
\hline
\end{tabular}

In contrast, $\mathrm{CDX} 2$, chromogranin, and synaptophysin had heterogeneous staining pattern across the two entities. 


\begin{tabular}{|l|c|c|c|}
\hline IHCs & CDX2 (\%) & CGA (\%) & SYN (\%) \\
\hline Appendix & 98.7 & 4.5 & 14.3 \\
\hline Stomach & 77.1 & 30.0 & 17.2 \\
\hline
\end{tabular}

Conclusions: The diffuse nuclear staining pattern of SATB2 is consistently identified in appendiceal GCCs and absent in gastric SRCCs that are metastatic to the ovary. Compared to traditional markers, SATB2 is a novel useful marker in identifying primary anatomic sites in metastatic signet ring cell lesions in the ovary.

\section{Assessment of Clinical and Pathological Factors Contributing} to Negative LEEP

Youran Zou, Gloria Zhang, Charles V Biscotti, Jerome L Belinson, Bin Yang. Cleveland Clinic, Cleveland, $\mathrm{OH}$.

Background: Accurate diagnosis of squamous dysplasia on cervical biopsy is important not only for guiding treatment of HSIL lesions but also for preventing over treatment. We have reviewed a large cohort of LEEP cases and assessed clinical and pathological factors contributing to negative LEEP.

Design: All LEEP cases from 2007, 2009, 2013, and 2015 were identified in our pathology archive. Prior diagnosis, p16 immunostaining results, and LEEP diagnosis were recorded. Factors affecting clinical decisions including cervical cytology results, HPV testing and others were noted. LACK of HSIL (either benign or CIN1) defined as negative LEEP. Cases with glandular abnormalities have been excluded from this study. Consistent application of 16 immunostain by most of our pathologists began in 2012. To determine whether p16 immunostain affects negative LEEP rate, we compared negative LEEP rate in 2007-2009 (prior to p16 application) and in 2013-2015 (post p16 application).

Results: A total of 1,256 LEEP cases were identified, consisting of 674 cases prior to $\mathrm{p} 16$ and 582 cases post p16 application. Negative LEEP rate was $42.7 \%(288 / 674)$ prior to $\mathrm{p} 16$ and was $36.1 \%(210 / 582)$ post $\mathrm{p} 16$ application $(\mathrm{p}=0.02)$. Among negative LEEP cases, pathologists' contribution (CIN2+ diagnosis on biopsy with subsequent negative LEEP) was $61.1 \%(176 / 288)$ prior to $\mathrm{p} 16$ and $54.8 \%(115 / 210)$ post p16 application. Clinicians' contribution (performed LEEP either with no biopsy or biopsy diagnosis as benign or CIN1) was $39.9 \%$ (112/288) prior to p16 and $46.2 \%(95 / 210)$ post p16 application.

Conclusions: Negative LEEP is contributed both by pathologists and by clinicians. Application of $\mathrm{p} 16$ has decreased the negative LEEP rate contributed by pathologists, presumably with more accurate $\mathrm{CIN} 2+$ diagnosis. Conversely clinicians' contribution to negative LEEP has relatively increased after wide application of $\mathrm{p} 16$ for HSIL lesions.

1281 Invasive Implants of Ovarian Serous Borderline Tumor: Significant Association with KRAS but Not BRAF Mutation

Tao Zuo, Serena Wong, Natalia Buza, Pei Hui. Yale University, New Haven, CT.

Background: In contrast to non-invasive implants, invasive implants of serous borderline tumor/atypical proliferative serous tumor (SBT/APST) are associated with adverse clinical outcome and currently classified as low-grade serous carcinoma by the 2014 WHO classification. Somatic mutations in $K R A S$ and $B R A F$ have been reported in more than $50 \%$ of SBT/APST. In this study, we investigated $K R A S$ and $B R A F$ mutation frequencies in the two types of SBT/APST implants in correlation with clinical outcome. Design: Thirty-seven implants of SBT/APST from 34 patients were included. The implants were reviewed and reclassified according to the 2014 WHO criteria into 18 invasive and 19 non-invasive implants. DNA was extracted from microdissected tissue samples and subjected to PCR amplification followed by single-strand conformation polymorphism analysis (SSCP) to detect the presence of KRAS exon 2 and BRAF V600E mutations. The implant type and presence of KRAS/BRAF mutations were correlated with clinical follow-up data

Results: $K R A S$ and $B R A F$ mutation analyses were informative in all but one noninvasive implant (Table 1). KRAS mutation was found in 11 of 18 invasive implants $(61 \%)$ and 4 of 18 non-invasive implants (22\%) ( $p=0.018$; Chi Square). BRAF V600E mutation was seen in 1 of 19 non-invasive implants $(5 \%)$ and none of 18 invasive implants harbored BRAF V600E mutation ( $0 \%$ ). Two patients had two implants (each one invasive and one non-invasive) analyzed and KRAS mutation was seen only in the invasive implants but not in the non-invasive implants in both patients. Invasive implants were more frequently associated with high stage disease ( $p=0.007$; Chi Square). Eleven of 19 patients $(58 \%)$ with stage IIIC disease were found to have KRAS mutation, while only one patient with stage IIB had the mutation. However, Kaplan-Meyer survival analyses did not show significant survival differences between invasive and noninvasive implants and between implants with and without $K R A S$ mutation, although a prognostic trend was suggested.

\begin{tabular}{|l|l|l|l|}
\hline & Invasive Implant $(\mathrm{N}=18)$ & Non-invasive Implant $(\mathrm{N}=19)$ & Total $(\mathrm{N}=37)$ \\
\hline KRAS Mutation & $11 / 18(61 \%)$ & $4 / 18(22 \%)$ & $15 / 36(42 \%)$ \\
\hline BRAF Mutation & $0 / 18(0 \%)$ & $1 / 19(5 \%)$ & $1 / 37(3 \%)$ \\
\hline
\end{tabular}

Conclusions: Invasive implants of SBT/APST are significantly associated with KRAS mutations and a wild-type $B R A F$. PCR-SSCP is a highly sensitive and specific method for detection of KRAS/BRAF mutations in small tissue samples such as microscopic implants of SBT/APST. Additional studies are necessary to ascertain the prognostic values of $K R A S / B R A F$ mutation analysis of implants of SBT/APST.

\section{Head and Neck Pathology}

1282 SMARCB1 (INI-1)-Deficient Sinonasal Carcinoma: A Series of 33 Cases Expanding the Morphological and Clinicopathological Spectrum of a Recently Described Entity

Abbas Agaimy, Arndt Hartmann, Cristina R Antonescu, Simon Chiosea, Samir K ElMofty, James Lewis, Stacey E Mills, Ann Sandison, Roderick HW Simpson, William H Westra, Justin A Bishop. University Hospital of Erlangen, Erlangen, Germany; Memorial Sloan Kettering Cancer Center, New York, NY; University of Pittsburgh Medical Center, Pittsburgh, PA.

Background: SMARCB1-deficient sinonasal carcinoma is a recently described sinonasal tumor entity characterized by loss of nuclear SMARCB1 (INI1).

Design: To more fully characterize the clinicopathological spectrum of this proposed entity, we analyzed 33 cases collected from multiple medical centers.

Results: Both sexes were affected equally (age range: 19 to 87 yrs; median, 52). All patients presented with locally advanced disease $(T 4, n=27)$ involving the sinuses and variably the nasal cavity. Twenty-five patients received surgery and/or radiochemotherapy with curative intent. At last follow-up, $58 \%$ of patients died of disease ( 0 to 102 months; median, 16), 2 were alive with disease, and 1 died of unrelated cause. Only 6 patients (29\%) were alive without disease (11-115 months; median, 34). Original diagnoses of archival cases were most often sinonasal undifferentiated carcinoma $(\mathrm{n}=12)$ and non-keratinizing/basaloid squamous cell carcinoma $(\mathrm{n}=4)$. Histologically, most tumors displayed either predominantly basaloid $(55 \%)$ or plasmacytoid/rhabdoid (38\%) morphology. The plasmacytoid/rhabdoid form consisted of sheets of tumor cells with eccentric nuclei and eosinophilic cytoplasm, while similar cells were rare and singly distributed in the basaloid variant. Glandular differentiation was seen in a few tumors. Squamous differentiation and surface dysplasia were absent. By immunohistochemistry (IHC), the tumors were positive for pankeratin (97\%), CK5 $(65 \%)$, p63 (50\%) and CK7 (43\%). NUT IHC was negative. Epstein-Barr virus and high risk human papillomavirus were not detected by in situ hybridization. Loss of SMARCB1 (INI1) expression was confirmed for all 33 tumors by IHC. SMARCB1 deletions were detected in 19/23 cases by FISH.

Conclusions: SMARCB1-deficient sinonasal carcinoma represents a distinctive emerging poorly/undifferentiated sinonasal carcinoma that 1) cannot be better classified as another specific tumor type, 2) has consistent (albeit variable) histopathological findings, and 3) demonstrates an aggressive clinical course. This diagnosis should be considered in any difficult-to-classify sinonasal carcinoma, in order to optimize therapy and to further delineate this probably underdiagnosed disease.

1283 Extranodal Lymphoid Neoplasms of the Head and Neck: A Retrospective Analysis

Yahya Al-Ghamdi, Faizan Malik, Smita Patel, Brett Mahon, Paolo Gattuso. Rush University Medical Center, Chicago, IL.

Background: Lymphoid neoplasms of head and neck (HN) account for $12-15 \%$ of all head and neck tumors and $5 \%$ of all malignancies. This study aimed to investigate the clinicopathological features of lymphoid neoplasms arising in mucosal sites of HN. Additionally, considering its therapeutic target and its association with a more favorable outcome in diffuse large B-cell lymphomas (DLBCL), CD30 immunoexpression was assessed.

Design: We searched the archives of our department from 2000 to 2016 for lymphoid neoplasms diagnosed at HN sites. The cases were divided to two groups: Primary localized without bone marrow involvement; Secondary - diagnosed in the HN with bone marrow involvement or relapsed cases of a known lymphoma. Clinical characteristics, autoimmune association, histologic subtype, and CD30 immunoexpression were analyzed.

Results: Sixty cases were retrieved, including 31 women and 29 men. The mean age was 59.78 years (range: 16-91). Among 49 cases whose medical charts were available, 35 were primary and 14 were secondary. The sites of involvement include tonsils $(\mathrm{n}=25 ; 41.6 \%)$, nasopharynx $(\mathrm{n}=12 ; 20 \%)$, base of the tongue $(\mathrm{n}=10,16.7 \%)$, larynx $(\mathrm{n}=7 ; 11.7 \%)$, maxillary sinuses $(\mathrm{n}=3 ; 5 \%)$, and oral mucosa $(\mathrm{n}=3 ; 5 \%)$. Histologic subtypes include DLBCL $(\mathrm{n}=22 ; 36.7 \%)$, mantle cell lymphoma $(\mathrm{n}=8$; $13.3 \%)$, marginal zone lymphoma $(\mathrm{n}=7 ; 11.7 \%)$, peripheral T-cell lymphoma $(\mathrm{n}=6$; $10 \%)$, follicular lymphoma $(\mathrm{n}=4 ; 6.7 \%)$, extranodal NK/T cell lymphoma $(\mathrm{n}=4 ; 6.7 \%)$, small lymphocytic lymphoma/chronic lymphocytic leukemia $(\mathrm{n}=3,5 \%)$, unclassifiable B-cell lymphoma, intermediate between DLBCL and Burkitt lymphoma $(\mathrm{n}=2 ; 3.3 \%)$. In addition, there were single cases of plasmablastic lymphoma, Burkitt lymphoma, and ALK-negative anaplastic large cell lymphoma. Among 16 DLBCL cases assessed for CD30 immunoexpression, 3 were positive. Two DLBCL cases were EBV positive by in-situ hybridization. Eleven patients had an autoimmune association, including hypothyroidism $(n=4)$, rheumatoid arthritis $(n=3)$, inflammatory bowel disease $(n=2)$, Sjögren's syndrome $(n=1)$, and autoimmune hepatitis $(n=1)$. Five of these 11 patients had a primary lymphoma. An unclassified T-cell lymphoma developed in a patient with combined variant immunodeficiency. Two cases were associated with human immunodeficiency virus infection.

Conclusions: Both B- and T-cell lymphoid neoplasms of head and neck are not uncommon and should always be considered in the differential diagnosis. Most cases in our study were primary lymphomas. The most common site of involvement was the Waldeyer ring. Our findings suggest an immune dysregulation association. 